\title{
MODELO PARA DIMENSIONAMENTO ECONÔMICO DE SISTEMAS DE RECALQUE EM PROJETOS HIDROAGRÍCOLAS
}

\section{JoÃo LUIS ZOCOLER}

Engenheiro Agrônomo

Orientador: Prof. Dr. JOSÉ ANTÔNIO FRIZZONE

Tese apresentada à Escola Superior de Agricultura

"Luiz de Queiroz", Universidade de São Paulo, para obtenção do título de Doutor em Agronomia, Área de Concentração: Irrigação e Drenagem.

\section{PIRACICABA}

Estado de São Paulo - Brasil

Fevereiro - 1998 
Dados Internacionais de Catalogação na Publicaçāo (CIP) DIVISĀo DE BIBLIOTECA E DOCUMENTAÇĀo - Campus "Luiz de Queirož"/USP

Zocoler, João Luis

Modelo para dimensionamento econômico de sistemas de recalque em projetos hidroagrícolas / João Luis Zocoler, 1998.

$107 \mathrm{p}$.

Tese (doutorado) - Escola Superior de Agricultura Luiz de Queiroz, 1998. Bibliografia.

1. Análise agricola 2. Hidráulica agrícola 3. Modelo matemático 4. Sistema de recalque I. Título

CDD 621.67

631.7 


\section{MODELO PARA DIMENSIONAMENTO ECONÔMICO DE SISTEMAS DE RECALQUE EM PROJETOS HIDROAGRÍCOLAS}

JOÃo LUIS ZOCOLER

Aprovada em: 31.03 .1998

Comissão julgadora:

Prof. Dr. José Antônio Frizzone ESALQ / USP

Prof. Dr. Rubens Duarte Coelho ESALQ / USP

Prof. Dr. Tarlei Arriel Botrel ESALQ / USP

Prof. Dr. Rodrigo de Mello Porto $\mathrm{EESC} / \mathrm{USP}$

Prof. Dr. João Carlos Cury Saad FCAV / UNESP

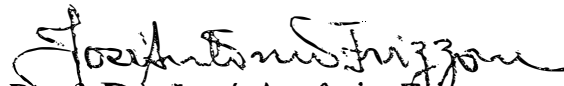
Proff Dr. José Antônio FHzzone Orientador 
Aos meus pais, João e Gentila,

pelos esforços, compreensão e apoio

na formação moral e intelectual dos seus filhos,

\section{MINHA HOMENAGEM}

À minha esposa Jeniana, que conduziu

com afinco a educação dos nossos

filhos quando estive ausente;

Ao meu filho João Gustavo, que sempre

se comportou com responsabilidade

e dedicação nos afazeres;

E ao meu filho Lucas, que com suas

travessuras muitas vezes me aliviou

das tensões do trabalho;

OFEREÇO E DEDICO 


\section{AGRADECIMENTOS}

À Deus, criador intrínseco do universo - fonte inesgotável da ciência - e tudo quanto a razão não pode explicar ou compreender.

Ao PICDT-CAPES, pela concessão da bolsa de estudo.

Ao Departamento de Ciência do Solo e Engenharia Rural da Faculdade de Engenharia de Ilha Solteira, pela liberação para cursar o doutorado.

Ao Departamento de Engenharia Rural da Escola Superior de Agricultura 'Luiz de Queiroz", pelo acolhimento e oportunidade concedida para realização deste curso.

Ao Prof. José Antônio Frizzone, DER-ESALQ-USP, pelo apoio no curso e pela orientação no trabalho de tese e outros mais.

Ao Prof. Rubens Duarte Coelho, DER-ESALQ-USP, pelo incentivo e sugestões dadas no trabalho de tese, bem como pela revisão do "Summary".

Ao Prof. Rodrigo de Mello Porto, DHS-EESC-USP, pela orientação na Revisão de Literatura.

Ao Prof. Fernando Brás Tangerino Hernandez, pelo fornecimento dos dados técnicos do equipamento pivô-central da Fazenda Santa Clarisse.

Aos professores e colegas do Curso de Pós-Graduação em Irrigação e Drenagem, que formal ou informalmente contribuiram em minha formação científica e moral.

Aos funcionários do DER-ESALQ-USP, pelo empenho em atender bem os alunos da pós-graduação.

Aos funcionários da Biblioteca Central da ESALQ, particularmente do Setor de Referência e Empréstimo-Entre-Bibliotecas/Comutação Bibliográfica, pelo excelente atendimento e serviços prestados. 


\section{SUMÁRIO}

Página

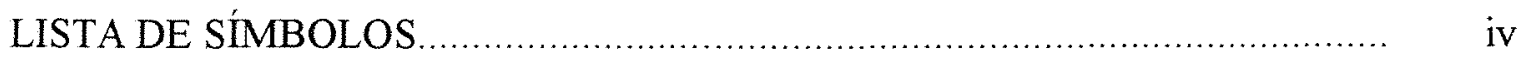

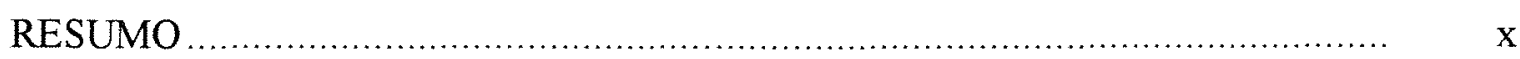

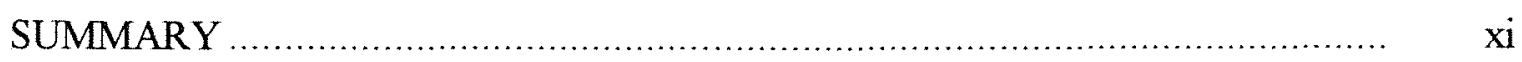

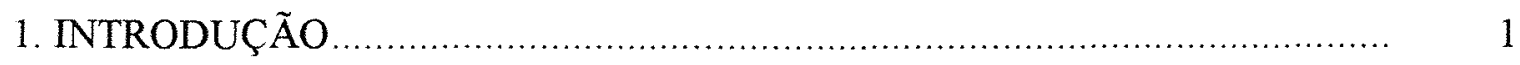

2. REVISÃO DE LITERATURA …...........................................................

2.1. Parâmetros econômicos para avaliação de uma instalação elevatória ................ 3

2.1.1. Depreciação e remuneração do capital ..................................................... 3

2.1.2. Reparos e manutenção .................................................................. 5

2.1.3. Custos com energia para o bombeamento …........................................... 7

2.2. Recursos energéticos para acionamento dos sistemas de recalque e análise comparativa de custos de sistemas a óleo diesel e à eletricidade............. 11

2.3. Diâmetro econômico para uma instalação elevatória ....................................... 19

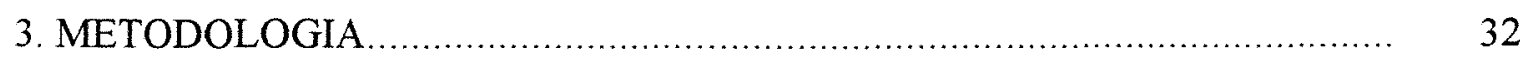

3.1. Custo fixo anual total do sistema ........................................................ 33

3.1.1. Metodologia para estimativa do custo do equipamento novo ...................... 33

3.1.2. Custo dos componentes hidráulicos ................................................... 34

3.1.3. Custo da linha e equipamentos elétricos.................................................. 36

3.1.4. Custo do motor à combustão e acessórios ……........................................ 38

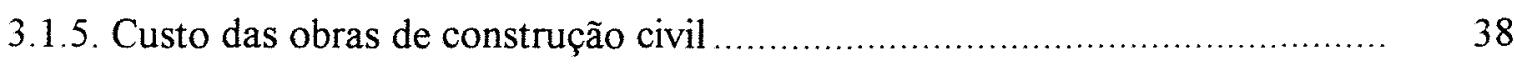

3.2. Custo variável anual do sistema ................................................................. 39

3.2.1. Custo anual de bombeamento para bombas acionadas por motor à combustão ...................................................................... 40

3.2.2. Custo anual de bombeamento para bombas acionadas

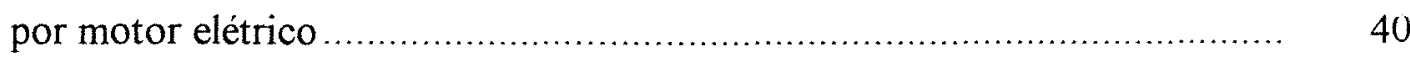

3.2.2.1. Modalidades de aplicação das tarifas de energia elétrica........................... 41 


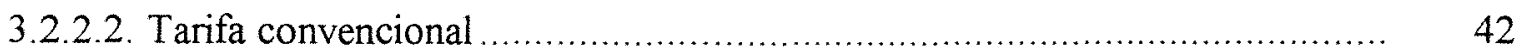

3.2.2.2.1. Faturamento da demanda...................................................... 42

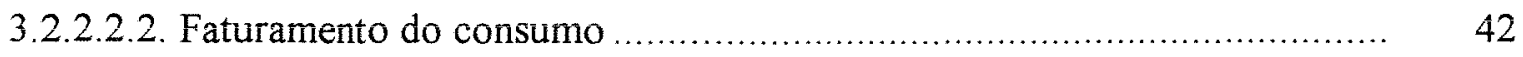

3.2.2.2.3. Ajuste do fator de potência .............................................. 43

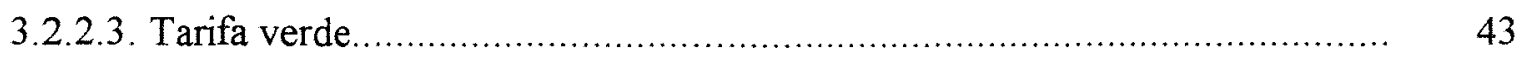

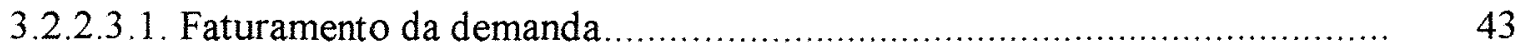

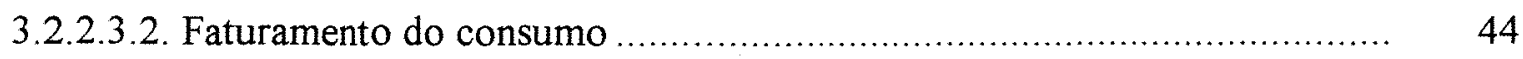

3.2.2.3.3. Ajuste do fator de potência ............................................... 45

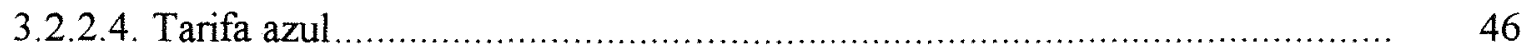

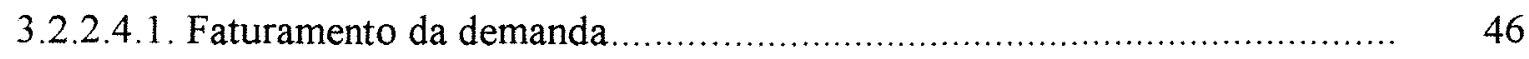

3.2.2.4.2. Faturamento do consumo ..................................................... 48

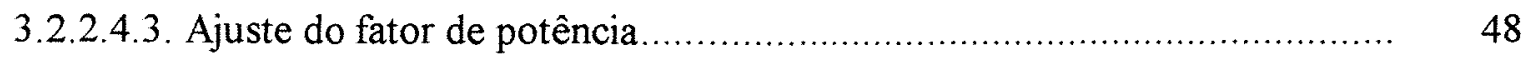

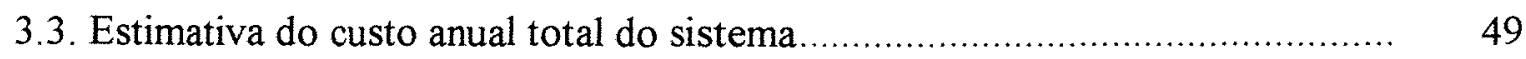

3.3.1. Sistema com bomba hidráulica acionada por motor à combustão ................ 49

3.3.2. Sistema com bomba hidráulica acionada por motor elétrico na tarifa convencional sem o beneficio da Portaria 105 do DNAEE....................... 50

3.3.3. Sistema com bomba hidráulica acionada por motor elétrico na tarifa convencional com o beneficio da Portaria 105 do DNAEE ........................ 51

3.3.4. Sistema com bomba hidráulica acionada por motor elétrico na tarifa verde sem o beneficio da Portaria 105 do DNAEE ................................. 51

3.3.5. Sistema com bomba hidráulica acionada por motor elétrico na tarifa verde com o beneficio da Portaria 105 do DNAEE................................ 52

3.3.6. Sistema com bomba hidráulica acionada por motor elétrico na tarifa azul sem o beneficio da Portaria 105 do DNAEE ................................ 53

3.3.7. Sistema com bomba hidráulica acionada por motor elétrico na tarifa azul com o beneficio da Portaria 105 do DNAEE ................................ 54

3.4. Modelo para cálculo do diâmetro econômico da tubulação de recalque ........... 55

3.4.1. Sistema com bomba hidráulica acionada por motor à combustão ............... 56

3.4.2. Sistema com bomba hidráulica acionada por motor elétrico na tarifa convencional sem o beneficio da Portaria 105 do DNAEE. 
3.4.3. Sistema com bomba hidráulica acionada por motor elétrico na tarifa convencional com o beneficio da Portaria 105 do DNAEE

3.4.4. Sistema com bomba hidráulica acionada por motor elétrico na tarifa verde sem o beneficio da Portaria 105 do DNAEE

3.4.5. Sistema com bomba hidráulica acionada por motor elétrico na tarifa verde com o beneficio da Portaria 105 do DNAEE

3.4.6. Sistema com bomba hidráulica acionada por motor elétrico na tarifa azul sem o beneficio da Portaria 105 do DNAEE

3.4.7. Sistema com bomba hidráulica acionada por motor elétrico na tarifa azul com o beneficio da Portaria 105 do DNAEE

4. APLICAÇÃO E DISCUSSÃO.

4.1. Diâmetro da tubulação de recalque que minimiza o custo anual total do sistema de recalque

4.2. Custos do sistema de recalque com bomba hidráulica acionada por motor à combustão e motor à eletricidade nas diversas modalidades de tarifação

4.3. Sensibilidade dos custos do sistema com bomba hidráulica acionada por motor à eletricidade em relação ao comprimento da linha de alta tensão

5. CONCLUSÕES

REFERÊNCIAS BIBLIOGRÁFICAS.

APÊNDICE 1

APÊNDICE 2

APÊNDICE 3 88

APÊNDICE 4 90

APÊNDICE 5 92

APÊNDICE 6 94

APÊNDICE 7 96

APÊNDICE 8 98

APÊNDICE 9. 100

APÊNDICE 10 102

APÊNDICE 11 


\section{LISTA DE SÍMBOLOS}

$\phi$ - diâmetro da tubulação de recalque (m)

$\gamma$ - peso especifico da água, assumido como $9806 \mathrm{~N} / \mathrm{m}^{3}$

$\eta_{\mathrm{BH}}$ - rendimento da bomba hidráulica

$\eta_{M E}$ - rendimento do motor elétrico

ACB - área da casa de bombas $\left(\mathrm{m}^{2}\right)$

$a_{i}$ e $b_{i}$ - coeficientes de ajuste da regressão multiplicativa de custo do i-ésimo equipamento em função de seu diâmetro ou potência nominal

$\mathrm{AJ}$ - ajuste anual do fator de potência $(\$)$

CABO - custo anual de bombeamento $(\$)$

CAE - custo dos acessórios elétricos (\$)

CAMR - custo anual com manutenção e reparos (\$)

CATS - custo anual total do sistema (\$)

$\mathrm{CBC}$ - custo do banco de capacitores (\$)

$\mathrm{CBH}$ - custo da bomba hidráulica (\$)

CCB - custo da casa de bombas (\$)

CCC - custo da construção civil para obras de acabamento médio $\left(\$ / \mathrm{m}^{2}\right)$

$\mathrm{CCH}$ - custo dos componentes hidráulicos $(\$)$

CCP - custo do comando de partida (\$)

$\mathrm{CC}_{\mathrm{u}}$ - consumo médio de combustível do motor por unidade de potência útil ( $\mathrm{m}^{3} / \mathrm{W} . \mathrm{h}$ )

CEE - custo dos equipamentos elétricos exclusive o motor elétrico (\$),

CEEBT - custo dos equipamentos elétricos de baixa tensão (\$)

$\mathrm{CEQ}_{\mathrm{iZ}}$ - custo $(\$)$ do i-ésimo equipamento no diâmetro ou potência $Z$

CFAS - custo fixo anual do sistema (\$)

CLAT - custo da linha de alta tensão (\$)

$\mathrm{CMa}$ - consumo de energia elétrica medido no ano ( $\mathrm{kWh})$

CMahe - consumo de energia elétrica anual medido no horário especial para irrigantes $(\mathrm{kWh})$

CMCA - custo do motor à combustão e acessórios (\$) 
CME - custo do motor elétrico (\$)

CMLAT - custo médio da linha de alta tensão $(\$ / \mathrm{m})$

CMsfp - consumo de energia elétrica anual medido no período seco no horário fora de ponta $(\mathrm{kWh})$

CMsp - consumo de energia elétrica anual medido no período seco no horário de ponta $(\mathrm{kWh})$

CMshe - consumo de energia elétrica anual medido no periodo seco no horário especial para irrigantes $(\mathrm{kWh})$

CMufp - consumo de energia elétrica anual medido no periodo úmido no horário fora de ponta $(\mathrm{kWh})$

CMup - consumo de energia elétrica anual medido no período úmido no horário de ponta $(\mathrm{kWh})$

CMuhe - consumo de energia elétrica anual medido no período úmido no horário especial para irrigantes $(\mathrm{kWh})$

COCC - custo das obras de construção civil para o sistema (\$)

$\cos \varphi$ - fator de potência nominal do motor elétrico

$\mathrm{Cr}$ - coeficiente de rugosidade médio da fórmula de Hazen-Williams para os tubos do recalque

Cs - coeficiente de rugosidade médio da fórmula de Hazen-Williams para os tubos da sucção

$\mathrm{CSN}$ - custo do sistema novo $(\$)$

CTE - custo do transformador elétrico (\$)

CTr - custo da tubulação de recalque (\$)

CTs - custo da tubulação de sucção (\$)

$\mathrm{C}_{\mathrm{u}} \mathrm{C}$ - custo da unidade de combustivel $\left(\$ / \mathrm{m}^{3}\right)$

CVAS - custo variável anual do sistema $(\$)$

CVE - custo da escavação $\left(\$ / \mathrm{m}^{3}\right)$

CVT - custo das valas para assentamento da adutora (\$)

D - sistema com bomba hidráulica acionada por motor à combustão (diesel)

DC - demanda de potência contratada com a Concessionária de energia elétrica (kW)

DCsfp - demanda de potência contratada no periodo seco no horário fora de ponta com a Concessionária de energia elétrica $(\mathrm{kW})$

DCsp - demanda de potência contratada no periodo seco no horário de ponta com a Concessionária de energia elétrica $(\mathrm{kW})$

DCufp - demanda de potência contratada no período úmido no horário fora de ponta com a Concessionária de energia elétrica $(\mathrm{kW})$ 
DCup - demanda de potência contratada no período úmido no horário de ponta com a Concessionária de energia elétrica $(\mathrm{kW})$

$\mathrm{DM}$ - demanda de potência medida $(\mathrm{kW})$

$\mathrm{d}$ - número de meses completos por ano que o sistema de recalque fica desligado

dsfp - número de meses completos que o sistema de recalque fica desligado no período seco do ano no horário fora de ponta

dsp - número de meses completos que o sistema de recalque fica desligado no período seco do ano no horário de ponta

dufp - número de meses completos que o sistema de recalque fica desligado no período úmido do ano no horário fora de ponta

dup - número de meses completos que o sistema de recalque fica desligado no período úmido do ano no horário de ponta

DV - profundidade da vala $(\mathrm{m})$

EAN - sistema com bomba hidráulica acionada por motor à eletricidade na tarifa azul sem o benefício da Portaria 105 do DNAEE

EAI - sistema com bomba hidráulica acionada por motor à eletricidade na tarifa azul com o beneficio da Portaria 105 do DNAEE

ECN - sistema com bomba hidráulica acionada por motor à eletricidade na tarifa convencional sem o benefício da Portaria 105 do DNAEE

ECI - sistema com bomba hidráulica acionada por motor à eletricidade na tarifa convencional com o benefício da Portaria 105 do DNAEE

EVN - sistema com bomba hidráulica acionada por motor à eletricidade na tarifa verde sem o beneficio da Portaria 105 do DNAEE

EVI - sistema com bomba hidráulica acionada por motor à eletricidade na tarifa verde com o beneficio da Portaria 105 do DNAEE

FC - faturamento anual do consumo de energia elétrica (\$)

FCahc - faturamento do consumo de energia elétrica anual no horário complementar ao especial para irrigantes $(\$)$

FCahe - faturamento do consumo de energia elétrica anual no horário especial para irrigantes $(\$)$

FCsfp - faturamento do consumo de energia elétrica anual no periodo seco no horário fora de ponta (\$)

FCsp - faturamento do consumo de energia elétrica anual no período seco no horário de ponta (\$) 
FCshe - faturamento do consumo de energia elétrica anual no periodo seco no horário especial para irrigantes $(\$)$

FCufp - faturamento do consumo de energia elétrica anual no período úmido no horário fora de ponta $(\$)$

FCup - faturamento do consumo de energia elétrica anual no periodo úmido no horário de ponta $(\$)$

FCuhe - faturamento do consumo de energia elétrica anual no periodo úmido no horário especial para irrigantes $(\$)$

FD - faturamento anual da demanda de energia elétrica (\$)

FDfp - faturamento anual da demanda de energia elétrica no horário fora de ponta (\$)

FDp - faturamento anual da demanda de energia elétrica no horário de ponta (\$)

fdtc - fração de desconto da tarifa de consumo de energia elétrica conforme a Portaria 105 do DNAEE

$f_{\mathrm{BH}}$ - fração do custo da bomba hidráulica nova gasto anualmente com manutenção e reparos para 2000 h de operação

$f_{\mathrm{EE}}$ - fração do custo dos equipamentos elétricos (exceto o motor elétrico) e acessórios novos gasto anualmente com manutenção e reparos para $2000 \mathrm{~h}$ de operação

$\mathrm{f}_{\mathrm{LAT}}$ - fração do custo da linha elétrica de alta tensão nova gasto anualmente com manutenção e reparos

$\mathrm{f}_{\mathrm{MCA}}$ - fração do custo do motor à combustão e acessórios novos gasto anualmente com manutenção e reparos para $2000 \mathrm{~h}$ de operação

$f_{\mathrm{ME}}$ - fração do custo do motor elétrico novo gasto anualmente com manutenção e reparos para $2000 \mathrm{~h}$ de operação

$f_{O C C}$ - fração do custo das obras de construção civil novas gasto anualmente com manutenção e reparos

$\mathrm{f}_{\mathrm{TAH}}$ - fração do custo da tubulação e acessórios novos gasto anualmente com manutenção e reparos para $2000 \mathrm{~h}$ de operação

$f_{\mathrm{TE}}$ - fração do custo do transformador elétrico novo igual ao custo dos acessórios elétricos (isoladores, conectores, cabos, eletrodutos, hastes de aterramento e outros)

$\mathrm{g}$ - aceleração da gravidade, assumida como $9,806 \mathrm{~m} / \mathrm{s}^{2}$

HFr - perda de carga no recalque (m)

HFs - perda de carga na sucção (m) 
HM - altura manométrica do sistema (m)

HR - altura de recalque (m)

$\mathrm{K}_{\mathrm{j}}$ - coeficiente de perda de carga localizada do j-ésimo acessório da sucção

$\mathrm{K}_{\ell}$ - coeficiente de perda de carga localizada do $\ell$-ésimo acessório da sucção

LLAT - comprimento da linha de alta tensão (m)

$\mathrm{Lr}$ - comprimento da tubulação de recalque (m)

Ls - comprimento da tubulação de sucção (m)

$\mathrm{NDa}$ - número de dias por ano de funcionamento do sistema de recalque

NDs - número de dias de funcionamento do sistema de recalque no período seco do ano

$\mathrm{NDu}$ - número de dias de funcionamento do sistema de recalque no período úmido do ano

$\mathrm{n}_{\mathrm{j}}$ - número de unidades do j-ésimo acessório da sucção

$\mathrm{n},-$ número de unidades do j-ésimo acessório da sucção

PA - período de depreciação do sistema (anos)

PF - carga manométrica necessária no final da adutora $(\mathrm{m})$

$Q$ - vazão do sistema adutor $\left(\mathrm{m}^{3} / \mathrm{s}\right)$

$\mathrm{R}$ - fração do custo do sistema novo após o periodo de amortização

$r$ - taxa de juros anual

TCasfp - tarifa de consumo azul no periodo seco no horário fora de ponta $(\$ / \mathrm{kWh})$

TCasp - tarifa de consumo azul no período seco no horário de ponta $(\$ / \mathrm{kWh})$

TCaufp - tarifa de consumo azul no período úmido no horário fora de ponta $(\$ / \mathrm{kWh})$

TCaup - tarifa de consumo azul no periodo úmido no horário de ponta $(\$ / \mathrm{kWh})$

TCc - tarifa de consumo convencional ( $\$ / \mathrm{kWh})$

TCvsfp - tarifa de consumo verde no período seco no horário fora de ponta $(\$ / \mathrm{kWh})$

TCvsp - tarifa de consumo verde no periodo seco no horário de ponta $(\$ / \mathrm{kWh})$

TCvufp - tarifa de consumo verde no período úmido no horário fora de ponta $(\$ / \mathrm{kWh})$

TCvup - tarifa de consumo verde no periodo úmido no horário de ponta $(\$ / \mathrm{kWh})$

TDafp - tarifa de demanda azul no horário fora de ponta (\$)

TDap - tarifa de demanda azul no horário de ponta (\$)

TDc - tarifa de demanda convencional $(\$ / \mathrm{kW})$

TDv - tarifa de demanda verde (\$)

TFa - tempo de funcionamento anual do sistema de recalque (h)

TFd - tempo médio de funcionamento diário do sistema de recalque (h) 
TFdfp - tempo médio de funcionamento diário do sistema de recalque no horário fora de ponta $(\mathrm{h})$

TFdp - tempo médio de funcionamento diário do sistema de recalque no horário de ponta $(\mathrm{h})$

TFdhe - tempo médio de funcionamento diário do sistema de recalque no horário especial para irrigante (h)

TUafp - tarifa de ultrapassagem de demanda azul no horário fora de ponta (\$)

TUap - tarifa de ultrapassagem de demanda azul no horário de ponta (\$)

TUv - tarifa de ultrapassagem de demanda verde (\$)

Vs - velocidade de escoamento na sucção $(\mathrm{m} / \mathrm{s})$

WV - largura da vala (m)

$Z$ - diâmetro (m) para tubos, válvulas e outros mais; ou potência (W) para transformador, chave de partida, motor elétrico e bomba hidráulica do i-ésimo equipamento. 


\title{
DIMENSIONAMENTO ECONÔMICO DE SISTEMAS DE RECALQUE DE ÁGUA EM PROPRIEDADES RURAIS
}

\author{
Autor: JOÃO LUIS ZOCOLER \\ Orientador: Prof. Dr. JOSÉ ANTÔNIO FRIZZONE
}

\section{RESUMO}

Neste trabalho estudou-se o efeito do diâmetro da tubulação nos custos dos sistemas de recalque de água acionados por motores diesel e elétrico, sendo no segundo caso também consideradas as modalidades de tarifação da energia elétrica e os descontos especiais concedidos aos consumidores rurais com sistemas exclusivos para irrigação, a fim de se obter a estimativa e minimização do custo anual total através de um modelo matemático, que foi aplicado em uma situação real de recalque de água para comparação. Finalmente, efetuou-se a análise de sensibilidade do modelo diante da variação do comprimento da linha de alta tensão com o propósito de se verificar o comprimento em que ocorre equiparação de custos de um sistema à combustão com um sistema à eletricidade. Os resultados da aplicação à situação real mostraram que o modelo proposto estimou, em média, $100,9 \%$ do custo anual total do sistema na condição econômica comercial, fato devido, entre outros motivos, ao excelente ajuste da função tipo multiplicativa de custo versus diâmetro ou potência de cada equipamento do sistema. $O$ custo de aquisição e os custos anuais fixos; de bombeamento; de manutenção e reparos; e total do sistema à combustão (diesel) foram superiores ao elétrico em todas as modalidades de tarifação da energia. Entre as modalidades de tarifação da energia elétrica, a horo-sazonal verde foi a que se apresentou mais vantajosa, seguida da convencional e da horo-sazonal azul. Também verificou-se, caso o sistema de recalque fosse utilizado exclusivamente para irrigação, que o proprietário deveria optar pelo desconto especial sobre a tarifa de consumo de energia elétrica durante o periodo das 23 as 5 horas, pois propiciaria, em média, $17,6 \%, 14,8 \%$ e $9,4 \%$ de redução no custo de bombeamento em relação à tarifa normal para as modalidades convencional, horo-sazonal verde e horo-sazonal azul. A análise de sensibilidade dos custos em relação ao comprimento da linha de alta tensão mostrou que, para um comprimento médio de 2.700 metros, o custo de aquisição entre o sistema à combustão (diesel) e à eletricidade seriam equivalentes. Porém, devido ao menor custo operacional, o sistema à eletricidade seria mais vantajoso até o limite de 14.186 metros, que corresponde à equiparação dos custos totais, embora neste caso o seu custo de aquisição fosse $131 \%$ superior. 


\title{
ECONOMIC DESIGN OF THE WATER PUMPING SYSTEMS IN RURAL AREAS
}

\author{
Author: JOÃO LUIS ZOCOLER \\ Adviser: Prof. Dr. JOSÉ ANTÔNIO FRIZZONE
}

\section{SUMMARY}

In this work it was studied the effect of the pipeline diameter in the water pumping system costs using combustion engine and electric engine to estimate and to minimize the annual total cost through mathematics model. In the electric engine case, it was considered the modality of the electric energy rate and the special rebate for rural consumers with exclusive irrigation systems. Too it was applied the model in real situation of pumping water for check it. Finally, it was analyzed the model sensibility according to changed the high voltage line length to verify the value equalize the cost between systems. The results of the application (at real situation) showed that the model has estimated, in average, $100,9 \%$ of the annual total cost system in commercial economical condition, owing to the excellent potential function adjusted of the costs versus diameter equipment or power equipment used in the system. The acquisition cost, fixed annual cost, pumping annual cost, maintenance and repair annual cost and total annual cost of the system using combustion engine was larger electric engine in every modality of the eletric energy rate. The green electric energy rate was cheaper than the conventional electric energy rate and this was cheaper than blue electric energy rate. In exclusive irrigation systems, the consumer ought to choose the special rebate allowed between 23 and $5 \mathrm{~h}$, because it'd reduce the pumping cost $17,6 \%, 14,8 \%$ and $9,4 \%$, in average, from normal electric energy rate for the conventional modality, green modality and blue modality. The analysis of the model sensibility acconding to changed the high voltage line length showed equal acquisition cost between system using combustion engine and electric engine for high voltage line length equal 2700 meters. Because the system using electric engine has smaller operation cost, however, it'd be cheaper till 14186 meters of limit, which corresponds equal total cost between systems, though in this case its acquisition cost'd be $131 \%$ more expensive. 


\section{INTRODUÇÃO}

A implantação de sistemas de recalque de água em propriedades rurais é feita para diversas finalidades, dentre as quais abastecimento de reservatórios destinados à agroindústria (abatedouros, destilarias) e, principalmente, para suprimento de sistemas de irrigação.

A análise econômica de tais sistemas assume grande importância, uma vez que o capital neles empregado é freqüentemente expressivo e seus custos, baseados freqüentemente em período de um ano, podem viabilizar ou não as atividades produtivas que os utilizam.

Os custos de um sistema de recalque são influenciados por muitos parâmetros que, de um modo geral, podem ser divididos em duas categorias: quantitativos e qualitativos. Os quantitativos estão relacionados ao porte do sistema, tendo como principais a vazão; diâmetro, comprimento e pressão necessária no final da adutora; desnível topográfico; potência da bomba hidráulica e do motor para seu acionamento; tempo de funcionamento do motor e, se este for elétrico, também o horário e época do ano que opera; custo do combustivel e das tarifas de energia elétrica; porte das obras de construção civil; comprimento do ramal elétrico; potência da chave de partida e do transformador; além de outros mais que influenciam de maneira menos intensa em tais custos. Os parâmetros qualitativos, como o nome sugere, estão relacionados à qualidade e ao tipo dos equipamentos, ou seja, pode-se optar por equipamentos dotados de melhor qualidade e/ou assistência técnica oferecida pelo fabricante, ou por tipos diferentes de equipamentos, como por exemplo, utilização de motor de combustão interna ao invés do elétrico para acionamento da bomba hidráulica, maior ou menor nível de automação do sistema e assim por diante.

Para uma situação explícita, o diâmetro da adutora é o parâmetro quantitativo mais importante, uma vez que os demais são definidos pelas condições locais, ou seja, a vazão requerida, o comprimento da tubulação, o desnível topográfico, a pressão no final da adutora e o comprimento da linha elétrica de alta tensão (se o bombeamento for com motor à eletricidade), principalmente, estão atrelados aos atributos físicos do local e às exigências dos equipamentos utilizados no final da adutora e, portanto, são constantes. Sendo assim, a variação deste parâmetro acarreta 
alteração nos que dele dependem diretamente, como por exemplo a potência de bomba hidráulica e do motor que a aciona, com conseqüências nos custos do sistema. Por outro lado, o tipo de motor, ou seja, de combustão ou elétrico, é um dos parâmetros qualitativos que também provoca intensa variação nos custos. Para o motor à eletricidade, é importante considerar a modalidade de tarifação da energia elétrica que será aplicada ao consumidor, bem como os custos com a linha de alta tensão, se o ramal elétrico da concessionária estiver distante da estação de bombeamento.

Diante do exposto, este trabalho teve o objetivo de estudar o efeito do diâmetro da tubulação nos custos dos sistemas de recalque de água com bombeamento utilizando motor à combustão e à eletricidade, sendo neste caso também consideradas as modalidades de tarifação da energia elétrica e os descontos especiais concedidos aos consumidores rurais com sistemas exclusivos para irrigação, a fim de se obter a estimativa e minimização do custo anual total através de um modelo matemático. Também aplicou-se o modelo em uma situação real de recalque de água para sua aferição. Finalmente, efetuou-se a análise de sensibilidade do modelo diante da variação do comprimento da linha de alta tensão com o propósito de se verificar o comprimento em que ocorre equiparação de custos de um sistema à combustão com um sistema à eletricidade. 


\section{REVISÃO DE LITERATURA}

Neste item são abordados os custos envolvidos nos sistemas de recalque e seus métodos de cálculo. Segue-se um discorrimento dos principais recursos energéticos para suprimento dos sistemas de recalque, bem como alguns trabalhos que fazem comparações entre custos dos sistemas com bomba hidráulica acionada por motor de combustão e motor elétrico. Finalmente, é dado um enfoque teórico sobre o diâmetro econômico de instalações elevatórias e algumas fórmulas para sua obtenção, com desdobramento dos principais parâmetros envolvidos.

\subsection{Parâmetros econômicos para avaliação de uma instalação elevatória}

"Para fins de análise econômica, o termo custo significa a compensação que os donos dos fatores de produção, utilizados por uma firma para produzir determinado bem, devem receber para que eles continuem fornecendo esses fatores à mesma" (Hoffinann et al, 1983). Os custos são classificados em fixos, que não se alteram com a quantidade produzida, e variáveis, que variam de acordo com o nível de produção da empresa. Para um sistema de recalque, são considerados como custos fixos principalmente a depreciação dos componentes do sistema e a remuneração de capital investido; e como custos variáveis os dispêndios com a energia e com os reparos dos equipamentos e infra-estrutura utilizados na operação do sistema.

\subsubsection{Depreciação e remun eração do capital}

A dep reciação é o custo necessário para substituir os bens de capital quando tomados inúteis pelo desgaste fisico ou quando perdem o valor com o decorrer dos anos devido às inovações técnicas. Quanto à maneira de considerá-la e o método para seu cálculo, segundo Hoffinann et al (1983), diferentes opiniões têm sido sustentadas pelos autores. Uma delas considera que a depreciação se destina a reproduzir, durante o período de utilização do elemento do capital a depreciar, 
uma soma em dinheiro que representa a despesa inicial suportada. $\mathrm{O}$ empresário dela se servirá para substituir o capital sem apelar a seus recursos particulares ou ao crédito. Este método é chamado de fundo de reposição, Coelho (1979), sendo calculado por:

$$
a=\frac{(\mathrm{Cn}-\mathrm{Cu}) \cdot \mathrm{r}}{(1+\mathrm{r})^{\mathrm{PA}}-1}
$$

sendo: a - taxa anual de depreciação;

Cn - valor do bem de capital novo;

$\mathrm{Cu}$ - valor do bem de capital usado;

$r$ - taxa anual de juros;

PA - período de depreciação do capital.

O método de depreciação mais simples considera a desvalorização do bem nos inventários sucessivos durante o período de utilização do bem considerado, não se constituindo, portanto um fundo à parte, ou seja, não ocorre a contagem de juros. É o chamado método linear ou das cotas fixas, sendo calculado por:

$$
a=\frac{C n-C u}{P A}
$$

Outro método de obter a depreciação é o da reavaliação anual que consiste em avaliar o bem de capital cada ano, independentemente do seu custo inicial, baseando-se no valor de revenda. A diferença entre duas avaliações consecutivas seria a depreciação correspondente ao exercício compreendido.

No método da percentagem anual constante a depreciação é uma porcentagem determinada do valor residual do bem. Em certos casos esse método adapta-se bem à diminuição real do valor, mas apresenta o incoveniente de sempre deixar um resíduo.

Caso se deseja distribuir o custo de depreciação mais intensamente nos primeiros anos de uso que nos últimos, o método dos números naturais tem alguma vantagem em relação ao método da porcentagem anual constante, já que deprecia a inversão até o valor final previsto. Neste método, a depreciação de cada ano é uma fração da quantia a amortizar $(\mathrm{Cn}-\mathrm{Cu})$, fração essa cujo denominador é a soma dos números de anos de vida útil do bem, e cujo numerador é o número de anos de vida útil que o bem de capital ainda terá. Assim, se a duração esperada for de 10 anos, soma-se: $10+9+8+7+6+5+4+3+2+1=55$. Deprecia-se no $1^{\circ}$ ano ${ }^{10} / 5$ do total; no $2^{-0}$ ano $\% / 55$ do total; no $3^{0}$ ano ${ }^{8} / 55$ do total; etc. 
O valor da remuneração ou juros sobre o capital investido significa que o empresário renunciou à remuneração que poderia ter obtido pela aplicação de seus capitais em outras altemativas. Essa renúncia representa, para o empresário, o custo a ser considerado. Para que o custo real anual referente aos juros seja o mesmo para qualquer ano, costuma-se calcular os juros sobre o valor médio do capital empatado, ou seja:

$$
\mathrm{j}=\frac{(\mathrm{Cn}+\mathrm{Cu}) \cdot \mathrm{r}}{2}
$$

Para se obter o valor da amortização e da remuneração do capital investido em um sistema de recalque, é necessário, primeiramente, se obter o custo deste sistema, que, de um modo geral, pode ser dividido em custos da tubulação e do conjunto motor-bomba.

O custo da tubulação instalada tem sido representado por diferentes equações para um grande número de autores, tais como Garcez (1946), Camp (1952), Martins (1966), Babbit et al (1967), Mataix (1970), Lencastre (1983) e Azevedo (1991). Basicamente, em todas as equações propostas, o diâmetro é a variável independente e o custo instalado da unidade de comprimento da tubulação é a variável dependente, sendo que o material, a espessura, o revestimento das paredes e as condições de assentamento são os parâmetros, relata Coiado \& Rivelli (1993).

Por sua vez, o custo dos conjuntos motor-bomba e acessórios podem ser expressos como uma função linear da potência destes equipamentos, segundo Coutinho $\&$ Tourasse ${ }^{1}$, citados por Tsutiya (1989) e Azevedo (1991); enquanto para Deb (1978), Bhave (1985) e Chiplunkar et al (1986) tal custo é uma função tipo potencial.

\subsubsection{Reparos e manutenção}

Os reparos e manutenção correspondem ao custo anual necessário para manter o bem de capital em condições de uso. A um maior custo de conservação corresponde, geralmente, uma menor depreciação. As grandes reparações que tem por finalidade reconstituir (em estado de "novo") o bem de capital, no todo ou em parte, são consideradas, via de regra, como despesas extraordinárias. Elas representam um acréscimo de valor ao capital, não devendo, por isso, atribu-

\footnotetext{
${ }^{1}$ COUTINHO, A.; TOURASSE, E. Custo de obras de abastecimento de água e esgotos sanitários. Revista de engenharia sanitária, julho, 1966.
} 
ir-se somente à conta do ano em que foram levados a efeito, mas sim a todos os da respectiva duração. Já as manutenções, reparos ou conservações ordinárias representam despesas do exercício.

Na prática, segundo o Programa Nacional de Irrigação (Brasil) - PRONI (Brasil, 1987), costuma-se calcular os custos da manutenção a partir de valores médios anuais expressos em percentuais sobre o valor de compra do equipamento. A Tabela 1 mostra uma faixa de variação que deve ser aplicada sobre o valor da compra, para a estimativa de manutenção e reparos. É importante notar que a tabela foi elaborada para um periodo de operação anual de 2000 horas. É evidente, portanto, que a vida útil será tanto maior quanto menor for o periodo de operação do equipamento no ano.

Tabela 1. Vida útil e taxas de manutenção de componentes de sistemas de irrigação.

\begin{tabular}{lcc}
\hline \multicolumn{1}{c}{ Componentes } & $\begin{array}{c}\text { Vida útil } \\
\text { (anos) }\end{array}$ & $\begin{array}{c}\text { Manutencão anual } \\
\text { (\% do novo) }\end{array}$ \\
\hline Aspersores fixos & $7-10$ & $5,0-8,0$ \\
Aspersores móveis & $10-15$ & $5,0-8,0$ \\
Bomba centrífuga & $16-25$ & $3,0-5,0$ \\
Bomba eixo vertical & $16-20$ & $4,0-6,0$ \\
Canais permanentes & $15-25$ & $1,0-2,0$ \\
Estação de bombeamento (estrutura) & $20-40$ & $0,5-1,5$ \\
Estruturas de concreto & $15-25$ & $0,5-1,0$ \\
Motor diesel & $10-20$ & $5,0-8,0$ \\
Motor elétrico & $20-25$ & $1,5-2,5$ \\
Poços profundos & $20-30$ & $0,5-1,5$ \\
Reservatórios & - & $1,0-2,0$ \\
Sistematização de terras & - & $1,5-2,5$ \\
Tanque de fertilizantes & $5-10$ & $0,5-1,0$ \\
Tubo de aço (enterrado) & $15-25$ & $0,25-0,50$ \\
Tubo de aço (superficie) & $10-12$ & $1,5-2,5$ \\
Tubo de aço galvanizado (superficie) & $10-20$ & $1,0-2,0$ \\
Tubo de alumínio sob pressão & $10-20$ & $1,5-2,5$ \\
Tubo de cimento amianto (enterrado) & $15-40$ & $0,25-0,75$ \\
Tubo de concreto & $15-25$ & $1,5-2,5$ \\
Tubo de madeira (enterrado) & $10-20$ & $0,25-0,75$ \\
Tubo de polietileno (gotejamento) & $8-10$ & \\
Tubo de PVC (enterrado) & $15-40$ & \\
\hline \hline
\end{tabular}




\subsubsection{Custos com energia para o bombeamento}

Embora os sistemas de recalque nas propriedades agricolas sejam destinados à diversas finalidades, a sua utilização para suprimento de sistemas de irrigação é sem dúvida a de maior destaque. Conforme Scaloppi (1985), a irrigação é considerada uma técnica de grande utilização de energia na agricultura. Um estudo baseado em dados de 1972, desenvolvido pelo Departamento de Engenharia Agrícola da Universidade da Califórnia, Davis, revelou que a irrigação requer aproximadamente $13 \%$ da energia diretamente utilizada na agricultura desse Estado americano. Esta quantidade de energia é superior àquela utilizada para estabelecer a cultura e desenvolver todas as práticas culturais. $\mathrm{O}$ mesmo autor, citando Knutson et $\mathrm{al}^{2}$, revela que a maior parte desta energia tem a finalidade de trazer a água até a área irrigada, quer retirando-a dos aquíferos subterrâneos (43\%), quer dos recursos superficiais, como reservatórios e rios (41\%). Os restantes $16 \%$ são utilizados para pressurizar os sistemas de distribuição de água na área irrigada. A maior parte dessa energia utilizada para a distribuição de água (91\%) é requerida para pressurizar sistemas por aspersão, apesar da área irrigada por estes sistemas representar apenas $17 \%$ da área tơtal irrigada nesse estado americano.

O custo da energia utilizada em irrigação depende, segundo o mesmo autor, da fonte de energia disponivel e da eficiência da unidade de potência considerada. No caso de motores elétricos, basta multiplicar o custo do $\mathrm{kWh}$ pelo consumo de energia em MJ e dividir por 3,6 (1 kWh $=3,6 \mathrm{MJ}$ ). Em se tratando de motores de combustão interna, deve-se conhecer o poder calorifico do combustivel utilizado e o rendimento global de conversão correspondente. A Tabela 2 apresenta tais informações para alguns combustíveis.

A equação geral da energia requerida no bombeamento de água pode ser descrita por:

$$
\mathrm{E}=\mathrm{V} \cdot \mathrm{HM} \cdot \gamma / \eta
$$

sendo: $\mathrm{E}$ - energia requerida pela unidade de bombeamento, expressa em Joule (J);

$\mathrm{V}$ - volume de água bombeado, em metros cúbicos $\left(\mathrm{m}^{3}\right)$;

HM - altura manométrica total, em metros (m);

$\gamma$ - peso especifico da água, considerado 9806,65 Newtons por metro cúbico $\left(\mathrm{N} / \mathrm{m}^{3}\right)$;

\footnotetext{
${ }^{2}$ KNUTSON, G.D.; CURLEY, R.G.; ROBERTS, E.B.; HAGAN, R.M.; CERVINKA, V. Pumping energy requeriments for irrigation in Califórnia. Special Publication 3215, Div. Agric. Sci., University of Califórnia, 1978. $54 \mathrm{p}$.
} 
$\eta$ - eficiência global da unidade de bombeamento, incluindo a bomba hidráulica, a unidade de potência e o sistema de transmissão.

Uma simples análise da equação (2.4) permite estabelecer que a energia requerida em estações de bombeamento pode ser reduzida através da redução do volume de água bombeado (V), da altura manométrica total (HM) e do aumento da eficiência global de bombeamento $(\eta)$. Baseadas nesta equação, três categorias de práticas para economizar energia podem ser apresentadas: a) redução do volume de água requerido através de um melhor manejo da água de irrigação; escolha criteriosa da quantidade de água a ser aplicada, utilizando funções de produção; dimensionamento e operação para a obtenção de elevados níveis de eficiência de aplicação de água etc.; b) redução da altura manométrica total através da utilização de sistemas com menores exigência de pressão; redução das perdas de pressão no sistema de condução e distribuição de água através de tubulações e acessórios etc.; c) aumento da eficiência global de bombeamento, através do aumento das eficiências das unidades de potência, das bombas hidráulicas e dos mecanismos de transmissão; dimensionamento criterioso do conjunto motobomba; operação na faixa de máxima eficiência hidráulica etc.

Tabela 2. Características energéticas dos principais combustíveis líquidos e gasosos utilizados em unidades de bombeamento para irrigação ${ }^{(a)}$.

\begin{tabular}{|c|c|c|c|}
\hline Combustível & Poder calorífico & Massa específica & Rendimento global \\
\hline Diesel & $41,66 \pm 0,63 \mathrm{MJ} / \mathrm{kg}^{(\mathrm{b})}$ & $0,85-0,88 \mathrm{~kg} / \mathrm{dm}^{3}$ & $0,30^{(\mathrm{c})}$ \\
\hline Gasolina & $42,91 \pm 0,63 \mathrm{MJ} / \mathrm{kg}$ & $0,84-0,86 \mathrm{~kg} / \mathrm{dm}^{3}$ & $0,27^{(\mathrm{d})}$ \\
\hline Álcool hidratado a $95 \%$ & $25,31 \mathrm{MJ} / \mathrm{kg}$ & $0,81 \mathrm{~kg} / \mathrm{dm}^{3}$ & $0,34^{(\mathrm{d})}$ \\
\hline Metano & $35,92 \mathrm{MJ} / \mathrm{m}^{3}$ & $0,72 \mathrm{~kg} / \mathrm{dm}^{3}$ & $0,20^{(\mathrm{c})}$ \\
\hline Butano & $123,76 \mathrm{MJ} / \mathrm{m}^{3}$ & $2,70 \mathrm{~kg} / \mathrm{m}^{3}$ & 0,20 \\
\hline Propano & $92,99 \mathrm{MJ} / \mathrm{m}^{3}$ & $2,02 \mathrm{~kg} / \mathrm{m}^{3}$ & 0,20 \\
\hline Gasogênio & $4,81-5,23 \mathrm{MJ} / \mathrm{dm}^{3}$ & $1,1-1,2 \mathrm{~kg} / \mathrm{m}^{3}$ & 0,20 \\
\hline Gás natural seco & $25,12-33,49 \mathrm{MJ} / \mathrm{dm}^{3}$ & $\sim 0,7 \mathrm{~kg} / \mathrm{m}^{3}$ & 0,20 \\
\hline Gás natural úmido & $29,31-56,52 \mathrm{MJ} / \mathrm{dm}^{3}$ & $0,7-1,0 \mathrm{~kg} / \mathrm{m}^{3}$ & 0,20 \\
\hline Biogás $(65-67 \% \text { metano })^{(e)}$ & $20,49-26,08 \mathrm{MJ} / \mathrm{dm}^{3}$ & $\sim 0,7 \mathrm{~kg} / \mathrm{m}^{3}$ & 0,20 \\
\hline
\end{tabular}

Os custos decorrentes do consumo de energia elétrica, conforme Tsutiya (1989), podem ser calculados pela equação:

$$
\mathrm{C}_{\mathrm{c}}=8760 \cdot \text { B.P. } \mathrm{C}_{\mathrm{uc}}
$$

sendo: $C_{c}$ - custo total anual de consumo de energia elétrica $(\$)$; 
B - fator que reflete o tempo de funcionamento dos conjuntos motor-bomba (se contínuo B $=1$ );

$\mathrm{P}$ - potência dos conjuntos motor-bomba para cada diâmetro $(\mathrm{kW})$;

$\mathrm{C}_{\mathrm{uc}}$ - custo unitário do consumo da energia elétrica $(\$ / \mathrm{kWh})$.

Quando as cargas solicitadas pela elevatória são maiores que $75 \mathrm{kVA}$, as concessionárias cobram também o custo da demanda de potência, dado por:

$$
\mathrm{C}_{\mathrm{d}}=12 \cdot \mathrm{P} \cdot \mathrm{C}_{\mathrm{ud}}
$$

sendo: $C_{d}$ - custo anual de demanda de potência $(\$)$;

$\mathrm{C}_{u d}$ - custo unitário da demanda de potência (\$/kWh.mês).

No caso de estações elevatórias de sistemas de abastecimento de água de pequeno e médio portes, Tsutiya (1989) também apresenta uma série de alternativas que contribuem com a redução do custo de energia elétrica, entre as quais:

i) Enquadramento na estrutura tarifária mais adequada, bem como o equilibrio entre as demandas contratada e registrada, a fim de se evitar o pagamento de uma demanda não utilizada, ou ainda, no caso de tarifação horo-sazonal, da demanda de ultrapassagem;

ii) Deve-se evitar a utilização do conjunto motor-bomba no horário de ponta. Se for comparado o custo do bombeamento no horário de ponta em relação ao bombeamento fora de ponta, o acréscimo nos custos variará de $2,8 \%$ a $100 \%$, dependendo do fator de carga da instalação, do esquema operacional adotado e do tipo de tarifa;

iii) Correção do fator de potência pela instalação de banco de capacitores; modificação do padrão de entrada de energia elétrica de baixa para alta tensão, pois o consumo com tarifa em alta tensão geralmente é mais econômico; e elevação do fator de carga para 0,6 ou mais através da adequação dos conjuntos motor-bomba;

iv) Devem ser utilizados nos conjuntos motor-bomba os variadores de rotação para controle da vazão em sistemas onde o bombeamento de água é dirigido diretamente ao consumidor. Propõe-se a utilização do variador hidrocinético quando a elevatória se situa em regiões periféricas e do inversor de freqüência nas demais regiões;

v) A forma e as dimensões do poço de sucção não devem permitir a formação de vórtices e, consequentemente entrada de ar na tubulação de sucção;

vi) Nas adutoras de água bruta, há necessidade de se prever dispositivos que possibilitem a introdução de equipamentos de limpeza nos tubos, independentemente da qualidade da água. $\mathrm{O}$ método mais adotado pelas entidades de saneamento para limpeza é o da passagem de equipamen- 
tos que removem as incrustações através de raspagem. A escolha do tipo da peça para efetuar a limpeza depende do material que caracteriza a tubulação e da incrustação existente. Nos tubos metálicos revestidos, tubos de PVC, concreto e fibrocimento utiliza-se o "polly-pig", equipamento dotado com fita de material abrasivo para não danificar a parte intema da tubulação. No caso de tubos metálicos não revestidos emprega-se o "polly-pig" com escovas de aço, ou ainda o raspador de arraste hidráulico. A utilização destes equipamentos tem a vantagem de praticamente não interromper a operação normal da adutora, devido à rapidez do serviço, além do custo reduzido. Na primeira limpeza das adutoras de água bruta de São José dos Campos (SP), que são constituídas de ferro dúctil com e sem revestimento (ambas com $3100 \mathrm{~m}$ extensão e $600 \mathrm{~mm}$ de diâmetro) o coeficiente "C" da fórmula de Hazen-Williams passou de 59 para $119 \mathrm{e}$ de 79 para 109, respectivamente.

Até 1969, o Departamento Nacional de Águas e Energia Elétrica - DNAEE, concedia um subsídio de $80 \%$ nas tarifas de energia elétrica para os serviços públicos de abastecimento de água e disposição de esgotos. Todavia, esse desconto com o passar do tempo foi gradualmente reduzido chegando a $15 \%$ em 1989.

Conforme Tsutiya (1989) e Coiado e Rivelli (1993), estudos mostram que o indice da variação percentual acumulada - IVPA - para as tarifas de energia elétrica tiveram, a partir de 1977, valores maiores que o índice geral de preços (1704,88\% a mais no período de 1977 a 1988), enquanto a variação dos custos da adutora mantiveram-se com indices próximo do indice geral de preços (140\% a mais no mesmo periodo). Devido à previsão de crise no fomecimento de energia elétrica nos próximos anos, ainda sem equacionamento a curto e médio prazos, o govemo vem aumentando as tarifas de energia acima dos indices inflacionários, de modo a forçar o usuário a promover o uso eficaz da energia elétrica. Como consequência, o custo da energia elétrica deverá ter influência bem mais significativa na escolha do diâmetro econômico de instalações de recalque.

"Dados do Ministério das Minas e Energia estimavam, em 1996, que o país - Brasil perde $R \$ 2,5$ bilhões por ano com desperdício de energia elétrica. Da quantia, $R \$ 1,5$ bilhão fica por conta dos hábitos inadequados e equipamentos ineficientes." (Folha de São Paulo, 1997a).

Em matéria publicada pela Folha de São Paulo (1997b), o presidente das Centrais Elétricas Brasileiras - ELETROBRÁS, Firmino Sampaio Neto disse que para evitar o racionamento nas regiões Sul, Sudeste e Centro-Oeste, afetadas por dois blecautes (causados, segundo o presidente, pela sobrecarga no consumo) nas noites dos dias 24 e 25 de abril de 1997, a empresa estu- 
da a adoção de medidas para a "racionalização do uso" de energia. Uma das medidas em análise é aumentar a tarifa nos horários de pico (principalmente, entre 18 e $19 \mathrm{~h}$ ).

Os três últimos parágrafos enfatizam a importância de se utilizar a energia de modo racional, fato que exige também entre os projetistas de sistemas de adução em recalque uma análise pormenorizada dos custos da energia nestes sistemas.

\subsection{Recursos energéticos para acionamento dos sistemas de recalque e análise com- parativa de custos de sistemas a óleo diesel è eletricidade}

O PRONI (Brasil, 1987) relaciona algumas fontes de energia para acionamento de sistemas de recalque, dentre as quais: $i)$ hidráulica, ii) diesel, iii) gás proveniente da biodigestão, iv) eólica, v) solar e vi) álcool.

i) Por ser um país de enorme potencial hidráulico, não é supreendente ser esta a principal de suas fontes energéticas. A energia gerada pelas centrais hidrelétricas é transportada através de linhas de transmissão, subestações e redes de distribuição, até chegar aos consumidores. Locais distantes da rede elétrica coberta pelo Sistema Interligado das Concessionárias poderão apelar para as Pequenas Centrais Hidrelétricas ( $\mathrm{PCHs}$ ), que alimentam sistemas isolados e destinam-se ao fornecimento de energia elétrica a grupos de consumidores situados num raio de apenas alguns quilômetros. Estas PCHs, por independerem do sistema interligado, apresentam algumas vantagens como o custo operacional menor e não vinculação ao sistema tarifário nacional. Como testemunho dessa bem sucedida experiência, o autor destaca as 19 pequenas usinas que operam na área da CPFL - Companhia Paulista de Força e Luz - em São Paulo, com uma capacidade instalada de $118.052 \mathrm{~kW}$, em que grande parte dos irrigantes dessa região utilizam motobombas elétricas para acionar seus sistemas de irrigação. Outro testemunho desta eficiência é o programa de PCHs desenvolvido pelo Departamento Municipal de Eletricidade de Poços de Caldas, que pôs em funcionamento sete pequenas centrais com total de $21.849 \mathrm{~kW}$;

ii) Os motores a óleo diesel são úteis nas regiões distantes do sistema elétrico da concessionária local e nas quais, além disso, a implantação de uma $\mathrm{PCH}$ também é inviável. Neste caso, o motor diesel é mais econômico que o motor elétrico quanto ao custo inicial necessário, pois o motor elétrico exige a construção da rede elétrica para ligar o equipamento ao sistema de uma concessionária local, embora em si, este seja mais econômico que o diesel correspondente; 
iii) O biogás, que é uma fonte energética oriunda do processo de fermentação de matérias orgânicas diluídas em água nos biodigestores, é uma fonte altemativa para acionar motores de combustão e, consequentemente, sistemas de recalque. Como exemplo, são citadas pelo autor as experiências feitas em São Roque (SP), no projeto "Horta Comunitária". Na Índia e China, os biodigestores são muito utilizados, uma vez que são grandes as distâncias entre as propriedades rurais e as fontes tradicionais de energia, além do fato destes equipamentos formecerem biofertilizantes, que são os resíduos da digestão anaeróbica;

iv) Em alguns locais onde a velocidade do vento supera os valores mínimos considerados viáveis $(25$ a $30 \mathrm{~km} / \mathrm{h})$, pode-se utilizar um aerogerador, que é um dispositivo que gera energia elétrica por intermédio de uma turbina eólica e um gerador. A energia é utilizada para mover motobombas. Levantamentos feitos sobre o potencial eólico brasileiro revelam que certas áreas do Rio Grande do Sul, Mato Grosso do Sul e litorais do Rio de Janeiro, do Espírito Santo e do Nordeste são as mais adequadas à aplicação deste equipamento;

v) As baterias de células fotovoltaicas transformam a energia solar em calor e vapor para geração de energia elétrica que, assim, podem alimentar pequenos sistemas de irrigação em regiões onde a insolação é intensa. A CESP - Companhia Energética de São Paulo - desenvolveu um protótipo que funciona no Museu da Ciência e Tecnologia, em São Paulo. Conforme o autor, inúmeros exemplos concretos desta aplicação encontram-se na Europa e África, onde sistemas de irrigação são acionados por tais dispositivos, com excelentes resultados;

vi) Algumas usinas do interior do Estado de São Paulo, como a São Martinho, em Pradópolis, tem desenvolvidos testes com motores a álcool para distribuição, por aspersão, do vinhoto (fertilizante), através de conjuntos adaptados para este fim, produzindo resultados animadores.

Embora existam estas e outras fontes de energia para acionamento dos motores, a hidreletricidade e o diesel são as mais utilizadas no Brasil e, portanto, mais enfatizadas nos trabalhos envolvendo custos de sistemas de recalque. Sendo assim, o mesmo autor relata que as principais vantagens econômicas dos motores elétricos sobre os diesel de mesma potência são: a) maior economia operacional; b) maior vida útil; c) maior rendimento; d) partida instantânea nos dias mais frios; e) fácil disponibilidade de energia junto ao sistema elétrico quando o ponto de bombeamento estiver próximo ao sistema elétrico da concessionária, bastando solicitar a ligação dos equipamentos, enquanto o diesel requer gastos de transporte e armazenamento do óleo; f) funcionamento "limpo", isto é, não polui a atmosfera com gases da combustão, nem provoca poluição 
sonora na circunvizinhança. O motor diesel, no entanto, possui maior mobilidade que o elétrico, sendo uma vantagem que permite ao mesmo o deslocamento por vários pontos de tomada de água. Todavia, esta vantagem deixa de existir se o motor elétrico mais a bomba hidráulica e os dispositivos de comando, de um lado, e o transformador, de outro lado, forem acoplados às respectivas carretas móveis, cujo projeto foi desenvolvido por técnicos da CPFL. Para isso, basta construir os ramais, colocar as chaves corta-circuitos e preparar o acoplamento da carreta móvel do transformador. Graças a essas adaptações, foi possível atender às necessidades de irrigação de cerca de 15 plantadores de batata na região de Itatiba (SP).

Em 1973, com a considerável elevação dos preços do petróleo importado, a ELETROPAULO - Eletricidade de São Paulo S/A - promoveu a substituição de 400 conjuntos motobombas acionados a óleo diesel por conjuntos acionados à eletricidade em propriedades rurais que pertenciam a sua área de concessão, relata Gonçalves et al (1986).

Mesmo que os custos operacionais da irrigação que utiliza energia elétrica trifásica sejam inferiores aos que utiliza óleo diesel, Tew et $\mathrm{al}^{3}$, citados por ALVES (1990) constataram que as propriedades rurais do Estado da Georgia - U.S.A., situadas em locais com possibilidade de irrigação, tiveram pequena adesão a esta modalidade quando os custos das redes trifásicas foram financiados pelos próprios proprietários. Também constataram que as economias geradas por esta modalidade em relação ao diesel permitem que propriedades rurais com cerca de 20 ha possam financiar redes trifásicas de até $1900 \mathrm{~m}$ de comprimento, e propriedades com 40 a 60 ha possam financiar tais redes de até 2900 e $4500 \mathrm{~m}$ de comprimento, respectivamente. Redes acima desses comprimentos devem ser subsidiadas pelo governo, cooperativas ou companhia concessionária.

Scaloppi (1985) realizou um estudo comparativo do consumo e custos com bombeamento para recalcar $1000 \mathrm{~m}^{3}$ de água a uma altura manométrica total de $1 \mathrm{~m}$, para motores elétricos e de combustão interna acoplados a uma bomba hidráulica com $70 \%$ de eficiência. $\mathrm{O}$ rendimento assumido para o motor elétrico foi $\mathbf{8 8 \%}$. Considerando-se os valores médios de poder calorífico, massa específica e rendimento do motor para os combustíveis apresentados na Tabela 2, foram obtidos os resultados mostrados na Tabela 3.

\footnotetext{
${ }^{3}$ TEW, B.V. et al. Market failure in multiphase electric power development for agricultural irrigation. Georgia: The University of Georgia, 1983. 15p.
} 
Tabela 3. Energia requerida, consumo e custo para recalcar $1000 \mathrm{~m}^{3}$ de água a uma altura manométrica de $1 \mathrm{~m}$, para motores elétricos e de combustão interna.

\begin{tabular}{lccc}
\hline $\begin{array}{c}\text { Fonte } \\
\text { energética }\end{array}$ & $\begin{array}{c}\text { Energia } \\
(\mathbf{M J})\end{array}$ & Consumo & $\begin{array}{c}\text { Custo (a) } \\
\text { (Cr\$) }\end{array}$ \\
\hline Eletricidade & 15,92 & $4,42 \mathrm{kWh}$ & 330,00 \\
Diesel & 46,70 & $1,30 \mathrm{dm}^{3}$ & $1.976,00$ \\
Gasolina & 51,89 & $1,42 \mathrm{dm}^{3}$ & $3.081,00$ \\
Álcool hidratado a $95 \%$ & 41,20 & $2,01 \mathrm{dm}^{3}$ & $2.834,00$ \\
Gás natural & 70,05 & $1,63 \mathrm{~m}^{3}$ & $-(\mathrm{b})$ \\
Gasogênio & 70,05 & $13,95 \mathrm{~m}^{3}$ & $-(\mathrm{b})$ \\
Biogás (65-67\% metano) & 70,05 & $3,01 \mathrm{~m}^{3}$ & $-(\mathrm{b})$ \\
\hline \hline
\end{tabular}

(a) Valores de maio de 1985: 1US\$ $=\operatorname{Cr} \$ 6.167,42 ; 1 \mathrm{kWh}=\operatorname{Cr} \$ 74,58$ (instalado em baixa tensão, fora do horário de pico); $1 \mathrm{dm}^{3}$ óleo diesel $=\operatorname{Cr} \$ 1.520,00 ; 1 \mathrm{dm}^{3}$ gasolina $=\operatorname{Cr} \$ 2.170,00 ; 1$ $\mathrm{dm}^{3}$ álcool hidratado $=\mathrm{Cr} \$ 1.410,00$

(b) Preços não disponiveis.

Com os dados da Tabela 3 é possível avaliar a acentuada diferença da quantidade de energia requerida, do consumo e do custo para cada fonte energética considerada. A consideração mais favorável, neste caso, está associada aos maiores índices de rendimento global dos motores, do poder calorífico do combustível e ao menor custo da energia requerida. $O$ elevado rendimento global dos motores elétricos associados ao reduzido custo do $\mathrm{kWh}$ utilizado no periodo fora de pico reduzem o custo de energia em muito maior proporção que qualquer outro combustível líquido ou gasoso utilizado.

Simas ${ }^{4}$, citado por Melo (1993) também comparou o custo anual total, representado pela soma dos custos fixos, operacionais e de manutenção, dos motores diesel com o dos motores elétricos, em função das horas anuais de operação. A vantagem dos motores diesel ficou limitada ao intervalo de até 450 horas anuais de funcionamento.

Mello ${ }^{5}$ et al, citado por Alves (1990), em estudo econômico do emprego de óleo diesel versus energia elétrica em sistemas de irrigação, enfatizam a economicidade desta última fonte de energia no custo operacional para culturas irrigadas do tipo "cebola de muda", "tomate envarado"

\footnotetext{
${ }^{4}$ SIMAS, J.R. Politica nacional de irrigação: perspectivas para o ano 2000 - demandas energéticas e hídricas. Fortaleza, DNOCS, 1988. 34p.

${ }^{5}$ MELLO, N.T.C. et al. Estudos econômicos do emprego da irrigação com energia fóssil versus energia elétrica. São Paulo, Instituto de Economia Agrícola, 1986. 29p. (Relatório).
} 
e "batata das águas". Em contrapartida, os mesmos autores ressaltam um fato que merece a atenção dos agentes envolvidos em políticas de estímulo à expansão do consumo de energia elétrica na irrigação, ou seja, o capital fixo necessário por unidade de área toma-se elevado em se tratando de pequenos produtores rurais. Assim, quando o consumo de energia tiver uma pequena participação no custo total, a ocorrência de diminuição dos custos variáveis pode ser pouco significativa a ponto de não ser suficientemente estimulante o emprego de energia elétrica em detrimento ao óleo diesel nestes sistemas.

Um estudo sobre o interesse dos proprietários ou ocupantes rurais em ingressar no programa de irrigação do Distrito de Santa Terezinha (MS) e, também, o tipo de energia a ser empregada foi realizado por Alves (1990). O autor constatou que o tamanho máximo das propriedades pesquisadas é 52 ha e o mínimo é 12 ha, sendo que o tamanho médio é 28,73 ha, podendo ser tipificadas como muito pequenas conforme Moreira ${ }^{6}$. Embora os conjuntos a óleo diesel para todas as potências estudadas sejam mais onerosos que os à eletricidade, quando ao valor dos últimos são somados os valores da rede de distribuição mais o transformador, então seus custos tornam-se maiores que os conjuntos a óleo diesel. O comprimento médio da rede de distribuição de energia elétrica que o irrigante deveria instalar foi calculado em $1.464 \mathrm{~m}$, sendo seu custo, estimado pela Cooperativa de Energização Rural da Grande Dourados - GERGRAND, igual a 8.351,33 BTNs (US\$ 6.279,20) mais o transformador, o que segundo o autor não condiz com a realidade econômica destes pequenos produtores. Sendo assim, as conclusões do estudo foram: a) $46,67 \%$ das propriedades que reúnem as melhores condições de adotar técnicas de irrigação empregando eletricidade, manifestaram desinteresse em se ingressarem no programa; b) a implantação do programa de irrigação utilizando eletricidade depende da adequação técnica do sistema de distribuição desta forma de energia; c) considerando o comprimento mínimo de $1.022 \mathrm{~m}$ da rede primária de distribuição, o volume de investimentos em sistemas a diesel é menor que à eletricidade para motores nas potências 50,75 e $100 \mathrm{hp}$; d) para motores com potência de $20 \mathrm{hp}$ os gastos com energia elétrica em sistemas de irrigação são pouco superiores aos com diesel; e) para motores com potência de 50 e 75 hp os gastos com óleo diesel são superiores aos com energia elétrica; f) considerando os gastos médios anuais com consumo de energia, manutenção, depreciação e juros sobre investimentos, o emprego de energia elétrica retringe-se aos motores de $20 \mathrm{hp}$, se o comprimento

\footnotetext{
${ }^{6}$ MOREIRA, R.J. Contribuição à análise de investimentos e poupança na agricultura brasileira. Osasco, 1973. 203p. Tese (Doutorado) - Faculdade Municipal de Ciências Econômicas e Administrativas de Osasco.
} 
da linha de alta tensão for no máximo igual a $1.180 \mathrm{~m}$, sendo indicado o emprego do óleo diesel para motores de 50 e $75 \mathrm{hp} ; \mathbf{g}$ ) substituindo-se a eletricidade pelo óleo diesel no programa, consegue-se um incremento de $200 \%$ no número de propriedades rurais que reúnem condições imediatas para ingressarem no programa.

Melo (1993) conduziu no Estado de Minas Gerais um trabalho para determinar e analisar custos da irrigação em 237 sistemas de irrigação, sendo 108 do tipo convencional semiportátil, 86 do tipo pivô central e 43 do tipo autopropelido. Algumas das conclusões foram: os custos de investimento, variáveis e totais dos sistemas com motor diesel foram sempre superiores aos dos que utilizavam energia elétrica; a energia foi o item de maior participação na composição dos custos variáveis, principalmente nos sistemas com motor diesel, onde se obteve a relação custos da energia diesel / custo da energia elétrica $=4,2$; a vazão total e o comprimento da adutora por unidade de área explicaram as maiores variações nos custos de investimento; e a área irrigada, horas anuais de operação e investimento por hectare foram as principais variáveis a interferir nos custos operacionais da irrigação.

Frizzone et al (1994) compararam os custos da irrigação em cultura do feijão, utilizando-se o sistema de irrigação por aspersão tipo pivô central para uma área de 91,3 ha, acionado à energia elétrica e a óleo diesel. Fizeram 12 simulações referentes a 12 épocas de semeadura no ano, admitindo o ciclo da cultura de 82 dias. Os dados utilizados referem-se a um projeto da EPAL Engenheiros Associados S/C Ltda, na região de Barreiras (BA). No cálculo dos custos variáveis para o sistema elétrico foram considerados dois períodos para aplicação de água à cultura: um com desconto de $90 \%$ da tarifa de consumo para a operação entre 23 e 5 horas, o que contempla necessidades de irrigação de até 180 horas mensais; o outro periodo, correspondente às horas excedentes com tarifa normal. No cálculo dos custos fixos foram considerados a depreciação do sistema e a remuneração do capital nele investido. Na depreciação utilizou-se o método do fundo de amortização e na estimativa da remuneração trabalhou-se com o valor médio do sistema novo. Considerando-se que o sistema de irrigação é utilizado para produzir duas safras de feijão por ano, sendo uma com semeadura em maio e outra em outubro, verificou-se que o sistema acionado a diesel resulta num custo anual de irrigação $72,57 \%$ superior ao sistema acionado à energia elétrica (para 1.218,4 horas de irrigação). O resumo dos resultados para as duas safras de feijão por ano foram: 
i) Custo anual do consumo efetivo de energia elétrica:

Semeadura em maio

$1.780,30$

Semeadura em outubro

Subtotal

ii) Custo da demanda de energia elétrica:

Semeadura em maio 973,10

Semeadura em outubro

Subtotal

iii) Custo anual da energia elétrica (adicionando-se 13\% de ICMS)

iv) Custo fixo anual do sistema à eletricidade $13.372,18$

v) Custo total da irrigação à eletricidade $17.966,28$

vi) Custo anual do consumo de diesel:

Semeadura em maio

$10.011,70$

Semeadura em outubro

$4.715,10$

vii) Custo anual do consumo de diesel

$14.726,80$

viii) Custo fixo anual do sistema acionado a óleo diesel

$16.277,32$

ix) Custo total da irrigação com sistema acionado a óleo diesel

$31.004,12$

Com estes resultados e outros mais obtidos, as conclusões foram: a) os custos variáveis da irrigação, representados pelo custo da energia, foram sempre maiores no sistema com motor diesel que no sistema com motor elétrico; b) o custo do milímetro de água aplicado no sistema à eletricidade diminuiu exponencialmente com o aumento do número de horas de irrigação e foi sempre inferior ao diesel; c) a diferença entre os custos energéticos no sistema a diesel e elétrico cresceu com o aumento da lâmina de água aplicada; d) a distribuição da necessidade de irrigação durante o ciclo da cultura afetou o custo do milímetro de água aplicado pelo sistema à eletricidade.

Sentelhas et al (1997) estudaram a viabilidade do uso da tarifação especial para irrigação notuma nas regiões Sudeste (SE), Centro-Oeste (CO) e Nordeste (NE), cujas evapotranspirações máximas (ETm) consideradas foram $4 \mathrm{~mm}, 6 \mathrm{~mm}$ e $8 \mathrm{~mm}$, respectivamente. Baseado nos descontos regionais (SE: 60\%, CO: 67\% e NE: 73\%) e no custo do $\mathrm{kWh}$, elaborou-se uma tabela dos custos da energia para estas regiões, considerando-se somente o grupo de baixa tensão $(\leq 2,3$ $\mathrm{kV}$ ). Nas simulações feitas para a adoção da tarifação noturna incluiu-se o custo de aquisição do registrador de energia para irrigantes, o "REP - Registrador Eletrônico Programável", especial- 
mente utilizado para esse fim, de valor $\mathrm{R} \$ 1.500,00$; bem como o custo da instalação da rede elétrica exclusiva para o registrador, com $1 \mathrm{~km}$ de extensão, de valor R\$4.000,00. Os índices econômicos utilizados foram o custo total anual por hectare (CTA/ha) e o custo de aquisição por hectare $(\mathrm{CA} / \mathrm{ha})$, que podem ser observados nas Tabelas 4 e 5 , respectivamente.

Tabela 4. Custo total anual por hectare (CTA/ha) para as combinações mais econômicas de linha lateral (LL), linha principal (LP) e variação de pressão (VP), para irrigações diurnas, com tarifação normal da energia elétrica, e noturnas, com tarifação especial da energia elétrica.

\begin{tabular}{ccccccc}
\hline $\begin{array}{c}\text { Região } \\
\text { Horário }\end{array}$ & $\begin{array}{c}\text { LL } \\
\text { (polegadas) }\end{array}$ & $\begin{array}{c}\text { LP } \\
\text { (polegadas) }\end{array}$ & $\begin{array}{c}\text { VP } \\
\text { (mca) }\end{array}$ & $\begin{array}{c}\text { Area } \\
\text { (ha) }\end{array}$ & $\begin{array}{c}\text { CTA/ha } \\
\text { (RS) }\end{array}$ & $\begin{array}{c}\text { Velocidade } \\
\text { na LP (m/s) }\end{array}$ \\
\hline SE - Dia & 4 & 8 & 40 & 45,73 & 313,19 & 1,23 \\
SE - Noite & 4 & 6 & 40 & 16,53 & 343,41 & 2,18 \\
CO - Dia & 4 & 8 & 20 & 23,40 & 729,41 & 0,94 \\
CO - Noite & 4 & 6 & 40 & 11,46 & 575,04 & 2,18 \\
NE - Dia & 4 & 8 & 20 & 17,56 & $1.745,89$ & 0,94 \\
NE - Noite & 4 & 6 & 40 & 8,92 & 919,07 & 2,18 \\
\hline \hline
\end{tabular}

Tabela 5. Custo de aquisição por hectare (CA/ha) para as combinações mais econômicas de linha lateral (LL), linha principal (LP) e variação de pressão (VP), para irrigações diurnas, com tarifação normal da energia elétrica, e notumas, com tarifação especial da energia elétrica.

\begin{tabular}{ccccccc}
\hline $\begin{array}{c}\text { Região } \\
\text { Horário }\end{array}$ & $\begin{array}{c}\text { LL } \\
\text { (polegadas) }\end{array}$ & $\begin{array}{c}\text { LP } \\
\text { (polegadas) }\end{array}$ & $\begin{array}{c}\text { YP } \\
(\mathbf{m c a})\end{array}$ & $\begin{array}{c}\text { Area } \\
\text { (ha) }\end{array}$ & $\begin{array}{c}\text { CA/ha } \\
\text { (RS) }\end{array}$ & $\begin{array}{c}\text { Velocidade } \\
\text { na LP (m/s) }\end{array}$ \\
\hline SE - Dia & 4 & 5 & 20 & 35,09 & 552,40 & 2,41 \\
SE - Noite & 4 & 6 & 40 & 16,53 & $1.663,25$ & 2,18 \\
CO - Dia & 4 & 5 & 20 & 23,40 & 750,12 & 2,41 \\
CO - Noite & 4 & 6 & 40 & 11,46 & $2.330,03$ & 2,18 \\
NE - Dia & 4 & 5 & 20 & 17,56 & 947,71 & 2,41 \\
NE - Noite & 4 & 6 & 40 & 13,64 & $2.948,19$ & 2,18 \\
\hline \hline
\end{tabular}

Conforme as Tabelas 4 e 5, os autores verificaram que o CTA/ha e o CA/ha aumentaram com a ETm, tanto na irrigação diurna quanto noturna. Verificaram também que as áreas irrigadas no horário noturno são inferiores àquelas do diumo devido a menor jornada de trabalho. 
Em relação ao CTA/ha, concluíram que a adoção da tarifação noturna para a irrigação se mostrou viável nas regiões $\mathrm{CO}$ e NE, onde houve redução de seu valor em $20 \%$ e $44 \%$. Já em relação ao $\mathrm{CA} / \mathrm{ha}$, constataram que em todas regiões seu valor foi maior devido à instalação da rede elétrica exclusiva até o conjunto moto-bomba e à aquisição do "REP".

Estudo semelhante ao realizado por Scaloppi (1985) fizeram Franke \& Dorfman (1997), ou seja, analisaram o custo de acionamento de conjuntos motobombas a partir de fontes de energia elétrica, fóssil e biomassa em um sistema de irrigação por aspersão tipo pivô central de 69 ha, na cultura do milho, para a condição edafoclimática do Planalto do Rio Grande do Sul. Verificaram que, para um determinado nível de manejo da irrigação, a energia elétrica consumida pela unidade de bombeamento foi $1369,21 \mathrm{kWh} / \mathrm{ha}$, a um custo de US\$ 68,31 por ha. Para o diesel o consumo foi $400,45 \mathrm{dm}^{3} / \mathrm{ha}$, a um custo de US\$ 142,96 por ha; para a gasolina foi $431,69 \mathrm{dm}^{3} / \mathrm{ha}$, a um custo de US $\$ 277,58$ por ha; e para o álcool foi $572,79 \mathrm{dm}^{3} /$ ha, a um custo de US\$331,65 por ha. Estes números indicaram custos de energia 109\%, 306\% e 386\% maiores para conjuntos moto-bombas acionados a óleo diesel, gasolina, e álcool, respectivamente, em relação ao elétrico. Estas porcentagens, segundo os autores, foram bem menores que as encontradas por Scaloppi (1985), visto que em 1985 a energia elétrica era fortemente subsidiada e os preços da energia fóssil e de biomassa eram bem mais altos do que os atualmente praticados.

\subsection{Diâmetro econômico para uma instalação elevatória}

"Dada uma vazão a transportar a determinada distância, por meio de bombeamento, por exemplo, a fixação do diâmetro e conseqüente perda de carga será orientada, em última análise, por considerações de ordem econômica. Os fatores que interessam são geralmente complexos: custo da primeira instalação, da exploração, etc. Em geral, cada caso concreto terá de ser estudado separadamente, por vezes com métodos próprios, como sucede nos grandes condutos de estações elevatórias e nos condutos de carga das centrais" (Lencastre, 1972).

"Na escolha do diâmetro mais econômico, tem-se que levar em consideração o custo da instalação, incluindo neste a aquisição, o transporte e a colocação do material, bem como, em alguns casos, a aquisição do terreno por onde vai passar o encanamento. Tal estudo é, de certo modo, complexo, exigindo inúmeros dados locais, além de dados de juros e capitalização, para depois ser resolvido com o auxílio dos problemas de máximos e mínimos" (Daker, 1987). 
“Teoricamente, o diâmetro de uma linha de recalque pode ser qualquer. Fazendo o recalque com velocidades de escoamento baixas, resultam diâmetros relativamente grandes, implicando em custos elevados da tubulação e menores gastos com as bombas e energia elétrica, porque as alturas manométricas são menores. Se, ao contrário, fazendo o recalque com velocidades altas, o diâmetro estabelecido será relativamente pequeno, resultando perdas elevadas. Como conseqüência, as alturas manométricas são maiores, os conjuntos mais potentes e mais caros, exigindo maior consumo de energia elétrica. Tecnicamente, entretanto, são feitas restrições quanto às velocidades mínimas (para evitar problemas de deposição) e às máximas (para evitar problemas de desgastes por abrasão). Entre os valores mínimos e máximos de velocidades há, em geral, diversos pares de diâmetro-potência que satisfazem os requisitos de demanda. A escolha do par mais adequado é feita através de considerações econômicas onde se procura o custo total mínimo, em termos de valor presente, levando em conta os custos decorrentes do investimento inicial e de operação e manutenção" (Coiado e Rivelli, 1993). A Figura 1 ilustra esta afirmativa.

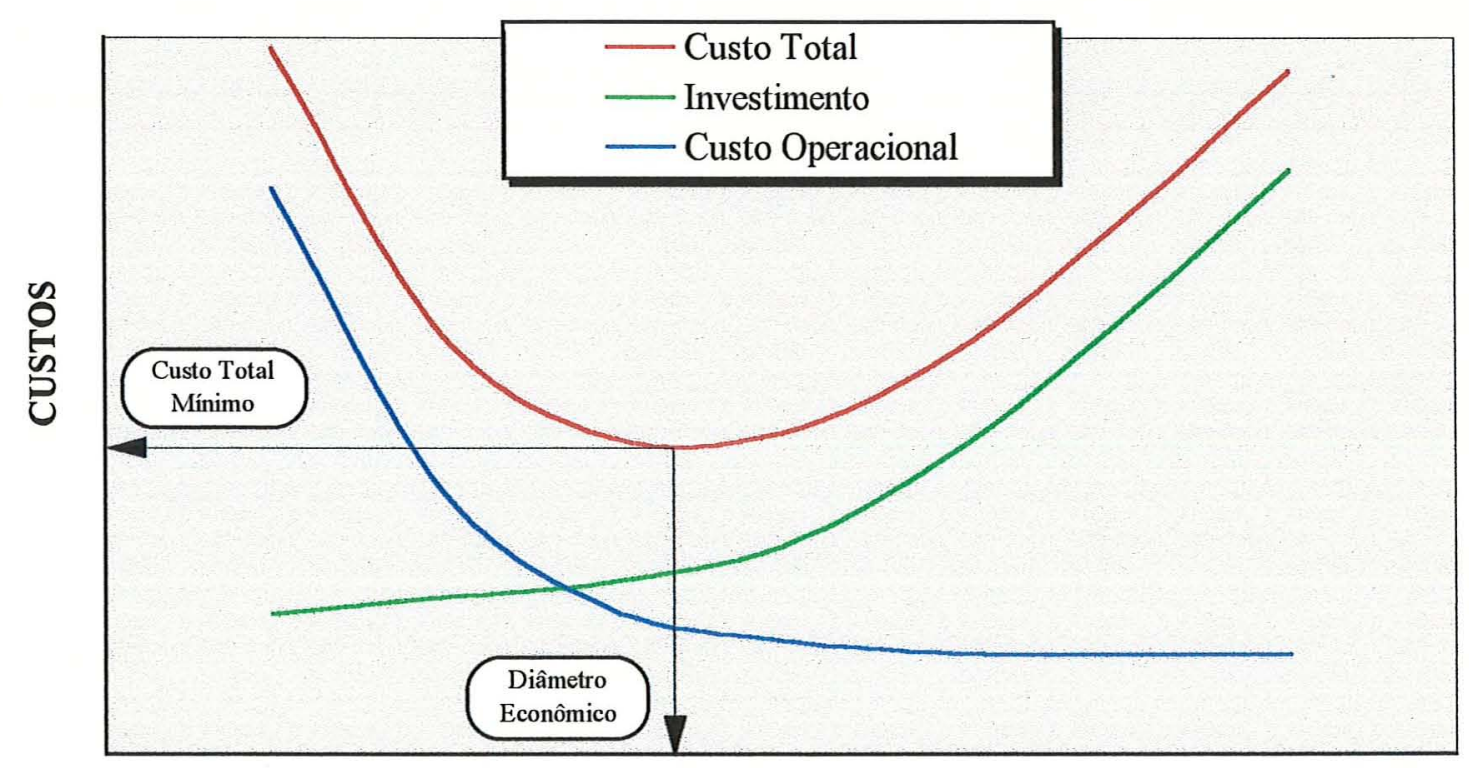

\section{DIÂMETRO}

Figura 1. Custo total, investimento e custo operacional em função do diâmetro da tubulação de um sistema de recalque.

Baseado nesse critério, chamado de Critério do Custo Total Mínimo, existem várias fórmulas que permitem o cálculo do diâmetro econômico para um sistema elevatório. Entre elas a 
de Bresse, que data do século passado, citado por Azevedo (1991). Em uma primeira aproximação são admitidos:

$\mathrm{p}_{1}$ : um preço médio por unidade de potência instalada para um conjunto elevatório, incluindo unidades de reserva, conservação e custeio capitalizado, em $\$$ por cavalo vapor;

$\mathrm{p}_{2}$ : um preço médio por unidade de comprimento de um conduto de diâmetro unitário assentado, em $\$ / m$.

O custo dos conjuntos elevatórios (\$) é:

$$
\mathrm{P}_{1}=\frac{\gamma \cdot \mathrm{Q} \cdot \mathrm{HM}}{75 \cdot \eta} \cdot \mathrm{p}_{1}
$$

em que: $\gamma$ - peso específico da água, normalmente considerado $1000 \mathrm{kgf} / \mathrm{m}^{3}$;

$\mathrm{Q}$ - vazão do sistema, em litros por segundo;

$\eta$ - rendimento do conjunto motor-bomba.

$\mathrm{HM}$ - altura manométrica do sistema, em metros, obtida por:

$$
\mathrm{HM}=\mathrm{H}+\frac{\mathrm{k} \cdot \mathrm{Q}^{2} \cdot \mathrm{L}}{\mathrm{D}^{5}}
$$

em que: $\mathrm{H}$ - altura total de recalque, em metros;

L - comprimento da tubulação, em metros;

D - diâmetro da tubulação, em metros;

$\mathrm{k}$ - coeficiente obtido de fórmulas práticas, sendo neste caso (fórmula universal):

$$
k=\frac{8}{\pi^{2} \cdot g} \cdot f
$$

em que: $f$ - fator de atrito da fórmula universal de perda de carga;

$\mathrm{g}$ - aceleração da gravidade terrestre $\left(9,806 \mathrm{~m} / \mathrm{s}^{2}\right)$.

O custo da tubulação (\$) é:

$$
P_{2}=\text { D.L. } p_{2}
$$

O custo total da instalação é, então:

$$
\mathrm{C}=\frac{\gamma \cdot \mathrm{Q} \cdot \mathrm{p}_{1}}{75 \cdot \eta} \cdot\left(\mathrm{H}+\frac{\mathrm{k} \cdot \mathrm{Q}^{2} \cdot \mathrm{L}}{\mathrm{D}^{5}}\right)+\mathrm{D} \cdot \mathrm{L} \cdot \mathrm{p}_{2}
$$

Para que o custo seja minimo, $\mathrm{dC} / \mathrm{dD}=0$, ou seja:

$$
\frac{d C}{d D}=\frac{-\gamma \cdot Q^{3} \cdot p_{1} \cdot k}{15 \cdot \eta \cdot D^{6}}+L \cdot p_{2}=0 \Rightarrow D=\sqrt[6]{\frac{k \cdot \gamma}{15 \cdot \eta}} \cdot \sqrt[6]{\frac{p_{1}}{p_{2}}} \cdot \sqrt{Q} \Rightarrow D=K \cdot \sqrt{Q}
$$


que é a conhecida fórmula de Bresse, aplicável a instalações de funcionamento contínuo. Verificase, portanto, que o funcionamento de uma linha de recalque é feito por imposições econômicas, o mesmo acontecendo com as linhas que alimentam as usinas hidrelétricas. Em fins do século passado, foi determinado um valor aproximado para $\mathrm{K}$, em função dos preços da época (1886):

$$
\left.\begin{array}{rl}
p_{1} & =4.000 \text { francos } \\
p_{2} & =100 \text { francos } \\
\eta & =0,60 \\
k & =0,00256
\end{array}\right\} \mathrm{K}=1,5 \Rightarrow \mathrm{V}=0,57 \mathrm{~m} / \mathrm{s}
$$

Para as condições brasileiras têm sido adotados valores para $K$ entre 0,9 e 1,4, sendo freqüente o uso do valor 1,2 , que resulta dos seguintes componentes:

$$
\left.\begin{array}{c}
\mathrm{p}_{1} / \mathrm{p}_{2}=9,0 \\
\eta=0,65 \\
\mathrm{k}=0,0032
\end{array}\right\} \mathrm{K}=1,2 \Rightarrow \mathrm{V}=0,88 \mathrm{~m} / \mathrm{s}
$$

Tratando-se de pequenas instalações, a fórmula de Bresse pode levar a um diâmetro aceitável. Para o caso de grandes instalações, dará uma primeira aproximação, sendo conveniente uma pesquisa econômica, em que sejam investigados os diâmetros mais próximos, inferiores e superiores. A velocidade nas canalizações de recalque, geralmente, é superior a $0,66 \mathrm{~m} / \mathrm{s}$, raramente ultrapassando $2,40 \mathrm{~m} / \mathrm{s}$. Esse limite superior é mais comumente encontrado nas instalações em que as bombas funcionam apenas algumas horas por dia.

Diante dos elevados custos da energia elétrica no Brasil, as adutoras que operam continuamente devem ser dimensionadas para uma velocidade média de escoamento de $1,0 \mathrm{~m} / \mathrm{s}$, relata Tsutiya (1989). Nos Estados Unidos e Portugal, a velocidade econômica também é da ordem de $1,0 \mathrm{~m} / \mathrm{s}$.

Quando o funcionamento da instalação de recalque não é contínuo, o diâmetro econômico pode ser calculado pela fórmula de Forccheimer ou pela fórmula da Associação Brasileira de Normas Técnicas - ABNT, citados por Neves (1982):

$$
\begin{gathered}
\mathrm{D}=\sqrt{\frac{4}{\pi} \cdot \frac{\sqrt{\mathrm{X}}}{0,6}} \cdot \sqrt{\mathrm{Q}}=1,46 \cdot \mathrm{X}^{0,25} \cdot \sqrt{\mathrm{Q}} \quad \text { (Forccheimer) } \\
\mathrm{D}=1,3 \cdot \mathrm{T}^{0,25} \cdot \sqrt{\mathrm{Q}} \quad(\mathrm{ABNT})
\end{gathered}
$$

sendo: D - diâmetro da tubulação, em m;

$\mathrm{Q}$ - vazão do sistema, em $\mathrm{m}^{3} / \mathrm{s}$; 
$\mathrm{X}$ - número de horas de trabalho da instalação por ano dividido por 8640 ;

$\mathrm{T}$ - número de horas de trabalho da instalação por dia dividido por 24 .

Camp (1952) estabeleceu a seguinte equação para calcular o custo anual da tubulação e da perda de carga por unidade de comprimento $(\mathrm{ft})$ de comprimento de uma adutora com bombeamento à vazão constante:

$$
A C=B \cdot r \cdot d+F \cdot a \cdot r \cdot d^{1,55}+236 \cdot p \cdot\left(\frac{1,594}{C}\right)^{1,85} \cdot \frac{q^{2,85}}{d^{4,87}}
$$

sendo: $\mathrm{r}$ - taxa anual de juros mais depreciação;

$\mathrm{d}$ - diâmetro da tubulação em polegadas (in);

F - fator relacionado ao tubo, tal como revestimento e presença de acessórios;

a - custo do tubo de ferro fundido com frete incluído, em centavos de dolar, por libra;

p - custo do bombeamento de 1 milhão de galões a altura de 1 pé (ft), em centavos de dolar;

q - vazão, em pés cúbicos por segundo;

C - coeficiente de rugosidade da fórmula de Hazen-Williams;

B - coeficiente relacionado ao custo do assentamento da tubulação, obtido por:

$$
\mathbf{B}=[(0,06+0,02 \cdot D) \cdot W+0,19 \cdot L+0,007 \cdot Y] \cdot d
$$

sendo: D - profundidade da vala, em pés;

W - preço da mão-de-obra comum, em centavos de dolar, por hora;

L - custo do chumbo, em centavos de dolar, por libra;

$\mathrm{Y}$ - custo da corda alcatroada (estopa), em centavos de dolar, por libra.

Quando a derivada primeira da equação (2.15) em relação ao diâmetro é igualada a zero, a seguinte relação é obtida para o diâmetro econômico:

$$
\text { B.r. } d^{5,87}+1,55 \cdot \text { F.a.r. } d^{6,42}=1150 \cdot p \cdot\left(\frac{1,594}{C}\right)^{1,85} q^{2,85}
$$

Uma solução aproximada da equação (2.17) é obtida, assumindo-se que o expoente do diâmetro em ambos os termos é 6,15 :

$$
d_{e c}=\frac{27,4}{C^{0,30}} \cdot\left[\frac{p}{r(B+1,55 \cdot F \cdot a)}\right]^{0,163} \cdot q^{0,46}
$$

O coeficiente 27,4 foi ajustado para compensar a alteração nos expoentes. 
Babbitt et al (1973) elaboraram equações muito semelhantes àquelas de Camp, obtendo velocidade econômica da ordem de $1,20 \mathrm{~m} / \mathrm{s}$ para diâmetros entre 100 e $900 \mathrm{~mm}$. Tanto Camp quanto Babbitt et al não consideram em seus trabalhos o custo do conjunto motor-bomba.

Cuomo \& Villela (1961) propuseram um procedimento de cálculo do diâmetro mais econômico em que consideram o custo do conduto $(\mathrm{Cc})$ e o custo das perdas no escoamento $(\mathrm{Cp})$.

O custo do conduto, em unidades monetárias - \$, é dado pela seguinte equação:

$$
\mathrm{Cc}=\mathrm{c} \cdot \mathrm{m} \cdot \mathrm{a} \cdot \mathrm{D}^{\nu} \cdot \ell
$$

sendo: c - custo unitário do tubo $(\$ / \mathrm{kg})$;

$\mathrm{m}$ - coeficiente que considera as juntas, custo de instalação e equipamentos;

D - o diâmetro do conduto $(\mathrm{m})$;

a e $v$ - coeficientes que variam com o material da tubulação e, para um o mesmo material, variam com o diâmetro, porém se mantêem constantes em largos intervalos deste último;

$\ell$ - comprimento da tubulação $(\mathrm{m})$;

Obs: o valor de $a . D^{v}$ dá o peso por metro da tubulação.

0 custo das perdas no escoamento, em $\$$, é dado pela equação:

$$
\mathrm{Cp}=\frac{\mathrm{ta} \cdot \mathrm{r}}{\mathrm{i}} \cdot\left[1-\frac{1}{(1+\mathrm{i})^{\mathrm{T}}}\right] \frac{9,8}{\eta} \cdot \mathrm{b} \cdot \mathrm{D}^{-\mu} \cdot \ell \cdot \mathrm{Q}^{3}
$$

sendo: ta - número de horas de funcionamento das bombas em 1 ano;

$r$ - custo da energia $(\$ / k W h)$;

i - taxa de juros anual;

T - tempo de amortização (anos);

$\eta$ - rendimento do grupo motor-bomba;

b e $\mu$-coeficientes hidráulicos da fórmula de Conti, obtidos através de tabelas;

$\mathrm{Q}$ - vazão do sistema $\left(\mathrm{m}^{3} / \mathrm{s}\right)$.

Os autores tornaram mínima a função $f=C c+C p$, fazendo-se $F=f / \ell$ e derivandose em relação a $\mathrm{D}$, obtendo-se:

$$
\frac{d F}{d D}=c \cdot m \cdot a \cdot v \cdot D^{v-1}-\mu \cdot \frac{\text { ta } \cdot r}{i} \cdot\left[1-\frac{1}{(1+i)^{T}}\right] \frac{9,8}{\eta} \cdot b \cdot D^{-(\mu+1)} \cdot Q^{3}=0 \Rightarrow
$$




$$
\mathrm{D}=\left\{\frac{\mu \cdot \mathrm{b}}{\mathrm{a} \cdot v} \cdot \frac{9,8 \cdot \mathrm{Q}^{3}}{\eta \cdot c \cdot \mathrm{m}} \cdot \frac{\mathrm{ta} \cdot \mathrm{r}}{\mathrm{i}} \cdot\left[1-\frac{1}{(1+\mathrm{i})^{\mathrm{T}}}\right]\right\}^{\frac{1}{v+\mu}}
$$

Como $\frac{d^{2} F}{d D^{2}}>0$, verifica-se que se trata de um mínimo.

A equação (2.21) foi aplicada para dimensionar uma adutora por recalque de fibrocimento; desnivel de $80 \mathrm{~m} ; \ell=2000 \mathrm{~m} ; \mathrm{Q}=0,1 \mathrm{~m}^{3} / \mathrm{s} ; \eta=0,7 ; \mathrm{c}=\operatorname{Cr} \$ 15,00 / \mathrm{kg} ; \mathrm{r}=1,50$ $\mathrm{Cr} \$ / \mathrm{kWh} ; \mathrm{i}=6 \% ; \mathrm{T}=20$ anos; $\mathrm{ta}=2920$ horas; $\mathrm{a}=684,0 ; v=2,00 ; \mathrm{b}=0,001 ; \mu=5,31 . \mathrm{O}$ valor do diâmetro obtido foi $0,3079 \mathrm{~m}$, sendo que o comercial adotado foi $300 \mathrm{~mm}$.

Deb (1978) desenvolveu um modelo matemático-econômico de um sistema de bombeamento, em que o custo total foi dividido em duas partes, ou seja, o custo do capital e o custo de operação e manutenção. Esse modelo é reproduzido a seguir.

O custo de uma tubulação instalada (\$/pé) pode ser expresso como uma função do diâmetro:

$$
\mathrm{y}_{1}=\mathrm{k}_{1} \cdot \mathrm{D}^{\mathrm{m}_{1}}
$$

sendo: D - diâmetro da tubulação, em polegadas (in);

$k_{1}$ e $m_{1}$ - coeficiente e expoente da função de custo;

O custo das bombas instaladas (\$) pode ser expressado como uma função da vazão e da altura manométrica total:

$$
\mathrm{y}_{2}=\mathrm{k}_{2} \cdot \mathrm{H}^{\mathrm{m}_{2}} \mathrm{Q}^{\mathrm{m}_{3}}
$$

sendo: $k_{2}, m_{2}$ e $m_{3}$ - coeficiente e expoentes da função;

$\mathrm{H}$ - altura manométrica total, em pés $(\mathrm{ft})$;

$\mathrm{Q}$ - vazão, em galões por minuto (gpm)

A potência das bombas (HP) pode ser expressada como:

$$
\mathrm{HP}=\frac{\mathrm{Q} \cdot\left(\mathrm{H}_{\mathrm{s}}+\mathrm{H}_{\mathrm{f}}\right) \cdot 8,33}{33000 \cdot \mathrm{E}_{\mathrm{p}}}
$$

sendo: $H_{s}$ - pressão estática, em pés (ft);

$\mathrm{H}_{\mathrm{f}}$ - perda de carga, em pés ( $\mathrm{ft}$ );

$E_{p}$ - eficiência das bombas.

A perda de carga pode ser dada pela equação de Hazen-Williams: 


$$
H_{f}=\frac{L \cdot Q^{p}}{0,0955 \cdot C^{p} \cdot D^{q}}
$$

sendo: $\mathrm{L}$ - comprimento da tubulação, em pés ( $\mathrm{ft}$ );

C - coeficiente de rugosidade de Hazen-Williams, sendo considerado o valor no final da vida útil;

p e q - expoentes da equação, cujos valores são 1,85 e 4,86, respectivamente.

Combinando-se as equações (2.23) e (2.25), o custo das bombas instaladas pode ser expressado por:

$$
\mathrm{y}_{2}=\mathrm{k}_{2} \cdot \mathrm{Q}^{\mathrm{m}_{3}} \cdot\left(\mathrm{H}_{\mathrm{s}}+\frac{\mathrm{L} \cdot \mathrm{Q}^{\mathrm{p}}}{0,0955 \cdot \mathrm{C}^{\mathrm{p}} \cdot \mathrm{D}^{\mathrm{q}}}\right)^{\mathrm{m}_{2}}
$$

O custo médio anual com energia e manutenção pode ser obtido pela equação:

$$
Y_{3}=\frac{(1+f) \cdot 54436 \cdot Q \cdot c}{33000 \cdot E_{m} \cdot E_{p}} \cdot\left(H_{s}+\frac{L \cdot Q^{p}}{0,0955 \cdot C^{p} \cdot D^{q}}\right)^{m_{2}}
$$

sendo: f - fração do custo da energia que é gasto com a manutenção do sistema, cujo valor sugerido por Linaweaver ${ }^{7}$ é 0,08 ;

c - custo unitário da energia $\$ / \mathrm{kWh}$;

$E_{m}$ - eficiência mecânica das bombas (relacionada às perdas na transmissão).

Para se obter o custo anual total do sistema, utiliza-se o fator de recuperação do capital, calculado pela expressão:

$$
R=\frac{i \cdot(1+i)^{n}}{(1+i)^{n}-1} \cdot(1-s)+i . s
$$

sendo: i - taxa de juros anual;

n - número de anos de vida útil dos equipamentos;

$\mathrm{s}$ - fração residual do custo dos equipamentos após a vida útil.

Combinando-se as equações (2.22) e (2.28), obtém-se o custo anual da tubulação (\$), ou seja:

$$
Y_{1}=y_{1} \cdot L \cdot R_{1}=k_{1} \cdot D^{m_{1}} \cdot L \cdot\left[\frac{i \cdot(1+i)^{n_{1}}}{(1+i)^{n_{1}}-1} \cdot(1-s)+i \cdot s\right]
$$

\footnotetext{
${ }^{7}$ LINAWEAVER Jr., P.F.; CLARK, C.S. Cost of water transmission. Journal of the american water works association, v.56, n.12, p.1549, Dec. 1964.
} 
Similarmente, o custo anual das bombas $(\$)$ é obtido combinando-se as equações $(2.23)$ e (2.28):

$$
Y_{2}=y_{2} \cdot R_{2}=k_{2} \cdot\left[\frac{i \cdot(1+i)^{n_{2}}}{(1+i)^{n_{2}}-1} \cdot(1-s)+i \cdot s\right] \cdot Q^{m_{3}} \cdot\left(H_{s}+\frac{L \cdot Q^{p}}{0,0955 \cdot C^{p} \cdot D^{q}}\right)^{m_{2}}
$$

O custo anual total do sistema é obtido somando-se as equações $(2.27),(2.29)$ e (2.30), ou seja:

$$
Y=R_{1} \cdot k_{1} \cdot D^{m_{2}} \cdot L+R_{2} \cdot k_{2} \cdot\left[Q^{m_{3}} \cdot\left(H_{s}+\frac{X \cdot L}{D^{q}}\right)^{m_{2}}\right]+k_{3} \cdot F \cdot\left(H_{s}+\frac{X \cdot L}{D^{q}}\right)
$$

em que: $F=\frac{Q}{33000 \cdot E_{p}} ; \quad X=\frac{Q^{p}}{0,0955 \cdot C^{p}} ;$ e $k_{3}=\frac{(1+f) 54436 \cdot c}{E_{m}}$

A equação (2.31) permite a obtenção do custo anual total em função do diâmetro da tubulação, para uma dada vazão $Q$, comprimento $L$ e pressão estática $H_{s}$. Para se obter o diâmetro econômico da tubulação, diferencia-se esta equação em relação ao diâmetro e iguala-se a zero, ou seja:

$$
\begin{gathered}
\frac{d Y}{d D}=R_{1} \cdot k_{1} \cdot L \cdot m_{1} \cdot D^{m_{1}-1}-R_{2} \cdot k_{2} \cdot m_{2} \cdot\left[Q^{m_{3}} \cdot\left(H_{s}+\frac{X \cdot L}{D^{q}}\right)^{m_{2}-1}\right] \cdot q \cdot X \cdot L \cdot D^{-(q+1)}+ \\
-q \cdot F \cdot k_{3} \cdot X \cdot L \cdot D^{-(q+1)}=0
\end{gathered}
$$

A solução da equação (2.32) somente é obtida por tentativa e erro. Pela análise de sensibilidade, todavia, verifica-se que o segundo termo do lado direito da equação não tem efeito significativo sobre o valor do diâmetro econômico, o que possibilita sua desconsideração, ficando:

$$
\frac{d Y}{d D}=R_{1} \cdot k_{1} \cdot L \cdot m_{1} \cdot D^{m_{1}-1}-q \cdot F \cdot k_{3} \cdot X \cdot L \cdot D^{-(q+1)}=0 \Rightarrow D=\left(\frac{q \cdot F \cdot k_{3} \cdot X}{R_{1} \cdot k_{1} \cdot m_{1}}\right)^{1 /\left(m_{1}+q\right)}
$$

Retornando-se os valores de $\mathrm{F}$ e $\mathrm{X}$ e expressando-se em relação à vazão $\mathrm{Q}$, a equação (2.33) fica:

$$
\mathbf{D}=K \cdot \mathbf{Q}^{\frac{p+1}{m_{1}+q}}
$$

em que: $K=\left(\frac{q}{R_{1} \cdot k_{1} \cdot m_{1}} \cdot \frac{1}{33000 \cdot E_{p}} \cdot \frac{k_{3}}{0,0955 \cdot C^{p}}\right)^{1 /\left(m_{1}+q\right)}$

O método desenvolvido por Deb foi aplicado a um sistema de bombeamento considerando-se os seguintes dados: $Q=1500 \mathrm{gpm} ; \mathrm{L}=6000 \mathrm{ft} ; \mathrm{H}_{\mathrm{s}}=160 \mathrm{ft} ; \mathrm{i}=0,06 ; \mathrm{n}_{1}=30 ; \mathrm{n}_{2}=15 ; \mathrm{s}_{1}$ 
$=0,1 ; s_{2}=0,2 ; C=100 ; p=1,85 ; q=4,86 ; E_{p}=0,8 ; E_{m}=0,8 ; c=0,03 \$ / k W h ; f=0,08 ; k_{1}=$ 1,$01 ; \mathrm{m}_{1}=1,29 ; \mathrm{m}_{2}=0,642 ; \mathrm{m}_{3}=0,453 ; \mathrm{k}_{2}=16,14$. O diâmetro econômico, obtido pela equação (2.34), foi 18,8 in. Também verificou-se que o mesmo diminuiu com o aumento da taxa de juros. Uma análise de sensibilidade do modelo em relação ao custo da energia foi realizado, sendo verificado que se se passar de $0,03 \$ / \mathrm{kWh}$ para $0,1 \$ / \mathrm{kWh}$, o diâmetro econômico passa de 13,8 in para 17,0 in. Assim, o custo da tubulação aumentaria com o aumento do custo da energia para se minimizar o custo total do sistema.

Lencastre (1983) considera pequena a influência da diferença de custos das estações elevatórias, em face dos custos da tubulação. Assim pode-se, pelo menos em primeira aproximação, considerar somente o investimento da tubulação. Considerando-se que o peso específico da água, $\gamma$, é $9800 \mathrm{~N} / \mathrm{m}^{3}$; e que o rendimento da bomba hidráulica, $\eta$, é 0,75 , a equação completa para se obter o diâmetro econômico, $\mathrm{D}$, em metros é:

$$
D=\left\{\frac{1}{(1+b)^{n}} \cdot \frac{1+b}{a-b} \cdot\left[1-\frac{1}{\left(1+\frac{a-b}{1+b}\right)}\right]\right\}^{\frac{3}{16+3 \cdot \alpha}}\left(\frac{260000}{\alpha \cdot C_{2} \cdot K_{s}^{2}}\right)^{\frac{3}{16+3 \cdot \alpha}} \cdot\left(\frac{C_{k} \cdot T_{f}}{1000}\right)^{\frac{3}{16+3 \cdot \alpha}} \cdot Q^{\frac{3}{16+3 \cdot \alpha}}
$$

sendo: $\alpha$ e $\mathrm{C}_{2}$ - coeficientes que se obtêm da análise de custo local das canalizações prontas, no qual se inclui o custo dos tubos, acessórios, montagem, abertura e fechamento de valas e todos os trabalhos acessórios que variem com o diâmetro;

$\mathrm{n}$ - vida útil da instalação, em anos;

a - taxa de juros anual;

b - taxa de acréscimo anual do volume de água aduzido;

$\mathrm{K}_{\mathrm{s}}$ - coeficiente de rugosidade da fórmula de Manning-Strickler;

$\mathrm{C}_{\mathrm{k}}$ - preço do $\mathrm{kWh}$;

$\mathrm{T}_{\mathrm{f}}$ - tempo de bombeamento diário no final da vida útil da instalação, em horas;

$\mathrm{Q}$ - vazão, $\mathrm{em} \mathrm{m}^{3} / \mathrm{s}$.

A equação (2.35) foi aplicada para dimensionar o diâmetro de uma adutora de concreto com: $\mathrm{Q}=0,350 \mathrm{~m}^{3} / \mathrm{s} ; \mathrm{n}=15$ anos; $\mathrm{b}=0,025 ; \mathrm{a}=0,12 ; \mathrm{C}_{\mathrm{k}}=3,00 \$ / \mathrm{kWh} ; \mathrm{T}_{\mathrm{f}}=24$ horas; $\mathrm{K}_{\mathrm{s}}$ $=75 ; \alpha=1,3$ e $C_{2}=17$. $O$ diâmetro encontrado foi de aproximadamente $600 \mathrm{~mm}$, o que resulta numa velocidade média de escoamento no primeiro ano de $1,24 \mathrm{~m} / \mathrm{s}$, e no décimo quinto ano de $1,79 \mathrm{~m} / \mathrm{s}$. 
Mais recentemente, Coiado \& Rivelli (1993) propuseram um modelo bastante detalhado para dimensionamento do diâmetro econômico de uma instalação de recalque, em que são considerados também a evolução dos custos da energia elétrica ao longo dos anos subsequentes ao ano de projeto. Uma reprodução dos principais pontos abordados é feita a seguir.

O custo total da instalação (\$) é dado pela equação:

$$
\mathrm{C}_{\mathrm{T}}=\mathrm{C}_{1}+\mathrm{C}_{2}+\mathrm{C}_{3}
$$

sendo: $\mathrm{C}_{1}$ - custo da tubulação (\$);

$\mathrm{C}_{2}$ - custo do conjunto motor-bomba (\$);

$\mathrm{C}_{3}$ - custo total da energia elétrica referente a $n$ anos.

O custo da tubulação pode ser determinado através da equação:

$$
\mathrm{C}_{1}=\gamma_{\mathrm{f}} \cdot \pi \cdot \mathrm{D} \cdot \mathrm{L}_{\mathrm{w}} \cdot \text { e. } \mathrm{c}_{1}
$$

em que: $\gamma_{\mathrm{f}}$ - peso especifico do material da tubulação $\left(\mathrm{kgf} / \mathrm{m}^{3}\right)$;

D - diâmetro da tubulação $(m)$;

$c_{1}$ - custo unitário da tubulação corrigido para o ano considerado $(\$ / \mathrm{kg})$;

$L_{\mathrm{w}}$ - comprimento virtual (m) equivalente ao peso da tubulação, obtido por:

$$
\mathrm{L}_{\mathrm{w}}=\mathrm{L}_{\mathrm{r}}+\mathrm{L}_{\mathrm{ew}}
$$

em que: $L_{r}$ - comprimento real da tubulação $(m)$;

$\mathrm{L}_{\mathrm{ew}}$ - comprimento da tubulação com peso equivalente ao peso da peças (m);

e - espessura das paredes do tubo (m), que para o ferro dúctil K9 ○ Catálogo Geral 77 da

Barbará indica a seguinte equação:

$$
\mathrm{e}=\frac{4,5+0,009 \cdot \mathrm{D}}{1000}
$$

O custo de cada conjunto motor-bomba da instalação foi estimado com base no custo médio da sua unidade de potência $(\$ / \mathrm{cv})$, considerando uma folga de potência do motor elétrico de $15 \%$ em relação à bomba:

$$
\mathrm{C}_{2}=\frac{\gamma \cdot \mathrm{Q}_{1}}{75 \cdot \eta_{\mathrm{c}}} \cdot\left[\mathrm{H}_{\mathrm{g}}+\frac{10,65 \cdot \mathrm{Q}_{2}^{1,85}}{\mathrm{C}^{1,85} \cdot \mathrm{D}^{4,87}} \cdot\left(\mathrm{L}_{\mathrm{r}}+\mathrm{L}_{\mathrm{e}}\right)\right] 1,15 \cdot \mathrm{c}_{2}
$$

sendo: $\gamma$ - peso específico da água, considerado $1000 \mathrm{kgf} / \mathrm{m}^{3}$;

$\mathrm{Q}_{1}$ - vazão da bomba $\left(\mathrm{m}^{3} / \mathrm{s}\right)$;

$\eta_{\mathrm{c}}$ - rendimento do conjunto motor-bomba;

$\mathrm{H}_{\mathrm{g}}$ - altura geométrica total $(\mathrm{m})$; 
$\mathrm{Q}_{2}$ - vazão da adutora $\left(\mathrm{m}^{3} / \mathrm{s}\right)$;

C - coeficiente de rugosidade da equação de Hazen-Williams;

$\mathrm{L}_{e}$ - comprimento equivalente às perdas localizadas $(\mathrm{m})$;

$\mathrm{c}_{2}$ - custo da unidade de potência do conjunto motor-bomba corrigido para o ano considerado $(\$ / c v)$.

O custo da energia elétrica foi calculado considerando-se o funcionamento ininterrupto do sistema e a evolução dos preços das tarifas de energia elétrica referente ao Grupo A - Modalidade Convencional, tomando-se por base o índice de variação percentual acumulado de tais tarifas:

$$
\mathrm{C}_{3}=\frac{0,009676 \cdot \gamma \cdot \mathrm{Q}_{1}}{\eta_{\mathrm{c}}} \cdot\left[\mathrm{H}_{\mathrm{g}}+\frac{10,65 \cdot \mathrm{Q}_{2}^{1,85}}{\mathrm{C}^{1,85} \cdot \mathrm{D}^{4,87}} \cdot\left(\mathrm{L}_{\mathrm{r}}+\mathrm{L}_{\mathrm{e}}\right)\right] \sum_{\mathrm{i}=1}^{\mathrm{n}}\left(\mathrm{Td}_{\mathrm{i}}+0,72 \cdot \mathrm{Tc}_{\mathrm{i}}\right) \cdot 12
$$

sendo: $\mathrm{Td}_{\mathrm{i}}$ - tarifa de demanda corrigida do i-ésimo ano dentre os $n$ anos subsequentes ao do projeto, em que se considera a evolução dos custos da energia elétrica ( $\$ / \mathrm{kW}$;

$\mathrm{Tc}_{\mathrm{i}}$ - tarifa de consumo corrigida do i-ésimo ano dentre os $n$ anos subsequentes ao do projeto, em que se considera a evolução dos custos da energia elétrica $(\$ / 1000 . \mathrm{kWh})$.

Substituindo-se as equações (2.38) e (2.39) na equação (2.37), bem como o parâmetro $Q_{1}$ por $Q_{2} / 2$ nas equações $(2.40)$ e (2.41), e derivando-se o custo total em relação ao diâmetro da tubulação, tem-se:

$$
\frac{\mathrm{dC}_{\mathrm{T}}}{\mathrm{dD}}=\gamma_{\mathrm{f}} \cdot\left(\mathrm{L}_{\mathrm{r}}+\mathrm{L}_{\mathrm{ew}}\right) \cdot \mathrm{c}_{1} \cdot\left(\frac{141+0,565 \cdot \mathrm{D}}{10000}\right)-\psi
$$

em que: $\quad \psi=\frac{\gamma \cdot \mathrm{Q}_{2}^{2,85} \cdot\left(\mathrm{L}_{\mathrm{r}}+\mathrm{L}_{\mathrm{e}}\right)}{\eta_{\mathrm{c}} \cdot \mathrm{C}^{1,85} \cdot \mathrm{D}^{5,87}} \cdot\left[0,398 \cdot \mathrm{c}_{1}+\sum_{\mathrm{i}=1}^{\mathrm{n}}\left(\mathrm{Td}_{\mathrm{i}}+0,72 \cdot \mathrm{Tc}_{\mathrm{i}}\right) \cdot 12\right]$

Fazendo-se $\mathrm{dC}_{\mathrm{T}} / \mathrm{dD}=0$, tem-se o ponto de custo mínimo, com $\mathrm{D}=\mathrm{D}_{\mathrm{e}}$ (diâmetro econômico), que isolado na equação torna-se:

$$
D_{e}=\left\{\frac{\frac{\gamma \cdot\left(L_{r}+L_{e}\right)}{\eta_{\mathrm{c}} \cdot C^{1,85}} \cdot\left[0,398 \cdot c_{2}+0,251 \cdot \sum_{i=1}^{\mathrm{n}}\left(\mathrm{Td}_{\mathrm{i}}+0,72 \cdot \mathrm{Tc}_{\mathrm{i}}\right) \cdot 12\right]}{0,0142 \cdot \gamma_{\mathrm{f}} \cdot\left(\mathrm{L}_{\mathrm{r}}+\mathrm{L}_{\mathrm{ew}}\right) \cdot \mathrm{c}_{1}}\right]^{0,17} \cdot \mathrm{Q}^{0,486}
$$

A equação (2.43) foi aplicada ao novo sistema de recalque de Capivari, que recalca água bruta do Rio Capivari até a ETA, e que apresenta os seguintes dados técnicos: $\mathrm{H}_{\mathrm{g}}=21 \mathrm{~m} ; \mathrm{L}_{\mathrm{r}}$ $=154 \mathrm{~m} ; \mathrm{L}_{\mathrm{e}}=45,5 \mathrm{~m} ; \mathrm{Q}_{1}=0,211 \mathrm{~m}^{3} / \mathrm{s} ; \mathrm{Q}_{2}=0,422 \mathrm{~m}^{3} / \mathrm{s} ; \eta_{\mathrm{c}}=0,75 ; \mathrm{C}=97 ; \mathrm{D}_{\mathrm{a}}$ (diâmetro atual) $=$ 
$0,500 \mathrm{~m}$; tubulação de ferro dúctil $\mathrm{K} 9 ; \gamma_{\mathrm{f}} \mathrm{L}_{\mathrm{w}}=1,434 \times 10^{6} \mathrm{kgf} . \mathrm{m} / \mathrm{m}^{3}$; casa de bombas com três conjuntos motor-bombas associados em paralelo, de potência de $100 \mathrm{cv}$ cada, sendo que dois operam continuamente e um permanece como reserva. Os preços de referência levantados em julho de 1992 foram: $c_{1}=11.325,00 \mathrm{Cr} \$ / \mathrm{kg} ; c_{2}=325.000,00 \mathrm{Cr} \$ / \mathrm{cv} ; \mathrm{Td}=13.929,85 \mathrm{Cr} \$ / \mathrm{kW} ; \mathrm{Tc}=$ $203.925,92 \mathrm{Cr} \$ / 1000 \mathrm{kWh}$. O número de anos subsequentes ao do projeto, $n$, em que se considera a evolução dos custos da energia elétrica foi igual a 5 .

Os resultados das aplicações da equação (2.43) mostraram que o $\mathrm{D}_{\mathrm{e}}$ cresceu exponencialmente do ano de 1980 para o ano 1991 , ou seja, de $0,832 \mathrm{~m}$ para $8,169 \mathrm{~m}$, respectivamente. Com isso, os autores concluíram que, dependendo do valor $n$, o diâmetro assumirá valores muito grandes. Neste caso, o $\mathrm{D}_{\mathrm{e}}$ deverá respeitar o menor valor da velocidade média de escoamento permitido sem que haja depósito de materiais transportado em suspensão, no caso de bombeamento de água bruta.

O modelo proposto por Coiado \& Rivelli (1993), embora seja bastante detalhado por envolver muitos parâmetros, apresenta resultados discutíveis em função dos seguintes aspectos:

i) não é considerado nos $n$ anos subsequentes ao do projeto a amortização e a remuneração do custo unitário da tubulação $\left(\mathrm{c}_{1}\right)$ e do custo da unidade de potência do conjunto motor-bomba $\left(c_{2}\right)$, ou seja, considera-se somente o somatório das tarifas de energia elétrica corrigidas (por índice especifico). Com isso, se o índice de correção das tarifas for elevado neste período, o efeito do custo da energia será extremamente intenso sobre os demais, mesmo que se considere um período curto (por exemplo $\mathrm{n}=2$ ). Portanto, para se corrigir tal distorção, seria necessário também acrescentar às equações (2.37) e (2.40) a amortização e a remuneração dos custos unitários corrigidos (por índices específicos) $c_{1}$ e $c_{2}$ dos $n$ anos subsequentes ao do projeto.

ii) o valor de $n$ não está associado ao número de anos de vida útil dos equipamentos, fato que deveria ocorrer para que o efeito do custo da energia elétrica pudesse ser adequadamente representado no custo total do sistema;

iii) da mesma maneira que as outras fórmulas apresentadas neste item, excetuando-se a fórmula de Deb (1978), a relação existente entre a potência do motor-bomba e seu custo não é linear na maioria das vezes, pois a tendência é que à medida que a potência aumente reduz-se o custo unitário. Sendo assim, seria mais econômico a aquisição de um conjunto motor-bomba do que dois de metade da potência cada um. 


\section{METODOLOGIA}

Um sistema de recalque de água possui, basicamente, os seguintes componentes:

i) Elétricos (quando a bomba hidráulica é acionada por motor elétrico):

- linha de alta tensão (que vai da linha-tronco até a estação de bombeamento);

- transformador;

- motor elétrico;

- comando de partida;

- acessórios (cabos elétricos para baixa tensão, isoladores, conduítes e demais acessórios necessários para interligar o transformador, comando de partida e motor elétrico).

Caso o acionamento da bomba hidráulica seja por motor à combustão e, mais especificamente, a óleo diesel, considera-se o equipamento completo (motor, bomba injetora, tanque de combustivel, bateria, comando de partida, sistema de refrigeração e todos acessórios necessários para seu funcionamento).

ii) Hidráulicos:

- tubos;

- bomba hidráulica;

- acessórios (válvula de pé, curvas, redução excêntrica, ampliação concêntrica, válvula de retenção, registro de gaveta, manômetro e outros mais);

iii) Obras civis:

- casa de bombas;

- valas para assentamento da tubulação adutora (quando for o caso);

Com isso, o custo anual total do sistema pode ser obtido pela seguinte equação:

$$
\mathrm{CATS}=\mathrm{CFAS}+\mathrm{CVAS}
$$

sendo: CATS - custo anual total do sistema (\$);

CFAS - custo fixo anual do sistema (\$);

CVAS - custo variável anual do sistema (\$). 


\subsection{Custo fixo anual total do sistema}

O custo fixo anual do sistema pode ser obtido pela equação:

$$
\text { CFAS }=\left[\frac{\text { CSN } \cdot(1-R) \cdot r}{(1+r)^{\mathrm{PA}}-1}+\frac{\mathrm{CSN} \cdot(1+\mathrm{R}) \cdot \mathrm{r}}{2}\right]
$$

sendo: CSN - custo do sistema novo (\$);

R - fração do custo do sistema novo após o periodo de depreciação. É de dificil previsão, porém está diretamente relacionado com o desgaste fisico e avarias, devido à intensidade de uso do sistema, e com a obsolescência tecnológica do mesmo.

$\mathrm{r}$ - taxa de juros anual;

PA - período de depreciação do sistema (anos).

O primeiro membro da equação diz respeito ao valor anual de reposição e o segundo da remuneração do capital investido.

O custo do sistema novo, para conhecimento do investimento inicial, é obtido por:

$$
\mathrm{CSN}=\mathrm{CCH}+\mathrm{COCC}+\mathrm{CLEE} \text { ou CMCA }
$$

sendo: $\mathrm{CCH}$ - custo dos componentes hidráulicos (\$);

COCC - custo das obras de construção civil para o sistema (\$);

CLEE - custo da linha e equipamentos elétricos (\$) para bomba hidráulica acionada por motor elétrico;

CMCA - custo do motor à combustão e acessórios (\$) para bomba hidráulica acionada por motor à combustão (diesel);

\subsubsection{Metodologia para estimativa do custo do equipamento novo}

Verifica-se que existe uma relação funcional tipo multiplicativa entre o diâmetro ou potência do equipamento hidráulico e elétrico novo e seu custo, conforme Zocoler (1994) ou seja:

$$
C E Q_{i z}=a_{i} \cdot Z^{b_{i}}
$$

sendo: $\mathrm{CEQ}_{\mathrm{iZ}}$ - custo (\$) do i-ésimo equipamento novo no diâmetro ou potência $Z$;

$a_{i}$ e $b_{i}$ - coeficientes de ajuste da regressão do i-ésimo equipamento;

$Z$ - diâmetro (m) ou potência (W) do i-ésimo equipamento. 
Sendo assim, todos equipamentos utilizados nos sistemas de recalque tiveram sua função de custo ajustada desta forma, permitindo que a estimativa do CSN fosse possivel, pois mesmo que não exista comercialmente o diâmetro ou potência de um determinado equipamento, estima-se seu custo por tal função.

No item 4 podem ser observados os valores dos coeficientes $a_{i}$ e $b_{i}$ dos equipamentos utilizados na aplicação, bem como as principais informações das estatisticas das regressões.

\subsubsection{Custo dos componentes hidráulicos}

O custo dos componentes hidráulicos do sistema é obtido por:

$$
\mathrm{CCH}=\mathrm{CTs}+\mathrm{CTr}+\mathrm{CBH}
$$

sendo: CTs - custo da tubulação de sucção (\$), obtido pela equação:

$$
C T s=\frac{L s}{L u} \cdot a_{T s} \cdot\left(\frac{4 \cdot Q}{\pi \cdot V s}\right)^{\frac{b_{T s}}{2}}+\sum_{j=1}^{J} n_{j} \cdot a_{j} \cdot\left(\frac{4 \cdot Q}{\pi \cdot V s}\right)^{\frac{b_{j}}{2}}
$$

sendo: Ls - comprimento da tubulação de sucção (m), em que são desconsiderados os comprimentos dos acessórios;

$\mathrm{Lu}$ - comprimento de um tubo (m);

$a_{\mathrm{Ts}}, b_{\mathrm{Ts}}, a_{\mathrm{j}}$ e $b_{j}$ - coeficientes de ajuste da regressão para o tubo de sucção e para o jésimo acessório da sucção;

$\mathrm{n}_{\mathrm{j}}$ - número de unidades do j-ésimo acessório da sucção;

$\mathrm{Q}$ - vazão do sistema adutor $\left(\mathrm{m}^{3} / \mathrm{s}\right)$;

Vs - velocidade de escoamento na sucção $(\mathrm{m} / \mathrm{s})$, que deve ser em média igual a 1,0 $\mathrm{m} / \mathrm{s}$ e não superior a $1,5 \mathrm{~m} / \mathrm{s}$, conforme Carvalho (1989). Também não deve ser inferior a 0,45 m/s (evitar deposição de partículas), segundo Martins (1966);

CTr - custo da tubulação de recalque (\$), obtido pela equação:

$$
\mathrm{CTr}=\frac{\mathrm{Lr}}{\mathrm{Lu}} \cdot \mathrm{a}_{\mathrm{Tr}} \cdot \phi^{\mathrm{b}_{\mathrm{Tr}}}+\sum_{\ell=1}^{\mathrm{L}} \mathrm{n}_{\ell} \cdot \mathrm{a}_{\ell} \cdot \phi^{\mathrm{b}_{\ell}}
$$

sendo: $\mathrm{Lr}$ - comprimento da tubulação de recalque (m), em que são desprezados os comprimentos dos acessórios;

$a_{T_{r}}, b_{T r}, a_{\ell}$ e $b_{\ell}$-coeficientes de ajuste da regressão para o tubo de recalque e para o $\ell$-ésimo acessório do recalque; 
$\mathrm{n}_{\ell}$ - número de unidades do l-ésimo acessório do recalque;

$\phi$ - diâmetro da tubulação de recalque (m);

$\mathrm{CBH}$ - custo da bomba hidráulica (\$), obtido pela equação:

$$
\mathrm{CBH}=\mathrm{a}_{\mathrm{BH}} \cdot(\mathrm{Q} \cdot \mathrm{HM} \cdot \gamma)^{\mathrm{b}_{\mathrm{BH}}}
$$

sendo: $a_{\mathrm{BH}}$ e $\mathrm{b}_{\mathrm{BH}}$ - coeficientes de ajuste da regressão para a bomba hidráulica;

$\gamma$ - peso especifico da água, assumido como $9806 \mathrm{~N} / \mathrm{m}^{3}$;

$\mathrm{HM}$ - altura manométrica do sistema (m), obtida por:

$$
\mathrm{HM}=\mathrm{HR}+\mathrm{PF}+\mathrm{HFs}+\mathrm{HFr}
$$

sendo: HR - altura de recalque $(\mathrm{m})$;

PF - carga manométrica necessária no final da adutora $(\mathrm{m})$;

HFs - perda de carga na sucção (m), obtida por:

$$
\mathrm{HFs}=5,912 \cdot \frac{\mathrm{Vs}^{2,436} \cdot \mathrm{Ls}}{\mathrm{Q}^{0,584} \cdot \mathrm{Cs}^{1,852}}+\sum_{\mathrm{j}=1}^{\mathrm{J}} \mathbf{n}_{\mathrm{j}} \cdot \mathrm{K}_{\mathrm{j}} \cdot \frac{\mathrm{Vs}^{2}}{2 \cdot \mathrm{g}}
$$

sendo: Cs - coeficiente relacionado à rugosidade dos tubos da sucção;

$\mathrm{K}_{\mathrm{j}}$ - coeficiente de perda de carga localizada do j-ésimo acessório da sucção;

$\mathrm{g}$ - aceleração da gravidade, considerada $9,806 \mathrm{~m} / \mathrm{s}^{2}$.

$\mathrm{HFr}$ - perda de carga no recalque (m), obtida por:

$$
\mathrm{HFr}=10,649 \cdot \frac{\mathrm{Q}^{1,852} \cdot \mathrm{Lr}}{\mathrm{Cr}^{1,852} \cdot \phi^{4,871}}+\sum_{\ell=1}^{\mathrm{L}} \mathrm{n}_{\ell} \cdot \mathrm{K}_{\ell} \cdot \frac{8 \cdot \mathrm{Q}^{2}}{\mathrm{~g} \cdot \pi \cdot \phi^{4}}
$$

sendo: $\mathrm{Cr}$ - coeficiente relacionado à rugosidade dos tubos do recalque;

$\mathrm{K}_{\ell}$ - coeficiente de perda de carga localizada do $\ell$-ésimo acessório do recalque.

Fazendo-se as substituições, a equação (3.5) torna-se:

$$
\mathrm{CCH}=\frac{\mathrm{Ls}}{\mathrm{Lu}} \cdot \mathrm{a}_{\mathrm{Ts}} \cdot\left(\frac{4 \cdot \mathrm{Q}}{\pi \cdot \mathrm{Vs}}\right)^{\frac{\mathrm{b}_{\mathrm{BS}}}{2}}+\sum_{\mathrm{j}=1}^{J} \mathrm{n}_{\mathrm{j}} \cdot \mathrm{a}_{\mathrm{j}} \cdot\left(\frac{4 \cdot \mathrm{Q}}{\pi \cdot \mathrm{Vs}}\right)^{\frac{\mathrm{b}_{\mathrm{j}}}{2}}+\frac{\mathrm{Lr}}{\mathrm{Lu}} \cdot \mathrm{a}_{\mathrm{Tr}} \cdot \phi^{\mathrm{b}_{\mathrm{TI}}}+\sum_{\ell=1}^{\mathrm{L}} \mathrm{n}_{\ell} \cdot \mathrm{a}_{\ell} \cdot \phi^{\mathrm{b}_{\ell}}+\mathrm{a}_{\mathrm{HH}} \cdot(\mathrm{Q} \cdot \gamma \cdot \mathrm{HM})^{\mathrm{b}_{\mathrm{PH}}}
$$

sendo que:

$$
\mathrm{HM}=\mathrm{HR}+\mathrm{PF}+5,912 \cdot \frac{\mathrm{Vs}^{2,436} \cdot \mathrm{Ls}}{\mathrm{Q}^{0,584} \cdot \mathrm{Cs}^{1,852}}+\sum_{\mathrm{j}=1}^{j} \mathrm{n}_{\mathrm{j}} \cdot \mathrm{K}_{\mathrm{j}} \cdot \frac{\mathrm{Vs}^{2}}{2 \cdot \mathrm{g}}+10,649 \cdot \frac{\mathrm{Q}^{1,852} \cdot \mathrm{Lr}}{\mathrm{Cr}^{1,852} \cdot \phi^{4,871}}+\sum_{\ell=1}^{\mathrm{L}} \mathrm{n}_{\ell} \cdot \mathrm{K}_{\ell} \cdot \frac{8 \cdot \mathrm{Q}^{2}}{\mathrm{~g} \cdot \pi \cdot \phi^{4}}
$$




\subsubsection{Custo da linha de energia e equipamentos elétricos}

O custo da linha e equipamentos elétricos de um sistema de recalque é obtido por:

$$
\mathrm{CLEE}=\mathrm{CLAT}+\mathrm{CEEBT}
$$

sendo: CLAT - custo da linha de alta tensão (\$), obtido por:

$$
\text { CLAT }=\text { LLAT . CMLAT }
$$

sendo: LLAT - comprimento da linha de alta tensão (m), ou seja, distância entre a estação de bombeamento e a linha-tronco de alta tensão no ponto de derivação;

CMLAT - custo médio da linha de alta tensão $(\$ / m)$. O custo médio da linha de alta tensão é influenciado, basicamente, pela topografia local, tipos de obstáculos presentes no trajeto (divisas principais, cercas, locais de seccionamento e aterramento, travessias, matas, várzeas e outros mais) e pela carga por ela atendida. Os primeiros (topografia e obstáculos) influenciam principalmente na quantidade de postes, isoladores, cruzetas, grampos, elos fusíveis, pára-raios e outros mais, enquanto o último influencia principalmente na seção dos cabos condutores, muito embora para as distâncias e cargas encontradas normalmente em sistemas de recalque de propriedades rurais esta influência possa ser desprezada (Exemplo: um ramal trifásico de $13,8 \mathrm{kV}$ de tensão, com cabos CAA - 4 AWG $\left(21,2 \mathrm{~mm}^{2}\right)$, que é freqüentemente utilizado, pode alimentar uma carga de $500 \mathrm{~kW}$ a uma distância de aproximadamente $14 \mathrm{~km}$, apresentando queda de tensão de $5 \%$, que está no limite admitido para este fim, segundo Piedade Júnior (1983).

CEEBT - custo dos equipamentos elétricos de baixa tensão (\$), que pode ser obtido por:

$$
\mathrm{CEEBT}=\mathrm{CME}+\mathrm{CTE}+\mathrm{CCP}+\mathrm{CBC}+\mathrm{CAE}
$$

sendo: CME - custo do motor elétrico (\$), estimado pela equação:

$$
\mathrm{CME}=\mathrm{a}_{\mathrm{ME}} \cdot\left(\frac{1,1 \cdot \mathrm{Q} \cdot \mathrm{HM} \cdot \gamma}{\eta_{\mathrm{BH}}}\right)^{\mathrm{b}_{\mathrm{MF}}}
$$

sendo: $a_{M E}$ e $b_{M E}$ - coeficientes de ajuste da regressão multiplicativa de custo do motor elétrico em função de sua potência nominal em watt, que é obtida pelo termo entre parêntesis;

$\eta_{B H}$ - rendimento da bomba hidráulica; 
Obs: o coeficiente 1,1 refere-se à reserva de potência do motor acionador da bomba hidráulica (elétrico ou diesel).

CTE - custo do transformador $(\$)$, estimado pela equação:

$$
\mathrm{CTE}=a_{\mathrm{TE}} \cdot\left(\frac{1,1 \cdot \mathrm{Q} \cdot \mathrm{HM} \cdot \gamma}{\eta_{\mathrm{ME}} \cdot \cos \varphi \cdot \eta_{\mathrm{BH}}}\right)^{b_{\mathrm{TR}}}
$$

sendo: $a_{\mathrm{TE}}$ e $b_{\mathrm{TE}}$ - coeficientes de ajuste da regressão para o transformador ;

$\eta_{\text {ME }}$ - rendimento do motor elétrico;

$\cos \varphi$ - fator de potência nominal do motor elétrico;

CCP - custo do comando de partida (\$), estimado pela equação:

$$
\mathrm{CCP}=\mathrm{a}_{\mathrm{CP}} \cdot\left(\frac{1,1 \cdot \mathrm{Q} \cdot \mathrm{HM} \cdot \gamma}{\eta_{\mathrm{BH}}}\right)^{\mathrm{b}_{\mathrm{CP}}}
$$

sendo: $a_{C P}$ e $b_{C P}$ - coeficientes de ajuste da regressão para o comando de partida;

CBC - custo do banco de capacitores (\$), utilizado para corrigir, quando necessário, o fator de potência do circuito (motor elétrico) para 0,92, que é o mínimo admitido pela concessionária de energia elétrica para isenção do ajuste referente ao fator de potência na tarifa de energia elétrica, podendo ser estimado por:

$$
\mathrm{CBC}=\mathrm{a}_{\mathrm{BC}} \cdot\left\{[\operatorname{tg}(\arccos \varphi)-\operatorname{tg}(\operatorname{arc} \cos 0,92)] \frac{1,1 \cdot \mathrm{Q} \cdot \mathrm{HM} \cdot \gamma}{\eta_{\mathrm{BH}}}\right\}^{\mathrm{b}_{\mathrm{CP}}}
$$

sendo: $a_{B C}$ e $b_{B C}$ - coeficientes de ajuste da regressão para o banco de capacitores;

$\operatorname{arc} \cos \varphi-\operatorname{arco}(\mathrm{rad})$ correspondente à defasagem entre corrente e tensão no circuito elétrico do sistema sem o banco de capacitores;

$\operatorname{arc} \cos 0,92$ - $\operatorname{arco}(\mathrm{rad})$ correspondente à defasagem entre corrente e tensão no circuito elétrico do sistema com o banco de capacitores, cujo valor é 0,40271584 radianos;

Obs: o termo entre chaves corresponde à potência reativa corrigida pelo banco de capacitores;

CAE - custo dos acessórios elétricos (isoladores, conectores, cabos, eletrodutos, hastes de aterramento e outros mais) (\$), que embora varie bastante de uma situação para outra, pode ser estimado por: 


$$
\mathrm{CAE}=f_{\mathrm{TE}} . \mathrm{CTE}
$$

sendo: $f_{\mathrm{TE}}$ - fração do custo do transformador elétrico novo, considerado 0,45 , conforme Revolti ${ }^{2}$ (1996);

Fazendo-se as substituições, a equação (3.13) torna-se:

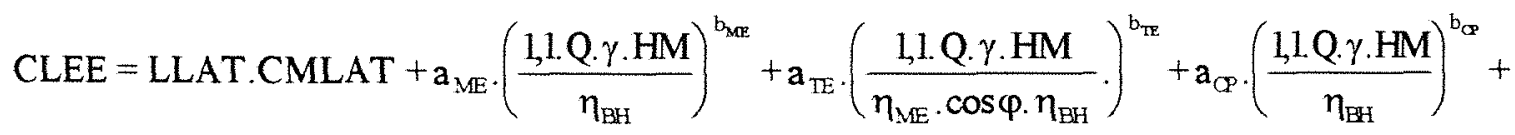

$$
\begin{aligned}
& a_{\mathrm{BC}} \cdot\left\{\frac{1,1 \cdot[\operatorname{tg}(\operatorname{arc} \cos \varphi)-0,402716] \cdot Q \cdot \gamma \cdot \mathrm{HM}}{\eta_{\mathrm{BH}}}\right\}^{b_{\mathrm{BC}}}+\mathrm{f}_{\mathrm{TE}} \cdot \mathrm{a}_{\mathrm{TE}} \cdot\left(\frac{1,1 \cdot \mathrm{Q} \cdot \gamma \cdot \mathrm{HM}}{\eta_{\mathrm{ME}} \cdot \cos \varphi \cdot \eta_{\mathrm{BH}}}\right)^{b_{\mathrm{JP}}}
\end{aligned}
$$

\subsubsection{Custo do motor à combustão e acessórios}

Pode ser estimado pela equação:

$$
\mathrm{CMCA}=\mathrm{a}_{\mathrm{MCA}} \cdot\left(\frac{1,1 \cdot \mathrm{Q} \cdot \mathrm{HM} \cdot \gamma}{\eta_{\mathrm{BH}}}\right)^{\mathrm{b}_{\mathrm{MCA}}}
$$

sendo: $\mathrm{a}_{\mathrm{MCA}}$ e $\mathrm{b}_{\mathrm{MCA}}$ - coeficientes de ajuste da regressão para o motor à combustão e acessórios.

\subsubsection{Custo das obras de construção civil}

O custo das obras de construção civil pode ser obtido por:

$$
\mathrm{COCC}=\mathrm{CCB}+\mathrm{CVT}
$$

sendo: CCB - custo da casa de bombas (\$), que varia muito com o porte, qualidade dos materiais de construção utilizados e condições do local a ser construida, porém pode ser estimada por:

$$
\mathrm{CCB}=\mathrm{CCC} . \mathrm{ACB}
$$

sendo: CCC - custo da construção civil para obras de acabamento rústico $\left(\$ / \mathrm{m}^{2}\right)$;

$\mathrm{ACB}$ - área da casa de bombas $\left(\mathrm{m}^{2}\right)$.

CVT - custo da vala para assentamento da adutora (\$), obtido por:

$$
\text { CVT }=\text { L. DV . WV . CVE }
$$

\footnotetext{
${ }^{2}$ REVOLTI, J. (Elétrica Re-Voltis, Jaboticabal, SP) Comunicação pessoal, 1996.
} 
sendo: DV - profundidade da vala $(\mathrm{m})$;

WV - largura da vala $(\mathrm{m})$;

CVE - custo da escavação - abertura, apiloamento e fechamento - $\left(\$ / \mathrm{m}^{3}\right)$.

Obs: assumiu-se que a adutora ficará enterrada (totalmente ou parcialmente).

Portanto, substituindo-se as equações (3.24) e (3.25) na (3.23), obtém-se:

$$
\mathrm{COCC}=\mathrm{CCC} \cdot \mathrm{ACB}+\mathrm{L} \cdot \mathrm{DV} \cdot \mathrm{WV} \cdot \mathrm{CVE}
$$

\subsection{Custo variável anual do sistema}

O custo variável anual do sistema pode ser obtido por:

$$
\mathrm{CVAS}=\mathrm{CABO}+\mathrm{CAMR}
$$

sendo: CABO - custo anual de bombeamento (\$);

CAMR - custo anual com manutenção e reparos (\$), que para o sistema com bomba hidráulica acionada por motor à combustão e para o sistema com bomba hidráulica acionada por motor elétrico são obtidos, respectivamente, por:

$$
\begin{aligned}
& \mathrm{CAMR}=\left[\mathrm{f}_{\mathrm{TAH}} \cdot(\mathrm{CTs}+\mathrm{CTr})+\mathrm{f}_{\mathrm{BH}} \cdot \mathrm{CBH}+\mathrm{f}_{\mathrm{MCA}} \cdot \mathrm{CMCA}+\mathrm{f}_{\mathrm{OCC}} \cdot \mathrm{COCC}^{*}\right] \cdot \frac{\mathrm{TFa}}{2000} \\
& \mathrm{CAMR}=\left[\mathrm{f}_{\mathrm{TAH}} \cdot(\mathrm{CTs}+\mathrm{CTr})+\mathrm{f}_{\mathrm{BH}} \cdot \mathrm{CBH}+\mathrm{f}_{\mathrm{ME}} \cdot \mathrm{CME}+\mathrm{f}_{\mathrm{EE}} \cdot \mathrm{CEE}+\right. \\
& \left.f_{L A T} \cdot C L A T+f_{O C C} \cdot C O C C^{*}\right) \cdot \frac{T F a}{2000}
\end{aligned}
$$

sendo: $f_{\mathrm{TAH}}$ - fração do custo da tubulação e acessórios novos gasto anualmente com manutenção e reparos para 2000 horas de operação, considerado 0,005 , conforme o PRONI (1987);

$f_{\mathrm{BH}}$ - fração do custo da bomba hidráulica nova gasto anualmente com manutenção $\mathrm{e}$ reparos para 2000 horas de operação, considerado 0,04 - PRONI (1987);

$\mathrm{f}_{\mathrm{MCA}}$ - fração do custo do motor à combustão e acessórios novos gasto anualmente com manutenção e reparos para 2000 horas de operação, considerado 0,065 - PRONI (1987) ;

$f_{O C C}$ - fração do custo das obras de construção civil gasto anualmente com manutenção e reparos para 2000 horas de operação, considerado 0,01 - PRONI (1987); 
$f_{\mathrm{ME}}$ - fração do custo do motor elétrico novo gasto anualmente com manutenção e reparos para 2000 horas de operação, considerado 0,02 - PRONI (1987);

$f_{E E}$ - fração do custo dos equipamentos elétricos (exceto o motor elétrico) e acessórios novos gasto anualmente com manutenção e reparos, considerado 0,005 (Revolti, 1996);

$f_{\text {LAT }}$ - fração do custo da linha de alta tensão gasto anualmente com manutenção e reparos, considerado 0,005 para 2000 horas de operação (Revolti, 1996);

$\mathrm{TFa}$ - tempo de funcionamento anual do sistema de recalque (h);

$\mathrm{COCC}^{*}$ - custo das obras de construção civil (\$), exclusive a vala para assentamento da adutora (abertura e fechamento);

\subsubsection{Custo anual de bombeamento para bombas acionadas por motor à combustão}

É obtido pela equação:

$$
\mathrm{CABO}=\mathrm{CC}_{\mathrm{u}} \cdot \mathrm{C}_{\mathrm{u}} \mathrm{C} \cdot \mathrm{TFa} \cdot\left(\frac{\mathrm{Q} \cdot \mathrm{HM} \cdot \gamma}{\eta_{\mathrm{BH}}}\right)
$$

sendo: $\mathrm{CC}_{\mathrm{u}}$ - consumo médio de combustivel (diesel) do motor por unidade de potência nominal por tempo de operação $\left(\mathrm{m}^{3} / \mathrm{W} . \mathrm{h}\right)$;

$\mathrm{C}_{\mathrm{u}} \mathrm{C}$ - custo da unidade de combustivel $\left(\$ / \mathrm{m}^{3}\right)$;

\subsubsection{Custo anual de bombeamento para bombas acionadas por motor elétrico}

É obtido pela equação adaptada do CODI (1988):

$$
\mathrm{CABO}=\mathrm{FD}+\mathrm{FC}+\mathrm{AJ}
$$

sendo: FD - faturamento anual da demanda (\$);

FC - faturamento anual do consumo $(\$)$;

$\mathrm{AJ}$ - ajuste anual referente ao fator de potência (\$).

Embora se tenha previsto a aquisição de um banco de capacitores para o sistema a fim de se obter isenção do ajuste anual referente ao fator de potência $(\mathrm{AJ})$, considerou-se o referido termo nas equações das modalidades de aplicação da tarifa de energia elétrica, pois assim pode-se utilizá-las também em sistemas que não possuem tal dispositivo. 


\subsubsection{Modalidades de aplicação das tarifas de energia elétrica}

O custo anual de bombeamento do sistema depende da modalidade de tarifação que o mesmo se enquadra diante da concessionária de energia elétrica, ou seja, da tarifa Convencional ou das Horo-Sazonais (verde e azul) que são tarifas de energia elétrica com custos diferenciados de acordo com sua utilização durante as horas do dia e durante os períodos do ano. Esta diferenciação de custos visa reduzir os custos de fornecimento da energia entregue ao consumidor, decorrente da otimização do sistema elétrico nacional. Também permitem ao consumidor reduzir suas despesas com energia elétrica desde que consiga programar o seu uso, ou seja, evitando-se o horário de ponta e/ou deslocando-se o consumo para determinados meses do ano. O horário de ponta corresponde a três horas consecutivas (definidas pela concessionária) entre as 17 e $22 \mathrm{~h}$ de segunda a sexta-feira, enquanto o horário fora de ponta são as horas complementares às de ponta, acrescidas à totalidade das horas dos sábados e domingos. O período seco é composto de sete meses consecutivos de maio a novembro (214 dias), enquanto o período úmido é composto de cinco meses consecutivos de dezembro a abril (151 dias).

As tarifas Horo-Sazonais e Convencional são aplicadas aos consumidores atendidos em tensão de fornecimento igual ou superior a $2,3 \mathrm{kV}$ (Grupo A) ou ligados em baixa tensão em sistema de distribuição subterrâneo, mas considerados para efeito de faturamento como de alta tensão. Para os consumidores do Subgrupo A4, no qual incluem-se os rurais, também são concedidos descontos especiais para os irrigantes que solicitarem tal beneficio, conforme a Portaria $\mathrm{n}^{\mathrm{0}} 105$ de 3 de abril de 1992 do DNAEE. Porém, tais descontos incidem somente sobre o consumo de energia elétrica entre as 23 e $5 \mathrm{~h}$, sendo necessário exclusividade para irrigação, ou seja, a rede elétrica para o sistema de irrigação deve ser independente das demais da propriedade, além do que os equipamentos de medição e controle da energia formecida ficam a cargo do consumidor. Os descontos variam conforme a região do país, sendo igual a $90 \%$ para o Nordeste e regiões geoeconômicas denominadas Vale do Jequitinhonha e Polígono da Seca, no Estado de Minas Gerais; $80 \%$ para o Norte e Centro-Oeste e demais regiões do Estado de Minas Gerais; e 70\% para as demais regiões do país.

As equações adaptadas do FD, FC e AJ, desenvolvidas a partir da equação (3.31), em cada modalidade de tarifação para um ano normal, ou seja, ano modelo para planejamento, são apresentados nos itens $3.2 .2 .2,3.2 .2 .3$ e 3.2 .2 .4 . 


\subsubsection{Tarifa convencional}

É aplicada às unidades consumidoras do Grupo A, atendidas em tensão inferior a 69 $\mathrm{kV}$ e com demanda menor do que $500 \mathrm{~kW}$.

\subsubsection{Faturamento da demanda:}

$$
F D=(12-d) \cdot D M \cdot T D c+0,10 \cdot d \cdot D M \cdot T D c
$$

sendo: DM - demanda medida $(\mathrm{kW})$, obtida pela equação:

$$
\mathrm{DM}=\frac{\mathrm{Q} \cdot \mathrm{HM} \cdot \gamma}{1000 \cdot \eta_{\mathrm{ME}} \cdot \eta_{\mathrm{BH}}}
$$

TDc - tarifa de demanda convencional $(\$ / \mathrm{kW})$;

$\mathrm{d}$ - número de meses completos por ano que o sistema de recalque fica desligado e, com isso, ocorre faturamento de demanda correspondente a $10 \%$ da maior demanda medida nos últimos 11 meses, ou seja a própria DM, pois o motor e equipamentos elétricos são exclusivos para acionamento da bomba hidráulica. Portanto, verifica-se que a equação (3.32) somente se aplica se $d \leq 11$.

\subsubsection{Faturamento do consumo}

a) sem o benefício da Portaria 105 do DNAEE:

$$
\mathrm{FC}=\mathrm{TC} . \mathrm{CMa}
$$

sendo: TCc - tarifa de consumo convencional $(\$ / \mathrm{kWh})$;

$\mathrm{CMa}$ - consumo anual medido $(\mathrm{kWh})$, obtido pela equação:

$$
\mathrm{CMa}=\left(\frac{\mathrm{Q} \cdot \mathrm{HM} \cdot \gamma}{1000 \cdot \eta_{\mathrm{ME}} \cdot \eta_{\mathrm{BH}}}\right) \cdot \mathrm{TFd} \cdot \mathrm{NDa}
$$

sendo: TFd - tempo médio de funcionamento diário do sistema de recalque $(h)$;

$\mathrm{NDa}$ - número de dias por ano de funcionamento do sistema de recalque.

b) com o benefício da Portaria 105 do DNAEE:

$$
\mathrm{FC}=\mathrm{TCc} .(\mathrm{CMa}-\mathrm{CMahe} . \mathrm{fdtc})
$$

sendo: fdtc - fração de desconto sobre a tarifa de consumo; 
CMahe - consumo anual medido $(\mathrm{kWh})$ no horário especial para irrigantes (23 as $5 \mathrm{~h})$, obtido por:

$$
\text { CMahe }=\left(\frac{Q \cdot H M \cdot \gamma}{1000 \cdot \eta_{\mathrm{ME}} \cdot \eta_{\mathrm{BH}}}\right) \cdot \text { TFdhe } \cdot \mathrm{NDa}
$$

sendo: TFdhe - tempo médio de funcionamento diário do sistema de recalque no horário especial para irrigantes.

\subsection{Ajuste do fator de potência}

a) sem o benefício da Portaria 105 do DNAEE:

$$
\mathrm{AJ}=[(12-\mathrm{d}) \cdot \mathrm{TDc} \cdot \mathrm{DM}+(\mathrm{TCc} \cdot \mathrm{CMa})] \cdot\left(\frac{0,92}{\cos \varphi}-1\right)
$$

sendo: $\cos \varphi$ - fator de potência medido, que neste caso corresponde aproximadamente ao fator de potência nominal do motor elétrico.

A equação (3.38) é aplicada somente quando $\cos \varphi \leq 0,92$.

b) com o benefício da Portaria 105 do DNAEE:

$$
\mathrm{AJ}=\{(12-\mathrm{d}) \cdot \mathrm{TDc} \cdot \mathrm{DM}+[\mathrm{TCc} \cdot(\mathrm{CMa}-\mathrm{CMahe} \cdot \mathrm{fdtc})]\} \cdot\left(\frac{0,92}{\cos \varphi}-1\right)
$$

A equação (3.39) é aplicada somente quando $\cos \varphi \leq 0,92$.

\subsubsection{Tarifa verde}

É aplicada sempre em caráter opcional às unidades consumidoras do Grupo A atendidas em tensão inferior a $69 \mathrm{kV}$ e com demanda de potência igual ou superior a $50 \mathrm{~kW}$.

\subsection{Faturamento da demanda}

$$
F D=(12-d) \cdot[D C \cdot T D v+(D M-D C) \cdot T U v]+0,10 \cdot d \cdot D M \cdot T D v
$$

sendo: DC - demanda contratada com a Concessionária de energia elétrica $(\mathrm{kW})$;

TDv - tarifa de demanda verde $(\$ / \mathrm{kW})$;

TUv - tarifa de ultrapassagem de demanda verde $(\$ / \mathrm{kW})$, que somente é aplicada se: $i)$ a demanda medida for superior a $10 \%$ da demanda contratada, quando a demanda contratada for superior a $100 \mathrm{~kW}$; ii) a demanda medida for superior a $20 \%$ da demanda contratada, quando a demanda contratada for de $50 \mathrm{~kW}$ a $100 \mathrm{~kW}$; 
Uma vez que os equipamentos elétricos são exclusivos para o motor que aciona a bomba hidráulica, a DC é igual a DM. Sendo assim, não há aplicação da tarifa de ultrapassagem de demanda e a equação (3.40) torna-se:

$$
F D=(12-d) \cdot D M \cdot T D v+0,10 \cdot d \cdot D M \cdot T D v
$$

A equação (3.41) somente se aplica se $\mathrm{d} \leq 11$.

\subsection{Faturamento do consumo}

a) sem o benefício da Portaria 105 do DNAEE:

$$
\text { FC }=\text { CMup. TCvup }+ \text { CMufp. TCvufp }+ \text { CMsp. TCvsp + CMsfp. TCvsfp }
$$

sendo: CMup - consumo anual medido $(\mathrm{kWh})$ no período úmido no horário de ponta (17 as $21 \mathrm{~h}$ ou definido pela concessionária), obtido por:

$$
\mathrm{CMup}=\left(\frac{\mathrm{Q} \cdot \mathrm{HM} \cdot \gamma}{1000 \cdot \eta_{\mathrm{ME}} \cdot \eta_{\mathrm{BH}}}\right) \cdot \operatorname{TFdp} \cdot \mathrm{NDu} \cdot \frac{5}{7}
$$

sendo: TFdp - tempo médio de funcionamento diário do sistema de recalque no horário de ponta (h);

NDu - número de dias de funcionamento do sistema de recalque no período úmido do ano.

CMufp - consumo anual medido $(\mathrm{kWh})$ no período úmido no horário fora de ponta (horas complementares à de ponta), obtido por:

$$
\text { CMufp }=\left(\frac{Q \cdot H M \cdot \gamma}{1000 \cdot \eta_{\mathrm{ME}} \cdot \eta_{\mathrm{BH}}}\right) \cdot\left(\operatorname{TFdfp}+\text { TFdp } \cdot \frac{2}{7}\right) \cdot \mathrm{NDu}
$$

sendo: TFdfp - tempo médio de funcionamento diário do sistema de recalque no horário fora de ponta $(\mathrm{h})$.

CMsp - consumo anual medido $(\mathrm{kWh})$ no período seco no horário de ponta, obtido por:

$$
\mathrm{CMsp}=\left(\frac{\mathrm{Q} \cdot \mathrm{HM} \cdot \gamma}{1000 \cdot \eta_{\mathrm{ME}} \cdot \eta_{\mathrm{BH}}}\right) \cdot \operatorname{TFdp} . \text { NDs. } \frac{5}{7}
$$

sendo: NDs - número de dias de funcionamento do sistema de recalque no período seco do ano.

CMsfp - consumo anual medido $(\mathrm{kWh})$ no período seco no horário fora de ponta, obtido por: 


$$
\mathrm{CMsfp}=\left(\frac{\mathrm{Q} \cdot \mathrm{HM} \cdot \gamma}{1000 \cdot \eta_{\mathrm{ME}} \cdot \eta_{\mathrm{BH}}}\right) \cdot\left(\mathrm{TFdfp}+\mathrm{TFdp} \cdot \frac{2}{7}\right) \cdot \mathrm{NDs}
$$

TCvsp - tarifa de consumo verde no periodo seco no horário de ponta $(\$ / \mathrm{kWh})$;

TCvsfp - tarifa de consumo verde no período seco no horário fora de ponta $(\$ / \mathrm{kWh})$;

TCvup - tarifa de consumo verde no periodo úmido no horário de ponta $(\$ / \mathrm{kWh})$;

TCvufp - tarifa de consumo verde no período úmido no horário fora de ponta $(\$ / \mathrm{kWh})$.

b) com o benefício da Portaria 105 do DNAEE:

$$
\begin{aligned}
\text { FC }= & \text { CMup. TCvup }+(\text { CMufp }- \text { CMuhe } . \text { fdtc }) \cdot \text { TCvufp }+ \\
& \text { CMsp. TCvsp }+(\text { CMsfp }- \text { CMshe } . \text { fdtc }) \cdot \text { TCvsfp }
\end{aligned}
$$

sendo: CMuhe - consumo anual medido $(\mathrm{kWh})$ no periodo úmido no horário especial para irrigantes, obtido por:

$$
\text { CMuhe }=\left(\frac{Q \cdot H M \cdot \gamma}{1000 \cdot \eta_{\mathrm{ME}} \cdot \eta_{\mathrm{BH}}}\right) \cdot \text { TFdhe.NDu }
$$

CMshe - consumo anual medido $(\mathrm{kWh})$ no periodo seco no horário especial para irrigantes, obtido por:

$$
\text { CMshe }=\left(\frac{\text { Q.HM. } \gamma}{1000 \cdot \eta_{\mathrm{ME}} \cdot \eta_{\mathrm{BH}}}\right) \cdot \text { TFdhe. NDs }
$$

\subsubsection{Ajuste do fator de potência}

a) sem o beneficio da Portaria 105 do DNAEE:

$$
\begin{gathered}
\mathrm{AJ}=[(12-\mathrm{d}) \cdot \mathrm{DM} \cdot \mathrm{TDv}+ \\
\text { CMup. TCvup + CMufp. TCvufp + CMsp. TCvsp + CMsfp. TCvsfp }] \cdot\left(\frac{0,92}{\cos \varphi}-1\right)
\end{gathered}
$$

A equação (3.50) é aplicada somente quando $\cos \varphi \leq 0,92$

b) com o benefício da Portaria 105 do DNAEE:

$$
\begin{gathered}
\mathrm{AJ}=[(12-\mathrm{d}) \cdot \mathrm{DM} \cdot \mathrm{TDv}+\text { CMup. TCvup }+(\text { CMufp }- \text { CMuhe } \text { fdtc }) \cdot \text { TCvufp }+ \\
\text { CMsp. TCvsp }+(\text { CMsfp }- \text { CMshe } \cdot \text { fdtc }) \cdot \text { TCvsfp }] \cdot\left(\frac{092}{\cos \varphi}-1\right)
\end{gathered}
$$

A equação (3.51) é aplicada somente quando $\cos \varphi \leq 0,92$. 


\subsubsection{Tarifa azul}

É aplicada compulsoriamente às unidades consumidoras do Grupo A atendidas em: i) tensão igual ou superior a $69 \mathrm{kV}$; ii) tensão inferior a $69 \mathrm{kV}$, com demanda de potência igual ou superior a $500 \mathrm{~kW}$, desde que não façam opção pela tarifa verde. Também é aplicada em caráter opcional às unidades consumidoras do Grupo A atendidas em tensão inferior a $69 \mathrm{kV}$ com demanda de potência entre $50 \mathrm{~kW}$ e $500 \mathrm{~kW}$.

\subsection{Faturamento da demanda}

$$
\begin{aligned}
& \text { FD }=(5-\text { dup }) \cdot[\text { DCup. TDap }+(\text { DM }- \text { DCup }) \cdot \text { TUap }]+0,10 \cdot \text { dup. DM.TDap }+ \\
& \text { (5-dufp).[DCufp .TDafp + (DM - DCufp).TUafp]+0,10.dufp. DM.TDafp + } \\
& (7-d s p) \cdot[D C s p \cdot \text { TDap + (DM - DCsp) TUap]+ 0,10.dsp. DM.TDap + } \\
& (7-d s f p) \cdot[\text { DCsfp .TDafp }+(\text { DM - DCsfp }) \cdot \text { TUafp }]+0,10 \cdot d s f p . D M . T D a f p
\end{aligned}
$$

sendo: DCup - demanda contratada no período úmido no horário de ponta $(\mathrm{kW})$;

DCufp - demanda contratada no periodo úmido no horário fora de ponta $(\mathrm{kW})$, que não poderá ser inferior a DCup;

DCsp - demanda contratada no periodo seco no horário de ponta $(\mathrm{kW})$, que não poderá ser superior a DCup;

DCsfp - demanda contratada no periodo seco no horário fora de ponta $(\mathrm{kW})$, que não poderá ser inferior a DCsp, e também não poderá ser superior a DCufp;

TDap - tarifa de demanda azul no horário de ponta $(\$ / \mathrm{kW})$;

TDafp - tarifa de demanda azul no horário fora de ponta $(\$ / \mathrm{kW})$;

TUap - tarifa de ultrapassagem de demanda azul no horário de ponta $(\$ / \mathrm{kW})$;

TUafp - tarifa de ultrapassagem de demanda azul no horário fora de ponta $(\$ / \mathrm{kW})$;

dup - número de meses completos que o sistema de recalque fica desligado no período úmido

do ano no horário de ponta of com isso, ocorre faturamento de demanda correspondente a $10 \%$ da maior demanda medida nos últimos 11 meses neste segmento (horário de ponta), ou seja DM. Porém, se a DCup for igual a zero, tal faturamento não ocorre;

dufp - número de meses completos que o sistema de recalque fica desligado no período úmido do ano no horário fora de ponta e, com isso, ocorre faturamento de demanda correspondente a $10 \%$ da maior demanda medida nos últimos 11 meses neste segmento (horário fora de ponta), ou seja DM; 
dsp - número de meses completos que o sistema de recalque fica desligado no periodo seco do ano no horário de ponta e, com isso, ocorre faturamento de demanda correspondente a $10 \%$ da maior demanda medida nos últimos 11 meses neste segmento (horário de ponta), ou seja DM. Porém, se a DCsp for igual a zero, tal faturamento não ocorre;

dsfp - número de meses completos que o sistema de recalque fica desligado no período seco do ano no horário fora de ponta e, com isso, ocorre faturamento de demanda correspondente a $10 \%$ da maior demanda medida nos últimos 11 meses neste segmento (horário fora de ponta), ou seja DM. Porém, se a DCsfp for igual a zero, tal faturamento não ocorre;

A tarifa de ultrapassagem de demanda azul tanto no horário de ponta quanto no horário fora de ponta será aplicada somente se: $i$ ) a demanda medida em um ou ambos casos for superior a $10 \%$ da demanda contratada para o segmento fora de ponta, quando a demanda contratada no respectivo segmento for superior a $100 \mathrm{~kW}$; ii) a demanda medida em um ou ambos casos for superior a $20 \%$ da demanda contratada para o segmento fora de ponta, quando a demanda contratada no respectivo segmento for de $50 \mathrm{~kW}$ a $100 \mathrm{~kW}$.

Como procedimento geral, é recomendada a contratação de demanda somente no(s) segmento(s) horo-sazonal(is) que o sistema tenha sido projetado para operar, embora esta contratação deva satisfazer as restrições já descritas que: DCup < DCufp $>$ DCsfp $>$ DCsp. Caso o mesmo venha a ser operado em segmento horo-sazonal não contratado, mesmo que esporadicamente, o faturamento da demanda será calculado pela ultrapassagem de demanda, que apresenta tarifa expressivamente superior (aproximadamente o triplo). Considerando a primeira afirmativa e sabendo-se que os equipamentos elétricos do sistema são exclusivos para o motor que aciona a bomba hidráulica, a DCup, DCufp, DCsp e DCsfp são iguais a DM. Com isso, na equação (3.52) não há aplicação da tarifa de ultrapassagem de demanda, tornando-se:

$$
\begin{aligned}
\text { FD }= & (5-\text { dup }) \cdot \text { DM. TDap + 0,10.dup. DM. TDap }+ \\
& (5-\text { dufp }) . \text { DM.TDafp + 0,10.dufp.DM.TDafp }+ \\
& (7-\text { dsp }) \cdot \text { DM.TDap + 0,10.dsp. DM.TDap }+ \\
& (7-\text { dsfp }) \cdot \text { DM.TDafp + 0,10.dsfp. DM.TDafp }
\end{aligned}
$$

Em conformidade com o último parágrafo, as restrições à aplicação da equação (3.53) são as seguintes:

- Se dufp = 5, então a equação (3.53) não se aplica; 
- Se dup $=5$, então os termos “0,10.dup.DM.TDap" e

"(7 - dsp). DM.TDap + 0,10.dsp. DM. TDap " não se aplicam;

- Se dsfp $=7$, então os termos "0,10.dsfp.DM.TDafp"

“(7-dsp).DM.TDap + 0,10.dsp. DM.TDap " não se aplicam;

- Se dsp $=7$, então o termo “0,10.dsp.DM.TDap" não se aplica.

\subsubsection{Faturamento do consumo}

a) sem o benefício da Portaria 105 do DNAEE:

$$
\text { FC }=\text { CMup. TCaup + CMufp. TCaufp }+ \text { CMsp. TCasp + CMsfp. TCasfp }
$$

sendo: TCaup - tarifa de consumo azul no período úmido no horário de ponta $(\$ / \mathrm{kWh})$;

TCaufp - tarifa de consumo azul no periodo úmido no horário fora de ponta $(\$ / \mathrm{kWh})$;

TCasp - tarifa de consumo azul no período seco no horário de ponta $(\$ / \mathrm{kWh})$;

TCasfp - tarifa de consumo azul no periodo seco no horário fora de ponta $(\$ / \mathrm{kWh})$.

b) com o benefício da Portaria 105 do DNAEE:

$$
\begin{aligned}
\mathrm{FC}= & \text { CMup. TCaup }+(\text { CMufp }- \text { CMuhe } . \text { fdtc }) \cdot \text { TCaufp }+ \\
& \text { CMsp. TCasp }+(\text { CMsfp }- \text { CMshe. fdtc }) \cdot \text { TCasfp }
\end{aligned}
$$

\subsection{Ajuste do fator de potência}

\section{a) sem o benefício da Portaria 105 do DNAEE:}

$\mathrm{AJ}=[(5-$ dup $) \cdot$ DM $\cdot$ TDap $+(5-$ dufp $) \cdot$ DM. TDafp $+(7-$ dsp $) \cdot$ DM.TDap + (7-dsfp). DM.TDafp + CMup. TCaup + CMufp.TCaufp + CMsp. TCasp + CMsfp. TCasfp] $\left[\left(\frac{0,92}{\cos \varphi}-1\right)\right.$

A equação (3.56) é aplicada somente quando $\cos \varphi \leq 0,92$.

b) com o benefício da Portaria 105 do DNAEE:

$\mathrm{AJ}=[(5-$ dup $) \cdot$ DM.TDap $+(5-$ dufp $) \cdot$ DM.TDafp $+(7-$ dsp $)$. DM.TDap $+(7-$ dsfp $)$. DM.TDafp + CMup. TCaup + (CMufp - CMuhe. fdtc) . TCaufp +

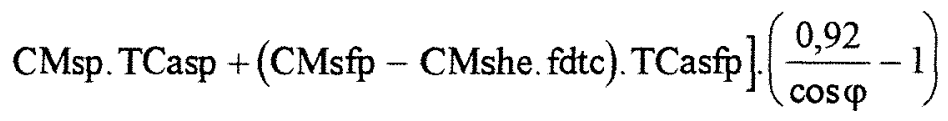

A equação (3.57) é aplicada somente quando $\cos \varphi \leq 0,92$. 


\subsection{Estimativa do custo anual total do sistema}

Uma vez que todos os parâmetros do CFAS e do CVAS que não possuiam valor fixo foram definidos em função do diâmetro da tubulação de recalque da adutora, pode-se reescrever a equação (3.1) direcionada para o tipo de acionamento da bomba hidráulica, diesel ou elétrico, e dentro do elétrico para as várias modalidades de tarifação da energia elétrica. Com isso, pode-se estimar o custo anual total do sistema substituindo-se na equação (3.1) o valor deste parâmetro, selecionando-se em seguida o de menor valor.

\subsubsection{Sistema com bomba hidráulica acionada por motor à combustão (D)}

Fazendo-se todas substituições, a equação (3.1) torna-se:

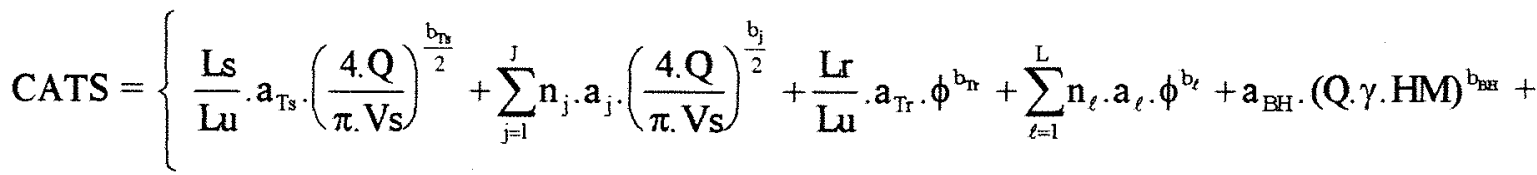

$$
\begin{aligned}
& \left.\mathrm{a}_{\mathrm{MCA}} \cdot\left(\frac{1,1 \cdot \mathrm{Q} \cdot \mathrm{HM} \cdot \gamma}{\eta_{\mathrm{BH}}}\right)^{\mathrm{b}_{\mathrm{MCA}}}+\mathrm{CCC} \cdot \mathrm{ACB}\right\} \cdot \frac{\mathrm{r} \cdot\left\{2 \cdot(1-\mathrm{R})+\left[(1+\mathrm{r})^{\mathrm{PA}}-1\right] \cdot(1+\mathrm{R})\right\}}{2 \cdot\left[(1+\mathrm{r})^{\mathrm{PA}}-1\right]}+ \\
& (\mathrm{Lr} \cdot \mathrm{DV} \cdot \mathrm{WV} \cdot \mathrm{CVE}) \cdot \frac{\mathrm{r}}{2}+\frac{\mathrm{TFa}}{2000} \cdot\left\{\mathrm{f}_{\mathrm{OCC}} \cdot \mathrm{CCC} \cdot \mathrm{ACB}+\mathrm{f}_{\mathrm{MCA}} \cdot \mathrm{a}_{\mathrm{MCA}} \cdot\left(\frac{1,1 \cdot \mathrm{Q} \cdot \mathrm{HM} \cdot \gamma}{\eta_{\mathrm{BH}}}\right)^{\mathrm{b}_{\mathrm{MCA}}}+\right. \\
& f_{\mathrm{TAH}} \cdot\left[\frac{\mathrm{Ls}}{\mathrm{Lu}} \cdot \mathrm{a}_{\mathrm{Ts}} \cdot\left(\frac{4 \cdot \mathrm{Q}}{\pi \cdot \mathrm{Vs}}\right)^{\frac{b_{\mathrm{TS}}}{2}}+\sum_{\mathrm{j}=1}^{\mathrm{j}} \mathrm{n}_{\mathrm{j}} \cdot \mathrm{a}_{\mathrm{j}} \cdot\left(\frac{4 \cdot \mathrm{Q}}{\pi \cdot \mathrm{Vs}}\right)^{\frac{\mathrm{b}_{\mathrm{j}}}{2}}+\frac{\mathrm{Lr}}{\mathrm{Lu}} \cdot \mathbf{a}_{\mathrm{Tr}} \cdot \phi^{\mathrm{b}_{\mathrm{Tr}}}+\sum_{\ell=1}^{\mathrm{L}} \mathrm{n}_{\ell} \cdot \mathrm{a}_{\ell} \cdot \phi^{\mathrm{b}_{\ell}}\right]+ \\
& \left.\mathrm{f}_{\mathrm{BH}} \cdot \mathrm{a}_{\mathrm{BH}} \cdot(\mathrm{Q} \cdot \gamma \cdot \mathrm{HM})^{\mathrm{b}_{\mathrm{BH}}}\right\}+\mathrm{CC}_{\mathrm{u}} \cdot \mathrm{C}_{\mathrm{u}} \mathrm{C} \cdot \mathrm{TFa} \cdot\left(\frac{\mathrm{Q} \cdot \mathrm{HM} \cdot \gamma}{\eta_{\mathrm{BH}}}\right)
\end{aligned}
$$

lembrando sempre que:

$$
\mathrm{HM}=\mathrm{HR}+\mathrm{PF}+5,912 \cdot \frac{\mathrm{Vs}^{2,436} \cdot \mathrm{Ls}}{\mathrm{Q}^{0,584} \cdot \mathrm{Cs}^{1,852}}+\sum_{\mathrm{j}=1}^{\mathrm{J}} \mathrm{n}_{\mathrm{j}} \cdot \mathrm{K}_{\mathrm{j}} \cdot \frac{\mathrm{Vs}^{2}}{2 \cdot \mathrm{g}}+10,649 \cdot \frac{\mathrm{Q}^{1,852} \cdot \mathrm{Lr}}{\mathrm{Cr}^{1,852} \cdot \phi^{4,871}}+\sum_{\ell=1}^{\mathrm{L}} \mathrm{n}_{\ell} \cdot \mathrm{K}_{\ell} \cdot \frac{8 \cdot \mathrm{Q}^{2}}{\mathrm{~g} \cdot \pi \cdot \phi^{4}}
$$




\subsubsection{Sistema com bomba hidráulica acionada por motor elétrico na tarifa conven-} cional sem o beneficio da Portaria 105 do DNAEE (ECN)

Fazendo-se todas substituições a equação (3.1) torna-se:

$$
\begin{gathered}
\text { CATS }=\text { CFAS }+ \text { CAMR }+(12-\mathrm{d}) \cdot\left(\frac{\mathrm{Q} \cdot \mathrm{HM} \cdot \gamma}{1000 \cdot \eta_{\mathrm{ME}} \cdot \eta_{\mathrm{BH}}}\right) \cdot \mathrm{TDc}+ \\
0,10 \cdot \mathrm{d} \cdot\left(\frac{\mathrm{Q} \cdot \mathrm{HM} \cdot \gamma}{1000 \cdot \eta_{\mathrm{ME}} \cdot \eta_{\mathrm{BH}}}\right) \cdot \mathrm{TDc}+\mathrm{TCc} \cdot\left(\frac{\mathrm{Q} \cdot \mathrm{HM} \cdot \gamma}{1000 \cdot \eta_{\mathrm{ME}} \cdot \eta_{\mathrm{BH}}}\right) \cdot \mathrm{TFd} \cdot \mathrm{NDa}
\end{gathered}
$$

sendo que:

$$
\begin{aligned}
& \text { CFAS }=\left\{\frac{L s}{L u} \cdot a_{T s} \cdot\left(\frac{4 \cdot Q}{\pi \cdot V S}\right)^{\frac{b_{n s}}{2}}+\sum_{j=1}^{J} n_{j} \cdot a_{j} \cdot\left(\frac{4 \cdot Q}{\pi \cdot V S}\right)^{\frac{b_{j}}{2}}+\frac{L r}{L u} \cdot a_{T r} \cdot \phi^{b_{\mathrm{Tr}}}+\sum_{\ell=1}^{L} n_{\ell} \cdot a_{\ell} \cdot \phi^{b_{\ell}}+a_{B H} \cdot(Q \cdot \gamma \cdot H M)^{b_{\mathrm{BH}}}+\right. \\
& \text { LLAT.CMLAT }+a_{M E} \cdot\left(\frac{1,1 \cdot Q \cdot \gamma \cdot H M}{\eta_{\mathrm{BH}}}\right)^{b_{\mathrm{ME}}}+a_{\mathrm{TE}} \cdot\left(\frac{1,1 \cdot \mathrm{Q} \cdot \gamma \cdot \mathrm{HM}}{\eta_{\mathrm{ME}} \cdot \cos \varphi \cdot \eta_{\mathrm{BH}}}\right)^{\mathrm{b}_{\mathrm{TE}}}+\mathrm{a}_{\mathrm{CP}} \cdot\left(\frac{1,1 \cdot \mathrm{Q} \cdot \gamma \cdot \mathrm{HM}}{\eta_{\mathrm{BH}}}\right)^{\mathrm{b}_{\mathrm{CP}}}+ \\
& a_{\mathrm{BC}} \cdot\left(\frac{1,1 \cdot[\operatorname{tg}(\operatorname{arc} \cos \varphi)-0,402716] \cdot \mathrm{Q} \cdot \gamma \cdot \mathrm{HM}}{\eta_{\mathrm{BH}}}\right)^{\mathrm{b}_{\mathrm{BC}}}+\mathrm{f}_{\mathrm{TE}} \cdot \mathrm{a}_{\mathrm{TE}} \cdot\left(\frac{1,1 \cdot \mathrm{Q} \cdot \gamma \cdot \mathrm{HM}}{\eta_{\mathrm{ME}} \cdot \cos \varphi \cdot \eta_{\mathrm{BH}}}\right)^{b_{\mathrm{TE}}}+ \\
& \text { CCC.ACB }\} \cdot \frac{r \cdot\left\{2 \cdot(1-R)+\left[(1+r)^{\mathrm{PA}}-1\right] \cdot(1+\mathrm{R})\right\}}{2 \cdot\left[(1+r)^{\mathrm{PA}}-1\right]}+(\mathrm{Lr} \text {. DV . WV . CVE }) \cdot \frac{\mathrm{r}}{2}
\end{aligned}
$$

e:

$$
\begin{aligned}
& \mathrm{CAMR}=\frac{\mathrm{TFa}}{2000} \cdot\left\{f_{\mathrm{THA}} \cdot\left[\frac{\mathrm{Ls}}{\mathrm{Lu}} \cdot \mathrm{a}_{\mathrm{Ts}} \cdot\left(\frac{4 \cdot \mathrm{Q}}{\pi \cdot \mathrm{Vs}}\right)^{\frac{\mathrm{b}_{\mathrm{n}}}{2}}+\sum_{\mathrm{j}=1}^{\mathrm{J}} \mathrm{n}_{\mathrm{j}} \cdot \mathrm{a}_{\mathrm{j}} \cdot\left(\frac{4 \cdot \mathrm{Q}}{\pi \cdot \mathrm{Vs}}\right)^{\frac{\mathrm{b}_{\mathrm{j}}}{2}}+\frac{\mathrm{Lr}}{\mathrm{Lu}} \cdot \mathrm{a}_{\mathrm{Tr}} \cdot \phi^{\mathrm{b}_{\mathrm{Tr}}}+\sum_{\ell=1}^{\mathrm{L}} \mathrm{n}_{\ell} \cdot \mathrm{a}_{\ell} \cdot \phi^{\mathrm{b}_{\ell}}\right]+\right. \\
& \mathrm{f}_{\mathrm{BH}} \cdot \mathrm{a}_{\mathrm{BH}} \cdot(\mathrm{Q} \cdot \gamma \cdot \mathrm{HM})^{\mathrm{b}_{\mathrm{BH}}}+\mathrm{f}_{\mathrm{ME}} \cdot \mathrm{a}_{\mathrm{ME}} \cdot\left(\frac{1,1 \cdot \mathrm{Q} \cdot \gamma \cdot \mathrm{HM}}{\eta_{\mathrm{BH}}}\right)^{\mathrm{b}_{\mathrm{XE}}}+\mathrm{f}_{\mathrm{LAT}} \cdot \text { LLAT.CMLAT }+
\end{aligned}
$$

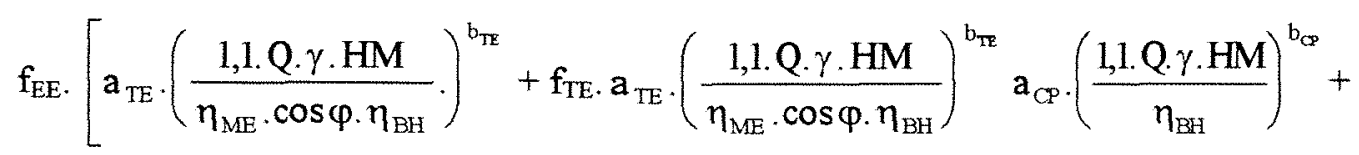

$$
\begin{aligned}
& \left.\left.a_{B C} \cdot\left(\frac{1,1 \cdot[\operatorname{tg}(\operatorname{arc} \cos \varphi)-0,402716] \cdot Q \cdot \gamma \cdot H M}{\eta_{B H}}\right)^{b_{B C}}\right]+f_{O C C} \cdot C C C \cdot A C B\right\}
\end{aligned}
$$

Caso o sistema não possua banco de capacitores para a correção do fator de potência, deve-se excluir da equação (3.59) o termo $i$ e acrescentar o termo $i i$, ou seja, o ajuste (AJ) respectivo, que corresponde a equação (3.38): 
i)

$$
\begin{aligned}
& a_{B C} \cdot\left(\frac{1,1 \cdot[\operatorname{tg}(\operatorname{arc} \cos \varphi)-0,402716] \cdot Q \cdot \gamma \cdot H M}{\eta_{B H}}\right)^{b_{B C}} \\
& \text { ii) } \quad\left[(12-\mathrm{d}) \cdot\left(\frac{\mathrm{Q} \cdot \mathrm{HM} \cdot \gamma}{1000 \cdot \eta_{\mathrm{ME}} \cdot \eta_{\mathrm{BH}}}\right) \cdot \mathrm{TDc}+\mathrm{TCc} \cdot\left(\frac{\mathrm{Q} \cdot \mathrm{HM} \cdot \gamma}{1000 \cdot \eta_{\mathrm{ME}} \cdot \eta_{\mathrm{BH}}}\right) \cdot \mathrm{TFd} \cdot \mathrm{NDa}\right] \cdot\left(\frac{0,92}{\cos \varphi}-1\right)
\end{aligned}
$$

3.3.3. Sistema com bomba hidráulica acionada por motor elétrico na tarifa convencional com o beneficio da Portaria 105 do DNAEE (ECI)

Fazendo-se todas substituições a equação (3.1) torna-se:

$$
\begin{gathered}
\text { CATS }=\text { CFAS }+ \text { CAMR }+ \\
(12-\mathrm{d}) \cdot\left(\frac{\mathrm{Q} \cdot \mathrm{HM} \cdot \gamma}{1000 \cdot \eta_{\mathrm{ME}} \cdot \eta_{\mathrm{BE}}}\right) \cdot \mathrm{TDc}+0,10 \cdot \mathrm{d} \cdot\left(\frac{\mathrm{Q} \cdot \mathrm{HM} \cdot \gamma}{1000 \cdot \eta_{\mathrm{ME}} \cdot \eta_{\mathrm{BH}}}\right) \cdot \mathrm{TDc}+ \\
\text { TCc. }\left(\frac{\mathrm{Q} \cdot \mathrm{HM} \cdot \gamma}{1000 \cdot \eta_{\mathrm{ME}} \cdot \eta_{\mathrm{BH}}}\right) \cdot(\mathrm{TFd}-\mathrm{TFdhe} \cdot \mathrm{fdtc}) \cdot \mathrm{NDa}
\end{gathered}
$$

Caso o sistema não possua banco de capacitores para a correção do fator de potência, deve-se excluir da equação (3.60) o termo $i$ e acrescentar o termo $i i$, ou seja, o ajuste (AJ) respectivo, que corresponde a equação (3.39):

i)

$$
a_{B C} \cdot\left(\frac{1,1 \cdot[\operatorname{tg}(\operatorname{arc} \cos \varphi)-0,402716] \cdot Q \cdot \gamma \cdot H M}{\eta_{\mathrm{BH}}}\right)^{b_{B C}}
$$

ii) $\left[(12-\mathrm{d}) \cdot\left(\frac{\mathrm{Q} \cdot \mathrm{HM} \cdot \gamma}{1000 \cdot \eta_{\mathrm{ME}} \cdot \eta_{\mathrm{BH}}}\right) \cdot \mathrm{TDc}+\mathrm{TCc} \cdot\left(\frac{\mathrm{Q} \cdot \mathrm{HM} \cdot \gamma}{1000 \cdot \eta_{\mathrm{ME}} \cdot \eta_{\mathrm{BH}}}\right) \cdot(\mathrm{TFd}-\mathrm{TFdhe} \cdot \mathrm{fdtc}) \cdot \mathrm{NDa}\right] \cdot\left(\frac{0,92}{\cos \varphi}-1\right)$

\subsubsection{Sistema com bomba hidráulica acionada por motor elétrico na tarifa verde sem o benefício da Portaria 105 do DNAEE (EVN)}

Fazendo-se todas substituições a equação (3.1) torna-se:

$$
\begin{gathered}
\text { CATS }=\text { CFAS }+ \text { CAMR }+ \\
(12-d) \cdot\left(\frac{\text { Q.HM. } \gamma}{1000 \cdot \eta_{\mathrm{ME}} \cdot \eta_{\mathrm{BH}}}\right) \cdot \operatorname{TDv}+0,10 \cdot \mathrm{d} \cdot\left(\frac{\mathrm{Q} \cdot \mathrm{HM} \cdot \gamma}{1000 \cdot \eta_{\mathrm{ME}} \cdot \eta_{\mathrm{BH}}}\right) \cdot \mathrm{TDv}+ \\
\text { TCvup. }\left(\frac{\mathrm{Q} \cdot \mathrm{HM} \cdot \gamma}{1000 \cdot \eta_{\mathrm{ME}} \cdot \eta_{\mathrm{BH}}}\right) \cdot \mathrm{TFdp} \cdot \mathrm{NDu} \cdot \frac{5}{7}+\mathrm{TCvufp} \cdot\left(\frac{\mathrm{Q} \cdot \mathrm{HM} \cdot \gamma}{1000 \cdot \eta_{\mathrm{ME}} \cdot \eta_{\mathrm{BH}}}\right) \cdot\left(\mathrm{TFdfp}+\mathrm{TFdp} \cdot \frac{2}{7}\right) \cdot \mathrm{NDu}+
\end{gathered}
$$


$\operatorname{TCvsp} \cdot\left(\frac{\mathrm{Q} \cdot \mathrm{HM} \cdot \gamma}{1000 \cdot \eta_{\mathrm{ME}} \cdot \eta_{\mathrm{BH}}}\right) \cdot$ TFdp. NDs. $\frac{5}{7}+$ TCvsfp $\cdot\left(\frac{\text { Q.HM. } \gamma}{1000 \cdot \eta_{\mathrm{XE}} \cdot \eta_{\mathrm{BH}}}\right) \cdot\left(\right.$ TFdfp + TFdp $\left.\frac{2}{7}\right) \cdot$ NDs (3.61)

Caso o sistema não possua banco de capacitores para a correção do fator de potência, deve-se excluir da equação (3.61) o termo $i$ e acrescentar o termo $i i$, ou seja, o ajuste (AJ) respectivo, que corresponde a equação (3.50):

i)

$$
a_{B C} \cdot\left(\frac{1,1 \cdot[\operatorname{tg}(\operatorname{arc} \cos \varphi)-0,402716] \cdot Q \cdot \gamma \cdot H M}{\eta_{B H}}\right)^{b_{B C}}
$$

ii) $\left[\right.$ TCvup. $\left(\frac{\text { Q.HM } \cdot \gamma}{1000 \cdot \eta_{\mathrm{ME}} \cdot \eta_{\mathrm{BH}}}\right) \cdot$ TFdp.NDu. $\frac{5}{7}+$ TCvufp. $\left(\frac{\text { Q.HM } \cdot \gamma}{1000 \cdot \eta_{\mathrm{ME}} \cdot \eta_{\mathrm{BH}}}\right) \cdot\left(\right.$ TFdfp + TFdp. $\left.\frac{2}{7}\right) \cdot \mathrm{NDu}+$ TCvsp. $\left(\frac{\text { Q.HM. } \gamma}{1000 \cdot \eta_{\mathrm{ME}} \cdot \eta_{\mathrm{BH}}}\right) \cdot$ TFdp.NDs. $\frac{5}{7}+$ TCvsfp. $\left(\frac{\text { Q.HM. } \gamma}{1000 \cdot \eta_{\mathrm{ME}} \cdot \eta_{\mathrm{BH}}}\right) \cdot\left(\operatorname{TFdfp}+\right.$ TFdp $\left.\frac{2}{7}\right) \cdot \mathrm{NDs}+$ $\left.(12-\mathrm{d}) \cdot\left(\frac{\mathrm{Q} \cdot \mathrm{HM} \cdot \gamma}{1000 \cdot \eta_{\mathrm{ME}} \cdot \eta_{\mathrm{BH}}}\right) \cdot \mathrm{TDV}\right] \cdot\left(\frac{0,92}{\cos \varphi}-1\right)$

\subsubsection{Sistema com bomba hidráulica acionada por motor elétrico na tarifa verde com o benefício da Portaria 105 do DNAEE (EVI)}

Fazendo-se todas substituições a equação (3.1) torna-se:

$$
\begin{aligned}
& \text { CATS }=\text { CFAS + CAMR + } \\
& (12-\mathrm{d}) \cdot\left(\frac{\mathrm{Q} \cdot \mathrm{HM} \cdot \gamma}{1000 \cdot \eta_{\mathrm{ME}} \cdot \eta_{\mathrm{BH}}}\right) \cdot \mathrm{TDv}+0,10 \cdot \mathrm{d} \cdot\left(\frac{\mathrm{Q} \cdot \mathrm{HM} \cdot \gamma}{1000 \cdot \eta_{\mathrm{ME}} \cdot \eta_{\mathrm{BH}}}\right) \cdot \mathrm{TDv}+ \\
& \text { TCvup. }\left(\frac{\text { Q.HM. } \gamma}{1000 \cdot \eta_{\mathrm{ME}} \cdot \eta_{\mathrm{BH}}}\right) \cdot \text { TFdp.NDu. } \frac{5}{7}+ \\
& \text { TCvufp. }\left(\frac{\text { Q.HM. } \gamma}{1000 \cdot \eta_{\mathrm{ME}} \cdot \eta_{\mathrm{BH}}}\right) \cdot\left[\left(\text { TFdfp }+ \text { TFdp } \frac{2}{7}\right)-\text { TFdhe. fdtc }\right] \text { NDu }+ \\
& \text { TCvsp. }\left(\frac{\text { Q.HM. } \gamma}{1000 \cdot \eta_{\mathrm{ME}} \cdot \eta_{\mathrm{BH}}}\right) \cdot \text { TFdp. NDs. } \frac{5}{7}+ \\
& \text { TCvsfp. }\left(\frac{\text { Q.HM. } \gamma}{1000 \cdot \eta_{\mathrm{ME}} \cdot \eta_{\mathrm{BH}}}\right) \cdot\left[\left(\text { TFdfp }+ \text { TFdp. } \frac{2}{7}\right)-\text { TFdhe.fdtc }\right] \text { NDs }
\end{aligned}
$$

Caso o sistema não possua banco de capacitores para a correção do fator de potência, deve-se excluir da equação (3.62) o termo $i$ e acrescentar o termo $i i$, ou seja, o ajuste (AJ) respectivo, que corresponde a equação (3.51): 
i)

$$
a_{\mathrm{BC}} \cdot\left(\frac{1,1 \cdot[\operatorname{tg}(\operatorname{arc} \cos \varphi)-0,402716] \cdot \mathrm{Q} \cdot \gamma \cdot \mathrm{HM}}{\eta_{\mathrm{BH}}}\right)^{b_{\mathrm{BC}}}
$$

ii)

$$
\begin{aligned}
& {\left[\text { TCvup. }\left(\frac{\text { Q.HM. } \gamma}{1000 \cdot \eta_{\mathrm{ME}} \cdot \eta_{\mathrm{BH}}}\right) \cdot \text { TFdp. NDu. } \frac{5}{7}\right)+} \\
& \text { TCvufp. }\left(\frac{\text { Q.HM. } \gamma}{1000 \cdot \eta_{\mathrm{ME}} \cdot \eta_{\mathrm{BH}}}\right) \cdot\left[\left(\text { TFdfp }+ \text { TFdp. } \frac{2}{7}\right)-\text { TFdhe. fdtc }\right] \mathrm{NDu}+ \\
& \operatorname{TCvsp} \cdot\left(\frac{\text { Q.HM. } \gamma}{1000 \cdot \eta_{\mathrm{ME}} \cdot \eta_{\mathrm{BH}}}\right) \cdot \text { TFdp. NDs. } \frac{5}{7}+ \\
& \text { TCvsfp. }\left(\frac{\text { Q.HM. } \gamma}{1000 \cdot \eta_{\mathrm{ME}} \cdot \eta_{\mathrm{BE}}}\right) \cdot\left[\left(\text { TFdfp }+ \text { TFdp } \frac{2}{7}\right)-\text { TFdhe.fdtc }\right] \text { NDs }+ \\
& \left.(12-\mathrm{d}) \cdot\left(\frac{\mathrm{Q} \cdot \mathrm{HM} \cdot \gamma}{1000 \cdot \eta_{\mathrm{ME}} \cdot \eta_{\mathrm{BH}}}\right) \cdot \operatorname{TDv}\right] \cdot\left(\frac{0,92}{\cos \varphi}-1\right)
\end{aligned}
$$

\subsubsection{Sistema com bomba hidráulica acionada por motor elétrico na tarifa azul sem} o benefício da Portaria 105 do DNAEE (EAN)

Fazendo-se todas substituições a equação (3.1) torna-se:

$$
\begin{aligned}
& \text { CATS }=\text { CFAS }+ \text { CAMR }+ \\
& (5-\operatorname{dup}) \cdot\left(\frac{\mathrm{Q} \cdot \mathrm{HM} \cdot \gamma}{1000 \cdot \eta_{\mathrm{ME}} \cdot \eta_{\mathrm{BH}}}\right) \cdot \text { TDap }+0,10 \cdot d u p \cdot\left(\frac{\mathrm{Q} \cdot \mathrm{HM} \cdot \gamma}{1000 \cdot \eta_{\mathrm{ME}} \cdot \eta_{\mathrm{BH}}}\right) \cdot \text { TDap }+ \\
& (5-\text { dufp }) \cdot\left(\frac{\text { Q.HM } \cdot \gamma}{1000 \cdot \eta_{\mathrm{ME}} \cdot \eta_{\mathrm{BH}}}\right) \cdot \operatorname{TDafp}+0,10 \cdot \mathrm{dufp} \cdot\left(\frac{\mathrm{Q} \cdot \mathrm{HM} \cdot \gamma}{1000 \cdot \eta_{\mathrm{ME}} \cdot \eta_{\mathrm{BH}}}\right) \cdot \text { TDafp }+ \\
& (7-\mathrm{dsp}) \cdot\left(\frac{\mathrm{Q} \cdot \mathrm{HM} \cdot \gamma}{1000 \cdot \eta_{\mathrm{ME}} \cdot \eta_{\mathrm{BH}}}\right) \cdot \operatorname{TDap}+0,10 \cdot \mathrm{dsp} \cdot\left(\frac{\mathrm{Q} \cdot \mathrm{HM} \cdot \gamma}{1000 \cdot \eta_{\mathrm{ME}} \cdot \eta_{\mathrm{BH}}}\right) \cdot \mathrm{TDap}+ \\
& (7-\text { dsfp }) \cdot\left(\frac{\text { Q.HM } \cdot \gamma}{1000 \cdot \eta_{\mathrm{ME}} \cdot \eta_{\mathrm{BH}}}\right) \cdot \text { TDafp }+0,10 \cdot \mathrm{dsfp} \cdot\left(\frac{\mathrm{Q} \cdot \mathrm{HM} \cdot \gamma}{1000 \cdot \eta_{\mathrm{ME}} \cdot \eta_{\mathrm{BH}}}\right) \cdot \text { TDafp }+
\end{aligned}
$$

TCaup. $\left(\frac{\text { Q.HM. } \gamma}{1000 \cdot \eta_{\mathrm{ME}} \cdot \eta_{\mathrm{BH}}}\right) \cdot$ TFdp. NDu. $\frac{5}{7}+$ TCaufp. $\left(\frac{\text { Q.HM. } \gamma}{1000 \cdot \eta_{\mathrm{ME}} \cdot \eta_{\mathrm{BH}}}\right) \cdot\left(\right.$ TFdfp + TFdp. $\left.\frac{2}{7}\right) \cdot \mathrm{NDu}+$ TCasp. $\left(\frac{\text { Q.HM. } \gamma}{1000 \cdot \eta_{\mathrm{ME}} \cdot \eta_{\mathrm{BH}}}\right) \cdot$ TFdp.NDs. $\frac{5}{7}+$ TCasfp. $\left(\frac{\text { Q.HM. } \gamma}{1000 \cdot \eta_{\mathrm{ME}} \cdot \eta_{\mathrm{BH}}}\right) \cdot\left(\right.$ TFdfp + TFdp $\left.\frac{2}{7}\right) \cdot$ NDs (3.63)

Caso o sistema não possua banco de capacitores para a correção do fator de potência, deve-se excluir da equação (3.63) o termo $i$ e acrescentar o termo $i i$, ou seja, o ajuste (AJ) respectivo, que corresponde a equação (3.56): 
i)

$$
a_{\mathrm{BC}} \cdot\left(\frac{1,1 \cdot[\operatorname{tg}(\operatorname{arc} \cos \varphi)-0,402716] \cdot \mathrm{Q} \cdot \gamma \cdot \mathrm{HM}}{\eta_{\mathrm{BH}}}\right)^{b_{\mathrm{BC}}}
$$

ii) $\quad\left[(5-\right.$ dup $) \cdot\left(\frac{\text { Q.HM } \cdot \gamma}{1000 \cdot \eta_{\mathrm{ME}} \cdot \eta_{\mathrm{BH}}}\right) \cdot$ TDap $+(5-$ dufp $) \cdot\left(\frac{\text { Q.HM } \cdot \gamma}{1000 \cdot \eta_{\mathrm{ME}} \cdot \eta_{\mathrm{BH}}}\right) \cdot$ TDafp + $(7-\mathrm{dsp}) \cdot\left(\frac{\mathrm{Q} \cdot \mathrm{HM} \cdot \gamma}{1000 \cdot \eta_{\mathrm{ME}} \cdot \eta_{\mathrm{BH}}}\right) \cdot$ TDap $+(7-\mathrm{dsfp}) \cdot\left(\frac{\mathrm{Q} \cdot \mathrm{HM} \cdot \gamma}{1000 \cdot \eta_{\mathrm{ME}} \cdot \eta_{\mathrm{BH}}}\right) \cdot$ TDafp +

TCaup. $\left(\frac{\text { Q.HM. } \gamma}{1000 \cdot \eta_{\mathrm{ME}} \cdot \eta_{\mathrm{BH}}}\right) \cdot$ TFdp.NDu $\frac{5}{7}+$ TCaufp. $\left(\frac{\text { Q.HM. } \gamma}{1000 \cdot \eta_{\mathrm{ME}} \cdot \eta_{\mathrm{BH}}}\right) \cdot\left(\operatorname{TFdfp}+\operatorname{TFdp} \cdot \frac{2}{7}\right) \cdot \mathrm{NDu}+$ $\left.\operatorname{TCasp} \cdot\left(\frac{\mathrm{Q} \cdot \mathrm{HM} \cdot \gamma}{1000 \cdot \eta_{\mathrm{ME}} \cdot \eta_{\mathrm{BH}}}\right) \cdot \operatorname{TFdp} \cdot \mathrm{NDs} \cdot \frac{5}{7}+\operatorname{TCasfp} \cdot\left(\frac{\mathrm{Q} \cdot \mathrm{HM} \gamma}{1000 \cdot \eta_{\mathrm{ME}} \cdot \eta_{\mathrm{BH}}}\right) \cdot\left(\operatorname{TFdfp}+\operatorname{TFdp} \cdot \frac{2}{7}\right) \cdot \operatorname{NDs}\right]\left(\frac{0,92}{\cos \varphi}-1\right)$

\subsubsection{Sistema com bomba hidráulica acionada por motor elétrico na tarifa azul com o beneficio da Portaria 105 do DNAEE (EAI)}

$$
\begin{aligned}
& \text { Fazendo-se todas substituições a equação (3.1) torna-se: } \\
& \text { CATS }=\text { CFAS }+ \text { CAMR }+ \\
& (5-\operatorname{dup}) \cdot\left(\frac{\mathrm{Q} \cdot \mathrm{HM} \cdot \gamma}{1000 \cdot \eta_{\mathrm{ME}} \cdot \eta_{\mathrm{BH}}}\right) \cdot \operatorname{TDap}+0,10 \cdot \mathrm{dup} \cdot\left(\frac{\mathrm{Q} \cdot \mathrm{HM} \cdot \gamma}{1000 \cdot \eta_{\mathrm{ME}} \cdot \eta_{\mathrm{BH}}}\right) \cdot \text { TDap }+ \\
& \left(5-\text { dufp) } \cdot\left(\frac{\text { Q.HM } \cdot \gamma}{1000 \cdot \eta_{\mathrm{ME}} \cdot \eta_{\mathrm{BH}}}\right) \cdot \operatorname{TDafp}+0,10 \cdot \mathrm{dufp} \cdot\left(\frac{\mathrm{Q} \cdot \mathrm{HM} \cdot \gamma}{1000 \cdot \eta_{\mathrm{ME}} \cdot \eta_{\mathrm{BH}}}\right) \cdot \text { TDafp }+\right. \\
& (7-\mathrm{dsp}) \cdot\left(\frac{\mathrm{Q} \cdot \mathrm{HM} \cdot \gamma}{1000 \cdot \eta_{\mathrm{ME}} \cdot \eta_{\mathrm{BH}}}\right) \cdot \operatorname{TDap}+0,10 \cdot \mathrm{dsp} \cdot\left(\frac{\mathrm{Q} \cdot \mathrm{HM} \cdot \gamma}{1000 \cdot \eta_{\mathrm{ME}} \cdot \eta_{\mathrm{BH}}}\right) \cdot \mathrm{TDap}+ \\
& (7-\mathrm{dsfp}) \cdot\left(\frac{\mathrm{Q} \cdot \mathrm{HM} \cdot \gamma}{1000 \cdot \eta_{\mathrm{ME}} \cdot \eta_{\mathrm{BH}}}\right) \cdot \text { TDafp }+0,10 \cdot \mathrm{dsfp} \cdot\left(\frac{\mathrm{Q} \cdot \mathrm{HM} \cdot \gamma}{1000 \cdot \eta_{\mathrm{ME}} \cdot \eta_{\mathrm{BH}}}\right) \cdot \text { TDafp }+ \\
& \text { TCaup. }\left(\frac{\text { Q.HM. } \gamma}{1000 \cdot \eta_{\mathrm{ME}} \cdot \eta_{\mathrm{BH}}}\right) \cdot \text { TFdp.NDu. } \frac{5}{7}+ \\
& \text { TCaufp. }\left(\frac{\text { Q.HM. } \gamma}{1000 \cdot \eta_{\mathrm{ME}} \cdot \eta_{\mathrm{BH}}}\right) \cdot\left[\left(\text { TFdfp }+ \text { TFdp. } \frac{2}{7}\right)-\text { TFdhe. fdtc }\right] \text { NDu }+ \\
& \text { TCasp. }\left(\frac{\text { Q.HM. } \gamma}{1000 \cdot \eta_{\mathrm{ME}} \cdot \eta_{\mathrm{BH}}}\right) \text { TFdp. NDs. } \frac{5}{7}+ \\
& \text { TCasfp. }\left(\frac{\text { Q.HM. } \gamma}{1000 \cdot \eta_{\mathrm{ME}} \cdot \eta_{\mathrm{BH}}}\right) \cdot\left[\left(\text { TFdfp }+ \text { TFdp. } \frac{2}{7}\right)-\text { TFdhe. fdtc }\right] \text { NDs }
\end{aligned}
$$


Caso o sistema não possua banco de capacitores para a correção do fator de potência, deve-se excluir da equação (3.64) o termo $i$ e acrescentar o termo $i i$, ou seja, o ajuste (AJ) respectivo, que corresponde a equação (3.57):

i)

$$
\begin{aligned}
& \mathrm{a}_{\mathrm{BC}} \cdot\left(\frac{1,1 \cdot[\operatorname{tg}(\operatorname{arc} \cos \varphi)-0,402716] \cdot \mathrm{Q} \cdot \gamma \cdot \mathrm{HM}}{\eta_{\mathrm{BH}}}\right)^{\mathrm{b}_{\mathrm{BC}}} \\
& \text { ii) } \quad\left[(5-\text { dup }) \cdot\left(\frac{\text { Q.HM. } \gamma}{1000 \cdot \eta_{\mathrm{ME}} \cdot \eta_{\mathrm{BH}}}\right) \cdot \operatorname{TDap}+(5-\mathrm{dufp}) \cdot\left(\frac{\mathrm{Q} \cdot \mathrm{HM} \cdot \gamma}{1000 \cdot \eta_{\mathrm{ME}} \cdot \eta_{\mathrm{BH}}}\right) \cdot \text { TDafp }+\right. \\
& (7-\mathrm{dsp}) \cdot\left(\frac{\mathrm{Q} \cdot \mathrm{HM} \cdot \gamma}{1000 \cdot \eta_{\mathrm{ME}} \cdot \eta_{\mathrm{BH}}}\right) \cdot \text { TDap }+(7-\mathrm{dsfp}) \cdot\left(\frac{\mathrm{Q} \cdot \mathrm{HM} \cdot \gamma}{1000 \cdot \eta_{\mathrm{ME}} \cdot \eta_{\mathrm{BH}}}\right) \cdot \text { TDafp }+ \\
& \text { TCaup. }\left(\frac{\text { Q.HM. } \gamma}{1000 \cdot \eta_{\mathrm{ME}} \cdot \eta_{\mathrm{BH}}}\right) \cdot \text { TFdp.NDu. } \frac{5}{7}+ \\
& \text { TCaufp. }\left(\frac{\mathrm{Q} \cdot \mathrm{HM} \cdot \gamma}{1000 \cdot \eta_{\mathrm{ME}} \cdot \eta_{\mathrm{BH}}}\right) \cdot\left[\left(\text { TFdfp }+ \text { TFdp } \cdot \frac{2}{7}\right)-\text { TFdhe. fdtc }\right] \text { NDu }+ \\
& \text { TCasp. }\left(\frac{Q \cdot H M \cdot \gamma}{1000 \cdot \eta_{\mathrm{ME}} \cdot \eta_{\mathrm{BH}}}\right) \cdot \text { TFdp.NDs. } \frac{5}{7}+ \\
& \text { TCasfp. } \left.\left(\frac{Q \cdot H M \cdot \gamma}{1000 \cdot \eta_{\mathrm{ME}} \cdot \eta_{\mathrm{BH}}}\right) \cdot\left[\left(\operatorname{TFdfp}+\text { TFdp } \frac{2}{7}\right)-\text { TFdhe.fdtc }\right] \text { NDs }\right]\left(\frac{0,92}{\cos \varphi}-1\right)
\end{aligned}
$$

\subsection{Modelo para cálculo do diâmetro econômico da tubulação de recalque}

As equações (3.58), (3.59), (3.60), (3.61), (3.62), (3.63) e (3.64) estimam o custo anual total do sistema de recalque (CATS) em qualquer diâmetro contemplado para a tubulação de recalque, sendo porém o mais econômico aquele obtido quando $\mathrm{dCATS} / \mathrm{d} \phi=0$. Todavia, é fácil perceber a dificuldade de se fazer tal operação nestas equações, o que torna o processo bastante trabalhoso. Com isso, a solução poderia ser obtida por tentativas, o que também incorreria em longa tarefa. Diante de tais colocações, neste item é proposto uma maneira alternativa de encontrar seu valor aproximado $\left(\phi_{\mathrm{e}}\right)$.

Assumindo-se que as perdas de carga localizadas na tubulação de recalque são despreziveis, uma vez que $\mathrm{Lr}>4000 \phi$ na maioria das vezes, pode-se representar o custo anual total do sistema, sem estes efeitos, por CATS*. Sendo assim, o diâmetro econômico da tubulação de recalque pode ser obtido fazendo-se dCATS $* / d \phi=0$ nas equações $(3.58),(3.59),(3.60),(3.61)$, 
(3.62), (3.63) e (3.64), ou seja, excluindo-se nestas o termo referente às perdas de carga localizadas, que está implícito na altura manométrica total (HM):

$$
\sum_{\ell=1}^{L} n_{\imath} \cdot K_{\ell} \cdot \frac{8 \cdot Q^{2}}{\mathrm{~g} \cdot \pi \cdot \phi^{4}} \quad \text { (perdas de carga localizadas no recalque) }
$$

\subsubsection{Sistema com bomba hidráulica acionada por motor à combustão (D)}

Com base na equação (3.58) tem-se:

$$
\begin{gathered}
\frac{\mathrm{dCATS} *}{\mathrm{~d} \phi}=0 \Rightarrow\left[\left(\frac{\mathrm{Lr}}{\mathrm{Lu}} \cdot \mathrm{a}_{\mathrm{TH}} \cdot \mathrm{b}_{\mathrm{TH}} \cdot \phi^{\mathrm{b}_{\mathrm{TH}}^{-1}}+\sum_{\ell=1}^{\mathrm{L}} \mathrm{n}_{\ell} \cdot \mathrm{a}_{\ell} \cdot \mathrm{b}_{\ell} \cdot \phi^{\mathrm{b}_{\ell}-1}\right)+\right. \\
\left.\frac{1,1 \cdot \mathrm{a}_{\mathrm{MCA}} \cdot \mathrm{b}_{\mathrm{MCA}}}{\eta_{\mathrm{BH}}} \cdot \beta \cdot\left(\frac{1,1}{\eta_{\mathrm{BH}}} \cdot \lambda\right)^{\mathrm{b}_{\mathrm{MCA}}-1}+\beta \cdot \mathrm{a}_{\mathrm{BH}} \cdot \mathrm{b}_{\mathrm{BH}} \cdot \lambda^{\mathrm{b}_{\mathrm{BH}}-1}\right] \cdot\left\{\frac{r \cdot\left\{2 \cdot(1-\mathrm{R})+\left[(1+\mathrm{r})^{\mathrm{PA}}-1\right] \cdot(1+\mathrm{R})\right\}}{2 \cdot\left[(1+\mathrm{r})^{\mathrm{PA}}-1\right]+}\right. \\
\frac{\mathrm{TFa}}{2000} \cdot\left[\frac{1,1 \cdot \mathrm{f}_{\mathrm{MCA}} \cdot \mathrm{a}_{\mathrm{MCA}} \cdot \mathrm{b}_{\mathrm{MCA}}}{\eta_{\mathrm{BH}}} \cdot \beta \cdot\left(\frac{1,1}{\eta_{\mathrm{BH}}} \cdot \lambda\right)^{\mathrm{b}_{\mathrm{MCA}}-1}+\beta \cdot \mathrm{f}_{\mathrm{BH}} \cdot \mathrm{a}_{\mathrm{BH}} \cdot \mathrm{b}_{\mathrm{BH}} \cdot \lambda^{\mathrm{b}_{\mathrm{BH}}-1}+\right. \\
\left.\mathrm{f}_{\mathrm{TAH}} \cdot\left(\frac{\mathrm{Lr}}{\mathrm{Lu}} \cdot \mathrm{a}_{\mathrm{TH}} \cdot \mathrm{b}_{\mathrm{TH}} \cdot \phi^{\mathrm{b}_{\mathrm{TH}}-1}+\sum_{\ell=1}^{\mathrm{L}} \mathrm{n}_{\ell} \cdot \mathrm{a}_{\ell} \cdot \mathrm{b}_{\ell} \cdot \phi^{\mathrm{b}_{\ell}-1}\right)\right]+\frac{\mathrm{CC}_{\mathrm{u}} \cdot \mathrm{C}_{\mathrm{u}} \mathrm{C} \cdot \mathrm{TFa}}{\eta_{\mathrm{BH}}} \cdot \beta=0
\end{gathered}
$$

sendo que: $\beta=-51,871 \cdot \frac{Q^{2,852} \cdot \operatorname{Lr} \cdot \gamma}{\mathrm{Cr}^{1,852} \cdot \phi^{5,871}} \quad \mathrm{e}: \lambda=10,649 \cdot \frac{\mathrm{Q}^{2,852} \cdot \operatorname{Lr} \cdot \gamma}{\mathrm{Cr}^{1,852} \cdot \phi^{4,871}}+\mathrm{Q} \cdot \gamma \cdot(\mathrm{HR}+\mathrm{PF})$

\subsubsection{Sistema com bomba hidráulica acionada por motor elétrico na tarifa conven- cional sem o beneficio da Portaria 105 do DNAEE (ECN)}

Com base na equação (3.59) tem-se:

$$
\begin{gathered}
\frac{\mathrm{dCATS}^{*}}{\mathrm{~d} \phi}=0 \Rightarrow \frac{\mathrm{dCFAS} *}{\mathrm{~d} \phi}+\frac{\mathrm{dCAMR} *}{\mathrm{~d} \phi}+(12-\mathrm{d}) \cdot \frac{1}{1000 \cdot \eta_{\mathrm{ME}} \cdot \eta_{\mathrm{BH}}} \cdot \beta \cdot \mathrm{TDc}^{*}+ \\
\quad 0,10 . \mathrm{d} \cdot \frac{1}{1000 \cdot \eta_{\mathrm{ME}} \cdot \eta_{\mathrm{BH}}} \cdot \beta \cdot \mathrm{TDc}+\mathrm{TCc} \cdot \frac{1}{1000 \cdot \eta_{\mathrm{ME}} \cdot \eta_{\mathrm{BH}}} \cdot \beta \cdot \mathrm{TFd} \cdot \mathrm{NDa}=0
\end{gathered}
$$

sendo que: $\frac{\mathrm{dCFAS}}{\mathrm{d} \phi}=\left\{\left(\frac{\mathrm{Lr}}{\mathrm{Lu}} \cdot \mathrm{a}_{\mathrm{TH}} \cdot \mathrm{b}_{\mathrm{TH}} \cdot \phi^{\mathrm{b}_{\mathrm{TH}}-1}+\sum_{\ell=1}^{\mathrm{L}} \mathrm{n}_{\ell} \cdot \mathrm{a}_{\ell} \cdot \mathrm{b}_{\ell} \cdot \phi^{\mathrm{b}_{\ell}-1}\right)+\beta \cdot \mathrm{a}_{\mathrm{BH}} \cdot \mathrm{b}_{\mathrm{BH}} \cdot \lambda^{\mathrm{b}_{\mathrm{BH}}{ }^{-1}}+\right.$

$$
\frac{1,1 \cdot a_{\mathrm{ME}} \cdot b_{\mathrm{ME}}}{\eta_{\mathrm{BH}}} \cdot \beta \cdot\left(\frac{1,1}{\eta_{\mathrm{BH}}} \cdot \lambda\right)^{\mathrm{b}_{\mathrm{ME}}-1}+\frac{1,1 \cdot a_{\mathrm{TE}} \cdot b_{\mathrm{TE}}}{\eta_{\mathrm{ME}} \cdot \cos \varphi \cdot \eta_{\mathrm{BH}}} \cdot \beta \cdot\left(\frac{1,1}{\eta_{\mathrm{BH}}} \cdot \lambda\right)^{b_{\mathrm{TE}}-1}+
$$




$$
\begin{aligned}
& \frac{1,1 \cdot a_{C P} \cdot b_{C P}}{\eta_{B H}} \cdot \beta \cdot\left(\frac{1,1}{\eta_{B H}} \cdot \lambda\right)^{b_{C \mathrm{CP}}-1}+\frac{1,1 \cdot[\operatorname{tg}(\operatorname{arc} \cos \varphi)-0,402716] \cdot a_{B C} \cdot b_{B C}}{\eta_{B H}} \cdot \beta \cdot\left(\frac{1,1}{\eta_{B H}} \cdot \lambda\right)^{b_{B C}-1}+ \\
& \left.\frac{1,1 \cdot f_{\mathrm{TE}} \cdot \mathrm{a}_{\mathrm{TE}} \cdot \mathrm{b}_{\mathrm{TE}}}{\eta_{\mathrm{ME}} \cdot \cos \varphi \cdot \eta_{\mathrm{BH}}} \cdot \beta \cdot\left(\frac{1,1}{\eta_{\mathrm{BH}}} \cdot \lambda\right)^{b_{\mathrm{TE}}-1}\right\} \cdot\left\{\frac{\mathrm{r} \cdot\left\{2 \cdot(1-\mathrm{R})+\left[(1+\mathrm{r})^{\mathrm{PA}}-1\right] \cdot(1+\mathrm{R})\right\}}{2 \cdot\left[(1+\mathrm{r})^{\mathrm{PA}}-1\right]}\right\} \\
& \text { e: } \quad \frac{\mathrm{dCAMR} *}{\mathrm{~d} \phi}=\frac{\mathrm{TFa}}{2000} \cdot\left\{\mathrm{f}_{\mathrm{TAH}} \cdot\left(\frac{\mathrm{Lr}}{\mathrm{Lu}} \cdot \mathrm{a}_{\mathrm{TH}} \cdot \mathrm{b}_{\mathrm{TH}} \cdot \phi^{\mathrm{b}_{\mathrm{TI}}^{-1}}+\sum_{\ell=1}^{\mathrm{L}} \mathrm{n}_{\ell} \cdot \mathrm{a}_{\ell} \cdot \mathrm{b}_{\ell} \cdot \phi^{\mathrm{b}_{\ell-1}}\right)+\right. \\
& f_{\mathrm{BH}} \cdot \beta \cdot \mathrm{a}_{\mathrm{BH}} \cdot b_{\mathrm{BH}} \cdot \lambda^{\mathrm{b}_{\mathrm{BH}}-1}+\frac{1,1 \cdot f_{\mathrm{ME}} \cdot \mathrm{a}_{\mathrm{ME}} \cdot b_{\mathrm{ME}}}{\eta_{\mathrm{BH}}} \cdot \beta \cdot\left(\frac{1,1}{\eta_{\mathrm{BH}}} \cdot \lambda\right)^{\mathrm{b}_{\mathrm{ME}}-1}+ \\
& f_{\mathrm{EE}} \cdot\left[\frac{1,1 \cdot a_{\mathrm{TE}} \cdot b_{\mathrm{TE}}}{\eta_{\mathrm{ME}} \cdot \cos \varphi \cdot \eta_{\mathrm{BH}}} \cdot \beta \cdot\left(\frac{1,1}{\eta_{\mathrm{BH}}} \cdot \lambda\right)^{\mathrm{b}_{\mathrm{TP}}-1}+\frac{1,1 \cdot a_{\mathrm{CP}} \cdot b_{\mathrm{CP}}}{\eta_{\mathrm{BH}}} \cdot \beta \cdot\left(\frac{1,1}{\eta_{\mathrm{BH}}} \cdot \lambda\right)^{\mathrm{b}_{\mathrm{CP}}-1}+\right. \\
& \left.\left.\frac{1,1 \cdot[\operatorname{tg}(\operatorname{arc} \cos \varphi)-0,402716] \cdot a_{\mathrm{BC}} \cdot b_{\mathrm{BC}}}{\eta_{\mathrm{BH}}} \cdot \beta \cdot\left(\frac{1,1}{\eta_{\mathrm{BH}}} \cdot \lambda\right)^{b_{\mathrm{EC}}-1}+\frac{1,1 \cdot f_{\mathrm{TE}} \cdot a_{\mathrm{TE}} \cdot b_{\mathrm{TE}}}{\eta_{\mathrm{ME}} \cdot \cos \varphi \cdot \eta_{\mathrm{BH}}} \cdot \beta \cdot\left(\frac{1,1}{\eta_{\mathrm{BH}}} \cdot \lambda\right)^{b_{\mathrm{TE}}-1}\right]\right\}
\end{aligned}
$$

Caso o sistema não possua banco de capacitores, deve-se excluir da equação (3.66) o termo implícito $i$ e acrescentar no final da mesma o termo $i i$, ou seja, a $\mathrm{dAJ} / \mathrm{d} \phi$ respectiva:

i)

$$
\frac{1,1 \cdot[\operatorname{tg}(\operatorname{arc} \cos \varphi)-0,402716] \cdot a_{\mathrm{BC}} \cdot b_{\mathrm{BC}}}{\eta_{\mathrm{BH}}} \cdot \beta \cdot\left(\frac{1,1}{\eta_{\mathrm{BH}}} \cdot \lambda\right)^{\mathrm{b}_{\mathrm{BC}}-1}
$$

ii) $\quad\left[(12-\mathrm{d}) \cdot \frac{1}{1000 \cdot \eta_{\mathrm{ME}} \cdot \eta_{\mathrm{BH}}} \cdot \beta \cdot \mathrm{TDc}+\mathrm{TCc} \cdot \frac{1}{1000 \cdot \eta_{\mathrm{ME}} \cdot \eta_{\mathrm{BH}}} \cdot \beta \cdot \mathrm{TFd} \cdot \mathrm{NDa}\right]\left(\frac{0,92}{\cos \varphi}-1\right)$

\subsubsection{Sistema com bomba hidráulica acionada por motor elétrico na tarifa conven- cional com o benefício da Portaria 105 do DNAEE (ECI)}

Com base na equação (3.60) tem-se:

$$
\begin{gathered}
\frac{d C A T S^{*}}{d \phi}=0 \Rightarrow \frac{d C F A S^{*}}{d \phi}+\frac{d C A M R *}{d \phi}+ \\
(12-d) \cdot \frac{1}{1000 \cdot \eta_{\mathrm{ME}} \cdot \eta_{\mathrm{BH}}} \cdot \beta \cdot \mathrm{TDc}+0,10 \cdot d \cdot \frac{1}{1000 \cdot \eta_{\mathrm{ME}} \cdot \eta_{\mathrm{BH}}} \cdot \beta \cdot \mathrm{TDc}^{*}+ \\
\text { TCc. } \frac{1}{1000 \cdot \eta_{\mathrm{ME}} \cdot \eta_{\mathrm{BH}}} \cdot \beta \cdot(\mathrm{TFd}-\mathrm{TFdhe} \text { fdtc) }) \mathrm{NDa}=0
\end{gathered}
$$

Caso o sistema não possua banco de capacitores, deve-se excluir da equação (3.67) o termo implícito $i$ e acrescentar no final da mesma o termo $i i$, ou seja, a $\mathrm{dAJ} / \mathrm{d} \phi$ respectiva: 
i) $\frac{1,1 \cdot[\operatorname{tg}(\operatorname{arc} \cos \varphi)-0,402716] \cdot a_{B C} \cdot b_{B C}}{\eta_{\mathrm{BH}}} \cdot \beta \cdot\left(\frac{1,1}{\eta_{\mathrm{BH}}} \cdot \lambda\right)^{\mathrm{B}_{\mathrm{BC}}-1}$
ii) $\left[(12-\mathrm{d}) \frac{1}{1000 \cdot \eta_{\mathrm{ME}} \cdot \eta_{\mathrm{BH}}} \cdot \beta \cdot \mathrm{TDc}+\mathrm{TCc} \cdot \frac{1}{1000 \cdot \eta_{\mathrm{ME}} \cdot \eta_{\mathrm{BH}}} \cdot \beta \cdot(\mathrm{TFd}-\mathrm{TFdhe} \cdot \mathrm{fdtc}) \cdot \mathrm{NDa}\right] \cdot\left(\frac{0,92}{\cos \varphi}-1\right)$

3.4.4. Sistema com bomba hidráulica acionada por motor elétrico na tarifa verde sem o beneficio da Portaria 105 do DNAEE (EVN)

Com base na equação (3.61) tem-se:

$$
\begin{gathered}
\frac{\mathrm{dCATS}^{*}}{\mathrm{~d} \phi}=0 \Rightarrow \frac{\mathrm{dCFAS} *}{\mathrm{~d} \phi}+\frac{\mathrm{dCAMR}{ }^{*}}{\mathrm{~d} \phi}+ \\
(12-\mathrm{d}) \cdot \frac{1}{1000 \cdot \eta_{\mathrm{ME}} \cdot \eta_{\mathrm{BH}}} \cdot \beta \cdot \mathrm{TDV}+0,10 \cdot \mathrm{d} \cdot \frac{1}{1000 \cdot \eta_{\mathrm{ME}} \cdot \eta_{\mathrm{BH}}} \cdot \beta \cdot \mathrm{TDV}^{+}
\end{gathered}
$$

TCvup $\frac{1}{1000 \cdot \eta_{\mathrm{ME}} \cdot \eta_{\mathrm{BH}}} \cdot \beta \cdot$ TFdp. NDu. $\frac{5}{7}+$ TCvufp. $\frac{1}{1000 \cdot \eta_{\mathrm{ME}} \cdot \eta_{\mathrm{BH}}} \cdot \beta \cdot\left(\right.$ TFdfp + TFdp $\left.\cdot \frac{2}{7}\right) \cdot \mathrm{NDu}+$

TCvsp. $\frac{1}{1000 \cdot \eta_{\mathrm{ME}} \cdot \eta_{\mathrm{BH}}} \cdot \beta$. TFdp. NDs. $\frac{5}{7}+$ TCvsfp. $\frac{1}{1000 \cdot \eta_{\mathrm{ME}} \cdot \eta_{\mathrm{BH}}} \cdot \beta \cdot\left(\right.$ TFdfp + TFdp. $\left.\frac{2}{7}\right) \cdot \mathrm{NDs}=0$ (3.68)

Caso o sistema não possua banco de capacitores, deve-se excluir da equação (3.68) o termo implicito $i$ e acrescentar no final da mesma o termo $i i$, ou seja, a $\mathrm{dAJ} / \mathrm{d} \phi$ respectiva:

i)

$$
\frac{1,1 \cdot[\operatorname{tg}(\operatorname{arc} \cos \varphi)-0,402716] \cdot a_{\mathrm{BC}} \cdot b_{\mathrm{BC}}}{\eta_{\mathrm{BH}}} \cdot \beta \cdot\left(\frac{1,1}{\eta_{\mathrm{BH}}} \cdot \lambda\right)^{\mathrm{b}_{\mathrm{BC}}-1}
$$

ii)

$$
\left[(12-d) \cdot \frac{1}{1000 \cdot \eta_{\mathrm{ME}} \cdot \eta_{\mathrm{BH}}} \cdot \beta \cdot \mathrm{TDv}+\mathrm{TCvup} \cdot \frac{1}{1000 \cdot \eta_{\mathrm{ME}} \cdot \eta_{\mathrm{BH}}} \cdot \beta \cdot \mathrm{TFdp} \cdot \mathrm{NDu} \cdot \frac{5}{7}+\right.
$$

TCvufp. $\frac{1}{1000 \cdot \eta_{\mathrm{ME}} \cdot \eta_{\mathrm{BH}}} \cdot \beta \cdot\left(\right.$ TFdfp + TFdp. $\left.\frac{2}{7}\right) \cdot$ NDu + TCvsp. $\frac{1}{1000 \cdot \eta_{\mathrm{ME}} \cdot \eta_{\mathrm{BH}}} \cdot \beta \cdot \operatorname{TFdp} \cdot$ NDs $\frac{5}{7}+$ $\left.\operatorname{TCVsfp} \cdot \frac{1}{1000 \cdot \eta_{\mathrm{ME}} \cdot \eta_{\mathrm{BH}}} \beta \cdot\left(\operatorname{TFdp}+\operatorname{TFdp} \cdot \frac{2}{7}\right) \cdot \mathrm{NDs}\right] \cdot\left(\frac{0,92}{\cos \varphi}-1\right)$

3.4.5. Sistema com bomba hidráulica acionada por motor elétrico na tarifa verde com o beneficio da Portaria 105 do DNAEE (EVI)

Com base na equação (3.62) tem-se: 


$$
\begin{aligned}
& \frac{\mathrm{dCATS}^{*}}{\mathrm{~d} \phi}=0 \Rightarrow \frac{\mathrm{dCFAS}^{*}}{\mathrm{~d} \phi}+\frac{\mathrm{dCAMR}^{*}}{\mathrm{~d} \phi}+ \\
& (12-\mathrm{d}) \cdot \frac{1}{1000 \cdot \eta_{\mathrm{ME}} \cdot \eta_{\mathrm{BH}}} \cdot \beta \cdot \mathrm{TDV}+0,10 \cdot \mathrm{d} \cdot \frac{1}{1000 \cdot \eta_{\mathrm{ME}} \cdot \eta_{\mathrm{BH}}} \cdot \beta \cdot \mathrm{TDV}+ \\
& \text { TCvup. } \frac{1}{1000 \cdot \eta_{\mathrm{ME}} \cdot \eta_{\mathrm{BH}}} \cdot \beta \text {. TFdp. NDu. } \frac{5}{7}+ \\
& \text { TCvufp. } \frac{1}{1000 \cdot \eta_{\mathrm{ME}} \cdot \eta_{\mathrm{BH}}} \cdot \beta \cdot\left[\left(\text { TFdfp }+ \text { TFdp } \cdot \frac{2}{7}\right)-\text { TFdhe. fdtc }\right] \text { NDu }+ \\
& \text { TCvsp. } \frac{1}{1000 \cdot \eta_{\mathrm{ME}} \cdot \eta_{\mathrm{BH}}} \cdot \beta \cdot \text { TFdp.NDs. } \frac{5}{7}+ \\
& \text { TCvsfp. } \frac{1}{1000 \cdot \eta_{\mathrm{ME}} \cdot \eta_{\mathrm{BH}}} \cdot \beta \cdot\left[\left(\operatorname{TFdfp}+\operatorname{TFdp} \cdot \frac{2}{7}\right)-\text { TFdhe. fdtc }\right] \text { NDs }=0
\end{aligned}
$$

Caso o sistema não possua banco de capacitores, deve-se excluir da equação (3.69) o termo implícito $i$ e acrescentar no final da mesma o termo $i i$, ou seja, a dAJ/d $\phi$ respectiva:

i)

$$
\frac{1,1 \cdot[\operatorname{tg}(\operatorname{arc} \cos \varphi)-0,402716] \cdot a_{B C} \cdot b_{B C}}{\eta_{B H}} \cdot \beta \cdot\left(\frac{1,1}{\eta_{B H}} \cdot \lambda\right)^{b_{B C}-1}
$$

ii) $\quad\left[(12-d) \cdot \frac{1}{1000 \cdot \eta_{\mathrm{ME}} \cdot \eta_{\mathrm{BH}}} \cdot \beta \cdot \operatorname{TDv}+\right.$ TCvup $\frac{1}{1000 \cdot \eta_{\mathrm{ME}} \cdot \eta_{\mathrm{BH}}} \cdot \beta \cdot T F d p \cdot N D u \cdot \frac{5}{7}+$ TCvufp. $\frac{1}{1000 \cdot \eta_{\mathrm{ME}} \cdot \eta_{\mathrm{BH}}} \cdot \beta \cdot\left[\left(\right.\right.$ TFdfp + TFdp $\left.\cdot \frac{2}{7}\right)-$ TFdhe.fdtc $] \cdot \mathrm{NDu}+$ TCvsp. $\frac{1}{1000 \cdot \eta_{\mathrm{ME}} \cdot \eta_{\mathrm{BH}}} \cdot \beta$. TFdp.NDs. $\frac{5}{7}+$

TCvsfp. $\frac{1}{1000 \cdot \eta_{\mathrm{ME}} \cdot \eta_{\mathrm{BH}}} \cdot \beta \cdot\left[\left(\operatorname{TFdfp}+\right.\right.$ TFdp. $\left.\frac{2}{7}\right)-$ TFdhe. fdtc $\left.] \cdot \operatorname{NDs}\right] \cdot\left(\frac{0,92}{\cos \varphi}-1\right)$

\subsubsection{Sistema com bomba hidráulica acionada por motor elétrico na tarifa azul sem o benefício da Portaria 105 do DNAEE (EAN)}

Com base na equação (3.64) tem-se:

$$
\begin{gathered}
\frac{\mathrm{dCATS} *}{\mathrm{~d} \phi}=0 \Rightarrow \frac{\mathrm{dCFAS}}{\mathrm{d} \phi}+\frac{\mathrm{dCAMR} *}{\mathrm{~d} \phi}+ \\
(5-\mathrm{dup}) \cdot \frac{1}{1000 \cdot \eta_{\mathrm{ME}} \cdot \eta_{\mathrm{BH}}} \cdot \beta \cdot \text { TDap }+0,10 \cdot \mathrm{dup} \cdot \frac{1}{1000 \cdot \eta_{\mathrm{ME}} \cdot \eta_{\mathrm{BH}}} \cdot \beta \cdot \text { TDap }+ \\
(5-\mathrm{dufp}) \cdot \frac{1}{1000 \cdot \eta_{\mathrm{ME}} \cdot \eta_{\mathrm{BH}}} \cdot \beta \cdot \text { TDafp }+0,10 \cdot \mathrm{dufp} \cdot \frac{1}{1000 \cdot \eta_{\mathrm{ME}} \cdot \eta_{\mathrm{BH}}} \cdot \beta \cdot \text { TDafp }+
\end{gathered}
$$




$$
\begin{aligned}
& (7-\mathrm{dsp}) \cdot \frac{1}{1000 \cdot \eta_{\mathrm{ME}} \cdot \eta_{\mathrm{BH}}} \cdot \beta \cdot \text { TDap }+0,10 \cdot \mathrm{dsp} \cdot \frac{1}{1000 \cdot \eta_{\mathrm{ME}} \cdot \eta_{\mathrm{BH}}} \cdot \beta \cdot \text { TDap }+ \\
& (7-\mathrm{dsfp}) \cdot \frac{1}{1000 \cdot \eta_{\mathrm{ME}} \cdot \eta_{\mathrm{BH}}} \cdot \beta \cdot \text { TDafp }+0,10 \mathrm{dsfp} \cdot \frac{1}{1000 \cdot \eta_{\mathrm{ME}} \cdot \eta_{\mathrm{BH}}} \cdot \beta \cdot \text { TDafp }+
\end{aligned}
$$

TCaup. $\frac{1}{1000 \cdot \eta_{\mathrm{ME}} \cdot \eta_{\mathrm{BH}}} \cdot \beta \cdot \operatorname{TFdp} \cdot$ NDu $\frac{5}{7}+$ TCaufp. $\frac{1}{1000 \cdot \eta_{\mathrm{ME}} \cdot \eta_{\mathrm{BH}}} \cdot \beta \cdot\left(\right.$ TFdfp + TFdp $\left.\frac{2}{7}\right) \cdot \mathrm{NDu}+$ TCasp. $\frac{1}{1000 \cdot \eta_{\mathrm{ME}} \cdot \eta_{\mathrm{BH}}} \cdot \beta \cdot$ TFdp. NDs. $\frac{5}{7}+$ TCasfp. $\frac{1}{1000 \cdot \eta_{\mathrm{ME}} \cdot \eta_{\mathrm{BH}}} \cdot \beta \cdot\left(\operatorname{TFdfp}+\right.$ TFdp $\left.\frac{2}{7}\right) \cdot \mathrm{NDs}=0(3.70)$

Caso o sistema não possua banco de capacitores, deve-se excluir da equação (3.70) ० termo implícito $i$ e acrescentar no final da mesma o termo $i i$, ou seja, a $\mathrm{dAJ} / \mathrm{d} \phi$ respectiva:

$$
\text { i) } \begin{aligned}
& \frac{1,1 \cdot[\operatorname{tg}(\operatorname{arc} \cos \varphi)-0,402716] \cdot a_{\mathrm{BC}} \cdot b_{\mathrm{BC}}}{\eta_{\mathrm{BH}}} \cdot \beta \cdot\left(\frac{1,1}{\eta_{\mathrm{BH}}} \cdot \lambda\right)^{\mathrm{b}_{\mathrm{BC}}-1} \\
& {\left[(5-\mathrm{dup}) \cdot \frac{1}{1000 \cdot \eta_{\mathrm{ME}} \cdot \eta_{\mathrm{BH}}} \cdot \beta \cdot \operatorname{TDap}+(5-\mathrm{dufp}) \cdot \frac{1}{1000 \cdot \eta_{\mathrm{ME}} \cdot \eta_{\mathrm{BH}}} \cdot \beta \cdot \text { TDafp }+\right.} \\
&(7-\mathrm{dsp}) \cdot \frac{1}{1000 \cdot \eta_{\mathrm{ME}} \cdot \eta_{\mathrm{BH}}} \cdot \beta \cdot \mathrm{TDap}+(7-\mathrm{dsfp}) \cdot \frac{1}{1000 \cdot \eta_{\mathrm{ME}} \cdot \eta_{\mathrm{BH}}} \cdot \beta \cdot \text { TDafp }+
\end{aligned}
$$

TCaup. $\frac{1}{1000 \cdot \eta_{\mathrm{ME}} \cdot \eta_{\mathrm{BH}}} \cdot \beta \cdot$ TFdp.NDu. $\frac{5}{7}+$ TCaufp. $\frac{1}{1000 \cdot \eta_{\mathrm{ME}} \cdot \eta_{\mathrm{BH}}} \cdot \beta \cdot\left(\right.$ TFdfp + TFdp. $\left.\frac{2}{7}\right) \cdot \mathrm{NDu}+$ TCasp. $\frac{1}{1000 \cdot \eta_{\mathrm{ME}} \cdot \eta_{\mathrm{BH}}} \cdot \beta \cdot$ TFdp. NDs $\frac{5}{7}+$ TCasfp. $\frac{1}{1000 \cdot \eta_{\mathrm{ME}} \cdot \eta_{\mathrm{BH}}} \cdot \beta \cdot\left(\right.$ TFdfp + TFdp. $\left.\left.\frac{2}{7}\right) \cdot \mathrm{NDs}\right]\left(\frac{0,92}{\cos \varphi}-1\right)$

\subsubsection{Sistema com bomba hidráulica acionada por motor elétrico na tarifa azul com o beneficio da Portaria 105 do DNAEE (EAI)}

Com base na equação (3.64) tem-se:

$$
\begin{aligned}
& \frac{\mathrm{dCATS}^{*}}{\mathrm{~d} \phi}=0 \Rightarrow \frac{\mathrm{dCFAS}^{*}}{\mathrm{~d} \phi}+\frac{\mathrm{dCAMR}^{*}}{\mathrm{~d} \phi}+ \\
& (5-\text { dup }) \cdot \frac{1}{1000 \cdot \eta_{\mathrm{ME}} \cdot \eta_{\mathrm{BH}}} \cdot \beta \cdot \text { TDap }+0,10 \cdot \mathrm{dup} \cdot \frac{1}{1000 \cdot \eta_{\mathrm{ME}} \cdot \eta_{\mathrm{BH}}} \cdot \beta \cdot \text { TDap }+ \\
& (5-\text { dufp }) \cdot \frac{1}{1000 \cdot \eta_{\mathrm{ME}} \cdot \eta_{\mathrm{BH}}} \cdot \beta \cdot \operatorname{TDafp}+0,10 \cdot \text { dufp. } \frac{1}{1000 \cdot \eta_{\mathrm{ME}} \cdot \eta_{\mathrm{BH}}} \cdot \beta \cdot \text { TDafp }+ \\
& (7-\mathrm{dsp}) \cdot \frac{1}{1000 \cdot \eta_{\mathrm{ME}} \cdot \eta_{\mathrm{BH}}} \cdot \beta \cdot \mathrm{TDap}+0,10 \cdot \mathrm{dsp} \cdot \frac{1}{1000 \cdot \eta_{\mathrm{ME}} \cdot \eta_{\mathrm{BH}}} \cdot \beta \cdot \text { TDap }+ \\
& (7-\mathrm{dsfp}) \frac{1}{1000 \cdot \eta_{\mathrm{ME}} \cdot \eta_{\mathrm{BH}}} \cdot \beta \cdot \operatorname{TDafp}+0,10 \cdot \mathrm{dsfp} \cdot \frac{1}{1000 \cdot \eta_{\mathrm{ME}} \cdot \eta_{\mathrm{BH}}} \cdot \beta \cdot \text { TDafp }+
\end{aligned}
$$




$$
\begin{gathered}
\text { TCaup } \frac{1}{1000 \cdot \eta_{\mathrm{ME}} \cdot \eta_{\mathrm{BH}}} \cdot \beta \cdot \mathrm{TFdp} \cdot \mathrm{NDu} \cdot \frac{5}{7}+ \\
\text { TCaufp } \frac{1}{1000 \cdot \eta_{\mathrm{ME}} \cdot \eta_{\mathrm{BH}}} \cdot \beta \cdot\left[\left(\mathrm{TFdfp}+\mathrm{TFdp} \cdot \frac{2}{7}\right)-\mathrm{TFdhe} \cdot \mathrm{fdtc}\right] \mathrm{NDu}+ \\
\text { TCasp } \cdot \frac{1}{1000 \cdot \eta_{\mathrm{ME}} \cdot \eta_{\mathrm{BH}}} \cdot \beta \cdot \mathrm{TFdp} \cdot \mathrm{NDs} \cdot \frac{5}{7}+ \\
\text { TCasfp. } \frac{1}{1000 \cdot \eta_{\mathrm{ME}} \cdot \eta_{\mathrm{BH}}} \cdot \beta \cdot\left[\left(\mathrm{TFdfp}+\mathrm{TFdp} \cdot \frac{2}{7}\right)-\text { TFdhe. fdtc }\right] \text { NDs }=0
\end{gathered}
$$

Caso o sistema não possua banco de capacitores, deve-se excluir da equação (3.71) o termo implicito $i$ e acrescentar no final da mesma o termo $i i$, ou seja, a $\mathrm{dAJ} / \mathrm{d} \phi$ respectiva:

i)

$$
\begin{aligned}
& \frac{1,1 \cdot[\operatorname{tg}(\operatorname{arc} \cos \varphi)-0,402716] \cdot a_{B C} \cdot b_{B C}}{\eta_{\mathrm{BH}}} \cdot \beta \cdot\left(\frac{1,1}{\eta_{\mathrm{BH}}} \cdot \lambda\right)^{\mathrm{b}_{\mathrm{BC}}-1} \\
& \text { ii) } \quad\left[(5-\text { dup }) \cdot \frac{1}{1000 \cdot \eta_{\mathrm{ME}} \cdot \eta_{\mathrm{BH}}} \cdot \beta \cdot \text { TDap }+(5-\mathrm{dufp}) \cdot \frac{1}{1000 \cdot \eta_{\mathrm{ME}} \cdot \eta_{\mathrm{BH}}} \cdot \beta \cdot \text { TDafp }+\right. \\
& (7-\mathrm{dsp}) \cdot \frac{1}{1000 \cdot \eta_{\mathrm{ME}} \cdot \eta_{\mathrm{BH}}} \cdot \beta \cdot \text { TDap }+(7-\mathrm{dsfp}) \cdot \frac{1}{1000 \cdot \eta_{\mathrm{ME}} \cdot \eta_{\mathrm{BH}}} \cdot \beta \cdot \text { TDafp }+ \\
& \text { TCaup } \frac{1}{1000 \cdot \eta_{\mathrm{ME}} \cdot \eta_{\mathrm{BH}}} \cdot \beta . \text { TFdp. NDu. } \frac{5}{7}+ \\
& \text { TCaufp } \cdot \frac{1}{1000 \cdot \eta_{\mathrm{ME}} \cdot \eta_{\mathrm{BH}}} \cdot \beta \cdot\left[\left(\text { TFdfp }+ \text { TFdp } \cdot \frac{2}{7}\right)-\text { TFdhe. fdtc }\right] \text { NDu }+ \\
& \text { TCasp } \frac{1}{1000 \cdot \eta_{\mathrm{ME}} \cdot \eta_{\mathrm{BH}}} \cdot \beta . \text { TFdp. NDs. } \frac{5}{7}+ \\
& \text { TCasfp. } \left.\frac{1}{1000 \cdot \eta_{\mathrm{ME}} \cdot \eta_{\mathrm{BH}}} \cdot \beta \cdot\left[\left(\operatorname{TFdfp}+\text { TFdp } \frac{2}{7}\right)-\text { TFdhe.fdtc }\right] \cdot \text { NDs }\right] \cdot\left(\frac{0,92}{\cos \varphi}-1\right)
\end{aligned}
$$




\section{APLICAÇÃO E DISCUSSÃO}

O modelo desenvolvido no item 3 foi aplicado, através do programa anexo na contracapa deste trabalho, para calcular o diâmetro econômico e estimar os custos de um sistema de recalque de um equipamento de irrigação do tipo pivô central e para verificar a sensibilidade do mesmo diante da variação do comprimento da linha de alta tensão. Os dados do sistema e das condições locais foram obtidos da Fazenda Santa Clarisse, Pereira Barreto (SP). Os preços dos equipamentos, construção, combustível e tarifas de energia elétrica foram obtidos entre setembro de 1996 e fevereiro de 1997, período que os mesmos mantiveram-se relativamente estáveis.

Valores dos parâmetros conhecidos, utilizados em todas opções do sistema de recalque (D, ECN, ECI, EVN, EVI, EAN e EAI):

$R$ - fração do custo do sistema novo após o periodo de depreciação: 0,10 ;

PA - periodo de depreciação: 10 anos;

r - taxa de juros anual: 0,065 (6,5\%);

Ls - comprimento da linha de sucção: $12 \mathrm{~m}$;

Vs - velocidade de escoamento na tubulação de sucção (diâmetro $300 \mathrm{~mm}$ ): 0,9635 m/s;

$\mathrm{Lr}$ - comprimento da linha de recalque: $552 \mathrm{~m}$;

$\mathrm{Lu}$ - comprimento de um tubo (aço galvanizado): $6 \mathrm{~m}$;

$\mathrm{Cr}=\mathrm{Cs}$ - coeficiente de rugosidade médio da fórmula de Hazen-Williams dos tubos do recalque e sucção, respectivamente, para o aço galvanizado: 115 ;

$\mathrm{Q}$ - vazão do sistema: $0,06811 \mathrm{~m}^{3} / \mathrm{s}\left(245,19 \mathrm{~m}^{3} / \mathrm{h}\right)$;

HR - altura de recalque: $21 \mathrm{~m}$;

PF - carga manométrica necessária no final da adutora: $48,85 \mathrm{~m}$;

$\eta_{\mathrm{BH}}$ - rendimento esperado da bomba hidráulica: 0,78 (valor médio obtido das curvas características dos três modelos mais prováveis). Em caso de dúvida, sugere-se o valor 0,71 , que é o valor médio de 56 amostras para potências de $345 \mathrm{~W}$ a $109184 \mathrm{~W}$, com coeficiente de variação igual a $16,38 \%$; 
$\mathrm{ACB}$ - área da casa de bomba: $14 \mathrm{~m}^{2}$;

PCC - custo da construção civil para obras de acabamento médio, considerado $150,00 \mathrm{US} \$ / \mathrm{m}^{2}$;

DV - profundidade da vala de assentamento da tubulação da adutora: $1,30 \mathrm{~m}$;

WV - largura da vala de assentamento da tubulação adutora: $1,00 \mathrm{~m}$;

PVE - custo do metro cúbico de escavação, considerado $3,71 \mathrm{US} \$ / \mathrm{m}^{3}$.

Acessórios:

- Curva de $60^{\circ}: \mathrm{n}=2$ (recalque); $\mathrm{K}=0,30$

- Curva de $90^{\circ}: \mathrm{n}=3$ (1 para a linha de sucção e 2 para a de recalque); $\mathrm{K}=0,40$;

- Luva cônica concêntrica (ampliação): $\mathbf{n}=1$ (recalque); $\mathrm{K}=0,30$;

- Luva cônica excêntrica (redução): $n=1$ (sucção); $K=0,15$;

- Registro de gaveta: $n=1 ; K=0,20$;

- Válvula de pé com crivos para sucção: $n=1 ; K=1,75$;

- Válvula de retenção flangeada com "by-pass": $n=1 ; K=2,50$.

Através da metodologia proposta no item 3.1.1, que trata da estimativa de custos dos equipamentos novos utilizados nos sistemas de recalque, foram obtidos os coeficientes de ajuste da regressão, apresentados na Tabela 6 .

Tabela 6. Valores dos coeficientes $a_{i}$ e $b_{i}$ da equação (3.4), do NMS (nível mínimo de significância da regressão) e do $\mathbf{R}^{2}$ (coeficiente de determinação da regressão).

\begin{tabular}{lcccc}
\hline \multicolumn{1}{c}{ EQUIPAMENTO } & $\mathbf{a}_{\mathbf{i}}$ & $\mathbf{b}_{\mathbf{i}}$ & NMS & $\mathbf{R}^{\mathbf{2}}$ \\
\hline Banco de capacitores - imerso em óleo - 380 V & 0,33703 & 0,68571 & $1,79 \mathrm{E}-7$ & 0,972 \\
Bomba hidráulica - 1750 rpm & 92,3939 & 0,42822 & $1,35 \mathrm{E}-7$ & 0,944 \\
Chave de partida compensadora - 380 Volts & 2,97729 & 0,67556 & $1,35 \mathrm{E}-6$ & 0,953 \\
Curva 60 graus - aço galvanizado flangeado (AGF) & 203,90 & 0,92777 & $6,88 \mathrm{E}-4$ & 0,872 \\
Curva 90 graus - AGF & 394,54 & 0,94950 & $2,86 \mathrm{E}-4$ & 0,904 \\
Luva cônica concêntrica - AGF & 345,43 & 1,40135 & $8,33 \mathrm{E}-7$ & 0,958 \\
Luva cônica excêntrica - AGF & 507,09 & 1,25321 & $2,55 \mathrm{E}-8$ & 0,960 \\
Motor diesel completo p/ bombeamento & 38,6849 & 0,60017 & $7,24 \mathrm{E}-7$ & 0,942 \\
Motor elétrico trifásico - IP 54 - 4 pólos & 0,099151 & 0,92728 & $2,36 \mathrm{E}-11$ & 0,997 \\
Transformador elétrico trifásico - classe 15kV & 1,287676 & 0,66242 & $1,05 \mathrm{E}-6$ & 0,985 \\
Tubo de 6 metros - AGF & 1034,7 & 1,36696 & $2,17 \mathrm{E}-10$ & 0,990 \\
Válvula de gaveta fofo / bronze - AGF & 8536,10 & 1,35975 & $2,98 \mathrm{E}-6$ & 0,943 \\
Válvula de retenção com "by-pass" - AGF & 843,75 & 0,62079 & $3,00 \mathrm{E}-6$ & 0,979 \\
Válvula de sucção - AGF & 1100,94 & 1,33285 & $6,17 \mathrm{E}-5$ & 0,942 \\
\hline \hline
\end{tabular}


Conforme a Tabela 6 , pode-se verificar, pelo nível mínimo de significância e pelo coeficiente de determinação da regressão, que o modelo multiplicativo ajusta-se adequadamente aos dados dos equipamentos hidráulicos e elétricos, que podem ser observados no Apêndice 1 .

\subsection{Diâmetro da tubulação de recalque que minimiza o custo anual total do sistema de recalque}

O preço do combustivel e das tarifas de energia elétrica (nas diversas modalidades e com os descontos especiais concedidos para os consumidores rurais irrigantes exclusivos) utilizados neste item estão apresentados no Apêndice 2. 0 tempo de funcionamento anual do sistema (TFa) foi fixado em $2400 \mathrm{~h}$, sendo este valor igual para todas as opções do sistema, para que as comparações sejam feitas com eqüidade.

$\mathrm{O}$ diâmetro econômico da tubulação de recalque $\left(\phi_{\mathrm{e}}\right)$ do sistema de recalque com bomba hidráulica acionada por motor à combustão (D) foi calculado substituindo-se, além dos parâmetros já conhecidos, os seguintes parâmetros na equação (3.65):

$\mathrm{CC}_{\mathrm{u}}$ - consumo médio de combustível (diesel) do motor por unidade de potência útil por hora: $3,020408 \times 10^{-7} \mathrm{~m}^{3} / \mathrm{W} . \mathrm{h}$ (Tabela de equivalência de consumo: motores diesel versus motores elétricos - CESP / CPFL / ELETROPAULO);

TFa - tempo de funcionamento anual do sistema: $2400 \mathrm{~h}$;

Para o cálculo do $\phi_{\mathrm{e}}$ do sistema de recalque com bomba hidráulica acionada por motor à eletricidade em cada modalidade de tarifação da energia elétrica (ECN, ECI, EVN, EVI, EAN e EAI), foram considerados também os seguintes parâmetros comuns a todas elas:

PMLAT - custo médio da linha de alta tensão com postes de eucalipto tratados ( 9 e $10 \mathrm{~m}$ ) e cabos

CAA - 4 AWG $\left(21,2 \mathrm{~mm}^{2}\right)$, cujo valor para a Companhia Paulista de Força e Luz CPFL - è 7,60 US $\$ / \mathrm{m}$;

LLAT - comprimento da linha de alta tensão: $1800 \mathrm{~m}$;

$\eta_{\mathrm{ME}}$ - rendimento do motor elétrico: 0,88 (valor médio de 24 amostras de motor 4 pólos - $60 \mathrm{~Hz}$, para potências de $3675 \mathrm{~W}$ a $367500 \mathrm{~W}$, com coeficiente de variação igual a 4,86\%);

$\cos \varphi$ - fator de potência do motor elétrico: 0,87 (valor médio de 24 amostras de motor 4 pólos $60 \mathrm{~Hz}$, para potências de $3675 \mathrm{~W}$ a $367500 \mathrm{~W}$, com coeficiente de variação de $2,51 \%$ ); 
$\operatorname{arc} \cos \varphi=\operatorname{arc} \cos 0,87$ - arco correspondente à defasagem entre corrente e tensão no circuito elétrico do sistema sem banco de capacitores, cujo valor é 0,515594 rad. Com isso, o termo entre colchetes da equação (3.19) é 0,1407279 ;

Obs: considerou-se a presença do banco de capacitores na aplicação do modelo em todas opções do sistema à eletricidade.

O valor do $\phi_{\mathrm{e}}$ do sistema ECN foi calculado substituindo-se, além dos parâmetros já conhecidos, os seguintes parâmetros na equação (3.66):

$\mathrm{d}$ - número de meses completos por ano que o sistema de recalque fica desligado: 4;

TFd - tempo médio de funcionamento diário do sistema de recalque: $20 \mathrm{~h}$;

$\mathrm{NDa}$ - número de dias por ano de funcionamento do sistema de recalque: 120.

$\mathrm{O}$ valor do $\phi_{\mathrm{e}}$ do sistema $\mathrm{ECI}$ foi calculado substituindo-se, além dos parâmetros já conhecidos, os seguintes parâmetros na equação (3.67):

$\mathrm{d}$ - número de meses completos por ano que o sistema de recalque fica desligado: 4;

TFdhe - tempo médio de funcionamento diário do sistema de recalque no horário especial para irrigante: $6 \mathrm{~h}$;

TFdhc - tempo médio de funcionamento diário do sistema de recalque no horário complementar ao horário especial para irrigante: $14 \mathrm{~h}$;

$\mathrm{NDa}$ - número de dias por ano de funcionamento do sistema de recalque: 120;

fdtc - fração de desconto da tarifa de consumo de energia elétrica conforme a Portaria 105 do DNAEE: 0,70 .

O valor do $\phi_{\mathrm{e}}$ do sistema EVN foi calculado substituindo-se, além dos parâmetros já conhecidos, os seguintes parâmetros na equação (3.68):

$\mathrm{d}$ - número de meses completos por ano que o sistema de recalque fica desligado: 4;

TFdp - tempo médio de funcionamento diário do sistema de recalque no horário de ponta: $1 \mathrm{~h}$;

TFdfp - tempo médio de funcionamento diário do sistema de recalque no horário fora de ponta: $19 \mathrm{~h}$;

NDs - número de dias de funcionamento do sistema de recalque no período seco do ano: 90

$\mathrm{NDu}$ - número de dias de funcionamento do sistema de recalque no periodo úmido do ano: 30 .

O valor do $\phi_{\mathrm{e}}$ do sistema EVI foi calculado substituindo-se, além dos parâmetros já conhecidos, os seguintes parâmetros na equação (3.69): 
$\mathrm{d}$ - número de meses completos por ano que o sistema de recalque fica desligado: 4 ;

TFdp - tempo médio de funcionamento diário do sistema de recalque no horário de ponta: $1 \mathrm{~h}$;

TFdfp - tempo médio de funcionamento diário do sistema de recalque no horário fora de ponta: $19 \mathrm{~h}$ (neste valor está incluído o TFdhe);

TFdhe - tempo médio de funcionamento diário do sistema de recalque no horário especial para irrigantes: $6 \mathrm{~h}$;

NDs - número de dias de funcionamento do sistema de recalque no período seco do ano: 90

$\mathrm{NDu}$ - número de dias de funcionamento do sistema de recalque no periodo úmido do ano: 30 ;

fatc - fração de desconto da tarifa de consumo de energia elétrica conforme a Portaria 105 do DNAEE: 0,70 .

O valor do $\phi_{\mathrm{e}}$ do sistema EAN foi calculado substituindo-se, além dos parâmetros já conhecidos, os seguintes parâmetros na equação (3.70):

dsfp - número de meses completos que o sistema de recalque fica desligado no período seco do ano no horário fora de ponta: 1 ;

dsp - número de meses completos que o sistema de recalque fica desligado no período seco do ano no horário de ponta: 1 ;

dufp - número de meses completos que o sistema de recalque fica desligado no período úmido do ano no horário fora de ponta: 3 ;

dup - número de meses completos que o sistema de recalque fica desligado no período úmido do ano no horário de ponta: 3 ;

TFdp - tempo médio de funcionamento diário do sistema de recalque no horário de ponta: $1 \mathrm{~h}$;

TFdfp - tempo médio de funcionamento diário do sistema de recalque no horário fora de ponta: $19 \mathrm{~h}$;

NDs - número de dias de funcionamento do sistema de recalque no período seco do ano: 90

$\mathrm{NDu}$ - número de dias de funcionamento do sistema de recalque no período úmido do ano: 30 .

O valor do $\phi_{\mathrm{e}}$ do sistema EAI foi calculado substituindo-se, além dos parâmetros já conhecidos, os seguintes parâmetros na equação (3.71):

dsfp - número de meses completos que o sistema de recalque fica desligado no periodo seco do ano no horário fora de ponta: 1 ;

dsp - número de meses completos que o sistema de recalque fica desligado no período seco do ano no horário de ponta: 1 ; 
dufp - número de meses completos que o sistema de recalque fica desligado no período úmido do ano no horário fora de ponta: 3 ;

dup - número de meses completos que o sistema de recalque fica desligado no período úmido do ano no horário de ponta: 3 ;

TFdp - tempo médio de funcionamento diário do sistema de recalque no horário de ponta: $1 \mathrm{~h}$;

TFdfp - tempo médio de funcionamento diário do sistema de recalque no horário fora de ponta:

$19 \mathrm{~h}$ (neste valor está incluído o TFdhe);

TFdhe - tempo médio de funcionamento diário do sistema de recalque no horário especial para irrigantes: $6 \mathrm{~h}$;

NDs - número de dias de funcionamento do sistema de recalque no período seco do ano: 90

$\mathrm{NDu}$ - número de dias de funcionamento do sistema de recalque no período úmido do ano: 30 ;

fdtc - fração de desconto da tarifa de consumo de energia elétrica conforme a Portaria 105 do DNAEE: 0,70 .

A Tabela 7 apresenta os resultados do diâmetro econômico $\left(\phi_{\mathrm{e}}\right)$ referentes à aplicação das equações (3.65), (3.66), (3.67), (3.68), (3.69), (3.70) e (3.71). Também apresenta os resultados do diâmetro comercial mais econômico $\left(\phi_{c e}\right)$, que foi obtido pela substituição dos diâmetros comerciais imediatamente superior e inferior ao teórico $\left(\phi_{\mathrm{e}}\right)$ nas respectivas equações, sendo selecionado aquele que provocou o menor valor nas mesmas.

Tabela 7. Diâmetro econômico $(\mathrm{mm})$ teórico e comercial para cada opção do sistema.

\begin{tabular}{ccccccccc}
\hline Diâmetro & & & \multicolumn{7}{c}{ Opcão do sistema } & & \\
\cline { 2 - 8 } económico & D & ECN & ECI & EVN & EVI & EAN & EAI \\
\hline teórico & 300,4 & 281,2 & 272,5 & 270,2 & 265,5 & 286,5 & 283,0 \\
comercial & 300 & 300 & 250 & 250 & 250 & 300 & 300 \\
\hline \hline
\end{tabular}

Os valores do diâmetro econômico teórico da Tabela 7 revelam que o efeito do custo de bombeamento (CABO) é mais intenso no sistema diesel, vindo em seguida o sistema elétrico azul normal, azul irrigante, convencional normal, convencional irrigante, verde normal e verde irrigante. É necessário também frisar que esta ordem é válida para os dados utilizados nesta aplicação. As justificativas para este comportamento são discutidas com base nos resultados discriminados dos custos do sistema do item seguinte. 


\subsection{Custos do sistema de recalque com bomba hidráulica acionada por motor à combustão e motor à eletricidade nas diversas modalidades de tarifação}

Nos dados técnicos do pivô central instalado na Fazenda Santa Clarisse, que podem ser vistos no Apêndice 3, verifica-se que adutora possui um segmento de $252 \mathrm{~m}$ de comprimento com diâmetro de $250 \mathrm{~mm}$ e outro de $300 \mathrm{~m}$ de comprimento com diâmetro de $200 \mathrm{~mm}$, ambos de aço zincado. Também pode-se verificar que a bomba hidráulica instalada é de marca diferente da utilizada na aplicação do modelo para estimativa dos custos. Porém, para aferição do mesmo, considerou-se em todas as situações a marca da bomba ajustada pela função multiplicativa. Sendo assim, identificaram-se os custos do sistema pelos seguintes tipos:

i) Comercial/Fabricante: custos do sistema para os diâmetros da tubulação de recalque determinados pelo fabricante, sendo considerados os diâmetros e potências comerciais dos equipamentos hidráulicos e elétricos/diesel, respectivamente;

ii) Teórico/Econômico: custos do sistema estimados pelo modelo na condição mais econômica, sendo considerado o diâmetro e potência teórica de cada equipamento hidráulico e elétrico/diesel. Com isso, para cada sistema (op̧̧ão) o CATS foi obtido pela aplicação do valor do diâmetro econômico teórico da Tabela 7 nas equações (3.58), (3.59), (3.60), (3.61), (3.62), (3.63) e (3.64), respectivamente;

iii) Comercial/Teórico/Econômico: custos do sistema estimado pelo modelo utilizando o diâmetro comercial da tubulação de recalque em que ocorre a condição mais econômica, sendo considerado para os demais equipamentos hidráulicos e elétrico/diesel o diâmetro ou a potência teórica. Com isso, para cada sistema (opção) o CATS foi obtido pela aplicação do valor do diâmetro econômico comercial da Tabela 7 nas equações (3.58), (3.59), (3.60), (3.61), (3.62), (3.63) e (3.64), respectivamente;

iv) Comercial/Econômico: custos do sistema utilizancio o diâmetro comercial da tubulação de recalque em que ocorre a condição mais econômica, sendo também considerado o diâmetro e potência comercial de cada equipamento hidráulico e elétrico/diesel. 
A comparação entre os custos do tipo Teórico/Econômico e Comercial/Econômico, principalmente, é que permite verificar melhor a precisão entre o modelo proposto e a situação real econômica. Os Apêndices 4, 5, 6, 7, 8, 9 e 10 apresentam, detalhadamente, os custos CSN, CFAS, CABO, CAMR e CATS dos quatro tipos identificados nas opções do sistema D, ECN, ECI, EVN, EVI, EAN e EAI, respectivamente. Também é apresentada a composição do sistema, ou seja, a discriminação dos equipamentos e estruturas em cada tipo e opção.

Em relação ao custo de aquisição, ou seja, o custo do sistema novo (CSN) e para os dados utilizados na aplicação, a Tabela 8 e a Figura 2 mostram que:

i) no sistema $\mathrm{D}$, o tipo de custo determinado pelo dimensionamento do fabricante (Comercial/Fabricante - C/F) é menor do que os tipos estimados pelo modelo (Teórico/Econômico - T/E; e Comercial/Teórico/Econômico - C/T/E) e o determinado pela condição econômica comercial (Comercial/Econômico - C/E). Isto se deve ao menor custo da tubulação de recalque do primeiro, que não é suficientemente compensado pelo maior custo da bomba hidráulica, motor diesel e acessórios, conforme pode ser verificado no item 1.1 do Apêndice 4. Entre o tipo T/E e o $\mathrm{C} / \mathrm{E}$, verifica-se que o primeiro subestima em $3,1 \%$ o valor do segundo, o que é devido à pequena diferença entre o diâmetro teórico $(300,4 \mathrm{~mm})$ e o comercial $(300,0 \mathrm{~mm})$, bem como aos elevados coeficientes de ajuste das regressões dos equipamentos $\left(R^{2}\right)$;

ii) nos sistemas ECN, EAN e EAI, o tipo C/F é inferior aos tipos T/E, C/T/E e C/E, fato que ocorre de modo inverso nos sistemas ECI, EVN e EVI. No primeiro caso, o custo da tubulação de recalque é menor no tipo $\mathrm{C} / \mathrm{F}$, em relação aos demais, e não é suficientemente compensado pelo maior custo da instalação de bombeamento (bomba e equipamentos elétricos, exclusive a linha de alta tensão), conforme pode ser verificado no item 1.1 dos Apêndices 5, 9 e 10 . Já no segundo caso, embora o custo da tubulação de recalque continue menor no tipo $\mathrm{C} / \mathrm{F}$, o maior custo da instalação de bombeamento mais que compensa esta diferença, conforme pode ser verificado no item 1.1 dos Apêndices 6, 7 e 8;

iii) em relação à precisão do modelo, verifica-se que, em média, o tipo T/E subestima em $0,32 \%$ o valor do tipo $\mathrm{C} / \mathrm{E}$ nos sistemas elétricos. Nos sistemas ECN, EAN e EAI o tipo T/E subestima o valor do $\mathrm{C} / \mathrm{E}(3,1 \%$, em média) porque o diâmetro teórico econômico da tubulação de recalque é menor que o comercial econômico e a diferença de custos da tubulação predomina sobre a diferença de custos da instalação de bombeamento, além do que a condição econômica comercial obriga, em termos médios, a aquisição de equipamentos com diâmetro ou potência imediatamente superior à econômica teórica. Nos sistemas ECI, EVN e EVI o tipo T/E 
estima o valor do $\mathrm{C} / \mathrm{E}$ (2,7\%, em média) porque o diâmetro teórico econômico da tubulação de recalque é maior que o comercial econômico e, novamente, a diferença de custos da tubulação predomina sobre a diferença de custos da instalação de bombeamento.

Tabela 8. Valores do custo do sistema novo (CSN), em US\$, dos tipos Comercial/Fabricante (C/F), Teórico/Econômico (T/E), Comercial/Teórico/Econômico (C/T/E) e Comercial/Econômico (C/E) nas opções do sistema.

\begin{tabular}{cccccccc}
\hline \hline \multirow{2}{*}{$\begin{array}{c}\text { Tipo de } \\
\text { Custo }\end{array}$} & D & ECN & ECI & EVN & EVI & EAN & EAI \\
\cline { 2 - 8 } & $62.646,12$ & $59.623,59$ & $59.623,59$ & $59.623,59$ & $59.623,59$ & $59.623,59$ & $59.623,59$ \\
Com./Fabr. & 62 Opc̃o do sistema \\
Teór./Econ. & $66.464,57$ & $60.004,41$ & $59.306,47$ & $59.129,96$ & $58.766,73$ & $60.436,63$ & $60.152,93$ \\
Com/Teor/Econ & $66.430,18$ & $61.584,63$ & $57.649,31$ & $57.649,31$ & $57.649,31$ & $61.548,63$ & $61.584,63$ \\
Com./Econ. & $68.597,42$ & $62.114,61$ & $57.531,31$ & $57.531,31$ & $57.531,31$ & $62.114,61$ & $62.114,61$ \\
\hline \hline
\end{tabular}

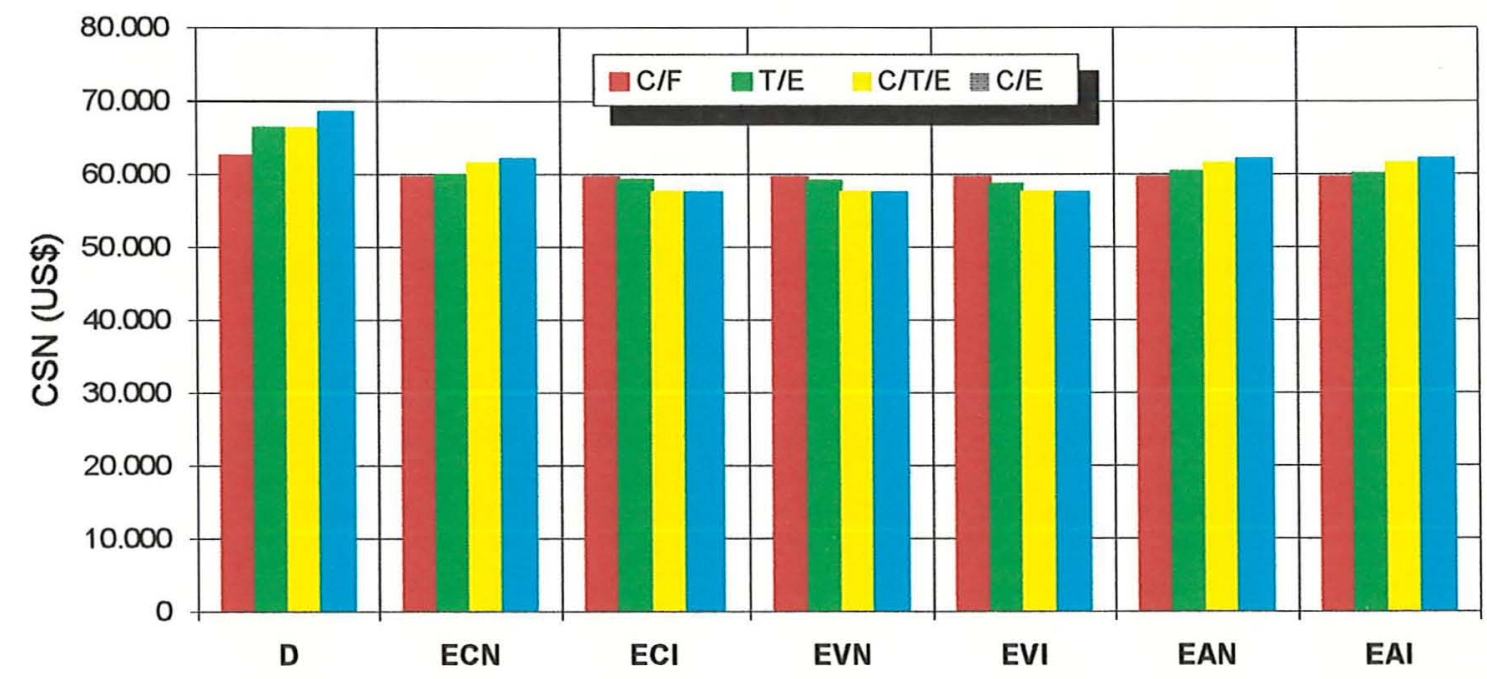

Figura 2. Comportamento do custo do sistema novo (CSN) dos tipos Comercial/Fabricante (C/F), Teórico/Econômico (T/E), Comercial/Teórico/Econômico (C/T/E) e Comercial/Econômico (C/E) nas opções do sistema.

O custo fixo anual do sistema (CFAS), cujos valores encontram-se na Tabela 9, apresenta, para os tipos propostos, o mesmo comportamento que o custo de aquisição, pois estão linearmente relacionados. 
Tabela 9. Valores do custo fixo anual do sistema (CFAS), em US\$, dos tipos Comercial/Fabricante (C/F), Teórico/Econômico (T/E), Comercial/Teórico/Econômico (C/T/E) e Comercial/Econômico (C/E) nas opções do sistema.

\begin{tabular}{cccccccc}
\hline \multirow{2}{*}{ Tipo de } & \multicolumn{7}{c}{ Opcão do sistema } \\
\cline { 2 - 8 } Custo & D & ECN & ECI & EVN & EVI & EAN & EAI \\
\hline Com./Fabr. & $6.417,73$ & $6.108,09$ & $6.108,09$ & $6.108,09$ & $6.108,09$ & $6.108,09$ & $6.108,09$ \\
Teór./Econ. & $6.808,91$ & $6.147,11$ & $6.075,60$ & $6.057,52$ & $6.020,31$ & $6.191,38$ & $6.612,32$ \\
Com/Teor/Econ & $6.805,39$ & $6.308,99$ & $5.905,84$ & $5.905,84$ & $5.905,84$ & $6.308,99$ & $6.308,99$ \\
Com./Econ. & $7.025,57$ & $6.363,28$ & $5.893,75$ & $5.893,75$ & $5.893,75$ & $6.363,28$ & $6.363,28$ \\
\hline \hline
\end{tabular}

Em relação ao custo anual com manutenção e reparos (CAMR), a Tabela 10 e a Figura 3 mostram que seu comportamento é semelhante ao do CSN, pois está diretamente relacionado a este e ao número de horas de funcionamento do sistema no ano, que é igual para todas as opções. Porém, verifica-se o valor expressivamente superior deste custo para o sistema D em relação aos elétricos, o que é devido ao maior custo de manutenção e reparos do motor e acessórios diesel em relação aos equipamentos elétricos. Também deve-se destacar que no sistema D o tipo $\mathrm{C} / \mathrm{F}$ apresenta valor maior que o $\mathrm{T} / \mathrm{E}$ e o $\mathrm{C} / \mathrm{T} / \mathrm{E}$, embora o valor do $\mathrm{CSN}$ seja cerca de $6 \%$ menor, o que pode ser explicado pelo custo maior de seu motor diesel e acessórios e, com isso, gastos com manutenção e reparos, conforme pode ser visto no item 1.1 do Apêndice 4.

Tabela 10. Valores do custo anual com manutenção e reparos (CAMR), em US\$, dos tipos Comercial/Fabricante (C/F), Teórico/Econômico (T/E), Comercial/Teórico/Econômico (C/T/E) e Comercial/Econômico (C/E) nas opções do sistema.

\begin{tabular}{ccccccccc}
\hline Tipo de & \multicolumn{7}{c}{ Opcão do sistema } \\
\cline { 2 - 8 } Custo & D & ECN & ECI & EVN & EVI & EAN & EAI \\
\hline Com./Fabr. & $3.169,56$ & 892,68 & 892,68 & 892,68 & 892,68 & 892,68 & $\mathbf{8 9 2 , 6 8}$ \\
Teór./Econ. & $3.008,14$ & 809,35 & 806,76 & 806,17 & 805,04 & 811,10 & 809,94 \\
Com/Teor/Econ & $3.008,27$ & 816,20 & 802,63 & 802,63 & 802,63 & 816,20 & 816,20 \\
Com./Econ. & $3.205,15$ & 880,83 & $\mathbf{8 5 3 , 3 3}$ & $\mathbf{8 5 3 , 3 3}$ & $\mathbf{8 5 3 , 3 3}$ & $\mathbf{8 8 0 , 8 3}$ & $\mathbf{8 8 0 , 8 3}$ \\
\hline
\end{tabular}




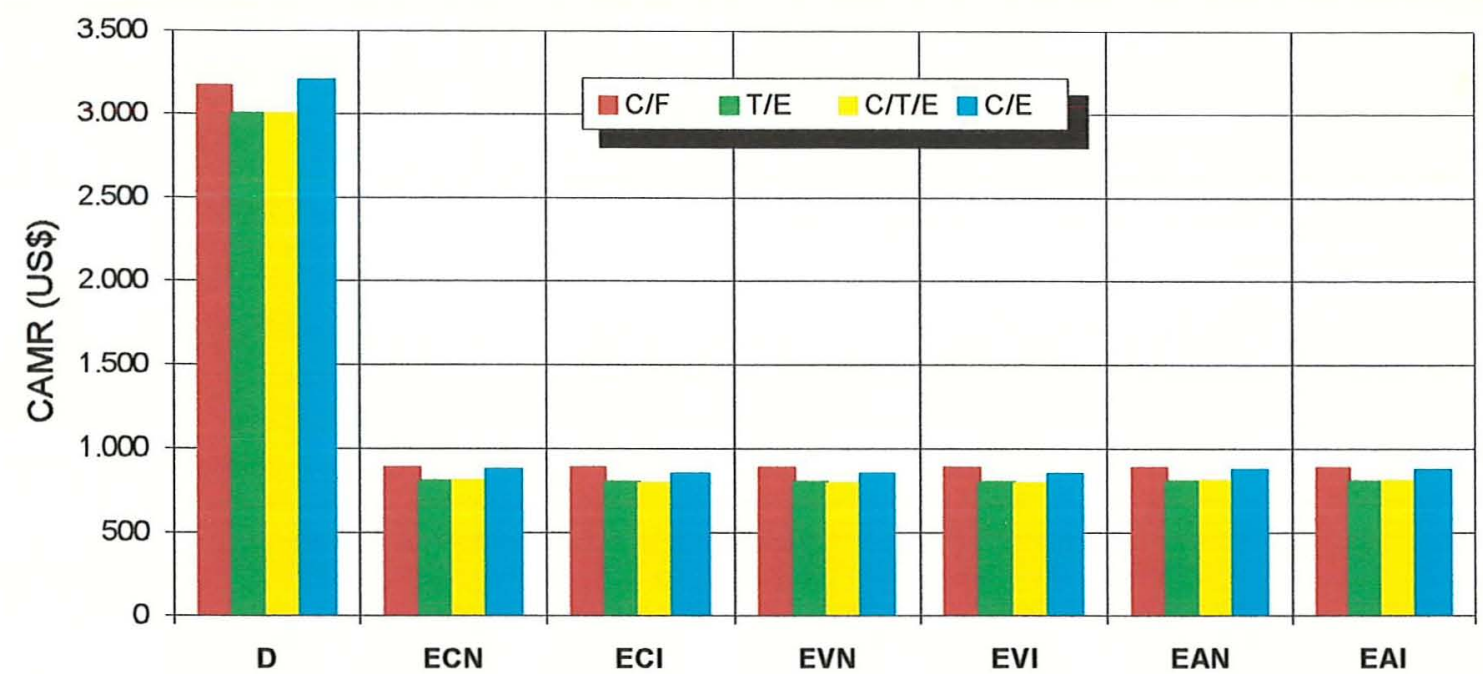

Figura 3. Comportamento do custo anual com manutenção e reparos do sistema (CSN) dos tipos Comercial/Fabricante (C/F), Teórico/Econômico (T/E), Comercial/Teórico/Econômico (C/T/E) e Comercial/Econômico (C/E) nas opções do sistema.

Em relação ao custo anual de bombeamento (CABO) e para os dados utilizados na aplicação, a Tabela 11 e a Figura 4 mostram que:

i) os maiores valores em todos os tipos ocorrem no sistema D, o que explica o fato do diâmetro econômico (teórico e comercial) calculado pelo modelo, neste sistema, ser superior aos dos sistemas à eletricidade, uma vez que o maior efeito deste custo sobre os demais (CFAS e CAMR) "arrasta" o ponto de mínimo do CATS para mais longe da origem dos eixos cartesianos, seja tanto no aumento do CATS (eixo y) quanto no aumento do diâmetro (eixo x);

ii) em todos sistemas, o tipo $\mathrm{C} / \mathrm{F}$ apresenta os maiores valores devido ao menor diâmetro médio da tubulação de recalque, o que acarreta maiores "perdas de carga" no escoamento e, portanto, maiores exigências de potência da bomba hidráulica e de energia para seu acionamento;

iii) nos sistemas à eletricidade, os valores do tipo $\mathrm{T} / \mathrm{E}$ e $\mathrm{C} / \mathrm{T} / \mathrm{E}$ são um pouco maiores que o tipo $\mathrm{C} / \mathrm{E}$ porque nos primeiros o rendimento do motor elétrico (que fornece à bomba hidráulica somente a potência necessária) é considerado $88 \%$ (modelo), enquanto no segundo é $91 \%$ (dado real segundo o fabricante de motores), conforme pode ser visto no item 2 dos Apêndices 5, 6, 7, 8, 9 e 10. Este melhor rendimento compensa a maior "perda de carga" ocorrida nos sistemas ECI, EVN e EVI, quando são comparados os tipos T/E e C/E;

iv) nos sistemas elétricos, o valor decresceu para os irrigantes, dentro das respectivas modalidades, devido ao desconto concedido no consumo de energia elétrica entre as 23 e $5 \mathrm{~h}$, conforme 
me pode ser comparado no item 1.3 o Apêndice $5(\mathrm{ECN})$ com o $6(\mathrm{ECI})$, em que o decréscimo foi, em média, 17,6\%; o Apêndice 7 (EVN) com o 8 (EVI), em que o decréscimo foi, em média, 14,8\%; e o Apêndice 9 (EAN) com o 10 (EAI), em que o decréscimo foi, em média, $9,4 \%$;

v) nos sistemas EAN e EAI o valor foi maior que os demais elétricos devido ao faturamento da demanda também no horário de ponta, cujo valor da tarifa é aproximadamente o triplo em relação ao horário fora de ponta (tanto da tarifa azul como verde, que possui tarifa de demanda única) e 2,65 vezes maior que a tarifa de demanda convencional, embora o faturamento do consumo no horário de ponta desta modalidade seja, em média, 4,66 vezes menor que no horário de ponta da tarifa verde. Isto significa que, se possível, o sistema não deve operar no horário de ponta na modalidade azul e, caso não seja possivel, é melhor então operar o tempo todo (da ponta e do dia), fato que conduz ao redimensionamento do sistema. No item 1.3 dos Apêndices 5, 6, 7, 8, 9 e 10 estão desmembrados os custos de bombeamento do sistema, e no item 1 do Apêndice 2 os preços das tarifas de energia elétrica, que ajudam a esclarecer estes comentários.

Conforme foi discorrido no tópico (v) o redimensionamento do sistema pode ser a melhor opção para redução de custos de bombeamento e total do sistema. Como exemplo, se na presente aplicação o sistema de recalque estivesse suprindo um reservatório para utilização não exclusiva de irrigação, seria melhor que a vazão do sistema, nesta modalidade de tarifação, fosse $258,1 \mathrm{~m}^{3} / \mathrm{h}$ em 19 horas de funcionamento médio diário, do que os $245,19 \mathrm{~m}^{3} / \mathrm{h}$ em 20 horas (volume total bombeado de $4.903,8 \mathrm{~m}^{3} \mathrm{em}$ ambos casos), pois os custos tipo $\mathrm{T} / \mathrm{E}$, estimados pelo modelo, seriam US\$ $59.717,91$ para o CSN; US\$ 6.117,75 para o CFAS; US\$ 781,96 para o CAMR; US\$ 6.823,66 para o CABO e US\$ $13.723,38$ para o CATS, contra os atuais US\$ $60.436,63$ para o CSN; US $\$ 6.191,38$ para o CFAS; US\$ 811,10 para o CAMR; US\$ $12.267,67$ para o CABO e US\$ $19.270,16$ para o CATS. Fato interessante é que mesmo aumentando a vazão do sistema o CSN seria menor que no segundo caso, o que é explicado pela redução do custo da tubulação de recalque, cujo diâmetro econômico teórico passaria de $286,5 \mathrm{~mm}$ para 267,9 $\mathrm{mm}$, sem elevação nos custos de bombeamento, pois economizaria-se o faturamento da demanda no horário de ponta, embora o consumo de energia elétrica tivesse aumentado pela maior exigência de potência da bomba hidráulica (devido a maior altura manométrica e vazão do sistema). Um aspecto desfavorável seria a elevação custo anual de manutenção e reparos "puxado" pela bomba hidráulica e pelo motor elétrico, embora este custo tenha pequena participação no custo anual total do sistema. 
Tabela 11. Valores do custo anual de bombeamento (CABO), em US\$, dos tipos Comercial/Fabricante (C/F), Teórico/Econômico (T/E), Comercial/Teórico/Econômico (C/T/E) e Comercial/Econômico (C/E) nas opções do sistema.

\begin{tabular}{cccccccc}
\hline \hline \multirow{2}{*}{$\begin{array}{c}\text { Tipo de } \\
\text { Custo }\end{array}$} & D & ECN & ECI & EVN & EVI & EAN & EAI \\
\cline { 2 - 8 } & $19.761,89$ & $12.037,16$ & $9.996,31$ & $8.586,26$ & $7.470,51$ & $13.724,14$ & $12.532,05$ \\
Com./Fabr. & $19.960)^{\prime}$ do sistema \\
Teór./Econ. & $16.936,24$ & $10.808,40$ & $9.041,10$ & $7.779,23$ & $6.800,36$ & $12.267,67$ & $11.229,38$ \\
Com/Teor/Econ & $16.966,99$ & $10.680,26$ & $9.279,15$ & $7.967,34$ & $6.932,02$ & $12.168,22$ & $11.111,28$ \\
Com./Econ. & $16.927,71$ & $10.328,16$ & $8.973,23$ & $7.701,89$ & $6.698,13$ & $11.767,07$ & $10.744,97$ \\
\hline \hline
\end{tabular}

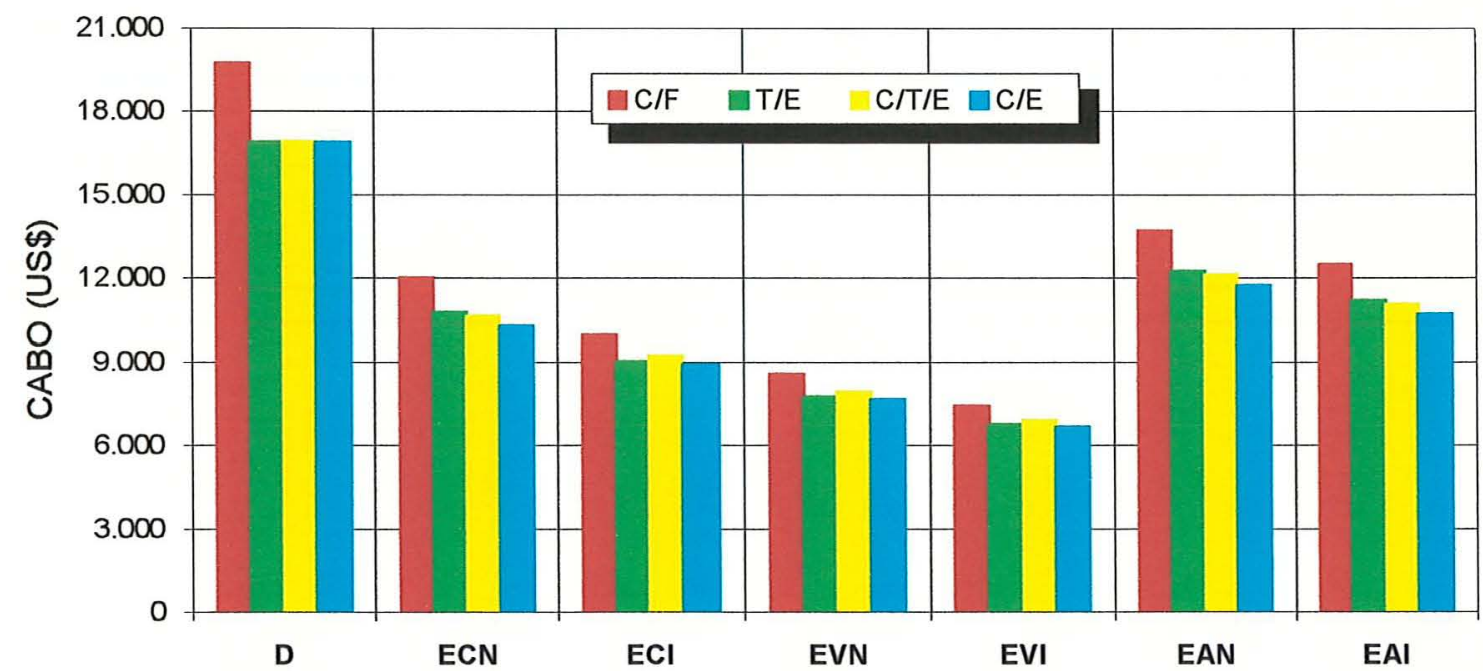

Figura 4. Comportamento do custo anual de bombeamento (CABO) dos tipos Comercial/Fabricante (C/F), Teórico/Econômico (T/E), Comercial/Teórico/Econômico (C/T/E) e Comercial/Econômico (C/E) nas opções do sistema.

Em relação ao custo anual total do sistema (CATS), que é o parâmetro comparativo mais importante para seleção da opção de sistema mais adequado, e para os dados utilizados na aplicação, a Tabela 12 e Figura 5, mostram que:

i) no aspecto geral, seu comportamento é semelhante ao do $\mathrm{CABO}$, pois este é quem tem maior participação na sua composição, ou seja, em termos médios no sistema $\mathrm{D}$ sua participação é de $64 \%$, contra $25 \%$ do CFAS e $11 \%$ do CAMR; no sistema ECN é de $60 \%$, contra $35 \%$ do CFAS e $5 \%$ do CAMR; no sistema ECI é de $57 \%$, contra $38 \%$ do CFAS e $5 \%$ do CAMR; no sistema EVN é de $54 \%$, contra $40 \%$ do CFAS e $6 \%$ do CAMR; no sistema EVI é de $51 \%$, 
contra $43 \%$ do CFAS e $6 \%$ do CAMR; no sistema EAN é de $64 \%$, contra $32 \%$ do CFAS e $4 \%$ do CAMR; e no sistema EAI é de $61 \%$, contra $34 \%$ do CFAS e $5 \%$ do CAMR. Tais proporções explicam, também, a seqüência decrescente dos valores dos diâmetros teóricos econômicos da tubulação de recalque calculados pelo modelo $(300,4 \mathrm{~mm}$ para o sistema $\mathrm{D} ; 386,5 \mathrm{~mm}$ para o sistema EAN; $283,0 \mathrm{~mm}$ no sistema EAI; $281,2 \mathrm{~mm}$ para o sistema ECN; $272,5 \mathrm{~mm}$ para o sistema ECI; $270,2 \mathrm{~mm}$ para o sistema ECN; e $265,5 \mathrm{~mm}$ para o sistema EVI), ou seja, nos sistemas em que o $\mathrm{CABO}$, principalmente, e o CAMR tiveram maior participação o diâmetro foi maior;

ii) no sistema $\mathrm{D}$, a seqüência crescente dos valores para os 4 tipos foram T/E, C/T/E, C/E e C/F, fato que demonstra a coerência do modelo, pois verifica-se, no primeiro caso, o dimensionamento econômico ideal, estimado pelo modelo e que é, portanto, teórico; passando em seguida para o econômico comercial parcial, também estimado pelo modelo; vindo após o econômico comercial real, que na prática é o ideal possível; e, por fim, o comercial conforme o fabricante, que embora também representa valores reais, não são os mais econômicos. Nos sistemas elétricos, a sequência seria a mesma se o rendimento do motor elétrico do sistema $\mathrm{C} / \mathrm{E}$ fosse também $88 \%$ e não os $91 \%$; porém, devido a esta "pequena" diferença, a seqüência crescente foi: $\mathrm{C} / \mathrm{E}, \mathrm{T} / \mathrm{E}, \mathrm{C} / \mathrm{T} / \mathrm{E}$ e C/F;

iii) o tipo T/E superestima, em média, $0,9 \%$ o valor do $\mathrm{C} / \mathrm{E}$, ou seja subestima $1,4 \%$ para o sistema D; e superestima $1,1 \%, 1,3 \%, 1,1 \%, 1,3 \%, 1,4 \%$ e $1,2 \%$ para os sistema $\mathrm{ECN}, \mathrm{ECI}$, EVN, EVI, EAN e EAI, respectivamente. O motivo da precisão das estimativas pelo modelo deve-se, principalmente, ao excelente ajuste da função tipo multiplicativa de custo versus diâmetro ou potência de cada equipamento do sistema;

iv) o sistema à eletricidade na tarifa verde para irrigantes foi o que apresentou os menores valores e, portanto, o mais indicado para o exercício de aplicação, seguido do sistema na tarifa verde normal, convencional irrigante, convencional normal, azul irrigante, azul normal e, por último, o sistema a óleo diesel.

No Apêndice 11 podem ser vistos o comportamento das curvas de custo anual total do sistema, custo anual de bombeamento, custo fixo anual e custo anual com manutenção e reparos para os sistemas D, ECN, ECI, EVN, EVI, EAN e EAI, respectivamente, numa ampla faixa de diâmetros da tubulação de recalque $(\phi)$. 
Tabela 12. Valores do custo anual total do sistema (CATS), em US\$, dos tipos Comercial/Fabricante (C/F), Teórico/Econômico (T/E), Comercial/Teórico/Econômico (C/T/E) e Comercial/Econômico (C/E) nas opções do sistema.

\begin{tabular}{cccccccc}
\hline \hline Tipo de & \multicolumn{8}{c}{ Opção do sistema } \\
\cline { 2 - 8 } Custo & D & ECN & ECI & EVN & EVI & EAN & EAI \\
\hline Com./Fabr. & $29.349,18$ & $19.037,93$ & $16.548,29$ & $15.587,03$ & $14.471,28$ & $20.724,91$ & $19.532,82$ \\
Teór./Econ. & $26.780,29$ & $17.764,86$ & $15.923,47$ & $14.614,93$ & $13.625,71$ & $19.270,16$ & $18.201,64$ \\
Com/Teor/Econ & $26.780,64$ & $17.805,45$ & $15.987,61$ & $14.675,81$ & $13.640,49$ & $19.293,41$ & $18.236,47$ \\
Com./Econ. & $27.158,43$ & $17.572,27$ & $15.720,31$ & $14.448,97$ & $13.445,21$ & $19.011,18$ & $17.989,08$ \\
\hline \hline
\end{tabular}

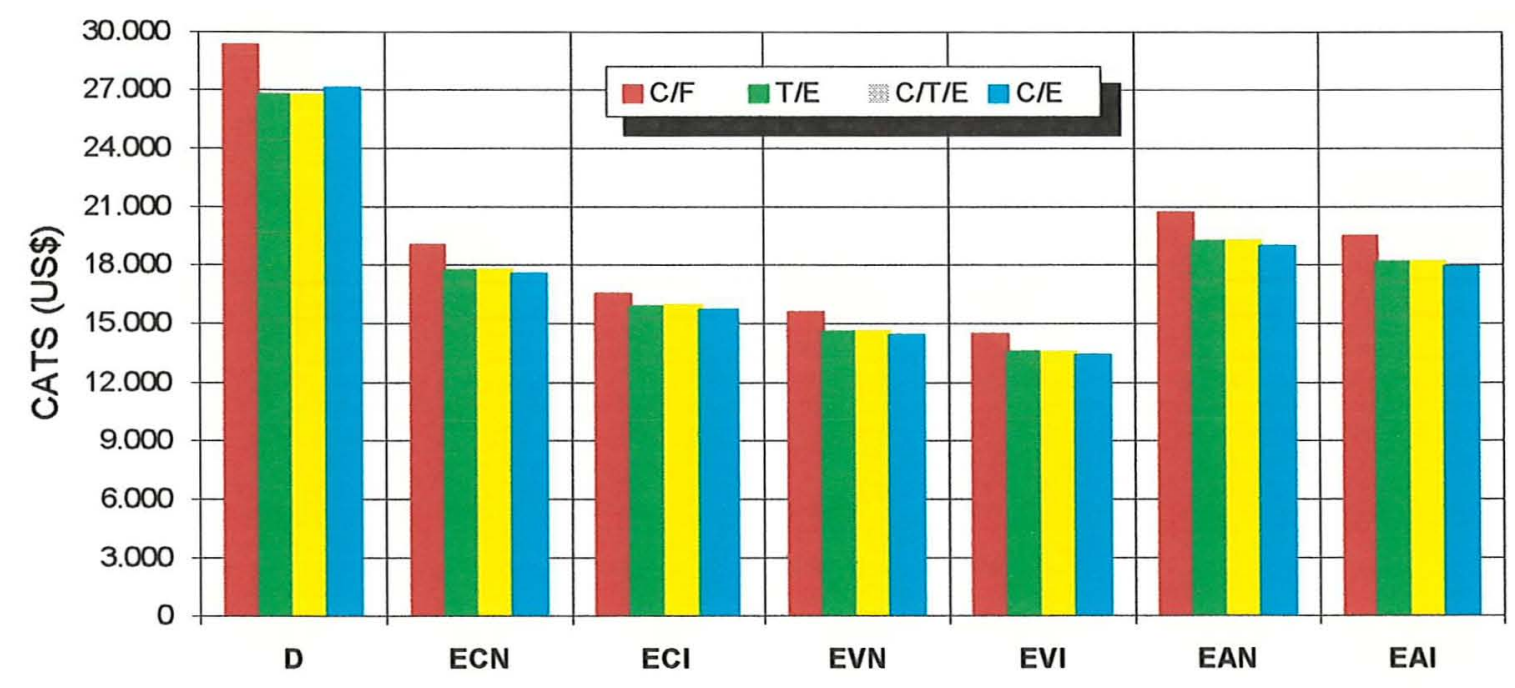

Figura 5. Comportamento do custo anual total do sistema (CATS) dos tipos Comercial/Fabricante (C/F), Teórico/Econômico (T/E), Comercial/Teórico/Econômico (C/T/E) e Comercial/Econômico (C/E) nas opções do sistema.

\subsection{Sensibilidade dos custos do sistema com bomba hidráulica acionada por motor à eletricidade em relação ao comprimento da linha de alta tensão}

Os sistemas com bomba hidráulica acionada por motores elétricos embora apresentem algumas vantagens sobre os com motor diesel, como maior economia operacional, maior vida útil, maior rendimento, partida instantânea nos dias mais frios, além de outras já citadas no item 2 (Revisão de Literatura), muitas vezes apresentam o ponto de bombeamento distante do sistema 
elétrico da concessionária, exigindo uma linha de alta tensão que pode inviabilizar esta forma de energia. Neste item, é verificada a sensibilidade do custo de aquisição do sistema (CSN) e do custo anual total do sistema (CATS), estimados com base no diâmetro econômico teórico (ou custos do tipo T/E do item 4.2), quando se varia o comprimento da linha de alta tensão (LLAT).

Na Tabela 13 são apresentados os custos dos sistemas à eletricidade para alguns comprimentos da linha de alta tensão. Como o custo anual de bombeamento não varia com o LLAT, seu valor foi omitido da tabela, bem como o foram o CFAS e o CAMR, que variam, porém de maneira semelhante ao CSN. Os custos do sistema a óleo diesel foram apresentados somente para comparação com os à eletricidade.

Tabela 13. Variação do custo do sistema novo (CSN), em US\$, e custo anual total do sistema (CATS), em US\$, para diferentes comprimentos da linha de alta tensão (LLAT).

\begin{tabular}{ccccc}
\hline $\begin{array}{c}\text { Opcão do } \\
\text { Sistema }\end{array}$ & $\begin{array}{c}\text { LLAT } \\
(\mathbf{m})\end{array}$ & $\begin{array}{c}\boldsymbol{\phi}_{\mathbf{e}} \\
(\mathbf{m m})\end{array}$ & $\begin{array}{c}\text { CSN } \\
\text { (USS) }\end{array}$ & $\begin{array}{c}\text { CATS } \\
\text { (USS) }\end{array}$ \\
\hline Diesel & - & 300,4 & $66.464,57$ & $29.349,18$ \\
\hline ECN & 1.800 & 281,2 & $60.004,41$ & 17.764 .86 \\
& 3.600 & & $73.684,41$ & $19.248,37$ \\
& 5.400 & & $87,364,41$ & $20.731,89$ \\
& 7.200 & & $101.044,41$ & $22.215,41$ \\
\hline ECI & 1.800 & 272,5 & $59.306,47$ & $15.923,47$ \\
& 3.600 & & $72.986,47$ & $17.406,99$ \\
& 5.400 & & $86.666,47$ & $18.890,50$ \\
& 7.200 & & $100.346,47$ & $20.374,02$ \\
\hline EVN & 1.800 & 270,2 & $59.129,96$ & $14.642,93$ \\
& 3.600 & & $72.809,96$ & $16.126,44$ \\
& 5.400 & & $86.489,96$ & $17.609,96$ \\
& 7.200 & & $100.169,96$ & $19.093,48$ \\
\hline EVI & 1.800 & 265,5 & $58.766,73$ & $13.625,71$ \\
& 3.600 & & $72.446,73$ & $15.109,23$ \\
& 5.400 & & $86.126,73$ & $16.592,75$ \\
& 7.200 & & $99.806,73$ & $18.076,26$ \\
\hline EAN & 1.800 & 286,5 & $60.436,63$ & 19.270 .16 \\
& 3.600 & & $74.116,63$ & $20.753,67$ \\
& 5.400 & & $87.796,63$ & $22.237,19$ \\
& 7.200 & & $101.476,63$ & $23.720,71$ \\
\hline EAI & 1.800 & 283,0 & $60.152,93$ & $18.201,64$ \\
& 3.600 & & $73.832,93$ & $19.685,15$ \\
& 5.400 & & $87.512,93$ & $21.168,67$ \\
& 7.200 & & $101.192,93$ & $22.652,19$ \\
\hline \hline
\end{tabular}


Com base na Tabela 13, foram calculados, para cada sistema, os coeficientes das funções lineares existentes entre os custos e o comprimento da linha de alta tensão, ou seja: CSN $=\mathrm{A}_{1}+\mathrm{B}_{1}$.LLAT e CATS $=\mathrm{A}_{2}+\mathrm{B}_{2}$.LLAT. Com isso, foram obtidos os comprimentos das linhas de alta tensão, $\operatorname{LLAT}_{1(\mathrm{e}=\mathrm{d})}$ e $\operatorname{LLAT}_{2(\mathrm{e}=\mathrm{d})}$, que igualam, respectivamente, os custos de aquisição (CSN) e anual total (CATS) de um sistema elétrico com o diesel, ou seja, no primeiro caso indicase apenas o comprimento da linha de alta tensão que proporciona igual custo de aquisição entre ambos sistemas, não se considerando o custo operacional. Já no segundo caso, considera-se também os custos operacionais do sistema (CABO e CAMR), que transcorrerão durante a vida útil (ou período de depreciação) do sistema, sendo portanto mais importante para tomada de decisão. A Tabela 14 apresenta os valores destes parâmetros, bem como os coeficientes das funções lineares.

Tabela 14. Valores dos coeficientes $A_{1} / B_{1}$ e $A_{2} / B_{2}$ das funções lineares de custo do sistema novo (CSN) e custo anual total (CATS), respectivamente, em relação do comprimento da linha de alta tensão (LLAT); valores dos comprimentos da linha de alta tensão que igualam os custos do sistema diesel com o elétrico, $\operatorname{LLAT}_{1(\mathrm{e}=\mathrm{d})}$ e $\operatorname{LLAT}_{2(\mathrm{e}-\mathrm{d})}$; e custo do sistema novo para o comprimento da linha de alta tensão que iguala o custo anual total do sistema elétrico com o diesel, $\mathrm{CSN}_{2(\mathrm{e}=\mathrm{d})}$.

\begin{tabular}{|c|c|c|c|c|c|c|}
\hline & \multicolumn{6}{|c|}{ Opção do sistema } \\
\hline & ECN & ECI & EVN & EVI & $\mathbf{E A N}$ & EAI \\
\hline $\mathbf{A}_{1}$ (USS) & $46.324,41$ & $45.622,47$ & $45.449,96$ & $45.086,73$ & $46.756,63$ & $46.472,93$ \\
\hline$B_{1}(U S \$ / m)$ & 7,60 & 7,60 & 7,60 & 7,60 & 7,60 & 7,60 \\
\hline LLAT $_{1(\mathrm{e}=\mathrm{d})}(\mathrm{m})$ & $2.650,0$ & $2.741,9$ & $2.765,1$ & $2.812,9$ & $2.593,15$ & $2.630,5$ \\
\hline $\mathbf{A}_{2}$ (US\$) & $16.281,34$ & $14.439,79$ & $13.159,24$ & $12.142,02$ & $17.786,64$ & $16.717,95$ \\
\hline $\mathbf{B}_{2}(\mathbf{U S} \$ / \mathbf{m})$ & 0,8242 & 0,8242 & 0,8242 & 0,8242 & 0,8242 & 0,8242 \\
\hline $\mathbf{L L A T}_{2(\mathrm{e}=\mathrm{d})}(\mathrm{m})$ & $12.738,2$ & $14.972,7$ & $16.526,4$ & $17.760,6$ & $10.912,0$ & $12.208,6$ \\
\hline $\mathrm{CSN}_{2(\mathrm{e}=\mathrm{d})}$ (USS) & $143.136,25$ & $159.414,99$ & $171.050,60$ & $180.067,29$ & $129.687,83$ & $139.258,29$ \\
\hline
\end{tabular}

Conforme a Tabela 14, verifica-se que o coeficiente $B_{1}$, corresponde ao próprio custo unitário de aquisição da linha de alta tensão (US\$ 7,60/m). Também é verificado, com base nos valores médios e para os dados utilizados neste exemplo de aplicação, que se o comprimento da linha de alta tensão fosse aproximadamente 2.700 metros, o investimento inicial que o proprietário faria seria o mesmo para adquirir um sistema diesel ou elétrico. Porém, devido aos maiores 
etário faria seria o mesmo para adquirir um sistema diesel ou elétrico. Porém, devido aos maiores custos operacionais durante os 10 anos considerados para a depreciação, o sistema elétrico seria mais vantajoso até o limite de 14.186 metros, que corresponde ao comprimento para equiparação dos custos totais, embora neste caso o investimento inicial seria aproximadamente $131 \%$ superior no sistema elétrico em relação ao diesel. Obviamente, estas considerações são válidas somente neste caso específico, ou seja, se um ou mais parâmetros forem alterados, como por exemplo o número de horas de funcionamento do sistema no ano ou o periodo de depreciação, novos valores serão obtidos na Tabela 14. Outra consideração relevante é que além do aspecto econômico existem outros pouco quantificáveis que não foram analisados, como por exemplo, a maior independência de funcionamento do motor diesel em relação ao elétrico, que normalmente está interligado ao sistema elétrico da concessionária e, portanto, sujeito a seus regulamentos. 


\section{CONCLUSÕES}

Conforme a metodologia proposta e para as condições consideradas na aplicação pode-se concluir que:

i) O modelo matemático superestimou, em média, 0,9\% o custo anual total do sistema na condição econômica comercial, ou seja, subestimou $1,4 \%$ para o sistema a diesel; e superestimou $1,1 \%, 1,3 \%, 1,1 \%, 1,3 \%, 1,4 \%$ e $1,2 \%$ para os sistemas elétricos na: tarifa convencional normal, tarifa convencial com desconto para irrigantes, tarifa verde normal, tarifa verde com desconto para irrigantes, tarifa azul normal e tarifa azul com desconto para irrigante, respectivamente. A precisão destas estimativas ocorreu, principalmente, pelo excelente ajuste da função tipo multiplicativa de custo versus diâmetro ou potência de cada equipamento do sistema;

ii) O custo de aquisição e os custos anuais fixo; de bombeamento; de manutenção e reparos; e total do sistema a diesel foram superiores ao elétrico em todas as modalidades de tarifação da energia;

iii) Entre as modalidades de tarifação da energia elétrica, a horo-sazonal verde foi a que se apresentou mais vantajosa, seguida da convencional e da horo-sazonal azul. Também verificou-se, caso o sistema de recalque fosse utilizado exclusivamente para irrigação, que o proprietário deveria optar pelo desconto especial sobre a tarifa de consumo de energia elétrica durante o período das 23 as 5 horas, pois propiciaria, em média, $17,6 \%, 14,8 \%$ e $9,4 \%$ de redução no custo de bombeamento em relação à tarifa normal para as modalidades convencional, horo-sazonal verde e horo-sazonal azul;

iv) A análise de sensibilidade dos custos em relação ao comprimento da linha de alta tensão mostrou que, em média, com o comprimento de 2.700 metros, o custo de aquisição seria o mesmo entre o sistema a diesel e à eletricidade. Porém, devido ao maior custo operacional, o sistema à eletricidade seria mais vantajoso até o limite de 14.186 metros, que corresponde à equiparação dos custos totais, embora neste caso o seu custo de aquisição seria $131 \%$ superior. 


\section{REFERÊNCIAS BIBLIOGRÁFICAS}

ALVES, C.B. Parâmetros energéticos na elaboração de programa de irrigação para o distrito de Santa Terezinha - MS. Botucatu, 1990. 122p. Tese (Doutorado) - Faculdade de Ciências Agronômicas, Universidade Estadual Paulista “Júlio de Mesquita Filho".

AZEVEDO NETO, J.M. Manual de hidráulica. 7.ed. São Paulo: Edgard Blücher, 1991. 2v.

BABBITT, H.E. Abastecimento de água. Trad. de Z.C.Branco. São Paulo: Edgard Blücher; Brasília: INL, 1973, 592p.

BHAVE, P.B. Optimal expansion of water distribution systems. Journal of the environmental engineering division - ASCE, v.111, n.2, p.177-197, Apr. 1985.

BRASIL. Ministério da Infra-estrutura. Secretaria Nacional de Energia. Departamento Nacional de Águas e Energia Elétrica. Portaria nº 105, de 3 de abril de 1992. Diário Oficial, 06 fev. 1993.

BRASIL. Ministério da Irrigação. Programa Nacional de Irrigação - PRONI. Tempo de irrigar: manual do irrigante. São Paulo: Mater, 1987. 160p.

CAMP, T.R. Water distribution. In: DAVIS, C.V. Handbook of applied hydraulics. New York: McGraw-Hill, 1952. cap.20, p.881-944.

CARVALHO, D.F. Instalações elevatórias - bombas. 4.ed. Belo Horizonte: IPUC, Departamento de Engenharia Civil, 1989. 355p.

CHIPLUNKAR, A.V.; MEHNDIRATTA, S.L.; KHANNA, P. Looped water distribution system optimization for single loading. Journal of the environmental engineering division ASCE, v.112, n.2, p.264-279, Apr. 1986.

COELHO, S.T. Matemática financeira e análise de investimentos. São Paulo: EDUSP, 1979. $279 \mathrm{p}$. 
COIADO, E.M.; RIVELLI JUNIOR., A. Influência da evolução do custo da energia elétrica no diâmetro econômico de uma instalação de recalque de água de abastecimento. Revista Brasileira de Engenharia - Caderno de Recursos Hídricos, v.11, n.2, p.27-48, dez. 1993.

COMITÊ DE DISTRIBUIÇÃO DE ENERGIA ELÉTRICA. Tarifas horo-sazonais: manual de orientação ao consumidor. Rio de Janeiro, 1988. 28p.

CUOMO, A.R.; VILLELA, S.M. Dimensionamento econômico de tubulações em recalque. São Carlos: EESC, 1961. (Publicação 46).

DAKER, A. Hidráulica aplicada à agricultura: a água na agricultura. 7.ed. Rio de Janeiro: Freitas Bastos, 1987. 3v.

DEB, A.K. Optimization in design of pumping systems. Journal of the environmental engineering division - ASCE, v.104, n.EE1, p.127-136, Feb. 1978.

FOLHA DE SÃo PAUlO. Novo hábito pode reduzir $15 \%$ do consumo. Folha de São Paulo, São Paulo, 29 abr. 1997, Cotidiano, p.8.

FOLHA DE SÃo PAULO. Tarifa maior deve ser arma contra blecaute. Folha de São Paulo, São Paulo, 29 abr. 1997, Cotidiano, p.6.

FRANKE, A.E.; DORFMAN, R. Análise comparativa dos custos de acionamento dos conjuntos motobomba através da energia elétrica, fóssil ou biomassa. (compact disc) In: CONGRESSO BRASILEIRO DE ENGENHARIA RURAL, 26. Campina Grande: SBEA, 1997.

FRIZZONE, J.A.; BOTREL, T.A.; FREITAS, H.A.C. Análise comparativa dos custos de irrigação por pivô central em cultura de feijão utilizando energia elétrica e óleo diesel. Engenharia Rural, v.5, n. 1, p.34-53, julho 1994.

GARCEZ, L.N. Da condição de mínimo custo nos condutos forçados. Aplicação ao cálculo das redes de distribuição de água potável. São Paulo, 1946. 99p. Tese (Catedrático) - Escola Politécnica, Universidade de São Paulo.

GONÇALVES, M.; OKAMOTO, T.; IGAWA, K.A. Uma experiência da Eletropaulo com irrigação. In: CONFERÊNCIA LATINO AMERICANA DE ELETRIFICAÇÃO RURAL, 11., Curitiba, 1986. Anais. Curitiba: 1986. p.815-30.

HOFFMANN, R.; ENGLER, J.J.C.; SERRANO, O.; THAME, A.C.M.; NEVES, E.M. Administração da empresa agrícola. 5 ed. São Paulo: Pioneira, 1987. 325p. 
LENCASTRE, A. Manual de hidráulica geral. São Paulo: Edgard Blücher, 1972. 411p.

LENCASTRE, A. Hidráulica geral. Lisboa, 1983.654p.

MARTINS, J.A. Limites de velocidade e pré-dimensionamento de condutos principais das redes de distribuição de água potável. Condições de mínimo custo. São Paulo, 1966. 188p. Tese (Catedrático) - Escola Politécnica, Universidade de São Paulo.

MATAIX, C. Mecanica de fluidos y maquinas hidraulicas. Madrid: Castillo; New York: Harper \& Row, 1970. 582p.

MELO, J.F. Custos da irrigação por aspersão em Minas Gerais. Viçosa, 1993. 147p. Dissertação (M.S.) - Universidade Federal de Viçosa.

NEVES, E.T. Curso de hidráulica. 7.ed. Porto Alegre: Globo, 1982. 577p.

PIEDADE JÚNIOR, C. Eletrificação rural. 2.ed. São Paulo: Nobel, 1983. 280p.

SENTELHAS, P.C.; FIGUEIREDO, S.F.; COELHO, R.D. Viabilidade econômica do uso da tarifação noturna de energia elétrica para irrigação em regiões com diferentes demandas evapotranspirativas. (compact disc). In: CONGRESSO BRASILEIRO DE ENGENHARIA RURAL, 26. Campina Grande: SBEA, 1997.

SCALOPPI, E.J. Exigências de energia para irrigação. ITEM - Irrigação e Tecnologia Moderna, Brasília, n.21, p.13-7, Jun. 1985.

TSUTIYA, M.T. Redução do custo de energia elétrica em estações elevatórias de sistemas de abastecimento de água de pequenos e médio portes. São Paulo, 1989. 207p. Tese (Doutorado) - Escola Politécnica, Universidade de São Paulo.

ZOCOLER, J.L. Custos da irrigação por aspersão convencional em função da pressão de operação, diâmetros dos bocais e espaçamentos dos aspersores. Piracicaba, 1994. 120p. Dissertação (Mestrado) - Escola Superior de Agricultura "Luiz de Queiroz", Universidade de São Paulo. 


\section{APÊNDICE 1. Custos dos equipamentos hidráulicos e elétricos novos em função dos diâmetros e potências comerciais, respectivamente.}

\section{Equipamentos hidráulicos}

1.1. Tubo de aço galvanizado flangeado de 6 metros de comprimento (KREBSFER):

\begin{tabular}{|c|c|c|c|c|c|c|c|c|c|c|c|}
\hline $\begin{array}{c}\text { Diâmetro } \\
\text { (mm) }\end{array}$ & 75 & 100 & 125 & 150 & 175 & 200 & 250 & 300 & 350 & 400 & 500 \\
\hline $\begin{array}{c}\text { Custo } \\
\text { (USS/tubo) }\end{array}$ & 30,00 & 38,00 & 62,00 & 82,00 & 109,00 & 125,00 & 152,00 & 192,00 & 226,00 & 286,00 & 415,00 \\
\hline
\end{tabular}

1.2. Curva de $60^{\circ}$ de aço galvanizado flangeada (KREBSFER):

\begin{tabular}{|c|c|c|c|c|c|c|c|c|}
\hline $\begin{array}{c}\text { Diâmetro } \\
\text { (mm) }\end{array}$ & 75 & 100 & 125 & 150 & 175 & 200 & 250 & 300 \\
\hline $\begin{array}{c}\text { Custo } \\
\text { (USS/peça) }\end{array}$ & 23,00 & 25,00 & 26,00 & 29,00 & 34,00 & 44,00 & 59,00 & 84,00 \\
\hline
\end{tabular}

1.3. Curva de $90^{\circ}$ de aço galvanizado flangeada (composta de 1 curva $30^{\circ}$ e 1 curva de $60^{\circ}$ ):

\begin{tabular}{|c|c|c|c|c|c|c|c|c|}
\hline $\begin{array}{c}\text { Diâmetro } \\
\text { (mm) }\end{array}$ & 75 & 100 & 125 & 150 & 175 & 200 & 250 & 300 \\
\hline $\begin{array}{c}\text { Custo } \\
\text { (USS/peça) }\end{array}$ & 45,00 & 51,00 & 50,00 & 55,00 & 64,00 & 84,00 & 109,00 & 154,00 \\
\hline
\end{tabular}

1.4. Válvula de sucção de aço galvanizado flangeada (KREBSFER):

\begin{tabular}{|c|c|c|c|c|c|c|c|c|}
\hline $\begin{array}{c}\text { Diâmetro } \\
\text { (mm) }\end{array}$ & 75 & 100 & 125 & 150 & 175 & 200 & 250 & 300 \\
\hline $\begin{array}{c}\text { Custo } \\
\text { (USS/peça) }\end{array}$ & 45,00 & 46,00 & 65,00 & 76,00 & 96,00 & 120,00 & 179,00 & 273,00 \\
\hline
\end{tabular}

1.5. Válvula de retenção flangeada com registro de retorno (KREBSFER):

\begin{tabular}{|c|c|c|c|c|c|c|c|c|}
\hline $\begin{array}{c}\text { Diâmetro } \\
\text { (mm) }\end{array}$ & 75 & 100 & 125 & 150 & 175 & 200 & 250 & 300 \\
\hline $\begin{array}{c}\text { Custo } \\
\text { (USS/peça) }\end{array}$ & 180,00 & 200,00 & 220,00 & 250,00 & 280,00 & 320,00 & 350,00 & 420,00 \\
\hline
\end{tabular}

1.6. Válvula de gaveta de ferro fundido flangeada, PN-10, V. Bronze (BRUZANTIN):

\begin{tabular}{|c|c|c|c|c|c|c|c|c|c|c|}
\hline $\begin{array}{c}\text { Diâmetro } \\
\text { (mm) }\end{array}$ & 50 & 75 & 100 & 125 & 150 & 200 & 250 & 300 & 350 & 400 \\
\hline $\begin{array}{c}\text { Custo } \\
\text { (USS/peça) }\end{array}$ & 205,12 & 284,07 & 297,44 & 416,28 & 500,76 & 769,22 & $1.170,44$ & $1.862,74$ & $2.485,06$ & $3.070,88$ \\
\hline
\end{tabular}


1.7. Luva cônica concêntrica (ampliação) de aço galvanizado flangeada (KREBSFER):

\begin{tabular}{|c|c|c|c|c|c|c|c|c|c|c|}
\hline $\begin{array}{c}\text { Dimensão } \\
\text { (pol xpol) }\end{array}$ & $4 \times 3$ & $5 \times 4$ & $6 \times 4$ & $6 \times 5$ & $7 \times 5$ & $7 \times 6$ & $8 \times 6$ & $8 \times 7$ & $10 \times 8$ & $12 \times 10$ \\
\hline $\begin{array}{c}\text { Diâmetro(1) } \\
\text { (mm) }\end{array}$ & 100 & 125 & 150 & 150 & 175 & 175 & 200 & 200 & 250 & 300 \\
\hline $\begin{array}{c}\text { Custo } \\
\text { (USS/peca) }\end{array}$ & 13,00 & 16,00 & 25,00 & 26,00 & 33,00 & 34,00 & 37,00 & 38,00 & 44,00 & 60,00 \\
\hline
\end{tabular}

(1) Para a regressão multiplicativa, considerou-se o diâmetro maior do equipamento (m).

1.8. Luva cônica excêntrica (redução) de aço galvanizado flangeada (KREBSFER):

\begin{tabular}{|c|c|c|c|c|c|c|c|c|c|c|c|c|}
\hline $\begin{array}{c}\text { Dimensão } \\
\text { (pol xpol) }\end{array}$ & $5 \times 3$ & $5 \times 4$ & $6 \times 3$ & $6 \times 5$ & $7 \times 4$ & $7 \times 5$ & $8 \times 5$ & $8 \times 6$ & $10 \times 6$ & $10 \times 8$ & $12 \times 6$ & $12 \times 8$ \\
\hline $\begin{array}{c}\text { Diâmetro (1) } \\
\text { (mm) }\end{array}$ & 100 & 125 & 150 & 150 & 175 & 175 & 200 & 200 & 250 & 250 & 300 & 300 \\
\hline $\begin{array}{c}\text { Custo } \\
\text { (USS/peça) }\end{array}$ & 39,00 & 40,00 & 42,00 & 48,00 & 54,00 & 56,00 & 62,00 & 72,00 & 84,00 & 105,00 & 106,00 & 116,00 \\
\hline
\end{tabular}

(1) Para a regressão multiplicativa, considerou-se o diâmetro maior do equipamento (m).

1.9. Bomba hidráulica centrífuga multiestágios - WKL - 1750 rpm (KSB):

\begin{tabular}{|c|c|c|c|c|c|c|}
\hline Modelo $^{(1)}$ & $80 / 3$ & $80 / 5$ & $80 / 7$ & $100 / 3$ & $100 / 5$ & $100 / 7$ \\
\hline $\begin{array}{c}\text { Potência }^{(2)} \\
(W)\end{array}$ & 12.000 & 20.000 & 28.000 & 37.000 & 62.000 & 86.500 \\
\hline $\begin{array}{c}\text { Custo } \\
\text { USS/peça) }^{(\text {) }}\end{array}$ & $5.446,58$ & $7.156,98$ & $7.993,99$ & $7.114,54$ & $9.031,15$ & $10.935,63$ \\
\hline Modelo $^{(1)}$ & $125 / 3$ & $125 / 4$ & $125 / 5$ & $150 / 2$ & $150 / 3$ & $150 / 4$ \\
\hline $\begin{array}{c}\text { Potência }^{(2)} \\
\text { (W) }\end{array}$ & 93.500 & 125.000 & 156.000 & 127.000 & 191.000 & 254.000 \\
\hline $\begin{array}{c}\text { Custo } \\
\text { (USS/peça) }\end{array}$ & $11.736,26$ & $13.324,88$ & $15.291,41$ & $15.942,27$ & $18.328,70$ & $20.708,14$ \\
\hline
\end{tabular}

(1) No modelo, o primeiro número indica o diâmetro do bocal do recalque $(\mathrm{mm})$ e o segundo 0 número de estágios da bomba;

(2) A potência utilizada na regressão multiplicativa foi obtida através da curva característica do modelo para cada estágio, sendo considerado o valor (da potência) no máximo rendimento;

\section{Equipamentos elétricos}

2.1. Transformador trifásico classe $15 \mathrm{kV}$ (ZAGO):

\begin{tabular}{|c|c|c|c|c|c|c|c|c|}
\hline $\begin{array}{c}\text { Potência } \\
\text { (VA) }\end{array}$ & 15.000 & 30.000 & 45.000 & 75.000 & 112.500 & 150.000 & 225.000 & 300.000 \\
\hline $\begin{array}{c}\text { Custo } \\
\text { (USS/peça) }\end{array}$ & 864,00 & $1.122,00$ & $1.415,00$ & $2.090,00$ & $2.800,00$ & $3.236,00$ & $4.938,00$ & $5.785,00$ \\
\hline
\end{tabular}


2.2. Chave (comando) de partida compensadora em caixa metálica, $380 \mathrm{~V}$ - ligação triângulo (WEG):

\begin{tabular}{|c|c|c|c|c|c|c|c|c|c|c|}
\hline $\begin{array}{c}\text { Potência } \\
\text { (W) }\end{array}$ & 11.000 & 18.500 & 30.000 & 45.000 & 75.000 & 90.000 & 110.000 & 150.000 & 185.000 & 220.000 \\
\hline $\begin{array}{c}\text { Custo } \\
\text { (USS/peça) }\end{array}$ & $1.948,08$ & $2.520,54$ & $2.697,25$ & $3.495,35$ & $4.946,30$ & $6.294,46$ & $6.676,08$ & $10.170,21$ & $11.630,65$ & $14.896,45$ \\
\hline
\end{tabular}

2.3. Banco de capacitores trifásico, $380 \mathrm{~V}$, imerso em óleo (WEG):

\begin{tabular}{|c|c|c|c|c|c|c|c|c|c|c|}
\hline $\begin{array}{c}\text { Potência } \\
\text { (VAr) }\end{array}$ & 12.500 & 15.000 & 20.000 & 22.500 & 25.000 & 30.000 & 35.000 & 40.000 & 45.000 & 50.000 \\
\hline $\begin{array}{c}\text { Custo } \\
\text { (USS/peça) }\end{array}$ & 238,79 & 246,83 & 273,62 & 314,46 & 346,65 & 372,22 & 452,62 & 478,19 & 551,21 & 576,78 \\
\hline
\end{tabular}

2.4. Motor trifásico IP - 54, 1800 rpm (4 pólos), 220/380 ou $380 / 660 \mathrm{~V}$, fechado, ventilador externo, isolamento classe $\mathrm{B}\left(130^{\circ} \mathrm{C}\right), 60 \mathrm{~Hz}$ (WEG):

\begin{tabular}{|c|c|c|c|c|c|c|c|c|c|c|}
\hline $\begin{array}{c}\text { Potência } \\
\text { (W) }\end{array}$ & 3.700 & 7.500 & 15.000 & 22.000 & 37.000 & 55.000 & 75.000 & 110.000 & 150.000 & 185.000 \\
\hline $\begin{array}{c}\text { Custo } \\
\text { (USS/peça) }\end{array}$ & 220,43 & 379,99 & 653,54 & $1.087,75$ & $1.612,07$ & $2.634,21$ & $3.091,97$ & $4.993,59$ & $6.390,46$ & $7.545,11$ \\
\hline
\end{tabular}

\section{Motor diesel}

Motor diesel completo para bombeamento:

\begin{tabular}{|c|c|c|c|c|c|c|}
\hline $\begin{array}{c}\text { Marca } \\
\text { Modelo } \\
\text { Potência (cv) }\end{array}$ & $\begin{array}{c}\text { MWM } \\
\text { D 229-3 } \\
42\end{array}$ & $\begin{array}{c}\text { MWM } \\
\text { D } 229-4 \\
56\end{array}$ & $\begin{array}{c}\text { MWM } \\
\text { D 229-6 } \\
85\end{array}$ & $\begin{array}{c}\text { MWM } \\
\text { TD 229-6 } \\
112\end{array}$ & $\begin{array}{c}\text { MWM } \\
610 \mathrm{~T} \\
164\end{array}$ & $\begin{array}{c}\text { MWM } \\
610 \mathrm{TCA} \\
195\end{array}$ \\
\hline $\begin{array}{c}\text { Potência } \\
(W)\end{array}$ & 30.870 & 41.160 & 62.475 & 82.320 & 120.540 & 143.325 \\
\hline $\begin{array}{c}\text { Custo } \\
\text { (USS/peca) }\end{array}$ & $22.480,34$ & $23.783,00$ & $28.340,96$ & $32.516,70$ & $36.125,62$ & $38.333,08$ \\
\hline $\begin{array}{c}\text { Marca } \\
\text { Modelo }\end{array}$ & $\begin{array}{c}\text { SCÂNIA } \\
\text { DS } 11 \\
300\end{array}$ & $\begin{array}{c}\text { SCÂNIA } \\
\text { DSI } 11\end{array}$ & $\begin{array}{c}\text { SCÂNIA } \\
\text { DSC } 11\end{array}$ & $\begin{array}{c}\text { SCÂNIA } \\
\text { DSI } 14 \\
420\end{array}$ & $\begin{array}{c}\text { SCÂNIA } \\
\text { DSC } 14 \\
440\end{array}$ & \\
\hline Potência (cv) & 300 & 321 & 321 & 420 & 440 & \\
\hline $\begin{array}{l}\text { Potência } \\
\text { (W) }\end{array}$ & 220.500 & 235.935 & 235.935 & 308.700 & 323.400 & \\
\hline $\begin{array}{l}\text { Custo } \\
\text { (USS/peça) }\end{array}$ & $60.736,26$ & $68.039,10$ & $69.271,52$ & $85.919,90$ & $86.512,72$ & \\
\hline
\end{tabular}


APÊNDICE 2. Preços das tarifas de energia elétrica (1); descontos especiais por região para os irrigantes exclusivos (2); e preço do óleo diesel nos postos de combustiveis (3).

1. Preços das tarifas de energia elétrica aplicadas aos consumidores do Subgrupo A4 e B2 (consumidores rurais) nas modalidades horo-sazonais e convencional:

Concessionária: CESP

Dólar comercial para conversão: $\mathrm{R} \$ 1,02$

\begin{tabular}{|c|c|c|c|c|c|}
\hline MODALIDADE & PERIOODO & HORÁRIO & $\begin{array}{c}\text { CONSUMO } \\
\text { US\$ } / \mathrm{kWh}\end{array}$ & $\begin{array}{c}\text { DEMANDA } \\
\text { US\$ } / \mathrm{kW}\end{array}$ & $\begin{array}{l}\text { ULTRAPASSAGEM } \\
\text { DE DEMANDA } \\
\text { US } \$ / \mathrm{kW}\end{array}$ \\
\hline \multirow{4}{*}{ VERDE } & \multirow[t]{2}{*}{ Úmido } & Ponta & 0,30532 & 3,49 & 11,63 \\
\hline & & Fora de Ponta & 0,02883 & 3,49 & 11,63 \\
\hline & \multirow[t]{2}{*}{ Seco } & Ponta & 0,31041 & 3,49 & 11,63 \\
\hline & & Fora de Ponta & 0,03261 & 3,49 & 11,63 \\
\hline \multirow{4}{*}{ AZUL } & \multirow[t]{2}{*}{ Úmido } & Ponta & 0,06349 & 10,46 & 34,87 \\
\hline & & Fora de Ponta & 0,02883 & 3,49 & 11,63 \\
\hline & \multirow[t]{2}{*}{ Seco } & Ponta & 0,06860 & 10,46 & 34,87 \\
\hline & & Fora de Ponta & 0,03261 & 3,49 & 11,63 \\
\hline $\begin{array}{c}\text { CONVENCIONAL } \\
\text { A4 }\end{array}$ & $\begin{array}{c}\text { Úmido } \\
\text { e } \\
\text { Seco } \\
\end{array}$ & $\begin{array}{c}\text { Ponta } \\
\mathrm{e} \\
\text { Fora de Ponta } \\
\end{array}$ & 0,05794 & 3,95 & Isento \\
\hline $\begin{array}{l}\text { CONVENCIONAL } \\
\text { B2 }\end{array}$ & $\begin{array}{c}\text { Úmido } \\
\mathrm{e} \\
\text { Seco }\end{array}$ & $\begin{array}{c}\text { Ponta } \\
\mathrm{e} \\
\text { Fora de Ponta }\end{array}$ & 0,07324 & Isento & Isento \\
\hline
\end{tabular}

OBS: Nos valores das tarifas aplicadas aos consumidores do Subgrupo A4, já está incluido o desconto de $10 \%$ que lhes são concedidos.

2. Descontos especiais para consumidores rurais irrigantes exclusivos, conforme a Portaria 105 do DNAEE:

\begin{tabular}{|l|c|c|}
\hline \multicolumn{1}{|c|}{ REGIĨO DO PAÍS } & \multicolumn{2}{|c|}{ DESCONTO (\%) } \\
\hline & $\begin{array}{c}\text { SUBGRUPO } \\
\text { A4 }\end{array}$ & $\begin{array}{c}\text { SUBGRUPO } \\
\text { B2 }\end{array}$ \\
\hline $\begin{array}{l}\text { Nordeste e regiōes geoeconômicas denominadas Vale do Jequiti- } \\
\text { nhonha e Poligono da Seca, no Estado de Minas Gerais }\end{array}$ & 90 & 73 \\
\hline Norte e Centro-Oeste e demais regiões de Minas Gerais & 80 & 67 \\
\hline Demais Regiōes do Brasil & 70 & 60 \\
\hline
\end{tabular}

3. Preço médio do óleo diesel nos postos de combustíveis (correspondente ao parâmetro $\mathrm{C}_{\mathrm{u}} \mathrm{C}$ - custo da unidade de combustivel): $378,64 \mathrm{US} \$ / \mathrm{m}^{3}$ ( $\cong 0,386 \mathrm{R} \$ /$ litro). 


\section{APÊNDICE 3. Dados técnicos do sistema de irrigação tipo pivô central (fabricante).}

\section{Precipitação diária:}

Lâmina líquida. $7,65 \mathrm{~mm} / \mathrm{dia}$

Eficiência da aplicação.... $85 \%$

Lâmina bruta $9,00 \mathrm{~mm} / \mathrm{dia}$ (24 horas/dia)

2. Descrição e dados técnicos do pivô:

Modelo 4871-PA-VSN/9-1.080

Composição 5 Lances longos $6,5 / 8 "$

4 Lances médios $6,5 / 8 "$

Balanço de $18 \mathrm{~m}$

Sem canhão final

Número de aspersores 213 com tubo de descida

Altura do pivô. $2,7 \mathrm{~m}$ (baixo)

Área circular irrigada 65,38 ha

Lâmina bruta por giro (relê a $100 \%$ ) $3,58 \mathrm{~mm}$

Tempo por giro (relê a $100 \%$ ) ............9,55 h

Vazão total $245,19 \mathrm{~m}^{3} / \mathrm{h}$

Vazão por área $3,75 \mathrm{~m}^{3} / \mathrm{h}$.ha

Comprimento até a última torre $437,57 \mathrm{~m}$

Comprimento da tubulação $456,20 \mathrm{~m}$

Raio total irrigado $456,20 \mathrm{~m}$

Carga estática no final da tubulação $\ldots .20 \mathrm{~m}$

Motorredutores em alta 6

Motorredutores em baixa 3

\section{Adutora:}

Tubulação de aço zincado de $250 \mathrm{~mm}$ de diâmetro com $252 \mathrm{~m}$ de comprimento (a)

Perda de carga em $100 \mathrm{~m}$ $0,8286 \mathrm{~m}$

Perda de carga na tubulação (a)......2,0882 m

Tubulação de aço zincado de $200 \mathrm{~mm}$ de diâmetro com $300 \mathrm{~m}$ de comprimento (b)

Perda de carga em $100 \mathrm{~m}$ $2,5057 \mathrm{~m}$

Perda de carga na tubulação (b) .....7,5171 m

Perda de carga total $(a+b)$ $9,6053 \mathrm{~m}$

\section{Composição da motobomba:}

\subsection{Cálculo da altura total:}

Carga estática no final da tubulação $20,00 \mathrm{~m}$

Desnivel entre o centro do pivô e o ponto mais alto $10,00 \mathrm{~m}$

Perda de carga na tubulação do pivô $15,35 \mathrm{~m}$

Altura dos aspersores $3,50 \mathrm{~m}$

Carga estática no ponto pivô $48,85 \mathrm{~m}$

Desnivel entre a motobomba e o centro do pivô $18,00 \mathrm{~m}$

Perdas na adutora $9,61 \mathrm{~m}$

Altura máxima de sucção prevista $3,00 \mathrm{~m}$

Perdas localizadas $5,00 \mathrm{~m}$

Altura manométrica total $84,46 \mathrm{~m}$ 
4.2. Dados da bomba centrífuga:

Marca IMBIL

Modelo. ITA $125-500 / 2$

Estágios 2

Diâmetro do rotor $335,00 \mathrm{~mm}$

Potência no eixo $104,13 \mathrm{cv}$

Consumo 19,84 litros/h

Velocidade $1750 \mathrm{rpm}$

Vazão $246,00 \mathrm{~m}^{3} / \mathrm{h}$

Altura manométrica $88 \mathrm{~m}$

Rendimento $77 \%$

Potência máxima $124,95 \mathrm{cv}$

NPSH requerido $2,60 \mathrm{~m}$

NPSH disponivel $5,31 \mathrm{~m}$

4.3. Dados gerais do motor diesel:

Marca MWM

Modelo. TD 229

Número de cilindros ....6 6

Potência $112 \mathrm{cv}$

Rotação de trabalho. $1750 \mathrm{rpm}$ 
APÊNDICE 4. Custos e composição do sistema a óleo diesel (D) para os tipos Comercial/Fabricante; Teórico/Econômico; Comercial/Teórico/Econômico; e Comercial/Econômico.

\section{Custos}

1.1. Custos de aquisição e custos fixos anual por item do sistema (USS):

\begin{tabular}{|c|c|c|c|c|}
\hline Item & \multicolumn{4}{|c|}{ Custo de Aquisição } \\
\cline { 2 - 5 } Sistema & Com./Fabr. & Teór./Econ. & Com./Teór./Econ. & Com./Econ. \\
\hline Tubulação de sucção & 927,00 & $\mathbf{8 5 8 , 2 8}$ & $\mathbf{8 5 8 , 2 8}$ & $\mathbf{9 2 7 , 0 0}$ \\
Tubulação de recalque & $14.549,44$ & $20.908,54$ & $20.869,17$ & $20.482,74$ \\
Bomba hidráulica & $10.126,64$ & $9.367,60$ & $9.368,49$ & $10.126,64$ \\
Motor diesel e acessór. & $32.516,70$ & $30.803,81$ & $30.807,90$ & $32.516,70$ \\
Obras de constr. civil & $4.526,34$ & 4.526 .34 & 4.526 .34 & $4.526,34$ \\
\hline Total & $\mathbf{6 2 . 6 4 6 , 1 2}$ & $\mathbf{6 6 . 4 6 4 , 5 7}$ & $\mathbf{6 6 . 4 3 0 , 1 8}$ & $\mathbf{6 8 . 5 7 9 , 4 2}$ \\
\hline
\end{tabular}

1.2. Custos fixos anual do sistema (US\$):

- Com./Fabr. $6.417,73$

- Teór./Econ. $6.808,91$

- Com./Teór./Econ. $6.805,39$

- Com./Econ. $7.025,57$

1.3. Custos anual de bombeamento do sistema (US\$):

- Com./Fabr. $19.761,89$

- Teór./Econ. $16.963,24$

- Com./Teór./Econ. $16.966,99$

- Com./Econ. $16.927,71$

1.4. Custos anual com manutenção e reparos do sistema (US\$):

- Com./Fabr. $3.169,56$

- Teór./Econ. 3.008,14

- Com./Teór./Econ. 3.008,27

- Com./Econ. $3.205,15$

1.5. Custos anual total do sistema (US\$):

- Com./Fabr. $29.349,18$

- Teór./Econ. $26.780,29$

- Com./Teór./Econ. $26.780,64$

- Com./Econ. $27.158,43$ 


\section{Composição do sistema}

\begin{tabular}{|c|c|c|c|c|}
\hline $\begin{array}{l}\text { Item } \\
\text { sistema }\end{array}$ & Com./Fabr. & Teór./Econ. & Com./Teór./Econ. & Com./Econ. \\
\hline $\mathrm{Ts}$ & $\begin{array}{l}1 \text { válv. sucção } 300 \mathrm{~mm} ; \\
1 \text { curva } 90^{\circ} 300 \mathrm{~mm} ; 1 \\
\text { luva cònica excêntrica } \\
300 \times 200 \mathrm{~mm} ; 2 \text { tubos } \\
300 \mathrm{~mm} \text {. }\end{array}$ & $\begin{array}{l}1 \text { válv. sucção } 300 \mathrm{~mm} \text {; } \\
1 \text { curva } 90^{\circ} 300 \mathrm{~mm} ; 1 \\
\text { luva cônica excêntrica } \\
300 \mathrm{~mm} ; 2 \text { tubos } 300 \\
\mathrm{~mm} \text {. }\end{array}$ & $\begin{array}{l}1 \text { válv. sucção } 300 \mathrm{~mm} ; \\
1 \text { curva } 90^{\circ} 300 \mathrm{~mm} ; 1 \\
\text { luva cônica excêntrica } \\
300 \mathrm{~mm} ; 2 \text { tubos } 300 \\
\mathrm{~mm} \text {. }\end{array}$ & $\begin{array}{l}1 \text { válv. sucção } 300 \mathrm{~mm} \text {; } \\
1 \text { curva } 90^{\circ} 300 \mathrm{~mm} ; 1 \\
\text { luva cônica excêntrica } \\
300 \times 200 \mathrm{~mm} ; 2 \text { tubos } \\
300 \mathrm{~mm} \text {. }\end{array}$ \\
\hline $\mathrm{Tr}$ & $\begin{array}{l}1 \text { luva cônica conc. } 250 \\
\times 150 \mathrm{~mm} ; 1 \mathrm{válvula} \\
\text { retenção } 250 \mathrm{~mm} ; 1 \\
\text { válvula gaveta } 250 \mathrm{~mm} ; \\
2 \text { curvas } 60^{\circ} 250 \mathrm{~mm} ; 1 \\
\text { curva } 90^{\circ} 250 \mathrm{~mm} ; 1 \\
\text { curva } 90^{\circ} 200 \mathrm{~mm} ; 42 \\
\text { tubos } 250 \mathrm{~mm} ; 50 \text { tubos } \\
200 \mathrm{~mm} \text {. }\end{array}$ & $\begin{array}{l}1 \text { luva cônica conc. } \\
300,4 \mathrm{~mm} ; 1 \text { válvula } \\
\text { retenção } 300,4 \mathrm{~mm} ; 1 \\
\text { válv. gaveta } 300,4 \mathrm{~mm} ; \\
2 \text { curva } 60^{\circ} 300,4 \mathrm{~mm} ; \\
2 \text { curvas } 90^{\circ} 300,4 \mathrm{~mm} ; \\
92 \text { tubos } 300,4 \mathrm{~mm} .\end{array}$ & $\begin{array}{l}1 \text { luva cônica conc. } 300 \\
\text { mm; 1 válvula retenção } \\
300 \mathrm{~mm} ; 1 \text { válv. gaveta } \\
300 \mathrm{~mm} ; 2 \text { curva } 60^{\circ} \\
300 \mathrm{~mm} ; 2 \text { curvas } 90^{\circ} \\
300 \mathrm{~mm} ; 92 \text { tubos } 300 \\
\mathrm{~mm} .\end{array}$ & $\begin{array}{l}1 \text { luva cônica conc. } 300 \\
x \quad 150 \mathrm{~mm} ; 1 \text { válvula } \\
\text { retenção } 300 \mathrm{~mm} ; 1 \\
\text { válv. gaveta } 300 \mathrm{~mm} ; 2 \\
\text { curvas } 60^{\circ} 300 \mathrm{~mm} ; 2 \\
\text { curvas } 90^{\circ} 300 \mathrm{~mm} ; 92 \\
\text { tubos } 300 \mathrm{~mm} .\end{array}$ \\
\hline $\mathrm{BH}$ & $\begin{array}{l}1 \text { bomba multiestágio, } \\
76,7 \mathrm{cv}, 125 / 2 \text {, rotor } \\
320 \mathrm{~mm} \text {, sucção } 200 \\
\text { mm, recalque } 150 \mathrm{~mm}, \\
\text { rendimento } 78 \% \text { (real). }\end{array}$ & $\begin{array}{l}1 \text { bomba multiestágio, } \\
65,8 \quad \mathrm{cv} \text {, rendimento } \\
78 \% \text { (modelo). }\end{array}$ & $\begin{array}{l}1 \text { bomba multiestágio, } \\
65,8 \mathrm{cv} \text {, rendimento } \\
78 \% \text { (modelo). }\end{array}$ & $\begin{array}{l}1 \text { bomba multiestágio, } \\
65,8 \mathrm{cv}, 125 / 2 \text {, rotor } \\
295 \mathrm{~mm} \text {, sucção } 200 \\
\text { mm, recalque } 150 \mathrm{~mm} \text {, } \\
\text { rendimento } 78 \% \text { (real). }\end{array}$ \\
\hline $\mathrm{MCA}$ & $\begin{array}{l}1 \text { motor diesel MWM, } \\
\text { TD } 229-6,112 \mathrm{cv} \text {. }\end{array}$ & $\begin{array}{l}1 \text { motor diesel MWM, } \\
92,8 \mathrm{cv} \text {. }\end{array}$ & $\begin{array}{l}1 \text { motor diesel MWM, } \\
92,8 \mathrm{cv} \text {. }\end{array}$ & $\begin{array}{l}1 \text { motor diesel MWM, } \\
\text { TD } 229-6,112 \mathrm{cv} \text {. }\end{array}$ \\
\hline $\mathrm{OCC}$ & $\begin{array}{l}1 \text { casa de bombas de } \\
14,00 \mathrm{~m}^{2} \text {, acabamento } \\
\text { rústico. }\end{array}$ & $\begin{array}{l}1 \text { casa de bombas de } \\
14,00 \mathrm{~m}^{2}, \text { acabamento } \\
\text { nústico. }\end{array}$ & $\begin{array}{l}1 \text { casa de bombas de } \\
14,00 \mathrm{~m}^{2} \text {, acabamento } \\
\text { nustico. }\end{array}$ & $\begin{array}{l}1 \text { casa de bombas de } \\
14,00 \mathrm{~m}^{2} \text {, acabamento } \\
\text { rústico. }\end{array}$ \\
\hline
\end{tabular}


APÊNDICE 5. Custos e composição do sistema à eletricidade na tarifa convencional normal (ECN) para os tipos Comercial/Fabricante; Teórico/Econômico; Comercial/Teórico/Econômico; e Comercial/Econômico.

\section{Custos}

1.1. Custos de aquisição por item do sistema (US\$):

\begin{tabular}{|c|c|c|c|c|}
\hline \multirow{2}{*}{ Item do } & \multicolumn{4}{|c|}{ Custo de Aquisição } \\
\cline { 2 - 5 } Sistema & Com./Fabr. & Teór./Econ. & Com./Teór./Econ. & Com./Econ. \\
\hline Tubulação de sucção & 927.00 & 858,28 & 858,28 & 927,00 \\
Tubulação de recalque & 14.549 .44 & $19.132,87$ & $20.869,17$ & $20.482,74$ \\
Bomba hidráulica & 10.126 .64 & $9.416,46$ & $9.368,49$ & 10.126 .64 \\
Motor elétrico & 4.580 .68 & $3.099,35$ & $3.065,26$ & $3.091,97$ \\
Banco de capacitores & 246.83 & 182,76 & 181,27 & 273,62 \\
Chave de partida & $6.294,46$ & $5.531,71$ & $5.487,32$ & $4.946,30$ \\
Transformador elétrico & $3.236,00$ & $2.466,64$ & $2.447,23$ & $2.800,00$ \\
Acessórios elétricos & $1.456,20$ & $1.109,99$ & $1.101,25$ & $1.260,00$ \\
Linha de alta tensão & 13.680 .00 & $13.680,00$ & $13.680,00$ & $13.680,00$ \\
Obras de constr. civil & 4.526 .34 & $4.526,34$ & $4.526,34$ & $4.526,34$ \\
\hline Total & $\mathbf{5 9 . 6 2 3 , 5 9}$ & $\mathbf{6 0 . 0 0 4 , 4 1}$ & $\mathbf{6 1 . 5 8 4 , 6 3}$ & $\mathbf{6 2 . 1 1 4 , 6 1}$ \\
\hline
\end{tabular}

1.2. Custos fixos anual do sistema (US\$):

- Com./Fabr. $6.108,09$

- Teór./Econ. $6.147,11$

- Com./Teór./Econ. $6.308,99$

- Com./Econ. $6.363,28$

1.3. Custos anual de bombeamento do sistema (US\$):

\begin{tabular}{|c|c|c|c|c|}
\hline Faturamento & Com./Fabr. & Teór./Econ. & Com./Teór./Econ. & Com./Econ. \\
\hline FD & $2.318,87$ & $2.082,16$ & $2.057,47$ & $1.989,64$ \\
FC & 9.718 .29 & $\mathbf{8 . 7 2 6 . 2 5}$ & $\mathbf{8 . 6 2 2 , 7 9}$ & $\mathbf{8 . 3 3 8 . 5 2}$ \\
\hline Total & $\mathbf{1 2 . 0 3 7 , 1 6}$ & $\mathbf{1 0 . 8 0 8 , 4 0}$ & $\mathbf{1 0 . 6 8 0 , 2 6}$ & $\mathbf{1 0 . 3 2 8 , 1 6}$ \\
\hline
\end{tabular}

1.4. Custos anual com manutenção e reparos do sistema (US\$):

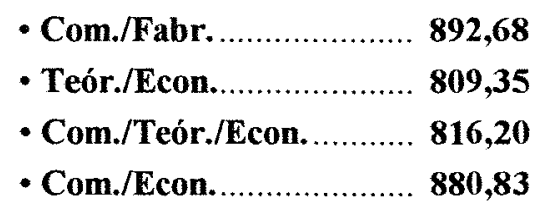




\subsection{Custos anual total do sistema (US\$):}

- Com./Fabr.

$19.037,93$

- Teór./Econ.

$17.764,86$

- Com./Teór./Econ.

$17.805,45$

- Com./Econ.

$17.572,27$

\section{Composição do sistema}

\begin{tabular}{|c|c|c|c|c|}
\hline $\begin{array}{l}\text { Item } \\
\text { sistema }\end{array}$ & Com./Fabr. & Teór/Econ. & Com./Teór./Econ. & Com.Econ. \\
\hline$T_{s}$ & $\begin{array}{l}\text { válv. sucção } 300 \mathrm{~mm} ; \\
\text { curva } 90^{\circ} 300 \mathrm{~mm} ; 1 \\
\text { va cônica excêntrica } \\
0 \times 200 \mathrm{~mm} ; 2 \text { tubos } \\
0 \mathrm{~mm} .\end{array}$ & $\begin{array}{l}1 \text { válv. sucção } 300 \mathrm{~mm} ; \\
1 \text { curva } 90^{\circ} 300 \mathrm{~mm} ; 1 \\
\text { luva cônica excêntrica } \\
300 \mathrm{~mm} ; 2 \text { tubos } 300 \\
\mathrm{~mm} .\end{array}$ & $\begin{array}{l}1 \text { válv. sucção } 300 \mathrm{~mm} ; \\
1 \text { curva } 90^{\circ} \quad 300 \mathrm{~mm} ; 1 \\
\text { luva cônica excêntrica } \\
300 \mathrm{~mm} ; 2 \text { tubos } 300 \\
\text { mm. }\end{array}$ & $\begin{array}{l}1 \text { válv. sucção } 300 \mathrm{~mm} ; \\
1 \text { curva } 90^{\circ} 300 \mathrm{~mm} ; 1 \\
\text { luva cônica excêntrica } \\
300 \times 200 \mathrm{~mm} ; 2 \text { tubos } \\
300 \mathrm{~mm} \text {. }\end{array}$ \\
\hline $\mathrm{Tr}$ & $\begin{array}{l}1 \text { luva cônica conc. } 250 \\
\times 150 \mathrm{~mm} ; 1 \text { válvula } \\
\text { retenção } 250 \mathrm{~mm} ; 1 \\
\text { válvula gaveta } 250 \mathrm{~mm} ; \\
2 \text { curvas } 60^{\circ} 250 \mathrm{~mm} ; 1 \\
\text { curva } 90^{\circ} 250 \mathrm{~mm} ; 1 \\
\text { curva } 90^{\circ} 200 \mathrm{~mm} ; 42 \\
\text { tubos } 250 \mathrm{~mm} ; 50 \text { tubos } \\
200 \mathrm{~mm} . \\
\end{array}$ & $\begin{array}{l}1 \text { luva cônica conc. } 281 \\
\text { mm; } 1 \text { válvula retenção } \\
281 \mathrm{~mm} \text {; } \text { válv. gaveta } \\
281 \mathrm{~mm} ; 2 \text { curva } 60^{\circ} \\
281 \mathrm{~mm} ; 2 \text { curvas } 90^{\circ} \\
281 \mathrm{~mm} ; 92 \text { tubos } 281 \\
\mathrm{~mm} .\end{array}$ & $\begin{array}{l}1 \text { luva cônica conc. } 300 \\
\text { mm; } 1 \text { válvula retenção } \\
300 \mathrm{~mm} ; 1 \text { válv. gaveta } \\
300 \mathrm{~mm} ; 2 \text { curva } 60^{\circ} \\
300 \mathrm{~mm} ; 2 \text { curvas } 90^{\circ} \\
300 \mathrm{~mm} ; 92 \text { tubos } 300 \\
\mathrm{~mm} .\end{array}$ & $\begin{array}{l}1 \text { luva cônica conc. } 300 \\
\times 150 \mathrm{~mm} ; 1 \mathrm{dálvula} \\
\text { retenção } 300 \mathrm{~mm} ; 1 \\
\text { válv. gaveta } 300 \mathrm{~mm} ; 2 \\
\text { curvas } 60^{\circ} 300 \mathrm{~mm} ; 2 \\
\text { curvas } 90^{\circ} 300 \mathrm{~mm} ; 92 \\
\text { tubos } 300 \mathrm{~mm} \text {. }\end{array}$ \\
\hline $\mathrm{BH}$ & 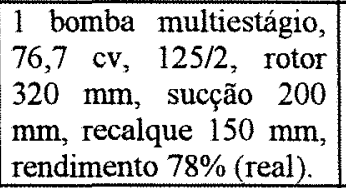 & $\begin{array}{l}1 \text { bomba multiestágio, } \\
66,6 \mathrm{cv}, \quad \text { rendimento } \\
78 \% \text { (modelo). }\end{array}$ & $\begin{array}{l}1 \text { bomba multiestágio, } \\
65,8 \quad \mathrm{cv} \text {, rendimento } \\
78 \% \text { (modelo). }\end{array}$ & $\begin{array}{l}1 \text { bomba multiestágio, } \\
65,8 \mathrm{cv}, 125 / 2 \text {, rotor } \\
295 \mathrm{~mm} \text {, sucção } 200 \\
\text { mm, recalque } 150 \mathrm{~mm} \text {, } \\
\text { rendimento } 78 \% \text { (real). }\end{array}$ \\
\hline$\overline{\mathrm{ME}}$ & $\begin{array}{l}1 \text { motor trifásico } 60 \mathrm{~Hz} \\
1800 \mathrm{rpm}, 380 \mathrm{~V} \text {, classe } \\
\text { "B", } 125 \mathrm{cv} \text {, rendimen- } \\
\text { to } 91 \% \text { (real), cos } 0,87 \\
\text { (real). }\end{array}$ & $\begin{array}{l}1 \text { motor trifásico } 60 \mathrm{~Hz}, \\
1800 \mathrm{mpm}, 380 \mathrm{~V} \text {, classe } \\
\text { " } \mathrm{B} \text { ", } 93,9 \mathrm{cv}, \text { rendimen- } \\
\text { to } 88 \% \text { (modelo), cos } \varphi \\
0,87 \text { (modelo). } \\
\end{array}$ & $\begin{array}{l}1 \text { motor trifásico } 60 \mathrm{~Hz}, \\
1800 \mathrm{rpm}, 380 \mathrm{~V} \text {, classe } \\
\text { " } \mathrm{B} \text { ", } 92,8 \mathrm{cv}, \text { rendimen- } \\
\text { to } 88 \% \text { (modelo), cos } \varphi \\
0,87 \text { (modelo). }\end{array}$ & $\begin{array}{l}1 \text { motor trifásico } 60 \mathrm{~Hz} \text {, } \\
1800 \mathrm{rpm}, 220 / 380 \mathrm{~V}, \\
\text { classe "B", } 100 \mathrm{cv}, \\
\text { rendimento } 91 \% \text { (real), } \\
\cos \varphi 0,85 \text { (real). }\end{array}$ \\
\hline $\mathrm{BC}$ & $\begin{array}{l}1 \text { banco capac. trifásico } \\
380 \mathrm{~V}, 17.500 \mathrm{VAr} \text {. }\end{array}$ & $\begin{array}{l}\text { trifásico } \\
\text { VAr. }\end{array}$ & $\begin{array}{l}\text { trifásico } \\
\text { VAr. }\end{array}$ & $\begin{array}{l}\text { trifásico } \\
\text { VAr. }\end{array}$ \\
\hline CP & $\begin{array}{l}1 \text { ch. partida compensa- } \\
\text { dora } 380 \mathrm{~V} \text { (triângulo), } \\
125 \mathrm{cv} \text {. }\end{array}$ & $\begin{array}{l}80 \mathrm{~V} \text { (triângulo), } \\
8\end{array}$ & $\begin{array}{l}\text { mpensa- } \\
\text { iângulo), }\end{array}$ & \begin{tabular}{|l}
$1 \mathrm{ch} . \mathrm{pe}$ \\
dora 38 \\
$100 \mathrm{cv}$ \\
\end{tabular} \\
\hline $\mathrm{TE}$ & \begin{tabular}{|l|} 
transformador elétrico \\
trifäsico classe $15 \mathrm{kV}$, \\
$150.000 \mathrm{VA}$.
\end{tabular} & $\begin{array}{l}1 \text { transformador elétrico } \\
\text { trifäsico classe } 15 \mathrm{kV} \text {, } \\
90.200 \text { VA. } \\
\end{array}$ & $\begin{array}{l}\text { transformador elétrico } \\
\text { trifásico classe } 15 \mathrm{kV}, \\
89.100 \text { VA. } \\
\end{array}$ & $\begin{array}{l}\text { sformador elétrico } \\
\text { co classe } 15 \mathrm{kV} \text {, } \\
00 \text { VA. }\end{array}$ \\
\hline $\mathrm{AE}$ & \begin{tabular}{lr|} 
acessórios & elétricos: \\
isoladores; & eletrodutos; \\
conectores; & hastes de \\
aterramento; cabos; etc.
\end{tabular} & $\begin{array}{lr}\text { acessórios } & \text { elétricos: } \\
\text { isoladores; } & \text { eletrodutos; } \\
\text { conectores; } & \text { hastes de } \\
\text { aterramento; cabos; etc. }\end{array}$ & 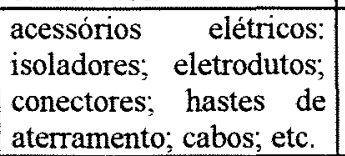 & $\begin{array}{l}\text { acessórios elétricos: } \\
\text { isoladores; eletrodutos; } \\
\text { conectores; hastes de } \\
\text { aterramento; cabos; etc. }\end{array}$ \\
\hline LAT & $\begin{array}{l}1 \text { linha de alta tensão de } \\
1800 \mathrm{~m} \text {, padrão CPFL. }\end{array}$ & & & \\
\hline OCC & $\begin{array}{l}1 \text { casa de bombas de } \\
14,00 \mathrm{~m}^{2}, \\
\text { rustico. }\end{array}$ & \begin{tabular}{ll|}
1 casa de & bombas de \\
$14,00 \mathrm{~m}^{2}$, & acabamento \\
rústico.
\end{tabular} & $\begin{array}{l}1 \text { casa de bombas de } \\
14,00 \mathrm{~m}^{2} \text {, acabamento } \\
\text { rústico. }\end{array}$ & $\begin{array}{l}1 \text { casa de bombas de } \\
14,00 \mathrm{~m}^{2} \text {, acabamento } \\
\text { rústico. }\end{array}$ \\
\hline
\end{tabular}


APENDICE 6. Custos e composição do sistema à eletricidade na tarifa convencional irrigante (ECI) para os tipos Comercial/Fabricante; Teórico/Eco-nômico; Comercial/Teórico/Econômico; e Comercial/Fconômico.

\section{Custos}

1.1. Custos de aquisição por item do sistema (US\$):

\begin{tabular}{|c|c|c|c|c|}
\hline Item do & \multicolumn{3}{|c|}{ Custo de Aquisição } \\
\cline { 2 - 5 } Sistema & Com./Fabr. & Teór./Econ. & Com./Teór./Econ. & Com./Econ. \\
\hline Tubulação de sucção & 927,00 & 858,28 & 858,28 & 927,00 \\
Tubulação de recalque & $14.549,44$ & $18.339,60$ & $16.336,46$ & $15.899,44$ \\
Bomba hidráulica & $10.126,64$ & $9.445,69$ & $9.551,40$ & $10.126,64$ \\
Motor elétrico & $4.580,68$ & $3.120,23$ & $3.196,33$ & $3.091,97$ \\
Banco de capacitores & 246,83 & 183,67 & 186,97 & 273,62 \\
Chave de partida & $6.294,46$ & $5.558,83$ & $5.657,29$ & $4.946,30$ \\
Transformador elétrico & $3.236,00$ & $2.478,50$ & $2.521,54$ & $2.800,00$ \\
Acessórios elétricos & $1.456,20$ & $1.115,32$ & $1.134,69$ & $1.260,00$ \\
Linha de alta tensão & $13.680,00$ & $13.680,00$ & $13.680,00$ & $13.680,00$ \\
Obras de constr. civil & $4.526,34$ & $4.526,34$ & $4.526,34$ & $4.526,34$ \\
\hline Total & $\mathbf{5 9 . 6 2 3 , 5 9}$ & $\mathbf{5 9 . 3 0 6 , 4 7}$ & $\mathbf{5 7 . 6 4 9 , 3 1}$ & $\mathbf{5 7 . 5 3 1 , 3 1}$ \\
\hline
\end{tabular}

1.2. Custos fixos anual do sistema (US\$):
- Com./Fabr.
$6.108,09$
- Teór./Econ. $6.075,60$
- Com./Teór./Econ. $5.905,84$
- Com./Econ. $5.893,75$

1.3. Custos anual de bombeamento do sistema (US\$):

\begin{tabular}{|c|c|c|c|c|}
\hline Faturamento & Com./Fabr. & Teór./Econ. & Com./Téor./Econ. & Com./Econ. \\
\hline FD & $2.318,87$ & $2.097,29$ & $2.152,51$ & $2.081,54$ \\
FCahc & $6.802,79$ & $6.152,75$ & $6.314,74$ & $6.106,56$ \\
FCahe & $\mathbf{8 7 4 , 6 5}$ & 791,07 & 811,90 & $\mathbf{7 8 5 , 1 3}$ \\
\hline Total & $\mathbf{9 . 9 9 6 , 3 1}$ & $\mathbf{9 . 0 4 1 , 1 0}$ & $\mathbf{9 . 2 7 9 , 1 5}$ & $\mathbf{8 . 9 7 3 , 2 3}$ \\
\hline
\end{tabular}

1.4. Custos anual com manutenção e reparos do sistema (US\$):
- Com./Fabr. 892,68
- Teór./Econ. 806,76
- Com./Teór./Econ. 802,63
- Com./Econ. 853,33 


\subsection{Custos anual total do sistema (US\$): \\ - Com./Fabr. $16.548,29$ \\ - Teór./Econ. $15.923,47$ \\ - Com./Teór./Econ. \\ $15.987,61$ \\ - Com./Econ. $15.720,31$}

\section{Composição do sistema}

\begin{tabular}{|c|c|c|c|c|}
\hline $\begin{array}{l}\text { Item } \\
\text { sistema }\end{array}$ & Com./Fabr. & Teór./Econ. & Com.Teór./Econ. & Com./Econ. \\
\hline $\mathrm{Ts}$ & $\begin{array}{l}1 \text { válv. sucção } 300 \mathrm{~mm} \\
1 \text { curva } 90^{\circ} 300 \mathrm{~mm} ; 1 \\
\text { luva cônica excêntrica } \\
300 \times 200 \mathrm{~mm} ; 2 \text { tubos } \\
300 \mathrm{~mm} \text {. }\end{array}$ & $\begin{array}{l}1 \text { válv. sucção } 300 \mathrm{~mm} ; \\
1 \text { curva } 90^{\circ} 300 \mathrm{~mm} ; 1 \\
\text { luva cônica excêntrica } \\
300 \mathrm{~mm} ; 2 \text { tubos } 300 \\
\mathrm{~mm} \text {. }\end{array}$ & $\begin{array}{l}1 \text { válv. sucção } 300 \mathrm{~mm} \text {; } \\
1 \text { curva } 90^{\circ} 300 \mathrm{~mm} ; 1 \\
\text { luva cônica excêntrica } \\
300 \mathrm{~mm} ; 2 \text { tubos } 300 \\
\text { mm. }\end{array}$ & $\begin{array}{l}1 \text { válv. sucção } 300 \mathrm{~mm} \text {; } \\
1 \text { curva } 90^{\circ} 300 \mathrm{~mm} ; 1 \\
\text { luva cônica excêntrica } \\
300 \times 200 \mathrm{~mm} ; 2 \text { tubos } \\
300 \mathrm{~mm} \text {. }\end{array}$ \\
\hline $\mathrm{Tr}$ & 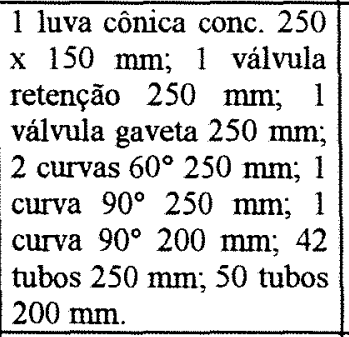 & $\begin{array}{l}1 \text { luva cônica conc. } 272 \\
\text { mm; } 1 \text { válvula retenção } \\
272 \mathrm{~mm} ; 1 \text { válv. gaveta } \\
272 \mathrm{~mm} ; 2 \text { curva } 60^{\circ} \\
272 \mathrm{~mm} ; 2 \text { curvas } 90^{\circ} \\
272 \mathrm{~mm} ; 92 \text { tubos } 272 \\
\mathrm{~mm} .\end{array}$ & $\begin{array}{l}\text { luva cônica conc. } 250 \\
\mathrm{~mm} ; 1 \text { válvula retenção } \\
250 \mathrm{~mm} ; 1 \text { válv. gaveta } \\
250 \mathrm{~mm} ; 2 \text { curva } 60^{\circ} \\
250 \mathrm{~mm} ; 2 \text { curvas } 90^{\circ} \\
250 \mathrm{~mm} ; 92 \text { tubos } 250 \\
\mathrm{~mm} .\end{array}$ & $\begin{array}{l}1 \text { luva cônica conc. } 250 \\
\text { X } 150 \mathrm{~mm} ; 1 \text { válvula } \\
\text { retenção } 250 \mathrm{~mm} ; 1 \\
\text { válv. gaveta } 250 \mathrm{~mm} ; 2 \\
\text { curvas } 60^{\circ} 250 \mathrm{~mm} ; 2 \\
\text { curvas } 90^{\circ} 250 \mathrm{~mm} ; 92 \\
\text { tubos } 250 \mathrm{~mm} \text {. }\end{array}$ \\
\hline $\mathrm{BH}$ & $\begin{array}{l}1 \text { bomba multiestágio, } \\
76,7 \mathrm{cv}, 125 / 2 \text {, rotor } \\
320 \mathrm{~mm} \text {, sucção } 200 \\
\mathrm{~mm} \text {, recalque } 150 \mathrm{~mm} \text {, } \\
\text { rendimento } 78 \% \text { (real). }\end{array}$ & $\begin{array}{l}1 \text { bomba multiestágio, } \\
67,1 \quad \mathrm{cv} \text {, rendimento } \\
78 \% \text { (modelo). }\end{array}$ & $\begin{array}{l}1 \text { bomba multiestágio, } \\
68,8 \quad \mathrm{cv} \text {, rendimento } \\
78 \% \text { (modelo). }\end{array}$ & $\begin{array}{l}1 \text { bomba multiestágio, } \\
68,8 \mathrm{cv}, 125 / 2 \text {, rotor } \\
320 \mathrm{~mm} \text {, sucção } 200 \\
\text { mm, recalque } 150 \mathrm{~mm} \text {, } \\
\text { rendimento } 78 \% \text { (real). }\end{array}$ \\
\hline $\mathrm{ME}$ & $\begin{array}{l}1 \text { motor trifásico } 60 \mathrm{~Hz} \text {, } \\
1800 \mathrm{rpm}, 380 \mathrm{~V} \text {, classe } \\
\text { "B", } 125 \mathrm{cv} \text {, rendimen- } \\
\text { to } 91 \% \text { (real), cos } \varphi 0,87 \\
\text { (real). }\end{array}$ & $\begin{array}{l}1 \text { motor trifásico } 60 \mathrm{~Hz} \text {, } \\
1800 \mathrm{rpm}, 380 \mathrm{~V} \text {, classe } \\
\text { " } \mathrm{B} \text { ", } 94,6 \mathrm{cv} \text {, rendimen- } \\
\text { to } 88 \% \text { (modelo), cos } \varphi \\
0,87 \text { (modelo). }\end{array}$ & $\begin{array}{l}1 \text { motor trifásico } 60 \mathrm{~Hz}, \\
1800 \mathrm{rpm}, 380 \mathrm{~V} \text {, classe } \\
\text { " } \mathrm{B} ", 97,1 \mathrm{cv}, \text { rendimen- } \\
\text { to } 88 \% \text { (modelo), cos } \varphi \\
0,87 \text { (modelo). }\end{array}$ & $\begin{array}{l}1 \text { motor trifásico } 60 \mathrm{~Hz} \text {, } \\
1800 \mathrm{rpm}, 220 / 380 \mathrm{~V} \text {, } \\
\text { classe "B", } 100 \mathrm{cv} \text {, } \\
\text { rendimento } 91 \% \text { (real), } \\
\cos \varphi 0,85 \text { (real). }\end{array}$ \\
\hline $\mathrm{BC}$ & $\begin{array}{l}1 \text { banco capac. trifásico } \\
380 \mathrm{~V}, 17.500 \mathrm{VAr} \text {. }\end{array}$ & $\begin{array}{l}1 \text { banco capac. trifásico } \\
380 \mathrm{~V}, 9.800 \mathrm{VAr} \text {. }\end{array}$ & $\begin{array}{l}\text { ac. trifásico } \\
\text { OO VAr. }\end{array}$ & $\begin{array}{l}1 \text { banco capac. trifásico } \\
380 \mathrm{~V}, 20.000 \mathrm{VAr} \text {. }\end{array}$ \\
\hline $\mathrm{CP}$ & $\begin{array}{l}1 \mathrm{ch} \text {. partida compensa- } \\
\text { dora } 380 \mathrm{~V} \text { (triângulo), } \\
125 \mathrm{cv} \text {. }\end{array}$ & $\begin{array}{l}1 \mathrm{ch} \text { partida compensa- } \\
\text { dora } 380 \mathrm{~V} \text { (triângulo), } \\
94,6 \mathrm{cv} \text {. }\end{array}$ & $\begin{array}{l}1 \mathrm{ch} \text { partida compensa- } \\
\text { dora } 380 \mathrm{~V} \text { (triângulo), } \\
97,1 \mathrm{cv} \text {. }\end{array}$ & $\begin{array}{l}1 \text { ch. partida compensa- } \\
\text { dora } 380 \mathrm{~V} \text { (triângulo), } \\
100 \mathrm{cv} \text {. }\end{array}$ \\
\hline $\mathrm{TE}$ & $\begin{array}{l}1 \text { transformador elétrico } \\
\text { trifásico classe } 15 \mathrm{kV} \text {, } \\
150.000 \mathrm{VA} \text {. }\end{array}$ & $\begin{array}{l}1 \text { transformador elétrico } \\
\text { trifásico classe } 15 \mathrm{kV} \text {, } \\
90.800 \text { VA. }\end{array}$ & $\begin{array}{l}1 \text { transformador elétrico } \\
\text { trifásico classe } 15 \mathrm{kV} \text {, } \\
93.200 \mathrm{VA} \text {. }\end{array}$ & $\begin{array}{l}1 \text { transformador elétrico } \\
\text { trifásico classe } 15 \mathrm{kV} \text {, } \\
112.500 \text { VA. } \\
\end{array}$ \\
\hline $\mathrm{AE}$ & 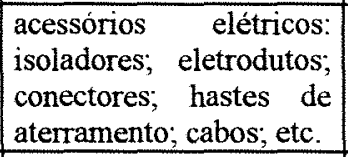 & $\begin{array}{lr}\text { acessórios } & \text { elétricos: } \\
\text { isoladores; } & \text { eletrodutos; } \\
\text { conectores; } & \text { hastes de } \\
\text { aterramento; cabos; etc. }\end{array}$ & $\begin{array}{l}\text { acessórios elétricos: } \\
\text { isoladores; eletrodutos; } \\
\text { conectores; hastes de } \\
\text { aterramento; cabos; etc. }\end{array}$ & $\begin{array}{l}\text { acessórios elétricos: } \\
\text { isoladores; eletrodutos; } \\
\text { conectores; hastes de } \\
\text { aterramento; cabos; etc. }\end{array}$ \\
\hline LAT & $\begin{array}{l}1 \text { linha de alta tensão de } \\
1800 \mathrm{~m} \text {, padrão CPFL. }\end{array}$ & $\begin{array}{l}1 \text { linha de alta tensão de } \\
1800 \mathrm{~m} \text {, padrão } \mathrm{CPFL} \text {. }\end{array}$ & $\begin{array}{l}1 \text { linha de alta tensão de } \\
1800 \mathrm{~m} \text {, padrão CPFL. }\end{array}$ & $\begin{array}{l}1 \text { linha de alta tensão de } \\
1800 \mathrm{~m} \text {, padrão CPFL. }\end{array}$ \\
\hline $\mathrm{OCC}$ & $\begin{array}{l}1 \text { casa de bombas de } \\
14,00 \mathrm{~m}^{2} \text {, acabamento } \\
\text { rústico. }\end{array}$ & $\begin{array}{l}1 \text { casa de bombas de } \\
14,00 \mathrm{~m}^{2}, \text { acabamento } \\
\text { rústico. }\end{array}$ & $\begin{array}{l}1 \text { casa de bombas de } \\
14,00 \mathrm{~m}^{2}, \text { acabamento } \\
\text { rústico. }\end{array}$ & $\begin{array}{l}1 \text { casa de bombas de } \\
14,00 \mathrm{~m}^{2} \text {, acabamento } \\
\text { rústico. }\end{array}$ \\
\hline
\end{tabular}


APÊNDICE 7. Custos e composição do sistema à eletricidade na tarifa verde normal (EVN) para os tipos Comercial/Fabricante; Teórico/Econômico; Comercial/Teórico/Econômico; e Comercial/Econômico.

1. Custos

1.1. Custos de aquisição por item do sistema (US\$):

\begin{tabular}{|c|c|c|c|c|}
\hline Item do & \multicolumn{4}{|c|}{ Custo de Aquisição } \\
\hline Sistema & Com./Fabr. & Teór./Econ. & Com./Teór./Econ. & Com./Econ. \\
\hline Tubulação de sucção & 927,00 & $\mathbf{8 5 8 , 2 8}$ & $\mathbf{8 5 8 , 2 8}$ & 927,00 \\
Tubulação de recalque & $14.549,44$ & $18.135,41$ & $16.336,46$ & $15.899,44$ \\
Bomba hidráulica & $10.126,64$ & $9.454,17$ & $9.551,40$ & $10.126,64$ \\
Motor elétrico & $4.580,68$ & $3.126,29$ & $3.196,33$ & $3.091,97$ \\
Banco de capacitores & 246,83 & 183,94 & 186,97 & 273,62 \\
Chave de partida & 6.294 .46 & $\mathbf{5 . 5 6 6 , 7 0}$ & $5.657,29$ & $4.946,30$ \\
Transformador elétrico & $3.236,00$ & $2.481,94$ & $2.521,54$ & $2.800,00$ \\
Acessórios elétricos & $1.456,20$ & $1.116,87$ & $1.134,69$ & $1.260,00$ \\
Linha de alta tensão & $13.680,00$ & $13.680,00$ & $13.680,00$ & $13.680,00$ \\
Obras de constr. civil & 4.526 .34 & $\mathbf{4 . 5 2 6 , 3 4}$ & $4.526,34$ & $4.526,34$ \\
\hline Total & $\mathbf{5 9 . 6 2 3 , 5 9}$ & $\mathbf{5 9 . 1 2 9 , 9 6}$ & $\mathbf{5 7 . 6 4 9 , 3 1}$ & $\mathbf{5 7 . 5 3 1 , 3 1}$ \\
\hline
\end{tabular}

1.2. Custos fixos anual do sistema (US\$):

- Com./Fabr. $6.108,09$

- Teór./Econ. $6.057,52$

- Com./Teór./Econ. $5.905,84$

- Com./Econ. $5.893,75$

1.3. Custos anual de bombeamento do sistema (US\$):

\begin{tabular}{|c|c|c|c|c|}
\hline Faturamento & Com./Fabr. & Teór./Econ. & Com./Teór./Econ. & Com./Econ. \\
\hline FD & $1.610,38$ & $1.459,02$ & $1.494,30$ & $1.444,51$ \\
FCsp & $1.395,11$ & $1.263,98$ & $1.294,55$ & $1.251,41$ \\
FCsfp & $3.957,20$ & $3.585,26$ & $3.671,95$ & $3.549,61$ \\
FCup & 457,41 & 414,42 & 424,44 & 410,30 \\
FCufp & $1.166,17$ & $1.056,56$ & $1.082,11$ & 1.046 .06 \\
\hline Total & $\mathbf{8 . 5 8 6 , 2 6}$ & $\mathbf{7 . 7 7 9 , 2 3}$ & $\mathbf{7 . 9 6 7 , 3 4}$ & $\mathbf{7 . 7 0 1 , 8 9}$ \\
\hline
\end{tabular}

1.4. Custos anual com manutenção e reparos do sistema (US\$):

- Com./Fabr. 892,68

- Teór./Econ. 806,17

- Com./Teór./Econ. 802,63

- Com./Econ. 853,33 


\subsection{Custos anual total do sistema (US\$):}

- Com./Fabr.

$15.587,03$

- Teór./Econ.

$14.614,93$

- Com./Teór./Econ.

$14.675,81$

- Com./Econ.

$14.448,97$

\section{Composição do sistema}

\begin{tabular}{|c|c|c|c|c|}
\hline $\begin{array}{l}\text { Item } \\
\text { sistema }\end{array}$ & Com. Fabr. & Teór./Econ. & Com./Teór./Econ. & Com./Econ. \\
\hline $\mathrm{Ts}$ & $\begin{array}{l}1 \text { válv. sucção } 300 \mathrm{~mm} \text {; } \\
1 \text { curva } 90^{\circ} 300 \mathrm{~mm} ; 1 \\
\text { luva cônica excêntrica } \\
300 \times 200 \mathrm{~mm} ; 2 \text { tubos } \\
300 \mathrm{~mm} \text {. }\end{array}$ & $\begin{array}{l}1 \text { válv sucção } 300 \mathrm{~mm} \text {; } \\
1 \text { curva } 90^{\circ} 300 \mathrm{~mm} ; 1 \\
\text { luva cônica excêntrica } \\
300 \mathrm{~mm} ; 2 \text { tubos } 300 \\
\text { mm. }\end{array}$ & $\begin{array}{l}1 \text { válv. sucção } 300 \mathrm{~mm} ; \\
1 \text { curva } 90^{\circ} 300 \mathrm{~mm} ; 1 \\
\text { luva cônica excêntrica } \\
300 \mathrm{~mm} ; 2 \text { tubos } 300 \\
\mathrm{~mm} \text {. }\end{array}$ & $\begin{array}{l}1 \text { válv. sucção } 300 \mathrm{~mm} \text {; } \\
1 \text { curva } 90^{\circ} 300 \mathrm{~mm} ; 1 \\
\text { luva cônica excêntrica } \\
300 \times 200 \mathrm{~mm} ; 2 \text { tubos } \\
300 \mathrm{~mm} \text {. }\end{array}$ \\
\hline $\operatorname{Tr}$ & $\begin{array}{l}1 \text { luva cônica conc. } 250 \\
\text { x } 150 \mathrm{~mm} ; 1 \text { válvula } \\
\text { retenção } 250 \mathrm{~mm} ; 1 \\
\text { válvula gaveta } 250 \mathrm{~mm} ; \\
2 \text { curvas } 60^{\circ} 250 \mathrm{~mm} ; 1 \\
\text { curva } 90^{\circ} 250 \mathrm{~mm} ; 1 \\
\text { curva } 90^{\circ} 200 \mathrm{~mm} ; 42 \\
\text { tubos } 250 \mathrm{~mm} ; 50 \text { tubos } \\
200 \mathrm{~mm} \text {. }\end{array}$ & $\begin{array}{l}1 \text { luva cônica conc. } 270 \\
\text { mm; } 1 \text { válvula retenção } \\
270 \mathrm{~mm} ; 1 \text { válv. gaveta } \\
270 \mathrm{~mm} ; 2 \text { curva } 60^{\circ} \\
270 \mathrm{~mm} ; 2 \text { curvas } 90^{\circ} \\
270 \mathrm{~mm} ; 92 \text { tubos } 270 \\
\mathrm{~mm} .\end{array}$ & $\begin{array}{l}1 \text { luva cônica conc. } 250 \\
\text { mm; } 1 \text { válvula retenção } \\
250 \mathrm{~mm} ; 1 \text { válv. gaveta } \\
250 \mathrm{~mm} ; 2 \text { curva } 60^{\circ} \\
250 \mathrm{~mm} ; 2 \text { curvas } 90^{\circ} \\
250 \mathrm{~mm} ; 92 \text { tubos } 250 \\
\mathrm{~mm} .\end{array}$ & $\begin{array}{l}1 \text { luva cônica conc. } 250 \\
\text { X } 150 \mathrm{~mm} ; 1 \text { válvula } \\
\text { retenção } 250 \mathrm{~mm} ; 1 \\
\text { válv. gaveta } 250 \mathrm{~mm} ; 2 \\
\text { curvas } 60^{\circ} 250 \mathrm{~mm} ; 2 \\
\text { curvas } 90^{\circ} 250 \mathrm{~mm} ; 92 \\
\text { tubos } 250 \mathrm{~mm} .\end{array}$ \\
\hline $\mathrm{BH}$ & $\begin{array}{l}1 \text { bomba multiestágio, } \\
76,7 \mathrm{cv} \text {, } 125 / 2 \text {, rotor } \\
320 \mathrm{~mm} \text {, sucção } 200 \\
\mathrm{~mm} \text {, recalque } 150 \mathrm{~mm} \text {, } \\
\text { rendimento } 78 \% \text { (real). }\end{array}$ & $\begin{array}{l}1 \text { bomba multiestágio, } \\
67,2 \mathrm{cv} \text {, rendimento } \\
78 \% \text { (modelo). }\end{array}$ & $\begin{array}{l}1 \text { bomba multiestágio, } \\
68,8 \mathrm{cv} \text {, rendimento } \\
78 \% \text { (modelo). }\end{array}$ & $\begin{array}{l}1 \text { bomba multiestágio, } \\
68,8 \mathrm{cv}, 125 / 2 \text {, rotor } \\
320 \mathrm{~mm} \text {, sucção } 200 \\
\text { mm, recalque } 150 \mathrm{~mm} \text {, } \\
\text { rendimento } 78 \% \text { (real). }\end{array}$ \\
\hline $\mathrm{ME}$ & $\begin{array}{l}1 \text { motor trifásico } 60 \mathrm{~Hz} \text {, } \\
1800 \mathrm{rpm}, 380 \mathrm{~V} \text {, classe } \\
\text { " } \mathrm{B} \text { ", } 125 \mathrm{cv} \text {, rendimen- } \\
\text { to } 91 \% \text { (real), cos } \varphi 0,87 \\
\text { (real). }\end{array}$ & $\begin{array}{l}1 \text { motor trifásico } 60 \mathrm{~Hz} \text {, } \\
1800 \mathrm{rpm}, 380 \mathrm{~V} \text {, classe } \\
\text { " } \mathrm{B} ", 94,8 \mathrm{cv} \text {, rendimen- } \\
\text { to } 88 \% \text { (modelo), cos } \varphi \\
0,87 \text { (modelo). }\end{array}$ & $\begin{array}{l}1 \text { motor trifásico } 60 \mathrm{~Hz}, \\
1800 \mathrm{rpm}, 380 \mathrm{~V} \text {, classe } \\
\text { " } \mathrm{B} ", 97,1 \mathrm{cv} \text {, rendimen- } \\
\text { to } 88 \% \text { (modelo), cos } \varphi \\
0,87 \text { (modelo). }\end{array}$ & $\begin{array}{l}1 \text { motor trifásico } 60 \mathrm{~Hz} \text {, } \\
1800 \mathrm{rpm}, 220 / 380 \mathrm{~V} \text {, } \\
\text { classe "B", } 100 \mathrm{cv} \text {, } \\
\text { rendimento } 91 \% \text { (real), } \\
\cos \varphi 0,85 \text { (real). }\end{array}$ \\
\hline $\mathrm{BC}$ & \begin{tabular}{|l|}
1 banco capac. trifásico \\
$380 \mathrm{~V}, 17.500 \mathrm{VAr}$. \\
\end{tabular} & $\begin{array}{l}1 \text { banco capac. trifásico } \\
380 \mathrm{~V}, 9.800 \mathrm{VAr} \text {. }\end{array}$ & $\begin{array}{l}1 \text { banco capac. trifásico } \\
380 \mathrm{~V}, 10.000 \text { VAr. } \\
\end{array}$ & $\begin{array}{l}1 \text { banco capac. trifásico } \\
380 \mathrm{~V}, 20.000 \mathrm{VAr} \text {. }\end{array}$ \\
\hline $\mathrm{CP}$ & $\begin{array}{l}1 \mathrm{ch} \text {. partida compensa- } \\
\text { dora } 380 \mathrm{~V} \text { (triângulo), } \\
125 \mathrm{cv} \text {. }\end{array}$ & $\begin{array}{l}\text { l ch. partida compensa- } \\
\text { dora } 380 \mathrm{~V} \text { (triângulo), } \\
94,8 \mathrm{cv} \text {. }\end{array}$ & $\begin{array}{l}1 \mathrm{ch} \text { partida compensa- } \\
\text { dora } 380 \mathrm{~V} \text { (triângulo), } \\
97,1 \mathrm{cv} \text {. }\end{array}$ & $\begin{array}{l}1 \mathrm{ch} \text { partida compensa- } \\
\text { dora } 380 \mathrm{~V} \text { (triângulo), } \\
100 \mathrm{cv} \text {. }\end{array}$ \\
\hline TE & $\begin{array}{l}1 \text { transformador elétrico } \\
\text { trifásico classe } 15 \mathrm{kV} \text {, } \\
150.000 \mathrm{VA} \text {. }\end{array}$ & $\begin{array}{l}1 \text { transformador elétrico } \\
\text { trifásico classe } 15 \mathrm{kV} \text {, } \\
91.000 \mathrm{VA} \text {. }\end{array}$ & $\begin{array}{l}1 \text { transformador elétrico } \\
\text { trifásico classe } 15 \mathrm{kV} \text {, } \\
93.200 \text { VA. }\end{array}$ & $\begin{array}{l}1 \text { transformador elétrico } \\
\text { trifásico classe } 15 \mathrm{kV} \text {, } \\
112.500 \text { VA. }\end{array}$ \\
\hline $\mathrm{AE}$ & $\begin{array}{l}\text { acessórios elétricos: } \\
\text { isoladores; eletrodutos; } \\
\text { conectores; hastes de } \\
\text { aterramento; cabos; etc. }\end{array}$ & $\begin{array}{|lr|}\text { acessórios } & \text { elétricos: } \\
\text { isoladores; } & \text { eletrodutos; } \\
\text { conectores; hastes de } \\
\text { aterramento; cabos; etc. } \\
\end{array}$ & $\begin{array}{|lr|}\text { acessórios } & \text { elétricos: } \\
\text { isoladores; } & \text { eletrodutos; } \\
\text { conectores; } & \text { hastes de } \\
\text { aterramento; } & \text { cabos; etc. } \\
\end{array}$ & $\begin{array}{l}\text { acessórios elétricos: } \\
\text { isoladores; eletrodutos; } \\
\text { conectores; hastes de } \\
\text { aterramento; cabos; etc. }\end{array}$ \\
\hline LAT & $\begin{array}{l}1 \text { linha de alta tensão de } \\
1800 \mathrm{~m} \text {, padrão CPFL. }\end{array}$ & $\begin{array}{l}1 \text { linha de alta tensão de } \\
1800 \mathrm{~m} \text {, padrão CPFL. } \\
\end{array}$ & $\begin{array}{l}1 \text { linha de alta tensão de } \\
1800 \mathrm{~m} \text {, padrão CPFL. }\end{array}$ & $\begin{array}{l}1 \text { linha de alta tensão de } \\
1800 \mathrm{~m} \text {, padrão CPFL. } \\
\end{array}$ \\
\hline $\mathrm{OCC}$ & $\begin{array}{l}1 \text { casa de bombas de } \\
14,00 \mathrm{~m}^{2} \text {, acabamento } \\
\text { rústico. }\end{array}$ & $\begin{array}{l}1 \text { casa de bombas de } \\
14,00 \mathrm{~m}^{2}, \text { acabamento } \\
\text { nústico. }\end{array}$ & $\begin{array}{l}1 \text { casa de bombas de } \\
14,00 \mathrm{~m}^{2}, \text { acabamento } \\
\text { nústico. }\end{array}$ & $\begin{array}{l}1 \text { casa de bombas de } \\
14,00 \mathrm{~m}^{2} \text {, acabamento } \\
\text { rústico. }\end{array}$ \\
\hline
\end{tabular}


APENDICE 8. Custos e composição do sistema à eletricidade na tarifa verde irrigante (EVI) para os tipos Comercial/Fabricante; Teórico/Econômico; Comercial/Teórico/Econômico; e Comercial/Econômico.

\section{Custos}

1.1. Custos de aquisição por item do sistema (US\$):

\begin{tabular}{|c|c|c|c|c|}
\hline Item do & \multicolumn{5}{|c|}{ Custo de Aquisição } \\
\cline { 2 - 5 } Sistema & Com./Fabr. & Teór./Econ. & Com./Teór./Econ. & Com./Econ. \\
\hline Tubulação de sucção & $\mathbf{9 2 7 , 0 0}$ & $\mathbf{8 5 8 , 2 8}$ & $\mathbf{8 5 8 , 2 8}$ & $\mathbf{9 2 7 , 0 0}$ \\
Tubulação de recalque & $14.549,44$ & $17.709,73$ & $16.336,46$ & $15.899,44$ \\
Bomba hidráulica & $10.126,64$ & $9.473,29$ & $9.551,40$ & $10.126,64$ \\
Motor elétrico & $4.580,68$ & $3.140,00$ & $3.196,33$ & $3.091,97$ \\
Banco de capacitores & 246,83 & 184.53 & 186,97 & 273,62 \\
Chave de partida & $6.294,46$ & $5.584,48$ & $5.657,29$ & $4.946,30$ \\
Transformador elétrico & $3.236,00$ & $2.489,71$ & $2.521,54$ & $2.800,00$ \\
Acessórios elétricos & $1.456,20$ & $1.120,37$ & $1.134,69$ & $1.260,00$ \\
Linha de alta tensão & $13.680,00$ & $13.680,00$ & $13.680,00$ & $13.680,00$ \\
Obras de constr. civil & $\mathbf{4 . 5 2 6 , 3 4}$ & 4.526 .34 & $4.526,34$ & $4.526,34$ \\
\hline Total & $\mathbf{5 9 . 6 2 3 , 5 9}$ & $\mathbf{5 8 . 7 6 6 , 7 3}$ & $\mathbf{5 7 . 6 4 9 , 3 1}$ & $\mathbf{5 7 . 5 3 1 , 3 1}$ \\
\hline
\end{tabular}

1.2. Custos fixos anual do sistema (US\$):

- Com./Fabr $6.108,09$

- Teór./Econ. $6.020,31$

- Com./Teór./Econ. $5.905,84$

- Com./Econ. $5.893,75$

1.3. Custos anual de bombeamento do sistema (US\$):

\begin{tabular}{|c|c|c|c|c|}
\hline Faturamento & Com./Fabr. & Teór./Econ. & Com./Teór./Econ. & Com./Econ. \\
\hline FD & $1.610,38$ & $1.465,92$ & $1.494,30$ & $1.444,51$ \\
FCsp & $1.395,11$ & $1.269,96$ & $1.294,55$ & $1.251,41$ \\
FCsfp & $2.726,06$ & $2.481,52$ & $2.526,57$ & $2.442,37$ \\
FCshe & 369,34 & 336,21 & 342,72 & 331,30 \\
FCup & 457,41 & 416,38 & 424,44 & 410,30 \\
FCufp & 803,36 & 731,29 & 745,45 & 720,61 \\
FCuhe & 108,84 & 99,08 & 101,00 & 97,63 \\
\hline Total & $\mathbf{7 . 4 7 0 , 5 1}$ & $\mathbf{6 . 8 0 0 , 3 6}$ & $\mathbf{6 . 9 3 2 , 0 2}$ & $\mathbf{6 . 6 9 8 , 1 3}$ \\
\hline
\end{tabular}

1.4. Custos anual com manutenção e reparos do sistema (US\$):

- Com./Fabr. 892,68

- Teór./Econ. 805,04

- Com./Teór./Econ. 802,63

- Com./Econ. 853,33 


\subsection{Custos anual total do sistema (US\$):}

- Com./Fabr.

$14.471,28$

- Teór./Econ.

$13.625,71$

- Com./Teór./Econ.

$13.640,49$

- Com./Econ.

$13.445,21$

\section{Composição do sistema}

\begin{tabular}{|c|c|c|c|c|}
\hline $\begin{array}{l}\text { Item } \\
\text { sistema }\end{array}$ & Com./Fabr. & Teár./Econ. & Com.Teór $/$ Lcon. & Com/Econ. \\
\hline $\mathrm{Ts}$ & $\begin{array}{l}1 \text { válv. sucção } 300 \mathrm{~mm} \text {; } \\
1 \text { curva } 90^{\circ} 300 \mathrm{~mm} ; 1 \\
\text { luva cônica excêntrica } \\
300 \text { × } 200 \mathrm{~mm} ; 2 \text { tubos } \\
300 \mathrm{~mm} \text {. }\end{array}$ & $\begin{array}{l}1 \text { válv. sucção } 300 \mathrm{~mm} \text {; } \\
1 \text { curva } 90^{\circ} 300 \mathrm{~mm} ; 1 \\
\text { luva cônica excêntrica } \\
300 \mathrm{~mm} ; 2 \text { tubos } 300 \\
\mathrm{~mm} \text {. }\end{array}$ & $\begin{array}{l}1 \text { válv, sucção } 300 \mathrm{~mm} \text {; } \\
1 \text { curva } 90^{\circ} 300 \mathrm{~mm} ; 1 \\
\text { luva cônica excêntrica } \\
300 \mathrm{~mm} ; 2 \text { tubos } 300 \\
\mathrm{~mm} \text {. }\end{array}$ & $\begin{array}{l}1 \text { válv. sucção } 300 \mathrm{~mm} ; \\
1 \text { curva } 90^{\circ} 300 \mathrm{~mm} ; 1 \\
\text { luva cônica excêntrica } \\
300 \times 200 \mathrm{~mm} ; 2 \text { tubos } \\
300 \mathrm{~mm} .\end{array}$ \\
\hline $\mathrm{Tr}$ & $\begin{array}{l}1 \text { luva cônica conc. } 250 \\
\text { X } 150 \mathrm{~mm} ; 1 \text { válvula } \\
\text { retenção } 250 \mathrm{~mm} ; 1 \\
\text { válvula gaveta } 250 \mathrm{~mm} ; \\
2 \text { curvas } 60^{\circ} 250 \mathrm{~mm} ; 1 \\
\text { curva } 90^{\circ} 250 \mathrm{~mm} ; 1 \\
\text { curva } 90^{\circ} 200 \mathrm{~mm} ; 42 \\
\text { tubos } 250 \mathrm{~mm} ; 50 \text { tubos } \\
200 \mathrm{~mm} .\end{array}$ & $\begin{array}{l}1 \text { luva cônica conc. } 265 \\
\text { mm; } 1 \text { válvula retenção } \\
265 \mathrm{~mm} ; 1 \text { válv. gaveta } \\
265 \mathrm{~mm} ; 2 \text { curva } 60^{\circ} \\
265 \mathrm{~mm} ; 2 \text { curvas } 90^{\circ} \\
265 \mathrm{~mm} ; 92 \text { tubos } 265 \\
\mathrm{~mm} .\end{array}$ & $\begin{array}{l}1 \text { luva cônica conc. } 250 \\
\text { mm; 1 válvula retenção } \\
250 \mathrm{~mm} ; 1 \text { válv. gaveta } \\
250 \mathrm{~mm} ; 2 \text { curva } 60^{\circ} \\
250 \mathrm{~mm} ; 2 \text { curvas } 90^{\circ} \\
250 \mathrm{~mm} ; 92 \text { tubos } 250 \\
\mathrm{~mm} .\end{array}$ & $\begin{array}{l}1 \text { luva cônica conc. } 250 \\
\text { x } 150 \mathrm{~mm} ; 1 \text { válvula } \\
\text { retenção } 250 \mathrm{~mm} ; 1 \\
\text { válv. gaveta } 250 \mathrm{~mm} ; 2 \\
\text { curvas } 60^{\circ} 250 \mathrm{~mm} ; 2 \\
\text { curvas } 90^{\circ} 250 \mathrm{~mm} ; 92 \\
\text { tubos } 250 \mathrm{~mm} \text {. }\end{array}$ \\
\hline $\mathrm{BH}$ & $\begin{array}{l}1 \text { bomba multiestágio, } \\
76,7 \mathrm{cv} \text {, } 125 / 2 \text {, rotor } \\
320 \mathrm{~mm} \text {, sucção } 200 \\
\mathrm{~mm} \text {, recalque } 150 \mathrm{~mm} \text {, } \\
\text { rendimento } 78 \% \text { (real). }\end{array}$ & $\begin{array}{l}1 \text { bomba multiestágio, } \\
67,5 \mathrm{cv} \text {, rendimento } \\
78 \% \text { (modelo). }\end{array}$ & $\begin{array}{l}1 \text { bomba multiestágio, } \\
68,8 \quad \mathrm{cv} \text {, rendimento } \\
78 \% \text { (modelo). }\end{array}$ & $\begin{array}{l}1 \text { bomba multiestágio, } \\
68,8 \mathrm{cv}, 125 / 2 \text {, rotor } \\
320 \mathrm{~mm} \text {, sucção } 200 \\
\mathrm{~mm} \text {, recalque } 150 \mathrm{~mm} \text {, } \\
\text { rendimento } 78 \% \text { (real). }\end{array}$ \\
\hline $\mathrm{ME}$ & $\begin{array}{l}1 \text { motor trifásico } 60 \mathrm{~Hz} \text {, } \\
1800 \mathrm{rpm}, 380 \mathrm{~V} \text {, classe } \\
\text { " } \mathrm{B} \text { ", } 125 \mathrm{cv} \text {, rendimen- } \\
\text { to } 91 \% \text { (real), cos } \varphi 0,87 \\
\text { (real). }\end{array}$ & $\begin{array}{l}1 \text { motor trifásico } 60 \mathrm{~Hz} \text {, } \\
1800 \mathrm{rpm}, 380 \mathrm{~V} \text {, classe } \\
\text { "B", } 95,2 \mathrm{cv} \text {, rendimen- } \\
\text { to } 88 \% \text { (modelo), cos } \varphi \\
0,87 \text { (modelo). }\end{array}$ & $\begin{array}{l}1 \text { motor trifásico } 60 \mathrm{~Hz} \\
1800 \mathrm{rpm}, 380 \mathrm{~V} \text {, classe } \\
\text { " } \mathrm{B} \text { ", } 97,1 \mathrm{cv} \text {, rendimen- } \\
\text { to } 88 \% \text { (modelo), cos } \varphi \\
0,87 \text { (modelo). }\end{array}$ & $\begin{array}{l}1 \text { motor trifásico } 60 \mathrm{~Hz} \\
1800 \mathrm{rpm}, 220 / 380 \mathrm{~V} \text {, } \\
\text { classe "B", } 100 \mathrm{cv} \text {, } \\
\text { rendimento } 91 \% \text { (real), } \\
\cos \varphi 0,85 \text { (real). }\end{array}$ \\
\hline $\mathrm{BC}$ & $\begin{array}{l}1 \text { banco capac. trifásico } \\
380 \mathrm{~V}, 17.500 \mathrm{VAr} \text {. }\end{array}$ & $\begin{array}{l}1 \text { banco capac. trifásico } \\
380 \mathrm{~V}, 9.900 \mathrm{VAr} \text {. }\end{array}$ & $\begin{array}{l}1 \text { banco capac. trifásico } \\
380 \mathrm{~V}, 10.000 \mathrm{VAr} .\end{array}$ & $\begin{array}{l}1 \text { banco capac. trifásico } \\
380 \mathrm{~V}, 20.000 \mathrm{VAr} \text {. }\end{array}$ \\
\hline $\mathrm{CP}$ & $\begin{array}{l}1 \text { ch. partida compensa- } \\
\text { dora } 380 \mathrm{~V} \text { (triângulo), } \\
125 \mathrm{cv} \text {. }\end{array}$ & $\begin{array}{l}1 \mathrm{ch} \text {. partida compensa- } \\
\text { dora } 380 \mathrm{~V} \text { (triângulo), } \\
95,2 \mathrm{cv} \text {. }\end{array}$ & $\begin{array}{l}1 \mathrm{ch} \text {. partida compensa- } \\
\text { dora } 380 \mathrm{~V} \text { (triângulo), } \\
97,1 \mathrm{cv} \text {. }\end{array}$ & $\begin{array}{l}1 \text { ch. partida compensa- } \\
\text { dora } 380 \mathrm{~V} \text { (triângulo), } \\
100 \mathrm{cv} \text {. }\end{array}$ \\
\hline TE & $\begin{array}{l}1 \text { transformador elétrico } \\
\text { trifásico classe } 15 \mathrm{kV} \text {, } \\
150.000 \mathrm{VA} \text {. }\end{array}$ & $\begin{array}{l}1 \text { transformador elétrico } \\
\text { trifásico classe } 15 \mathrm{kV} \text {, } \\
91.400 \mathrm{VA} \text {. }\end{array}$ & $\begin{array}{l}1 \text { transformador elétrico } \\
\text { trifásico classe } 15 \mathrm{kV} \text {, } \\
93.200 \mathrm{VA} \text {. }\end{array}$ & $\begin{array}{l}1 \text { transformador elétrico } \\
\text { trifásico classe } 15 \mathrm{kV} \text {, } \\
112.500 \mathrm{VA} \text {. }\end{array}$ \\
\hline$\overline{\mathrm{AE}}$ & $\begin{array}{lr}\text { acessórios } & \text { elétricos: } \\
\text { isoladores; } & \text { eletrodutos; } \\
\text { conectores; hastes de } \\
\text { aterramento; cabos; etc. }\end{array}$ & 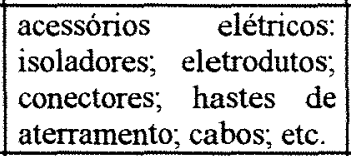 & $\begin{array}{lr}\text { acessórios } & \text { elétricos: } \\
\text { isoladores; } & \text { eletrodutos; } \\
\text { conectores; hastes de } \\
\text { aterramento; cabos; etc. }\end{array}$ & $\begin{array}{l}\text { acessórios elétricos: } \\
\text { isoladores; eletrodutos; } \\
\text { conectores; hastes de } \\
\text { aterramento; cabos; etc. }\end{array}$ \\
\hline LAT & $\begin{array}{l}1 \text { linha de alta tensão de } \\
1800 \mathrm{~m} \text {, padrão CPFL. }\end{array}$ & $\begin{array}{l}1 \text { linha de alta tensão de } \\
1800 \mathrm{~m} \text {, padrão CPFL. }\end{array}$ & $\begin{array}{l}1 \text { linha de alta tensão de } \\
1800 \mathrm{~m} \text {, padrão CPFL. }\end{array}$ & $\begin{array}{l}1 \text { linha de alta tensão de } \\
1800 \mathrm{~m} \text {, padrão CPFL. }\end{array}$ \\
\hline $\mathrm{OCC}$ & $\begin{array}{l}1 \text { casa de bombas de } \\
14,00 \mathrm{~m}^{2}, \text { acabamento } \\
\text { nústico. }\end{array}$ & $\begin{array}{l}1 \text { casa de bombas de } \\
14,00 \mathrm{~m}^{2}, \text { acabamento } \\
\text { nústico. }\end{array}$ & $\begin{array}{l}1 \text { casa de bombas de } \\
14,00 \mathrm{~m}^{2}, \text { acabamento } \\
\text { nústico. }\end{array}$ & $\begin{array}{l}1 \text { casa de bombas de } \\
14,00 \mathrm{~m}^{2} \text {, acabamento } \\
\text { rústico. }\end{array}$ \\
\hline
\end{tabular}


APÊNDICE 9. Custos e composição do sistema à eletricidade na tarifa azul normal (EAN) para os tipos Comercial/Fabricante; Teórico/Econômico; Comercial/Teórico/Econômico; e Comercial/Econômico.

\section{Custos}

1.1. Custos de aquisição por item do sistema (US\$):

\begin{tabular}{|c|c|c|c|c|}
\hline Item do & \multicolumn{4}{|c|}{ Custo de Aquisição } \\
\cline { 2 - 5 } Sistema & Com./Fabr. & Teór./Econ. & Com./Teór./Econ. & Com./Econ. \\
\hline Tubulação de sucção & $\mathbf{9 2 7 , 0 0}$ & $\mathbf{8 5 8 , 2 8}$ & $\mathbf{8 5 8 , 2 8}$ & 927,00 \\
Tubulação de recalque & $14.549,44$ & $19.614,78$ & $20.869,17$ & $20.482,74$ \\
Bomba hidráulica & $10.126,64$ & $9.401,20$ & $9.368,49$ & $10.126,64$ \\
Motor elétrico & $4.580,68$ & $3.088,49$ & $3.065,26$ & $3.091,97$ \\
Banco de capacitores & 246,83 & 182,29 & 181,27 & 273,62 \\
Chave de partida & $6.294,46$ & $5.517,58$ & $5.487,32$ & $4.946,30$ \\
Transformador elétrico & $3.236,00$ & $2.460,46$ & $2.447,23$ & $2.800,00$ \\
Acessórios elétricos & $1.456,20$ & $1.107,21$ & $1.101,25$ & $1.260,00$ \\
Linha de alta tensão & $13.680,00$ & $13.680,00$ & $13.680,00$ & $13.680,00$ \\
Obras de constr. civil & $4.526,34$ & $\mathbf{4 . 5 2 6 , 3 4}$ & $4.526,34$ & $4.526,34$ \\
\hline Total & $\mathbf{5 9 . 6 2 3 , 5 9}$ & $\mathbf{6 0 . 4 3 6 , 6 3}$ & $\mathbf{6 1 . 5 8 4 , 6 3}$ & $\mathbf{6 2 . 1 1 4 , 6 1}$ \\
\hline
\end{tabular}

1.2. Custos fixos anual do sistema (US\$):

- Com./Fabr. $6.108,09$

- Teór./Econ. $6.191,38$

- Com./Teór./Econ. $6.308,99$

- Com./Econ. $6.363,28$

1.3. Custos anual de bombeamento do sistema (US\$):

\begin{tabular}{|c|c|c|c|c|}
\hline Faturamento & Com./Fabr. & Teór./Econ. & Com./Teór./Econ. & Com./Econ. \\
\hline FDp & $6.145,07$ & $5.492,93$ & $5.448,40$ & $5.268,78$ \\
FDfp & $2.050,32$ & $1.832,73$ & $1.817,87$ & $1.757,94$ \\
FCsp & 308,39 & 275,66 & 273,42 & 274,39 \\
FCsfp & $3.958,63$ & $3.538,52$ & $3.509,84$ & $3.394,13$ \\
FCup & 95,15 & 85,05 & 84,36 & $\mathbf{8 1 , 5 8}$ \\
FCufp & $1.166,58$ & $1.042,78$ & $1.034,33$ & 1000,23 \\
\hline Total & $\mathbf{1 3 . 7 2 4 , 1 4}$ & $\mathbf{1 2 . 2 6 7 , 6 7}$ & $\mathbf{1 2 . 1 6 8 , 2 2}$ & $\mathbf{1 1 . 7 6 7 , 0 7}$ \\
\hline
\end{tabular}

1.4. Custos anual com manutenção e reparos do sistema (US\$):

- Com./Fabr. 892,68

- Teór./Econ. 811,10

- Com./Teór./Econ. 816,20

- Com./Econ. $\mathbf{8 8 0 , 8 3}$ 


\subsection{Custos anual total do sistema (US\$):}

• Com./Fabr. $\ldots \ldots \ldots \ldots \ldots \ldots \ldots . . .20 .724,91$
- Teór./Econ....................... 19.270,16
- Com./Teór./Econ. .......... 19.293,41
- Com./Econ..................... 19.011,18

\section{Composição do sistema}

\begin{tabular}{|c|c|c|c|c|}
\hline $\begin{array}{l}\text { Item } \\
\text { sistema }\end{array}$ & Com. Fabr. & Teór/Econ. & Com.lTeór./Econ. & Com./Econ. \\
\hline $\mathrm{Ts}$ & $\begin{array}{l}1 \text { válv. sucção } 300 \mathrm{~mm} \text {; } \\
1 \text { curva } 90^{\circ} 300 \mathrm{~mm} ; 1 \\
\text { luva cônica excêntrica } \\
300 \times 200 \mathrm{~mm} ; 2 \text { tubos } \\
300 \mathrm{~mm} \text {. }\end{array}$ & $\begin{array}{l}1 \text { válv. sucção } 300 \mathrm{~mm} \text {; } \\
1 \text { curva } 90^{\circ} 300 \mathrm{~mm} ; 1 \\
\text { luva cônica excêntrica } \\
300 \mathrm{~mm} ; 2 \text { tubos } 300 \\
\mathrm{~mm} \text {. }\end{array}$ & $\begin{array}{l}1 \text { válv. sucção } 300 \mathrm{~mm} \text {; } \\
1 \text { curva } 90^{\circ} 300 \mathrm{~mm} ; 1 \\
\text { luva cônica excêntrica } \\
300 \mathrm{~mm} ; 2 \text { tubos } 300 \\
\mathrm{~mm} \text {. }\end{array}$ & $\begin{array}{l}1 \text { válv. sucção } 300 \mathrm{~mm} \text {; } \\
1 \text { curva } 90^{\circ} 300 \mathrm{~mm} ; 1 \\
\text { luva cônica excêntrica } \\
300 \times 200 \mathrm{~mm} ; 2 \text { tubos } \\
300 \mathrm{~mm} \text {. }\end{array}$ \\
\hline $\mathrm{Tr}$ & $\begin{array}{l}1 \text { luva cônica conc. } 250 \\
\text { x } 150 \mathrm{~mm} ; 1 \text { válvula } \\
\text { retenção } 250 \mathrm{~mm}, 1 \\
\text { válvula gaveta } 250 \mathrm{~mm} ; \\
2 \text { curvas } 60^{\circ} 250 \mathrm{~mm} ; 1 \\
\text { curva } 90^{\circ} 250 \mathrm{~mm} ; 1 \\
\text { curva } 90^{\circ} 200 \mathrm{~mm} ; 42 \\
\text { tubos } 250 \mathrm{~mm} ; 50 \text { tubos } \\
200 \mathrm{~mm} .\end{array}$ & $\begin{array}{l}1 \text { luva cônica conc. } 286 \\
\text { mm; 1 válvula retenção } \\
286 \mathrm{~mm} ; 1 \text { válv. gaveta } \\
286 \mathrm{~mm} ; 2 \text { curva } 60^{\circ} \\
286 \mathrm{~mm} ; 2 \text { curvas } 90^{\circ} \\
286 \mathrm{~mm} ; 92 \text { tubos } 286 \\
\mathrm{~mm} .\end{array}$ & $\begin{array}{l}1 \text { luva cônica conc. } 300 \\
\text { mm; 1 válvula retenção } \\
300 \mathrm{~mm} ; 1 \text { válv. gaveta } \\
300 \mathrm{~mm} ; 2 \text { curva } 60^{\circ} \\
300 \mathrm{~mm} ; 2 \text { curvas } 90^{\circ} \\
300 \mathrm{~mm} ; 92 \text { tubos } 300 \\
\mathrm{~mm} .\end{array}$ & $\begin{array}{l}1 \text { luva cônica conc. } 300 \\
\times 150 \mathrm{~mm} ; 1 \text { válvula } \\
\text { retenção } 300 \mathrm{~mm} ; 1 \\
\text { válv. gaveta } 300 \mathrm{~mm} ; 2 \\
\text { curvas } 60^{\circ} 300 \mathrm{~mm} ; 2 \\
\text { curvas } 90^{\circ} 300 \mathrm{~mm} ; 92 \\
\text { tubos } 300 \mathrm{~mm} \text {. }\end{array}$ \\
\hline $\mathrm{BH}$ & $\begin{array}{l}1 \text { bomba multiestágio, } \\
76,7 \mathrm{cv} \text {, } 125 / 2 \text {, rotor } \\
320 \mathrm{~mm} \text {, sucção } 200 \\
\text { mm, recalque } 150 \mathrm{~mm} \text {, } \\
\text { rendimento } 78 \% \text { (real). }\end{array}$ & $\begin{array}{l}1 \text { bomba multiestágio, } \\
66,3 \mathrm{cv} \text {, rendimento } \\
78 \% \text { (modelo). }\end{array}$ & $\begin{array}{l}1 \text { bomba multiestagio, } \\
65,8 \quad \mathrm{cv} \text {, rendimento } \\
78 \% \text { (modelo). }\end{array}$ & $\begin{array}{l}1 \text { bomba multiestágio, } \\
65,8 \mathrm{cv}, 125 / 2 \text {, rotor } \\
295 \mathrm{~mm} \text {, sucção } 200 \\
\text { mm, recalque } 150 \mathrm{~mm} \text {, } \\
\text { rendimento } 78 \% \text { (real). }\end{array}$ \\
\hline $\mathrm{ME}$ & $\begin{array}{l}1 \text { motor trifásico } 60 \mathrm{~Hz} \text {, } \\
1800 \mathrm{rpm}, 380 \mathrm{~V} \text {, classe } \\
\text { "B", } 125 \mathrm{cv} \text {, rendimen- } \\
\text { to } 91 \% \text { (real), cos } \varphi 0,87 \\
\text { (real). }\end{array}$ & $\begin{array}{l}1 \text { motor trifásico } 60 \mathrm{~Hz} \text {, } \\
1800 \mathrm{rpm}, 380 \mathrm{~V} \text {, classe } \\
\text { " } \mathrm{B} ", 93,6 \mathrm{cv} \text {, rendimen- } \\
\text { to } 88 \% \text { (modelo), cos } \varphi \\
0,87 \text { (modelo). }\end{array}$ & $\begin{array}{l}1 \text { motor trifásico } 60 \mathrm{~Hz} \text {, } \\
1800 \mathrm{rpm}, 380 \mathrm{~V} \text {, classe } \\
\text { "c } \mathrm{B} \text { ", } 92,8 \mathrm{cv} \text {, rendimen- } \\
\text { to } 88 \% \text { (modelo), cos } \varphi \\
0,87 \text { (modelo). }\end{array}$ & $\begin{array}{l}1 \text { motor trifásico } 60 \mathrm{~Hz} \text {, } \\
1800 \mathrm{rpm}, 220 / 380 \mathrm{~V} \text {, } \\
\text { classe "B", } 100 \mathrm{cv} \text {, } \\
\text { rendimento } 91 \% \text { (real), } \\
\cos \varphi 0,85 \text { (real).. }\end{array}$ \\
\hline $\mathrm{BC}$ & $\begin{array}{l}1 \text { banco capac. trifásico } \\
380 \mathrm{~V}, 17.500 \mathrm{VAr} \text {. }\end{array}$ & $\begin{array}{l}1 \text { banco capac. trifásico } \\
380 \mathrm{~V}, 9.700 \mathrm{VAr} \text {. }\end{array}$ & $\begin{array}{l}1 \text { banco capac. trifásico } \\
380 \mathrm{~V}, 9.600 \mathrm{VAr} \text {. }\end{array}$ & $\begin{array}{l}1 \text { banco capac. trifásico } \\
380 \mathrm{~V}, 20.000 \mathrm{VAr} \text {. }\end{array}$ \\
\hline $\mathrm{CP}$ & $\begin{array}{l}1 \mathrm{ch} \text {. partida compensa- } \\
\text { dora } 380 \mathrm{~V} \text { (triângulo), } \\
125 \mathrm{cv} \text {. }\end{array}$ & $\begin{array}{l}1 \mathrm{ch} \text {. partida compensa- } \\
\text { dora } 380 \mathrm{~V} \text { (triângulo), } \\
93,6 \mathrm{cv} \text {. }\end{array}$ & $\begin{array}{l}1 \mathrm{ch} \text {. partida compensa- } \\
\text { dora } 380 \mathrm{~V} \text { (triângulo), } \\
92,8 \mathrm{cv} \text {. }\end{array}$ & $\begin{array}{l}1 \text { ch. partida compensa- } \\
\text { dora } 380 \mathrm{~V} \text { (triângulo), } \\
100 \mathrm{cv} .\end{array}$ \\
\hline $\mathrm{TE}$ & $\begin{array}{l}1 \text { transformador elétrico } \\
\text { trifásico classe } 15 \mathrm{kV} \text {, } \\
150.000 \mathrm{VA} \text {. }\end{array}$ & $\begin{array}{l}1 \text { transformador elétrico } \\
\text { trifásico classe } 15 \mathrm{kV} \text {, } \\
89.800 \text { VA. }\end{array}$ & $\begin{array}{l}1 \text { transformador elétrico } \\
\text { trifásico classe } 15 \mathrm{kV} \text {, } \\
89.100 \text { VA. }\end{array}$ & $\begin{array}{l}1 \text { transformador elétrico } \\
\text { trifásico classe } 15 \mathrm{kV} \text {, } \\
112.500 \mathrm{VA} \text {. }\end{array}$ \\
\hline $\mathrm{AE}$ & 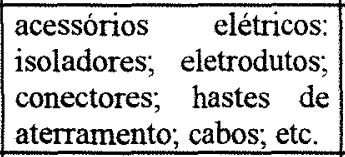 & $\begin{array}{l}\text { acessórios } \text { elétricos: } \\
\text { isoladores; eletrodutos; } \\
\text { conectores; hastes de } \\
\text { aterramento; cabos; etc. }\end{array}$ & $\begin{array}{|lr|}\text { acessórios } & \text { elétricos: } \\
\text { isoladores; } & \text { eletrodutos; } \\
\text { conectores; } & \text { hastes de } \\
\text { aterramento; cabos; etc. }\end{array}$ & 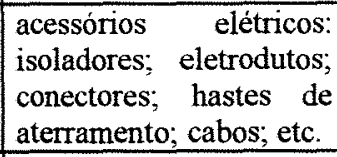 \\
\hline LAT & $\begin{array}{l}1 \text { linha de alta tensão de } \\
1800 \mathrm{~m} \text {, padrão CPFL. }\end{array}$ & $\begin{array}{l}1 \text { linha de alta tensão de } \\
1800 \mathrm{~m} \text {, padrão } \mathrm{CPFL} \text {. }\end{array}$ & $\begin{array}{l}1 \text { linha de alta tensão de } \\
1800 \mathrm{~m} \text {, padrão CPFL. }\end{array}$ & $\begin{array}{l}1 \text { linha de alta tensão de } \\
1800 \mathrm{~m} \text {, padrão CPFL. }\end{array}$ \\
\hline $\mathrm{OCC}$ & $\begin{array}{l}1 \text { casa de bombas de } \\
14,00 \mathrm{~m}^{2}, \text { acabamento } \\
\text { rústico. }\end{array}$ & $\begin{array}{l}1 \text { casa de bombas de } \\
14,00 \mathrm{~m}^{2}, \text { acabamento } \\
\text { rústico. }\end{array}$ & $\begin{array}{l}1 \text { casa de bombas de } \\
14,00 \mathrm{~m}^{2}, \text { acabamento } \\
\text { rústico. }\end{array}$ & $\begin{array}{l}1 \text { casa de bombas de } \\
14,00 \mathrm{~m}^{2}, \text { acabamento } \\
\text { nustico. }\end{array}$ \\
\hline
\end{tabular}


APÊNDICE 10. Custos e composição do sistema à eletricidade na tarifa azul irrigante (EAI) para os tipos Comercial/Fabricante; Teórico/Econômico; Comercial/Teórico/Econômico; e Comercial/Econômico.

\section{Custos}

1.1. Custos de aquisição por item do sistema (US\$):

\begin{tabular}{|c|c|c|c|c|}
\hline \multirow{2}{*}{ Item do } & \multicolumn{4}{|c|}{ Custo de Aquisição } \\
\cline { 2 - 5 } Sistema & Com./Fabr. & Teór./Econ. & Com./Teór./Econ. & Com./Econ. \\
\hline Tubulação de sucção & 927,00 & 858,28 & 858,28 & 927,00 \\
Tubulação de recalque & $14.549,44$ & $19.299,18$ & $20.869,17$ & $20.482,74$ \\
Bomba hidráulica & $10.126,64$ & $9.411,00$ & $9.368,49$ & $10.126,64$ \\
Motor elétrico & $4.580,68$ & $3.095,46$ & $3.065,26$ & $3.091,97$ \\
Banco de capacitores & 246,83 & 182,59 & 181,27 & 273,62 \\
Chave de partida & $6.294,46$ & $5.526,65$ & $5.487,32$ & $4.946,30$ \\
Transformador elétrico & $3.236,00$ & $2.464,43$ & $2.447,23$ & $2.800,00$ \\
Acessórios elétricos & $1.456,20$ & $1.108,99$ & $1.101,25$ & $1.260,00$ \\
Linha de alta tensão & $13.680,00$ & $13.680,00$ & $13.680,00$ & $13.680,00$ \\
Obras de constr. civil & $\mathbf{4 . 5 2 6 , 3 4}$ & $4.526,34$ & $4.526,34$ & $4.526,34$ \\
\hline Total & $\mathbf{5 9 . 6 2 3 , 5 9}$ & $\mathbf{6 0 . 1 5 2 , 9 3}$ & $\mathbf{6 1 . 5 8 4 , 6 3}$ & $\mathbf{6 2 . 1 1 4 , 6 1}$ \\
\hline
\end{tabular}

1.2. Custos fixos anual do sistema (US\$):
- Com./Fabr.
$6.108,09$
- Teór./Econ.
$6.162,32$
- Com./Teór./Econ.
$6.308,99$
- Com./Econ.
$6.363,28$

1.3. Custos anual de bombeamento do sistema (US\$):

\begin{tabular}{|c|c|c|c|c|}
\hline Faturamento & Com./Fabr. & Teór./Econ. & Com./Teór./Econ. & Com./Econ. \\
\hline FDp & $6.145,07$ & $5.506,31$ & $5.448,40$ & $5.268,78$ \\
FDfp & $2.050,32$ & $1.837,19$ & $1.817,87$ & $1.757,94$ \\
FCsp & 308,39 & 276,33 & 273,42 & 274,39 \\
FCsfp & $2.668,41$ & $2.391,04$ & $2.365,89$ & $2.287,89$ \\
FCshe & 369,48 & 331,07 & 327,58 & 316,78 \\
FCup & 95,15 & 85,26 & 84,36 & 81,58 \\
FCufp & 786,37 & 704,63 & 697,22 & 674,23 \\
FCuhe & 108,88 & 97,56 & 96,54 & 93,36 \\
\hline Total & $\mathbf{1 2 . 5 3 2 , 0 5}$ & $\mathbf{1 1 . 2 2 9 , 3 8}$ & $\mathbf{1 1 . 1 1 1 , 2 8}$ & $\mathbf{1 0 . 7 4 4 , 9 7}$ \\
\hline
\end{tabular}

1.4. Custos anual com manutenção e reparos do sistema (US\$):

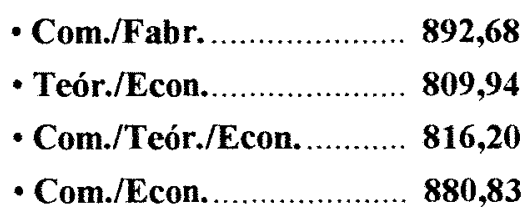




\subsection{Custos anual total do sistema (US\$):}

- Com./Fabr.

$19.532,82$

- Teór./Econ.

18.201,64

- Com./Teór./Econ.

$18.236,47$

- Com./Econ.

$17.989,08$

\section{Composição do sistema}

\begin{tabular}{|c|c|c|c|c|}
\hline $\begin{array}{l}\text { Item } \\
\text { sistema }\end{array}$ & Com./Tabr. & Teór/Rcon. & Com./Teór./Econ. & Com./Econ. \\
\hline Ts & $\begin{array}{l}1 \text { válv. sucção } 300 \mathrm{~mm} ; \\
1 \text { curva } 90^{\circ} 300 \mathrm{~mm} ; 1 \\
\text { luva cônica excêntrica } \\
300 \times 200 \mathrm{~mm} ; 2 \text { tubos } \\
300 \mathrm{~mm} \text {. }\end{array}$ & \begin{tabular}{|l|}
1 válv. sucção $300 \mathrm{~mm} ;$ \\
1 curva $90^{\circ} \quad 300 \mathrm{~mm} ; 1$ \\
luva cônica excêntrica \\
$300 \mathrm{~mm} ; 2$ tubos 300 \\
$\mathrm{~mm}$.
\end{tabular} & $\begin{array}{l}1 \text { válv. sucção } 300 \mathrm{~mm} ; \\
1 \text { curva } 90^{\circ} \quad 300 \mathrm{~mm} ; 1 \\
\text { luva cônica } \\
300 \mathrm{~mm} ; 2 \text { tubos } 300 \\
\mathrm{~mm} .\end{array}$ & $\begin{array}{l}1 \text { válv. sucção } 300 \mathrm{~mm} ; \\
1 \text { curva } 90^{\circ} 300 \mathrm{~mm} ; 1 \\
\text { luva cônica excêntrica } \\
300 \times 200 \mathrm{~mm} ; 2 \text { tubos } \\
300 \mathrm{~mm} \text {. }\end{array}$ \\
\hline $\operatorname{Tr}$ & $\begin{array}{l}1 \text { luva cônica conc. } 250 \\
\text { x } 150 \mathrm{~mm} ; 1 \mathrm{válvula} \\
\text { retenção } 250 \mathrm{~mm} ; 1 \\
\text { válvula gaveta } 250 \mathrm{~mm} ; \\
2 \text { curvas } 60^{\circ} 250 \mathrm{~mm} ; 1 \\
\text { curva } 90^{\circ} 250 \mathrm{~mm} ; 1 \\
\text { curva } 90^{\circ} 200 \mathrm{~mm} ; 42 \\
\text { tubos } 250 \mathrm{~mm} ; 50 \text { tubos } \\
200 \mathrm{~mm} \text {. }\end{array}$ & $\begin{array}{l}1 \text { luva cônica conc. } 283 \\
\text { mm; } 1 \text { válvula retenção } \\
283 \mathrm{~mm} ; 1 \text { válv. gaveta } \\
283 \mathrm{~mm} ; 2 \text { curva } 60^{\circ} \\
283 \mathrm{~mm} ; 2 \text { curvas } 90^{\circ} \\
283 \mathrm{~mm} ; 92 \text { tubos } 283 \\
\mathrm{~mm} .\end{array}$ & $\begin{array}{l}1 \text { luva cônica conc. } 300 \\
\mathrm{~mm} ; 1 \text { válvula retenção } \\
300 \mathrm{~mm} ; 1 \text { válv. gaveta } \\
300 \mathrm{~mm} ; 2 \text { curva } 60^{\circ} \\
300 \mathrm{~mm} ; 2 \text { curvas } 90^{\circ} \\
300 \mathrm{~mm} ; 92 \text { tubos } 300 \\
\mathrm{~mm} .\end{array}$ & $\begin{array}{l}1 \text { luva cônica conc. } 300 \\
\text { x } 150 \mathrm{~mm} ; 1 \mathrm{lálvula} \\
\text { retenção } 300 \mathrm{~mm} ; 1 \\
\text { válv. gaveta } 300 \mathrm{~mm} ; 2 \\
\text { curvas } 60^{\circ} 300 \mathrm{~mm} ; 2 \\
\text { curvas } 90^{\circ} 300 \mathrm{~mm} ; 92 \\
\text { tubos } 300 \mathrm{~mm} .\end{array}$ \\
\hline $\mathrm{BH}$ & 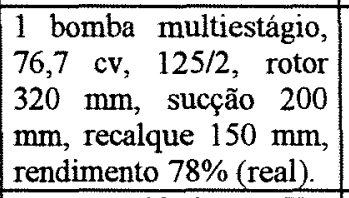 & $\begin{array}{l}1 \text { bomba multiestágio, } \\
66,5 \text { cv, rendimento } \\
78 \% \text { (modelo) }\end{array}$ & 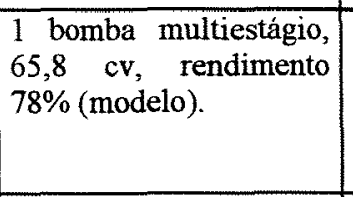 & $\begin{array}{l}1 \text { bomba multiestágio, } \\
65,8 \mathrm{cv}, 125 / 2 \text {, rotor } \\
295 \mathrm{~mm}, \text { sucção } 200 \\
\mathrm{~mm} \text {, recalque } 150 \mathrm{~mm} \text {, } \\
\text { rendimento } 78 \% \text { (real). }\end{array}$ \\
\hline $\mathrm{ME}$ & $\begin{array}{l}1 \text { motor trifásico } 60 \mathrm{~Hz} \\
1800 \mathrm{rpm}, 380 \mathrm{~V} \text {, classe } \\
\text { "B", } 125 \mathrm{cv}, \text { rendimen- } \\
\text { to } 91 \% \text { (real), cos } \varphi, 87 \\
\text { (real). }\end{array}$ & $\begin{array}{l}1 \text { motor trifäsico } 60 \mathrm{~Hz}, \\
1800 \mathrm{rpm}, 380 \mathrm{~V} \text {, classe } \\
\text { " } \mathrm{B} ", 93,8 \mathrm{cv} \text {, rendimen- } \\
\text { to } 88 \% \text { (modelo), cos } \varphi \\
0,87 \text { (modelo). } \\
\end{array}$ & $\begin{array}{l}1 \text { motor trifásico } 60 \mathrm{~Hz} \\
1800 \mathrm{rpm}, 380 \mathrm{~V} \text {, classe } \\
\text { " } \mathrm{B} \text { ", } 92,8 \mathrm{cv} \text {, rendimen- } \\
\text { to } 88 \% \text { (modelo), cos } \varphi \\
0,87 \text { (modelo). } \\
\end{array}$ & $\begin{array}{l}1 \text { motor trifásico } 60 \mathrm{~Hz} \text {, } \\
1800 \mathrm{pm}, 220 / 380 \mathrm{~V} \text {, } \\
\text { classe "B", } 100 \mathrm{cv} \text {, } \\
\text { rendimento } 91 \% \text { (real), } \\
\cos \varphi 0.85 \text { (real). }\end{array}$ \\
\hline $\mathrm{BC}$ & $\begin{array}{l}1 \text { banco capac. trifásico } \\
380 \mathrm{~V}, 17.500 \text { VAr. }\end{array}$ & $\begin{array}{l}\text { trifásico } \\
\text { VAr. }\end{array}$ & $\begin{array}{l}\text { trifásico } \\
\text { VAr. }\end{array}$ & $\begin{array}{l}1 \text { banco ce } \\
380 \mathrm{~V}, 20 \\
\end{array}$ \\
\hline $\mathrm{CP}$ & $\begin{array}{l}1 \text { ch. partida compensa- } \\
\text { dora } 380 \mathrm{~V} \text { (triângulo), } \\
125 \mathrm{cv} \text {. }\end{array}$ & $\begin{array}{l}1 \text { ch. pa } \\
\text { dora } 38 \\
93,8 \text { cv. } \\
\end{array}$ & $\begin{array}{l}\text { ensa- } \\
\text { zulo) } \\
\end{array}$ & $\begin{array}{l}1 \text { ch. p } \\
\text { dora } 38 \\
100 \mathrm{cv} \text {. }\end{array}$ \\
\hline $\mathrm{TE}$ & $\begin{array}{l}1 \text { transformador elétrico } \\
\text { trifásico classe } 15 \mathrm{kV}, \\
150.000 \text { VA. }\end{array}$ & $\begin{array}{l}1 \text { transformador elétrico } \\
\text { trifásico classe } 15 \mathrm{kV} \text {, } \\
90.000 \mathrm{VA} \text {. } \\
\end{array}$ & $\begin{array}{l}\text { transformador elétrico } \\
\text { trifásico classe } 15 \mathrm{kV}, \\
89.100 \text { VA. }\end{array}$ & $\begin{array}{l}1 \text { transformador elétrico } \\
\text { trifásico classe } 15 \mathrm{kV} \text {, } \\
112.500 \text { VA. }\end{array}$ \\
\hline $\mathrm{AE}$ & $\begin{array}{lr}\text { acessórios } & \text { elétricos: } \\
\text { isoladores; } & \text { eletrodutos; } \\
\text { conectores; hastes de de } \\
\text { aterramento; cabos; etc. }\end{array}$ & $\begin{array}{|lr|}\text { acessórios } & \text { elétricos: } \\
\text { isoladores; } & \text { eletrodutos; } \\
\text { conectores; } & \text { hastes de } \\
\text { aterramento; } & \text { cabos; etc. }\end{array}$ & \begin{tabular}{lr|} 
acessórios & elétricos: \\
isoladores; & eletrodutos; \\
conectores; & hastes de \\
aterramento; & cabos; etc.
\end{tabular} & $\begin{array}{l}\text { acessórios elétricos: } \\
\text { isoladores; eletrodutos; } \\
\text { conectores; hastes de } \\
\text { aterramento; cabos; etc. }\end{array}$ \\
\hline LAT & $\begin{array}{l}1 \text { linha de alta tensão de } \\
1800 \mathrm{~m} \text {, padrão CPFL. }\end{array}$ & $\begin{array}{l}1 \text { linha de alta tensão de } \\
1800 \mathrm{~m} \text {, padrão CPFL. }\end{array}$ & $\begin{array}{l}\text { linha de alta tensão de } \\
1800 \mathrm{~m} \text {, padrão CPFL. } \\
\end{array}$ & $\begin{array}{l}1 \text { linha de alta tensão de } \\
1800 \mathrm{~m} \text {, padrão } \mathrm{CPFL} \text {. }\end{array}$ \\
\hline $\mathrm{OCC}$ & $\begin{array}{l}1 \text { casa de bombas de } \\
14,00 \mathrm{~m}^{2} \text {, acabamento } \\
\text { rústico. }\end{array}$ & $\begin{array}{l}\text { casa de bombas de } \\
14,00 \mathrm{~m}^{2}, \text { acabamento } \\
\text { rústico. }\end{array}$ & \begin{tabular}{|l|}
1 casa de bombas de \\
$14,00 \mathrm{~m}^{2}$, \\
rústico.
\end{tabular} & $\begin{array}{l}1 \text { casa de bombas de } \\
14,00 \mathrm{~m}^{2} \text {, acabamento } \\
\text { nústico. }\end{array}$ \\
\hline
\end{tabular}


APENDICE 11. Comportamento do custo anual total do sistema (CATS), custo anual de bombeamento (CABO), custo fixo anual do sistema (CFAS) e custo anual com manutenção e reparos (CAMR) em função do diâmetro da tubulação de recalque para os sistemas com bomba hidráulica acionada por motor a óleo diesel e à eletricidade nas diferentes modalidades.

\section{Sistema a óleo diesel}

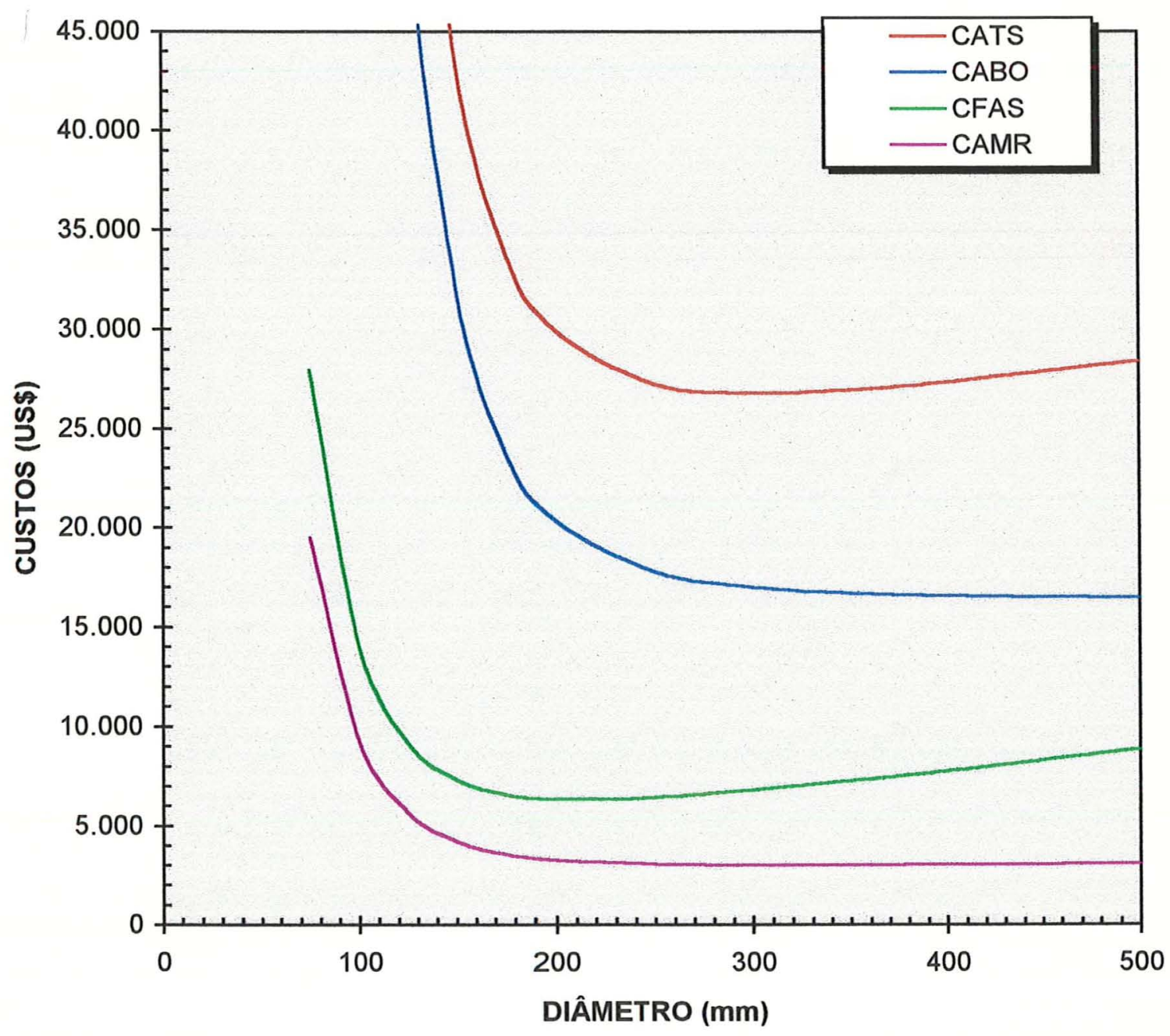


2. Sistema à eletricidade na tarifa convencional normal

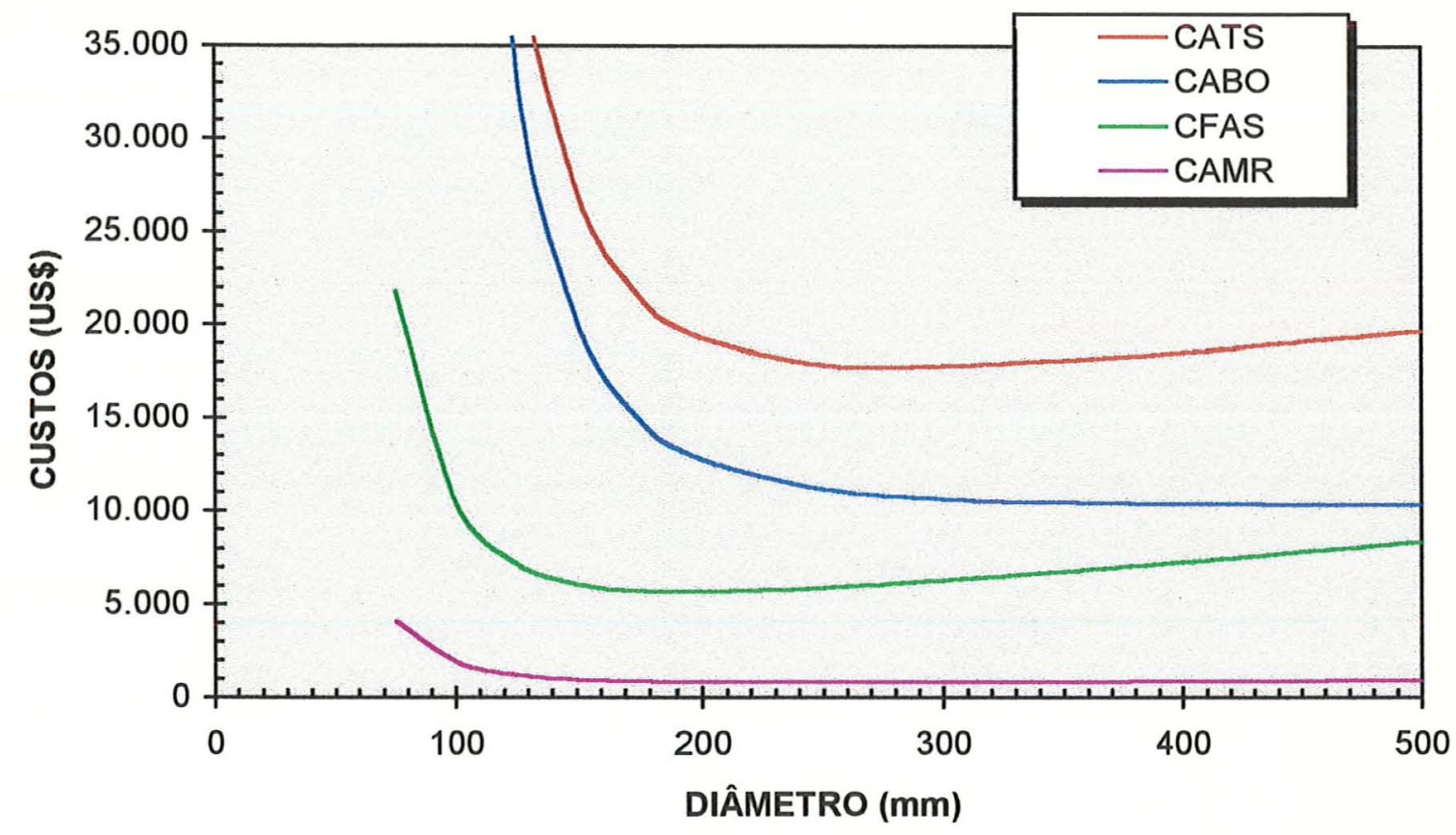

3. Sistema à eletricidade na tarifa convencional irrigante

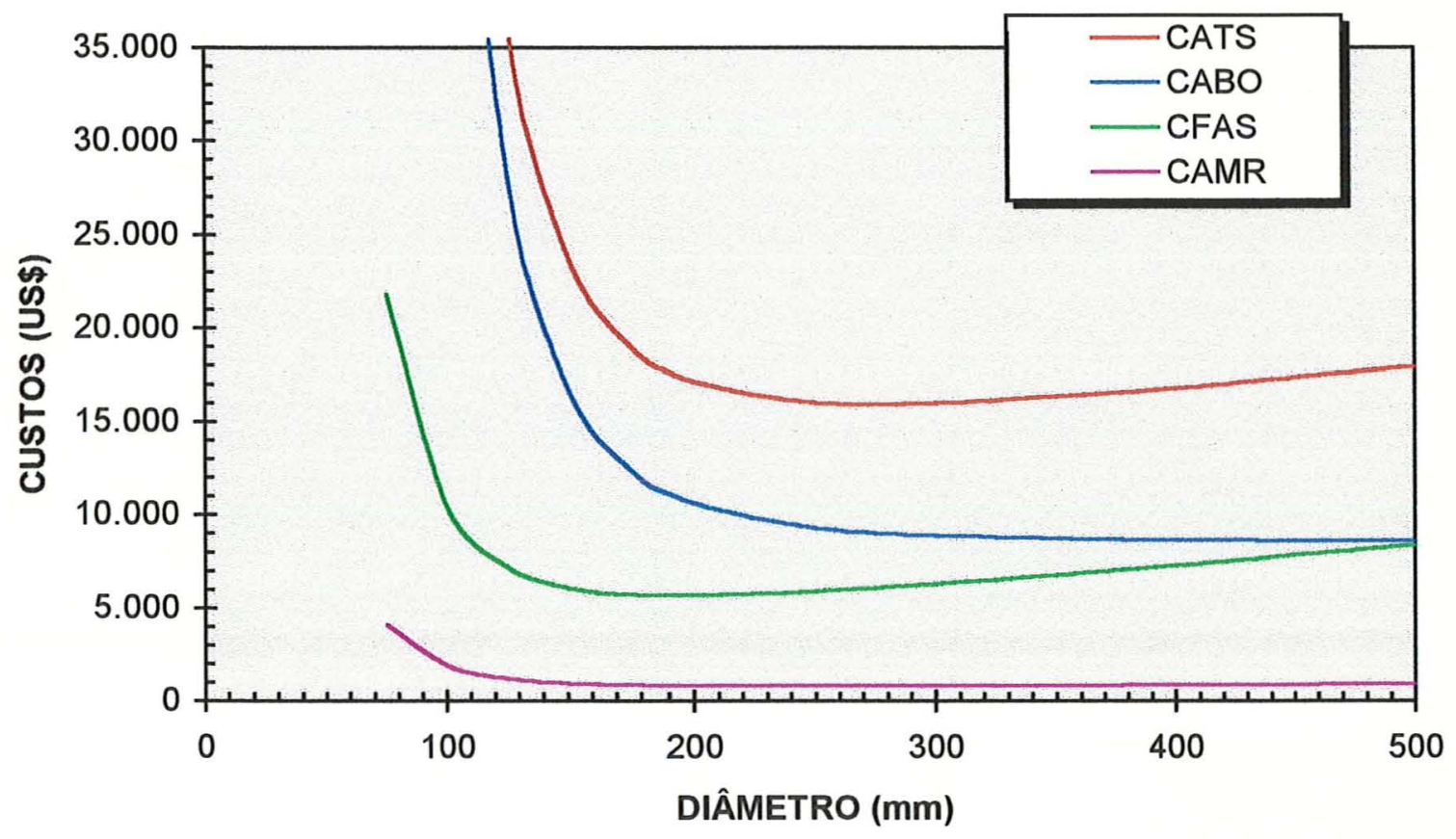


4. Sistema à eletricidade na tarifa verde normal

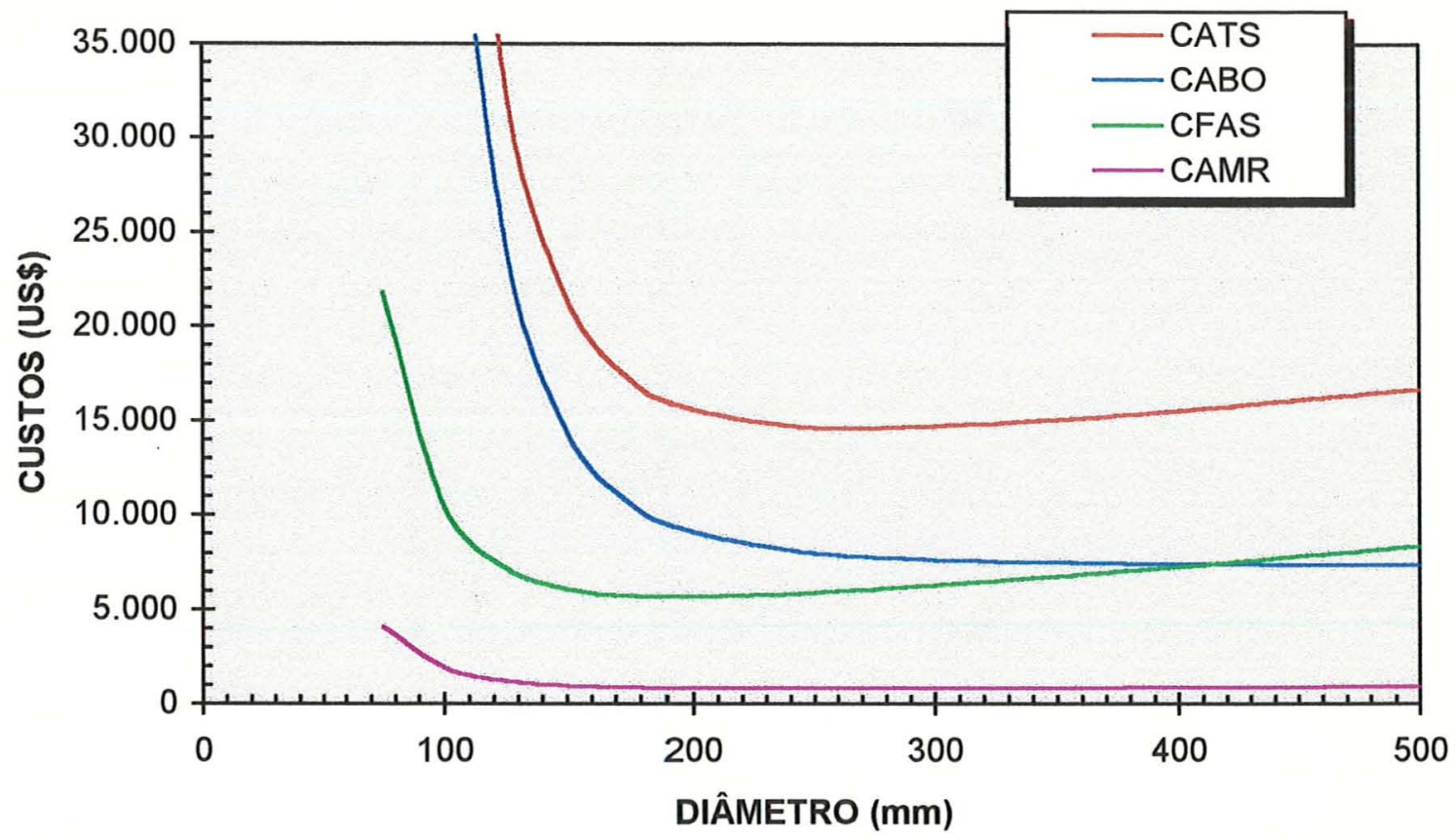

5. Sistema à eletricidade na tarifa verde irrigante

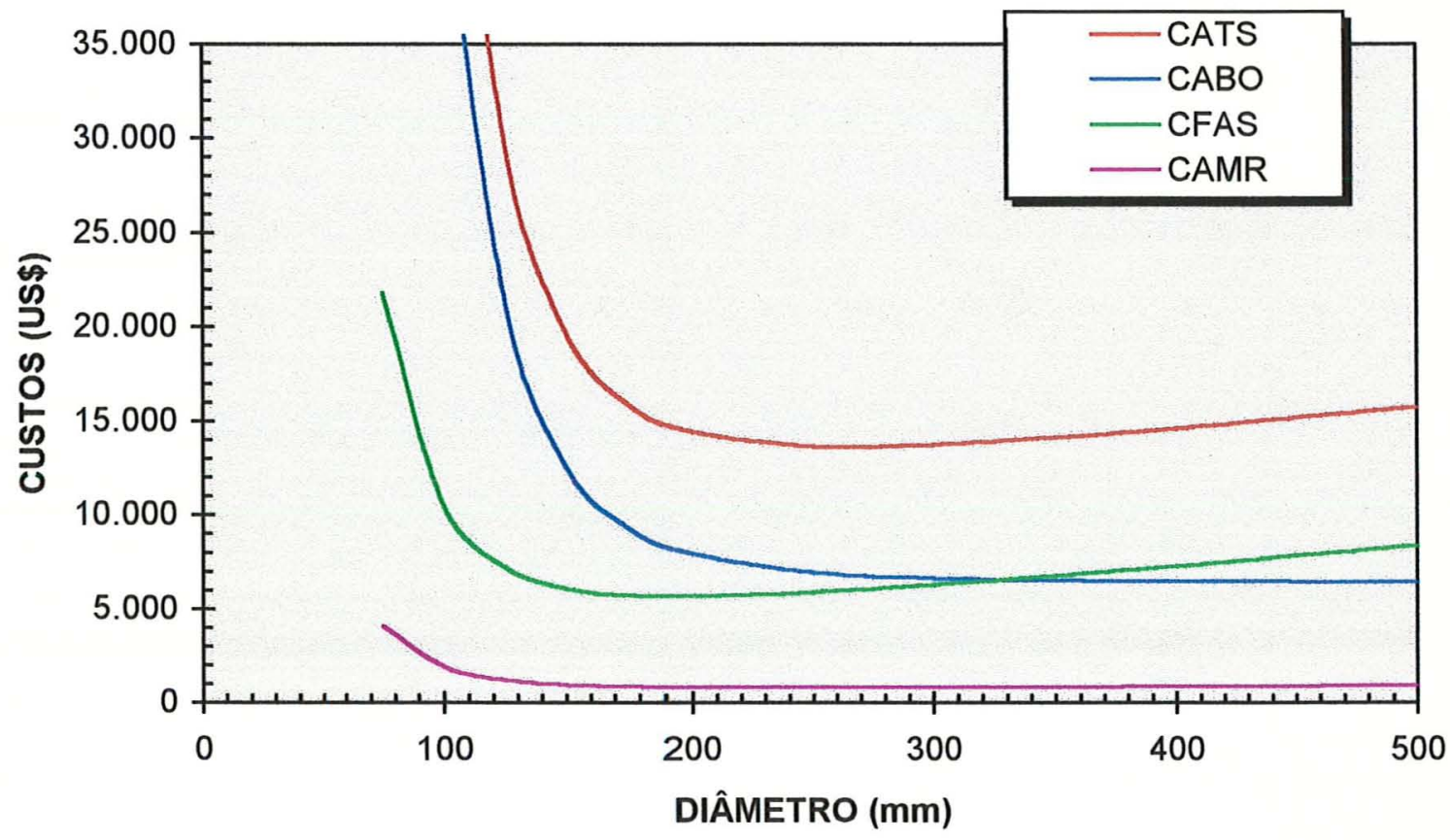


6. Sistema à eletricidade na tarifa azul normal

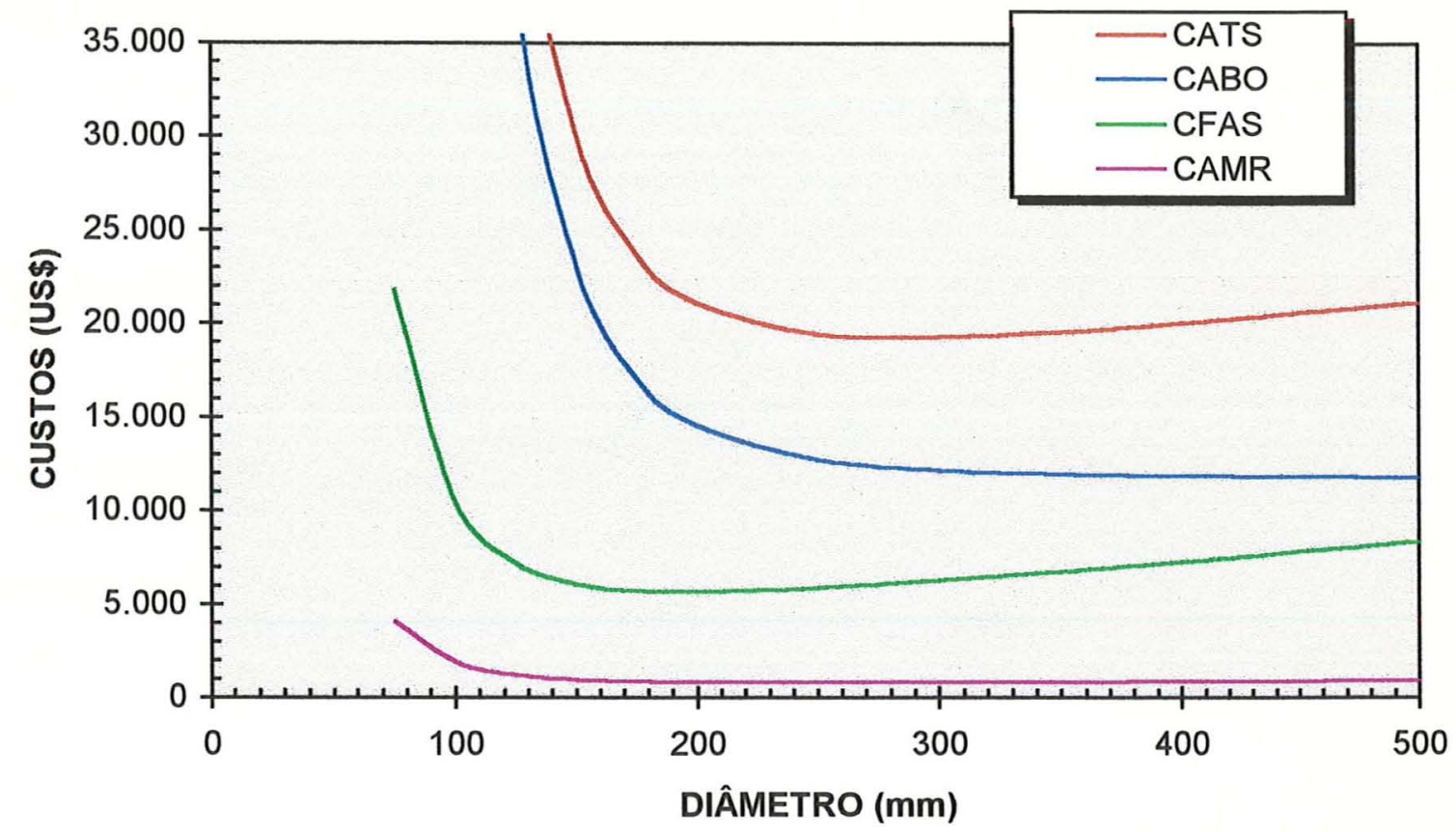

7. Sistema à eletricidade na tarifa azul irrigante

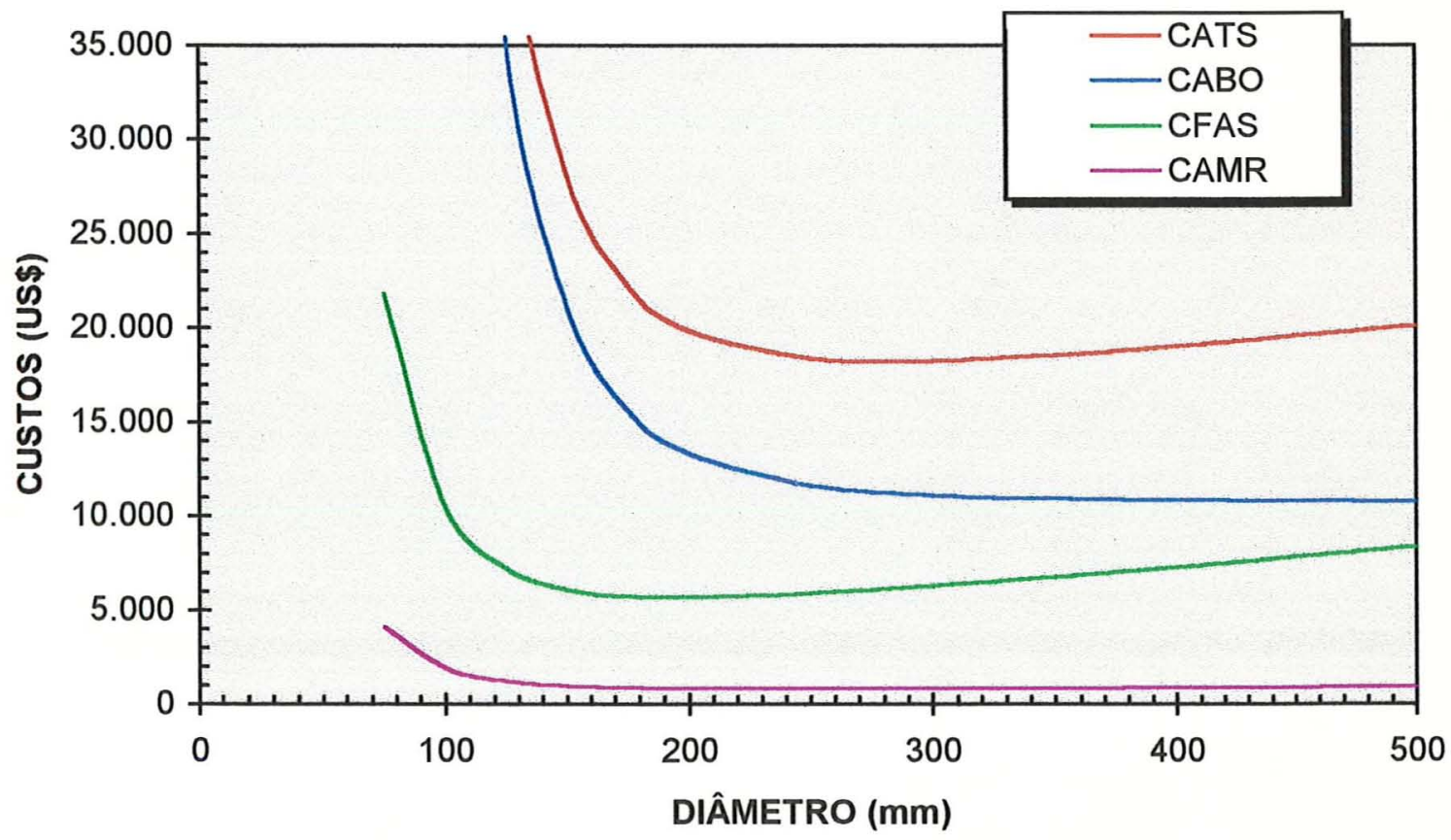

